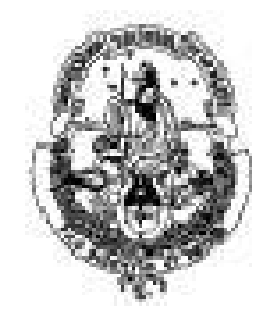

Facultad de Ciencias Astronómicas y Geofísicas Universidad Nacional de La Plata
Istituto Nazionale di Geofisica e Vulcanologia INGV, Roma

\title{
CARACTERIZACIÓN DE LA ESTRUCTURA DEL COMPLEJO VOLCÁNICO AUCA MAHUIDA MEDIANTE DATOS AEROMAGNÉTICOS Y GRAVIMÉTRICOS
}

\author{
Laura Mariana Longo
}

Tesis doctoral

Facultad de Ciencias Astronómicas y Geofísicas Universidad Nacional de La Plata

Junio 2017 
Caracterización de la estructura del complejo volcánico Auca Mahuida mediante datos aeromagnéticos y gravimétricos

Tesis presentada para optar por el grado de Doctor en Geofísica

Laura Mariana Longo

Director: Dr. Riccardo De Ritis

Istituto Nazionale di Geofisica e Vulcanologia di Roma, INGV, Italia

Co-directora: Dra. Claudia Tocho

Facultad de Ciencias Astronómicas y Geofísicas. Universidad Nacional de La Plata, Argentina

Facultad de Ciencias Astronómicas y Geofísicas

Universidad Nacional de La Plata

Junio 2017 
A mis dos amores, Clara y Esteban

A mis padres

A mi dulce Salvi. Te extraño. 


\section{AGRADECIMIENTOS}

Quiero expresar mi profundo agradecimiento a Esteban y Clarita quienes cedieron su tiempo para que pudiera estudiar, cursar materias, viajar a Italia, y formaron parte del proceso de esta tesis.

A Riccardo De Ritis con quien crecí profesionalmente en los temas de esta tesis y por la confianza que depositó en mí desde el momento en que decidí comenzar esta etapa bajo su dirección.

A mi mamá por su disponibilidad para cuidar de mi dulce Clara en los momentos que la necesité.

A YPF S. A. que cedió los datos para que pudiera desarrollar la tesis y especialmente al sector de Geofísica.

A Claudia Tocho quién codirigió mi carrera de doctorado y por su positivismo.

A Claudia Ravazzoli, quien me inició en Métodos Potenciales y también porque pude contar con ella en algunos temas de la tesis.

A Guido Ventura por su gran apoyo en el trabajo de campo y en los conceptos geológicos y estructurales del área de estudio.

A Telma Aisengart Santos por su guía en el manejo del modelado de inversión.

A Massimo Chiappini, por permitirme tener dos estadías en el Istituto Nazionale di Geofisica e Volcanologia, en la bella ciudad de Roma.

A Fabbio Speranza por las discusiones sobre cuestiones de paleomagnetismo.

A Augusto Rapallini quién me permitió y guió a realizar mediciones en muestras de roca en el laboratorio de Paleomagnetismo de la Universidad de Buenos Aires.

A Rubén Somoza por su colaboración para elegir las muestras de las coronas de pozos.

A Teresa Santana por sus sugerencias en la parte de sísmica.

A todos y cada uno de ustedes, simplemente GRACIAS... 


\section{RESUMEN}

El volcán Auca Mahuida es un centro eruptivo del Plioceno-Pleistoceno perteneciente a la provincia volcánica de retroarco de la Payenia, ubicada en el sector oriental de la Cuenca Neuquina en Argentina. El análisis de datos aeromagnéticos de alta resolución, integrado con la información geológica disponible y con mediciones de susceptibilidad magnética de rocas, ha permitido la construcción de un modelo geofísico confiable de la estructura interna del volcán. Se muestra en este trabajo que los datos aeromagnéticos constituyen una herramienta de investigación eficaz en un caso en el cual otros métodos, tales como la sísmica, no son aplicables debido a la topografía accidentada y a la alta impedancia acústica de la cubierta basáltica superior, cuyo espesor alcanza los 400 metros en el sector central del volcán. La mayor parte de las anomalías magnéticas presenta una estructura dipolar, opuesta a la que se esperaría para el campo geomagnético actual. De acuerdo con datos geocronológicos disponibles y con los resultados de mediciones paleomagnéticas, los cuerpos fuente del Auca Mahuida se emplazaron principalmente durante el cron Matuyama de polaridad reversa. La técnica de reducción al polo confirma que la dirección de la magnetización es principalmente reversa, con pocas anomalías magnetizadas en forma directa. Esta situación condujo a encontrar un enfoque adecuado de modelado inverso que considera la influencia de la remanencia y es independiente de la dirección de la magnetización, como resultado del cual se obtuvieron cuerpos fuente debajo del cráter central del Auca Mahuida a profundidades no mayores a $2 \mathrm{~km}$ bajo el nivel del mar. Estos resultados apoyan el concepto de un emplazamiento de los diques y cuerpos intrusivos en este rango de profundidades, controlado por las fallas que afectan el basamento y la secuencia sedimentaria sobre éste. El magma que ascendió a lo largo de esas fallas alimentó la actividad volcánica y luego se solidificó. Los resultados de los métodos de inversión empleados indican profundidades de fuentes consistentes con las de los cuerpos intrusivos observados en sondeos del área. Los pozos confirman la presencia de cuerpos ígneos intrusivos debajo del cráter central del Auca Mahuida y sectores periféricos, a profundidades que no exceden los $2 \mathrm{~km}$ bajo el nivel del mar. Por otro lado, las anomalías gravimétricas del volcán muestran un mínimo en el cráter central, interpretado como la respuesta de rocas traquíticas (cuya densidad y magnetización son menores que las correspondientes a los basaltos) y de los efectos del fracturamiento y colapso debidos a la actividad volcánica. Las profundidades de estas fuentes coinciden con las aportadas por el análisis de la magnetometría.

Los resultados obtenidos contribuyen a una mejor comprensión del sistema de alimentación del volcán y la geometría de los cuerpos fuente, demostrando la eficacia del modelado de anomalías magnéticas aún en presencia de fuerte magnetización remanente. 


\section{ABSTRACT}

The Auca Mahuida volcano is a Plio-Pleistocene eruptive center belonging to the Payenia retro-arc volcanic province, located in the eastern sector of the Neuquén basin, Argentina. The analysis of high-resolution aeromagnetic data, integrated with the available geological information and rock magnetic susceptibility measurements, allowed the construction of a reliable geophysical model of the inner structure of the volcano. Aeromagnetic data is shown to be an effective investigation tool where other methods, such as seismic, are not applicable due to the rough topography and the high acoustic impedance of the upper basaltic cover, whose thickness is about $400 \mathrm{~m}$ in the central sector of the volcano. Most of the magnetic anomalies have dipolar structure, opposite to that expected for the present geomagnetic field. According to the available geochronological data along with the results of paleomagnetic measurements, the source bodies of Auca Mahuida were emplaced mainly in the Matuyama reverse polarity chron. The Reduction-to-the-Pole technique confirms that the magnetization direction is mainly reverse with a few anomalies directly magnetized. This situation motivated an appropriate inverse model approach which considers the influence of the remanence and is independent of the magnetization direction. Such modelling identified bodies below the Auca Mahuida central crater at depths not larger than $2 \mathrm{~km}$ below sea level. These results indicate the emplacement of dikes and intrusive bodies at this depth range, controlled by the faults affecting the Auca Mahuida deeper basement and the above sedimentary sequence. Magma upraised along these faults feeding the volcanic activity and subsequently crystallized. The results of the applied inversion methods indicate sources depths consistent with those of the intrusions observed in wells. In fact, boreholes confirm the widespread presence of intrusive igneous bodies below the Auca Mahuida central crater and the peripheral sectors, at depths of about $2 \mathrm{~km}$ below sea level. On the other hand, gravity anomalies in the volcano show a minimum in the central crater area, interpreted as the response from trachitic rocks (whose density and magnetization are lower than those of basalts) and as the effect of fracturing and collapse of rocks in that area due to the volcanic activity. The depths of such sources coincide with those resulting from the magnetic anomaly analysis.

The obtained results shed light on the Auca Mahuida feeding system and on the sources geometry, demonstrating the effectiveness of the magnetic anomaly modeling even in presence of strong remanent magnetization. 


\section{ÍNDICE}

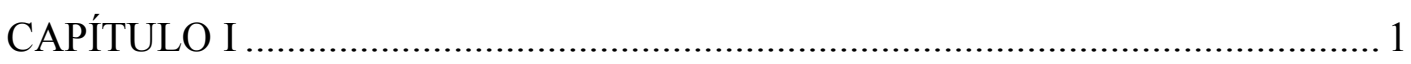

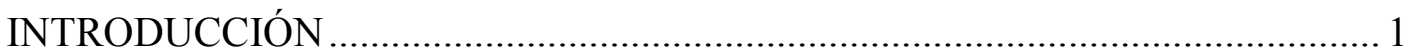

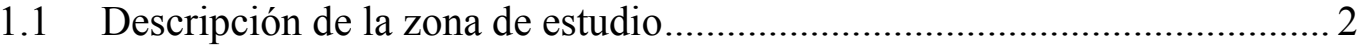

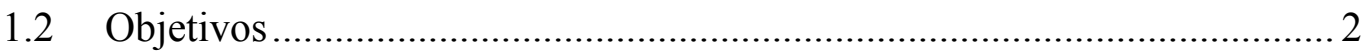

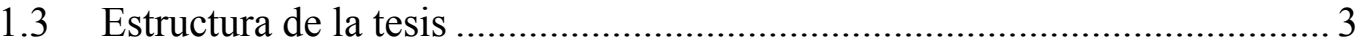

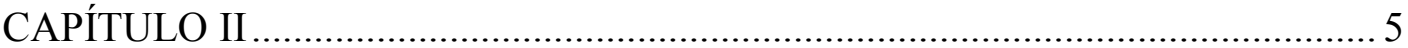

MARCO GEOLÓGICO DEL VOLCÁN AUCA MAHUIDA …............................. 5

2.1 Antecedentes de estudios de volcanes con métodos potenciales ................ 5

2.2 Marco geológico del Volcán Auca Mahuida …........................................... 7

2.3 Tipos de rocas y caracterización geoquímica …........................................ 11

2.4 Reservorios de hidrocarburos ................................................................... 12

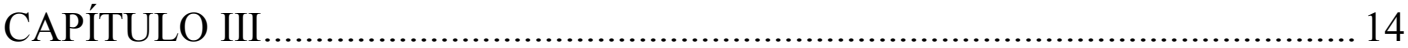

DESCRIPCIÓN Y TRATAMIENTO DE LOS DATOS ........................................ 14

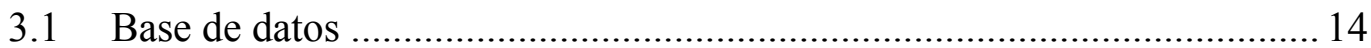

3.2 Adquisición y reducción de los datos magnéticos y gravimétricos en el

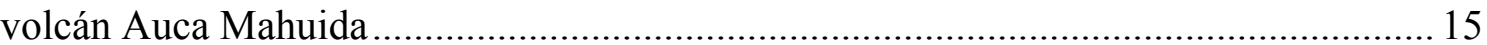

3.2.1 Generalidades sobre Campo geomagnético .................................... 17

3.2.2 Procesamiento del dato aeromagnético............................................ 20

3.2.3 Procesamiento del dato gravimétrico ............................................ 23

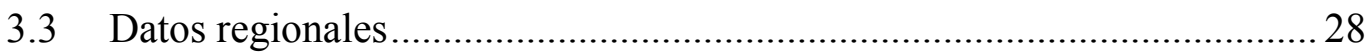

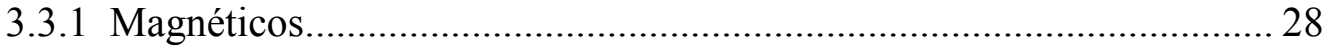

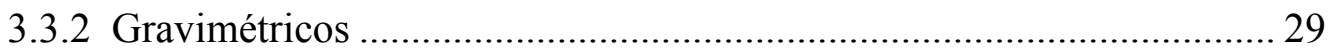

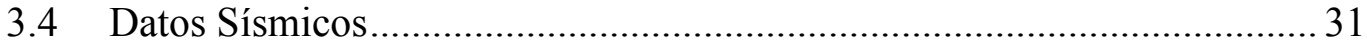

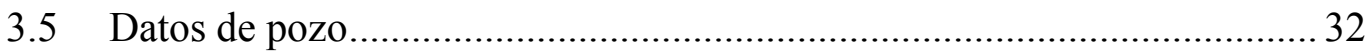

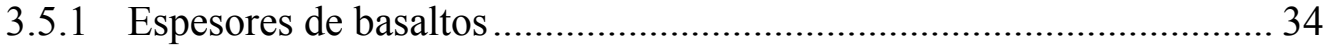


3.5.2 Temperatura del subsuelo y su relación con las propiedades magnéticas

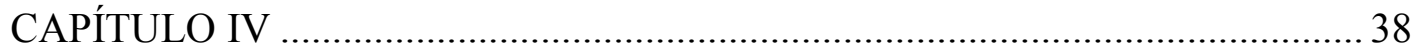

PROPIEDADES MAGNÉTICAS DE LAS ROCAS …...................................... 38

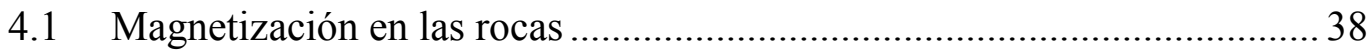

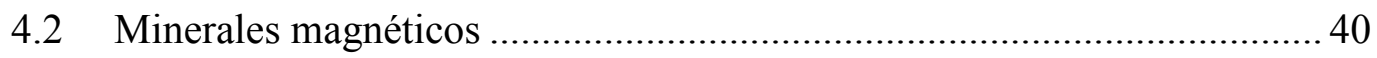

4.3 Muestreo paleomagnético, medición de la NRM y desmagnetización...... 41

4.4 Muestras obtenidas en el complejo volcánico Auca Mahuida................... 43

4.5 Muestras de corona del pozo VAM-28 ….......................................... 47

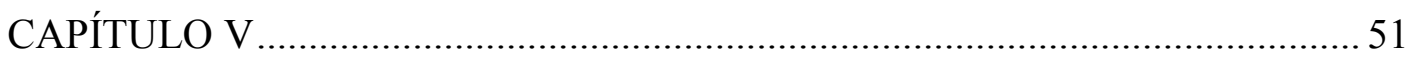

ANÁLISIS E INTERPRETACIÓN DE LAS ANOMALÍAS MAGNÉTICAS ..... 51

5.1 Campo de anomalías magnéticas ....................................................... 51

5.2 Análisis espectral del campo de anomalías magnéticas.............................53

5.3 Técnicas de análisis de las anomalías magnéticas ................................... 55

5.3.1 Efecto topográfico de una placa uniformemente magnetizada ............56

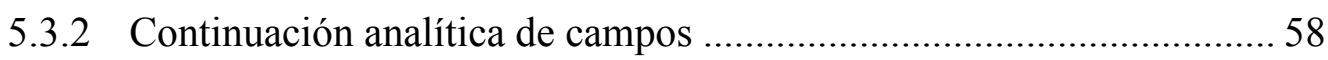

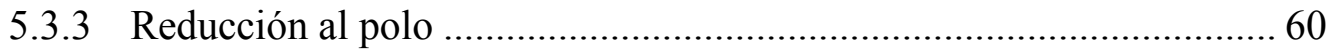

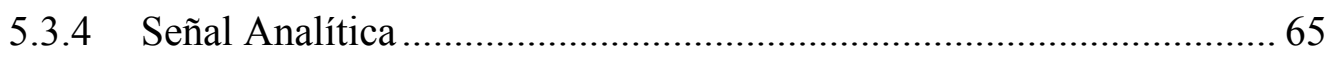

5.3.5 Métodos de localización y profundidad de fuentes magnéticas........... 68

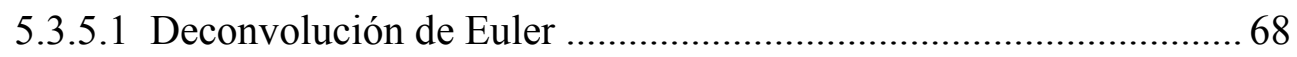

5.3.5.2 Profundidad media de un conjunto de fuentes............................... 74

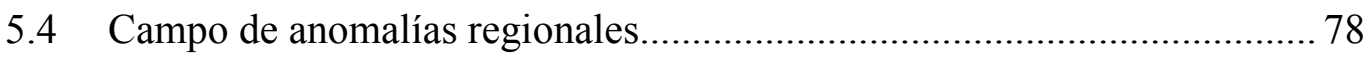

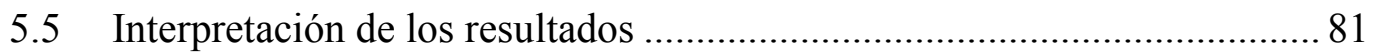

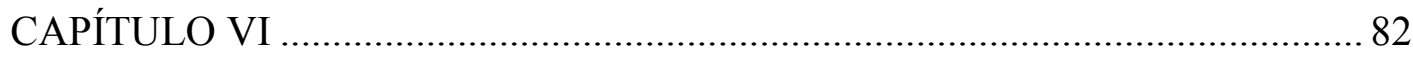

INTERPRETACIÓN DE LOS DATOS GRAVIMÉTRICOS Y SÍSMICOS ......... 82

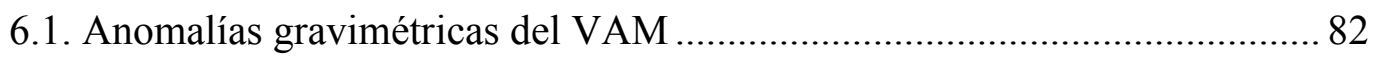

6.1.1 Determinación del valor de densidad topográfica ….......................... 85

6.1.2 Espectro de potencia radial promediado ......................................... 90

6.1.3 Filtrado del campo de anomalías gravimétricas................................. 91

6.2 Datos gravimétricos terrestres regionales ............................................... 98 
6.3 Conceptos básicos de sísmica de reflexión.............................................. 101

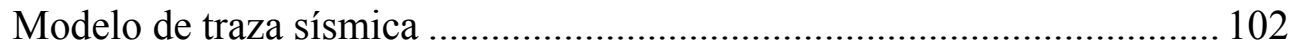

6.4 Interpretación del dato sísmico ........................................................... 103

6.4.1 Calibración de sísmica con perfiles de pozos .................................. 104

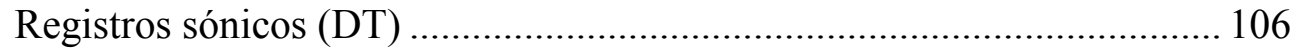

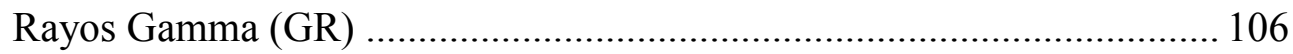

6.4.2 Interpretación de horizontes y fallas ............................................. 107

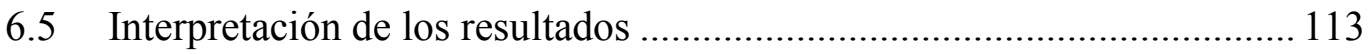

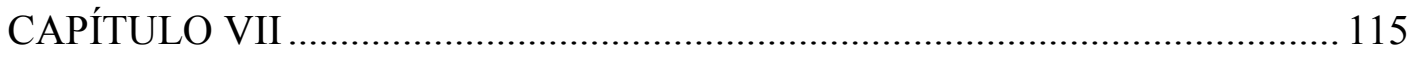

MODELADO MAGNÉTICO Y GRAVIMÉTRICO …....................................... 115

7.1 Modelado de las anomalías ................................................................ 115

7.2 Formulación del problema inverso- Caso magnético ............................. 116

7.2.1 Aplicación a los datos magnéticos del VAM ............................... 118

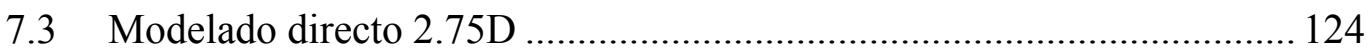

7.3.1 Modelado magnético de las anomalías del VAM ........................ 124

7.3.2 Modelado 2.75 D gravi-magnetométrico de los datos del VAM.. 131

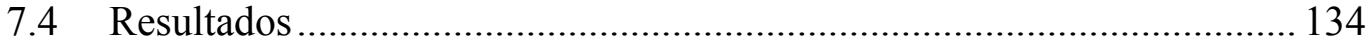

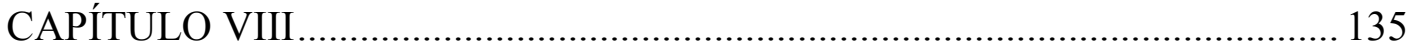

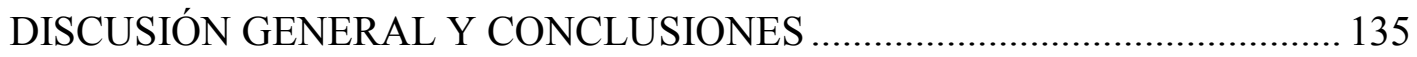

8.1 Discusión sobre los resultados ............................................................. 135

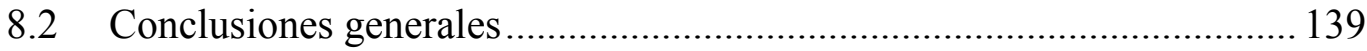

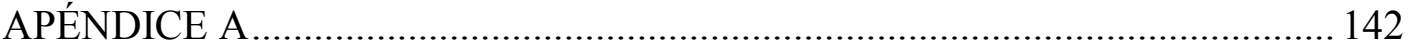

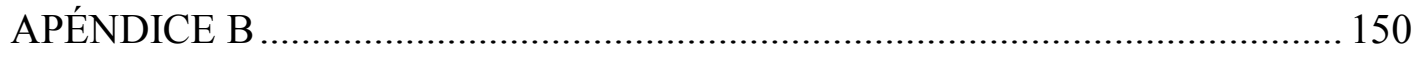

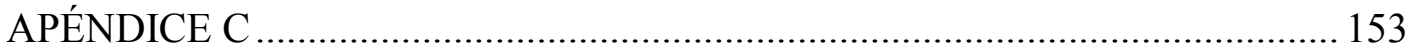

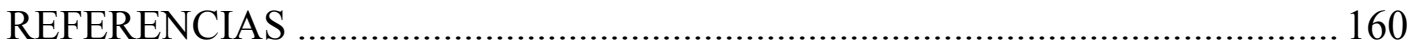




\section{LISTA DE FIGURAS}

Figura 2.1. Arriba izquierda: ubicación de la Cuenca Neuquina (contorno rojo sobre el mapa de Argentina) junto con el plateau Auca Mahuida dentro de la cuenca. Arriba derecha: mapa esquemático de la provincia volcánica de Payenia. Abajo: imagen satelital del complejo volcánico con indicación de los principales rasgos estructurales.

Figura 2.2. Columna estratigráfica correspondiente al área del volcán Auca Mahuida (Brisson \& Veiga, 1999).

Figura 2.3. Modelo esquemático propuesto para el origen de las lavas de retroarco dentro de la provincia volcánica de Payenia. Se muestra la placa oceánica deformada y la consecuente surgencia de material astenosférico. Figura tomada de Pallares et al. (2016).

Figura 2.4. Imagen Satelital IKONOS, del año 2002, resolución $1 \mathrm{~m}$. En el óvalo amarillo se muestra la localización del yacimiento Volcán Auca Mahuida.

Figura 3.1. Mapa del modelo de elevación digital del terreno del Volcán Auca Mahuida provisto por YPF S A. Las unidades están dadas en m sobre nivel del mar. Resolución: $90 \mathrm{~m}$ x $90 \mathrm{~m}$. En círculo blanco se indica el cráter central.

Figura 3.2. Patrón de líneas de vuelo del relevamiento aeromagnético-gravimétrico 2001 en color negro. Contorno: plateau volcánico. Se puede apreciar que el relevamiento aeromagnético no cubre todo el plateau.

Figura 3.3. Gráfico adaptado de Blakely, 1995. a) Sistema de coordenadas esféricas. El punto $\mathrm{P}$ está definido por las coordenadas $\mathrm{r}, \theta$ y $\emptyset$, y un vector en el punto $\mathrm{P}$ se describe en términos de tres vectores unitarios ortogonales: $\hat{r}, \widehat{\Theta}, \mathrm{y} \widehat{\Phi}$. b) Sistema de coordenadas cartesianas del punto $P$. Se muestran las tres componentes del vector $B$ : $B_{\mathrm{x}}$ es positivo en dirección norte $\left(B_{\mathrm{x}}=-B_{\theta}\right), B_{\mathrm{y}}$ positivo hacia el este $\left(B_{\mathrm{y}}=B_{\varnothing}\right)$, y $\mathrm{B}_{\mathrm{z}}$ es positivo hacia abajo $\left(B_{\mathrm{z}}=-B_{\mathrm{r}}\right)$. La inclinación $I$ es el ángulo que forma $B$ con el plano horizontal, positivo hacia abajo; la declinación $D$ es el acimut de la proyección horizontal de $B$, positivo hacia el este.

Figura 3.4: Corrección de Eötvös como función del rumbo con los parámetros promedio del relevamiento.

Figura 3.5. Datos aeromagnéticos regionales. El patrón de líneas de vuelo se representa en puntos de color azul. Se observa que los datos no cubren completamente el plateau volcánico del volcán Auca Mahuida.

Figura 3.6. Datos gravimétricos regionales adquiridos por YPF.

Figura 3.7. Líneas sísmicas 2D reprocesadas (color amarillo) sobre imagen satelital. El rectángulo blanco indica la ausencia de relevamiento sísmico 3D.

Figura 3.8. Unión de los tres relevamientos reprocesados para formar el cubo El 
Volcán 3D (polígono celeste).

Figura 3.9. Ubicación de los pozos analizados que atraviesan cuerpos intrusivos.

Figura 3.10. Espesores en metros de roca ígnea de la cubierta lávica atravesada por pozos.

Figura 3.11. Temperatura versus profundidad. a) Los círculos marrones represenan los datos de temperatura de pozo de la zona del Auca Mahuida. En rojo se muestra la recta de tendencia global promedio de la cuenca. b) Los triángulos son los datos de temperatura de los 17 pozos. Las rectas representan a las distintas tendencias geotérmicas zonales dentro de la cuenca (Sigismondi, 2012).

Figura 4.1. Magnetización resultante de la suma de las componentes de magnetización inducida, $M \mathrm{i}$, y remanente, $M \mathrm{r}$.

Figura 4.2. Diagrama ternario para los óxidos de hierro, modificado de Lowrie (2007). Las líneas continuas son las series de solución.

Figura 4.3. Foto de la muestra AM15 con su orientación, tomada durante campaña.

Figura 4.4. Imagen de la distribución de muestras en el campo y medición de la susceptibilidad magnética in situ, puntos rojos sobre plateau.

Figura 4.5. Modificado de Butler, 1992. Izquierda: Escala de Cox (1982) de los primeros 60 M.a. Derecha: Escala de Mankinen \& Dalrymple (1979) basada en determinaciones de edad por K-Ar, polaridad paleomagnética e información complementaria de perfiles marinos de anomalías magnéticas y de datos paleomagnéticos de coronas de lecho marino profundo. Cada línea horizontal en las columnas indicadas como polaridad normal, polaridad intermedia o polaridad reversa representa una roca ígnea Las flechas indican intervalos cortos de polaridad llamados "excursiones" geomagnéticas; los números a la derecha de la columna de polaridad indican las edades interpretadas de los límites entre polaridades distintas.

Figura 4.6. Ubicación del pozo VAM-28 (círculo naranja en la imagen satelital).

Figura 4.7. Fragmentos de diques basálticos atravesados por el pozo.

Figura 4.8: Muestras cortadas listas para medir.

Figura 4.9. Magnetómetro JR-6 AGICO Dual Speed Spinner magnetometer.

Figura 4.10. Desmagnetizador por campos alternos, AF Demagnetizer LDA.

Figura 5.1. Mapa de anomalías magnéticas TMI correspondiente al relevamiento aeromagnético 2001, superpuesto a imagen satelital.

Figura 5.2. Efecto topográfico producido por una placa cortical uniformemente magnetizada de $2 \mathrm{~A} / \mathrm{m}$ en un campo de polaridad reversa $\left(I=35^{\circ}, D=190^{\circ}\right)$.

Figura 5.3. Anomalía magnética sintética (original y reducida al polo) producida por una línea de dipolos, ubicada en $\mathrm{x}_{0}=40, \mathrm{z}_{0}=10 \mathrm{~km}$. El campo ambiente es de $26000 \mathrm{nT}$ y la susceptibilidad magnética de 0.012 (SI). La declinación e inclinación magnética 
son $0^{\circ}$ y $-30^{\circ}$, respectivamente y el acimut $=0^{\circ}$. El eje horizontal representa la posición en la dirección perpendicular a la línea de dipolos y el eje vertical corresponde a la magnitud de las anomalías, dado en nT.

Figura 5.4. Mapa de anomalía magnética del volcán Auca Mahuida. El círculo blanco marca el cráter central. Límite del plateau volcánico es indicado en línea negra. Las letras I, J, K, L, y M especifican las anomalías discutidas en el texto. El relevamiento aeromagnético no cubre todo el plateau.

Figura 5.5. a) Transformación RTP calculada considerando solamente la componente de inducción $\left(I=-38^{\circ}\right.$ and $\left.D=3^{\circ}\right)$. b) Transformación RPT calculada con ambas componentes de magnetización, de inducción y remanente $\left(I=38^{\circ}\right.$ and $\left.D=177^{\circ}\right)$. Las anomalías están etiquetadas con letras en color blanco y negro respectivamente (ver Figura 5.4 para su comparación).

Figura 5.6. Mapa de amplitud de la señal analítica obtenido de la anomalía TMI prolongada hacia arriba $50 \mathrm{~m}$. El círculo de línea discontinua señala el cráter central del volcán. S1 a S10 son las fuentes principales causantes de los máximos de amplitud. La unidad de la amplitud de la SA es [nT/m].

Figura 5.7. Soluciones de deconvolución de Euler obtenidas a partir del mapa de anomalías magnéticas con un tamaño de ventana de $5 \mathrm{~km}$ para un índice estructural dado. a) $N=1$ y b) $N=0.5$. La elevación de referencia es el nivel medio del mar dado en metros. En la figura a) I, J, K, M, L son las letras utilizadas en las anomalías de la RTP de la figura 5.5. b) Máximos de amplitud producidos por las principales fuentes en la señal analítica de la figura 5.7 a (S1, S2, S3, S4, S5, S6, S7, S8, S9, S10). En trazos amarillos se superpone el patrón de fallas interpretadas en superficie por Del Pino (YPF S. A.).

Figura 5.8. Soluciones de Euler obtenidas a partir del mapa de anomalías magnéticas con un tamaño de ventana de $3 \mathrm{~km}$ para un índice estructural $N=1$.

Figura 5.9. Soluciones de Euler obtenidas a partir del mapa de anomalías magnéticas con un tamaño de ventana de $10 \mathrm{~km}$ para un índice estructural $N=1$. a) Tamaño de celda de la grilla $500 \mathrm{~m}$ y b) $1000 \mathrm{~m}$.

Figura 5.10. a) Profundidad de las fuentes magnéticas estimada a partir del espectro de potencia logarítmico promediado radialmente del mapa de anomalías. El espectro corresponde a grupos de cuerpos magnéticos con la misma profundidad media. $\mathrm{k}^{*}$ es el número de onda correspondiente al máximo en el espectro de potencia. b) Profundidades estimadas a partir de la pendiente del espectro tomada en intervalos de 5 puntos.

Figura 5.11. a) Relevamiento regional de 1968. R1, R2, R3 y R4 representan a las anomalías de longitudes de onda larga. En línea negra se indica el límite del plateau volcánico y las líneas de vuelo de la adquisición de los datos locales se grafican sobre la grilla. b) Campo de anomalías del relevamiento 2001. L1, L2, L3, L4, L5, L6 y L7 representan a las anomalías individuales principales. La escala TMI está dada en [nT] para ambas figuras.

Figura 5.12. Principales rasgos estruturales del complejo volcánico Auca Mahuida. R1, R2, R3 y R4 correspenden a las anomalías mostradas en los datos aeromagnéticos de la figura $5.11 \mathrm{~b}$ ). 
Figura 6.1. Mapa de anomalía de aire libre obtenido por Carson.

Figura 6.2. Curvas con valores de anomalía de aire libre (naranja) y topografía

(celeste) sobre el perfil en línea negra NO-SE graficado sobre el mapa de anomalía.

Figura 6.3. Anomalía de Bouguer obtenida por la empresa Carson Aerogravity con una 85 densidad de $2.43 \mathrm{~g} / \mathrm{cm}^{3}$.

Figura 6.4. Anomalía de Bouguer simple calculada con una densidad de $2.43 \mathrm{~g} / \mathrm{cm}^{3}$.

Figura 6.5. Anomalía de Bouguer completa (con corrección topográfica) calculada con una densidad de $2.43 \mathrm{~g} / \mathrm{cm}^{3}$.

Figura 6.6. Profundidad de las fuentes gravimétricas estimada a partir del espectro de energía logarítmico promediado radialmente del mapa de anomalías de Bouguer completa. El espectro corresponde a grupos de cuerpos con la misma profundidad media.

Figura 6.7. a y c) Anomalía regional con ajuste polinómico de grado 1 y 3 , respectivamente. b y d) Anomalía residual resultado de la remoción de la anomalía regional en a) y c) respectivamente, a la anomalía de Bouguer completa.

Figura 6.8. Perfil SO-NE sobre el cual se grafica la respuesta de la ABC y sus componentes regionales y residuales (figuras 6.9, 6.10 y 6.12) obtenidas con los métodos mecionados en el texto.

Figura 6.9. Anonalía de Bouguer $\mathrm{ABC}$, junto a las componentes residual y regional resultado de aplicar una remoción de tendencia lineal sobre el perfil indicado en el mapa. Curva roja: $\mathrm{ABC}$, verde: componente residual y celeste: componente regional.

Figura 6.10. Anonalía de Bouguer ABC, junto a las componentes residual y regional resultado de aplicar una remoción de tendencia de polinomio de grado 3 sobre el perfil indicado en el mapa. Curva azul: $A B C$, roja: componente residual y verde: componente regional.

Figura 6.11. a y c) Componente regional resultado de la prolongación ascendente de la anomalía de Bouguer completa a 10 y $20 \mathrm{~km}$, respectivamente. b y d) Anomalía residual de Bouguer resultado de la diferencia entre la anomalía de Bouguer completa y la componente regional a 10 y $20 \mathrm{~km}$, respectivamente.

Figura 6.12. Anonalía de Bouguer $\mathrm{ABC}$, junto a las componentes residual y regional resultado de la continuación ascendente a 10 y $20 \mathrm{~km}$. Curva verde: $\mathrm{ABC}$, gris y azul: continuación ascendente a 10 y $20 \mathrm{~km}$, respectivamente, rosa y roja: componente residual resultado de la continuación a 10 y $20 \mathrm{~km}$, respectivamente.

Figura 6.13. Filtro de Butterworth aplicado a los datos de anomalía de Bouguer completa. Se usó un número de onda de corte de $0.09 \mathrm{~km}^{-1}$, o sea una longitud de onda de $11 \mathrm{~km}$ para obtener la componente regional.

Figura 6.14. Gravedad observada. Los puntos en color morado corresponden a los 99 datos de gravedad terrestres medidos en la cuenca Neuquina. 
Figura 6.15. Anomalía de aire libre de los datos regionales terrestres.

Figura 6.16. Anomalía de Bouguer simple de los datos regionales terrestres obtenida 100 con una densidad de reducción de $2.67 \mathrm{~g} / \mathrm{cm}^{3}$. El VAM está ubicado entre un mínimo (norte) y máximo (sur) relativo. R1, R2, R3 y R4 representan las anomalías magnéticas identificadas en la figura 5.11.

Figura 6.17. Anomalía de Bouguer completa de los datos regionales terrestres 101 obtenida con una densidad de reducción de $2.67 \mathrm{~g} / \mathrm{cm}^{3}$. La corrección topográfica se realizó con el modelo digital del terreno de la figura 3.1.

Figura 6.18. Línea sísmica 2D yam-9723 NO-SE reprocesada en 2012 representada en color negro.

Figura 6.19. Sismograma sintético del pozo VAM-x.1. De izquierda a derecha: ley de velocidad obtenida del sismograma, perfil sónico (rojo), densidad (azul), rayos Gamma (verde); escala de tiempo y profundidad, pases formacionales, porción de la sección sísmica alrededor del pozo a izquierda y derecha y en el centro el sismograma sintético. Serie de coeficientes de reflexión en color rojo y ondícula.

Figura 6.20. Registros de rayos gamma (GR) medidos en grados API, perfil sónico (DT) en $\mu \mathrm{s} / \mathrm{ft}$ y densidad (RHOB) en $\mathrm{g} / \mathrm{cm}^{3}$ en los pozos VAM.x-1 y LRb.x-1. Los intervalos sombreados corresponden a los cuerpos de roca ígnea.

Figura 6.21. Corte sísmico indicado en línea roja en el mapa (margen inferior izquierdo) sobre cubo El volcán 3D.

Figura 6.22. Corte sísmico en el cubo de varianza sobre la línea roja del mapa de la figura 6.21 .

Figura 6.23. Vistas 3D de los principales horizontes sísmicos y dos filones capa, Sill QV1 y Sill QV2, en la formación Vaca Muerta en el cubo de amplitudes (cantidad sin dimensión).

Figura 6.24. Mapa isócrono (en tiempo) resultado de la interpretación sísmica del tope y base del filón Sill QV1.

Figura 6.25. Profundidades de los cuerpos intrusivos en el pozo VAM.x.1. Gráfico modificado de Bermúdez y Delpino (2002): descripción litológica a partir del cutting. La cota del pozo está expresada en metros sobre el nivel del mar [msnm].

Figura 7.1. Esquema del proceso de inversión.

Figura 7.2. Diagrama con las componentes obtenidas en el modelo inverso MVI.

Figura 7.3. Figura 7.3. Volumen de magnetización obtenido en el modelo de inversión MVI.

Figura 7.4. Diferencia entre los datos observados y el resultado del modelo predictivo.

Figura 7.5. Histograma de amplitudes de la magnetización obtenida del MVI. 
Figura 7.6. Isosuperficie de 0.012 SI que representa los cuerpos fuente de anomalía magnética (color celeste) sobre la topografía a); b) la misma isosuperficie que en a) sobre el mapa de señal analítica. c) Geometría de la isosuperficie de 0.012 SI en el cráter central del volcán. La superficie en el tope es la topografía. La dirección de $z$ es positiva arriba del nivel medio del mar.

Figura 7.7. Amplitud del MVI y señal analítica en planta.

Figura 7.8. Componente proyectada Eproy y perpendicular Eperp de la inversión MVI.

Figura 7.9. Perfiles $A B, C D$ y EF sobre el mapa de anomalías reducido al polo con los efectos de magnetización inducida y remanente, para realizar modelado.

Figura 7.10: Perfiles para realizar modelado sobre el mapa de anomalías reducido al polo con los efectos de magnetización inducida y remanente.

Figura 7.11: Convención de signos para modelado. Gráfico extraído de Geosoft.

Figura 7.12. Modelo 2.75D sobre perfil AB representado en el mapa de la figura 7.9. De arriba hacia abajo: vista en planta de los cuerpos, cuya extensión lateral está expresada en $\mathrm{km}$. Anomalía observada, calculada y curva de error (rojo). Modelado tomando en cuenta el modelo de inversión 3D (imagen superpuesta a los cuerpos) de la figura 7.10. A diferencia del modelado 3D, la dirección de $z$ es positiva debajo del nivel medio del mar.

Figura 7.13. Modelo 2.75D sobre perfil CD representado en el mapa de la figura 7.9.

Figura 7.14. Modelo 2.75D sobre perfil EF representado en el mapa de la figura 7.9.

Figura 7.15 Sección NO-SE sobre línea sísmica YAM-9723 para modelado 2.75D (línea violeta). Los círculos blancos representan los puntos de explosión de las líneas sísmicas 2D reprocesadas en el área. El perfil gravi-magnetométrico es más extenso de lo que lo es la línea sísmica.

Figura 7.16 Modelo gravi-magnetométrico sobre perfil NO-SE de la figura 7.15. Proyección del pozo VAM.x-1 sobre el modelo. Cota del pozo: $1676 \mathrm{~m}$ sobre nivel medio del mar, profundidad final del pozo $3486 \mathrm{~m}$. Debajo: Imagen de la línea sísmica YAM-9723 sobre la cual se ralizó el modelo.

Figura B1. Geometría de la capa magnética.

Figura C1. Prisma bidimensional $K L M N$ que se extiende al infinito en el eje $x$ positivo.

Figura C2. Construcción geométrica usada para calcular la anomalía causada por un cuerpo de sección poligonal KNPQRK.

Figura C3. Elementos que definen a las componentes del vector de magnetización. 


\section{LISTA DE TABLAS}

Tabla 2.1. Estructuras asociadas con el origen de anomalías magnéticas y gravimétricas en algunos volcanes previamente estudiados.

Tabla 3.1. Pozos que atraviesan cuerpos volcánicos.

Tabla 3.2. Espesores de basalto extraídos de los legajos de pozo. X e Y son las coordenadas planas Gauss Kruger faja 2 en el sistema geodésico Chos Malal, Z las cotas de superficie y la última columna los espesores de los basaltos de superficie para cada pozo.

Tabla 3.3. Datos de temperatura obtenidos de los legajos de pozo.

Tabla 4.1. Mediciones de las propiedades magnéticas de las rocas en laboratorio e in situ. En color negro se representa la polaridad normal, en blanco, la reversa. NA indica que la polaridad no pudo ser determinada.

Tabla 4.2. Valores de las mediciones de NRM y susceptibilidad magnética.

Tabla 6.1. Valores de densidad promedio para cada pozo obtenidos de los perfiles expresado en $\mathrm{g} / \mathrm{cm}^{3}$.

Tabla 6.1. Continuación. Valores de densidad promedio para cada pozo obtenidos de los perfiles expresado en $\mathrm{g} / \mathrm{cm}^{3}$.

Tabla 6.2. Rangos de valores de las distintas anomalías expresados en [mGal].

Tabla 6.3. Profundidades de los principales horizontes sísmicos en el pozo VAM.x-1.

Tabla 6.4. Clasificación de rocas volcánicas según su respuesta en los registros de pozo. Adaptada de Wohletz \& Heiken, 1992.

Tabla 7.1. Parámetros utilizados en el modelo del perfil AB.

Tabla 7.2. Parámetros utilizados en el modelo del perfil CD.

Tabla 7.3. Parámetros utilizados en el modelo del perfil EF.

Tabla 7.4. Parámetros utilizados en el modelo del perfil gravi-magnetométrico.

Tabla 8.1. Correlación entre fuentes magnéticas obtenidas con los métodos de RTP con inducción y remanencia (RTP_i+r), señal analítica (SA), y las anomalías 
modeladas con GMSYS (2.75D).

Tabla 8.2. Profundidad de los cuerpos magnéticos obtenidos a partir de la aplicación 141 de las técnicas utilizadas en este capítulo. La profundidad está dada en kilómetros respecto del nivel medio del mar (nmm), positivo hacia arriba y negativo debajo. 


\section{CAPÍTULO I}

\section{INTRODUCCIÓN}

El estudio de las anomalías magnéticas constituye una herramienta de gran utilidad para investigar la estructura interna de las áreas volcánicas debido a la alta respuesta magnética de las rocas ígneas. Las rocas volcánicas presentan magnetizaciones remanentes muy elevadas, superiores en varios órdenes de magnitud a las rocas sedimentarias. De este modo, las anomalías magnéticas nos permiten inferir la presencia de cuerpos ígneos no relacionados con la columna sedimentaria, considerada no magnética (Blanco et al., 2003), como es el caso del volcán en estudio, Auca Mahuida. La interpretación de estos contrastes, junto con el modelado de las fuentes, permite obtener valiosa información sobre características estructurales tales como la geometría de los sistemas de conductos, fallas y zonas de alteración, en el contexto geológico y geofísico existente (Finn et al., 2001; De Ritis et al., 2010).

En regiones volcánicas, los datos aeromagnéticos son útiles para tales propósitos, especialmente cuando otros métodos geofísicos son poco efectivos, como por ejemplo los datos sísmicos, que son difíciles de adquirir sobre topografía muy irregular y con frecuencia tienen baja relación señal-ruido; o en los casos en que los cuerpos fuente son muy pequeños para ser detectados mediante gravimetría, o tienen bajo contraste de densidad con las rocas predominantes.

La profundidad de investigación de un estudio geofísico está limitada por las dimensiones del relevamiento; y en el caso particular de un muestreo magnetométrico, además, por la temperatura del subsuelo, dado que los minerales magnéticos pierden la mayor contribución a la magnetización a una temperatura característica de cada mineral, conocida como temperatura de Curie.

La complejidad geológica- estructural asociada a este volcán y el interés de carácter económico desde el punto de vista petrolero, han dado lugar a numerosos estudios y a la propuesta de diversos modelos que explican la evolución tectónica de la zona. En este contexto, el presente trabajo incorpora el análisis de datos magnéticos y gravimétricos de alta resolución cuya interpretación revela que la configuración de los cuerpos intrusivos del sistema de alimentación del volcán está controlada por el patrón estructural local y regional. 


\subsection{Descripción de la zona de estudio}

El volcán Auca Mahuida (VAM) se ubica en el extremo sur de la provincia volcánica de Payenia en el retroarco (Cuenca Neuquina, Argentina). Dicha provincia está integrada por más de 800 conos basálticos monogénicos y por unos pocos conos poligénicos compuestos por rocas diferenciadas y alimentados desde cámaras magmáticas superficiales como es el caso del VAM. La alineación de conos permite inferir que muchas de las erupciones son de tipo fisural, con un rumbo frecuente ONO oblicuo al eje de la cordillera (Llambías et al., 2010).

La cuenca Neuquina aloja un sistema hidrocarburífero importante, en el cual el magmatismo Plio-Cuaternario ha jugado un papel importante en la formación y acumulación de los hidrocarburos, los cuales tienen un origen principalmente termogénico en el sector del Auca Mahuida (Rossello et al., 2002). Las rocas madre en esta cuenca son sedimentarias, y han estado sometidas a repetidas intrusiones que provocaron aumentos de temperatura y por lo tanto tuvieron un papel central en la generación del petróleo. Por su parte, las rocas reservorio sedimentarias donde se alojó el petróleo poseen dentro de la secuencia sedimentaria cuerpos ígneos fracturados durante su enfriamiento, que pueden ser reservorios.

Los cuerpos ígneos más antiguos, gran parte de ellos intruídos en la Formación Quintuco - Vaca Muerta, tienen entre 24 y 12 M.a. (Mioceno). Por otra parte, las rocas ígneas intruídas en los niveles superiores, más cercanos a la superficie, son más jóvenes, entre 10 y 2.5 M.a. (Mioceno- Plioceno), mientras que el vulcanismo fisural extrusivo ha sido datado entre 2.4 y 0.8 M.a. (Plioceno-Pleistoceno).

\subsection{Objetivos}

El objetivo del trabajo de tesis propuesto, consiste en obtener un mayor conocimiento de las estructuras internas del complejo volcánico fisural Auca Mahuida y su relación con el marco geodinámico regional. Esto se efectuó mediante el análisis y modelado de los datos magnéticos y gravimétricos integrados con información de otras disciplinas involucradas en la caracterización geológica del área. La información disponible de perfiles y muestras de roca proveniente de perforaciones (coronas y cutting), posibilitó la obtención de mediciones de densidad, velocidades dentro de una formación, dataciones radimétricas, petrología, así como valores de temperatura a distintas profundidades, los que fueron utilizados a lo largo de esta tesis. Los datos provenientes de relevamientos sísmicos 2D y 3D, han sido utilizados fuera de la zona del cráter central para visualizar las estructuras ígneas intruidas como filones capa en la secuencia sedimentaria.

La determinación de las profundidades de las fuentes es de vital importancia para poder afirmar que el campo de anomalías magnéticas observado no se origine principalmente en las lavas superficiales. 
Como parte de la investigación realizada se llevó a cabo una campaña en el plateau volcánico del Auca Mahuida, donde se midió la susceptibilidad magnética in situ y se recolectaron muestras. A posteriori se determinaron las propiedades magnéticas de las principales unidades volcánicas en el laboratorio del Istituto Nazionale di Geofisica e Vulcanologia di Roma (INGV).

Debido a los grandes espesores de roca ígnea presentes en superficie se hizo un cuidadoso análisis para diferenciar las señales provenientes de las estructuras profundas de las someras, basado en las siguientes técnicas:

i. análisis espectral para identificar las principales longitudes de onda de la región (Naidu \& Mathew, 1998);

ii. estudio del efecto topográfico en el edificio del Auca Mahuida;

iii. técnicas de interpretación analítica y numérica 2D, $2.75 \mathrm{D}$ y 3D para determinar las geometrías de las fuentes y su relación con el marco tectónico.

Por último, para completar una interpretación confiable de los campos de anomalías del VAM, se trabajó con métodos de inversión, obteniendo un valor de magnetización representativo. Este resultado proveyó restricciones adicionales a los modelos estructurales directos 2.75D, en los cuales se usaron además valores de magnetización y remanencia medidos en campo y laboratorio.

\subsection{Estructura de la tesis}

Esta tesis se ha estructurado en siete capítulos como se detalla a continuación. El Capítulo I es la presente introducción. El Capítulo II es una revisión de los antecedentes del área y una descripción del marco geológico del volcán de estudio. Se incluye una reseña de los principales reservorios de hidrocarburos del área. En el Capítulo III se describen los datos utilizados, haciendo hincapié en los datos magnéticos. Además, se detalla la secuencia de tratamiento de los datos magnéticos y gravimétricos, desde su adquisición en el avión hasta la obtención del mapa de anomalías magnéticas y gravimétricas respectivamente. En el Capítulo IV se desarrollan los aspectos teóricos de la magnetización de las rocas que da origen a las anomalías magnéticas. Por otro lado se hace referencia a las mediciones paleomagnéticas de las muestras obtenidas en el plateau volcánico, cuyos resultados dieron lugar a parámetros de entrada en varias técnicas de análisis. En el Capítulo V, se analizan las anomalías magnéticas del área de estudio y se desarrollan algunas de las técnicas más utilizadas en los campos potenciales que resultan más apropiadas para las características del campo de anomalías que nos ocupa (fuerte remanencia de polaridad reversa con respecto al campo ambiente). Luego se exponen los resultados obtenidos de la aplicación de estos métodos a los datos del volcán en estudio. En el Capítulo VI se trabaja e integran los datos de gravedad, sísmica y de perforaciones, los cuales contribuyen a una interpretación más realista de las estructuras del edificio volcánico. En el Capítulo VII se construyen modelos 
sobre perfiles estratégicamente elegidos sobre los mapas de anomalías magnéticas y gravimétricas. Se realiza además, un modelo de inversión 3D de las anomalías magnéticas para calcular el vector de magnetización. Finalmente, en el Capítulo VIII se hace una discusión general de los resultados presentados a lo largo de la tesis y de las ventajas y limitaciones encontradas en la implementación de las técnicas de interpretación utilizadas. Por último se exponen las conclusiones generales. 


\section{CAPÍTULO II}

\section{MARCO GEOLÓGICO DEL VOLCÁN AUCA MAHUIDA}

El conocimiento de la geología de una determinada zona de estudio es el punto de partida para realizar estudios geofísicos. Las campañas de adquisición de datos geofísicos y la selección de métodos para su análisis se pueden llevar a cabo en forma óptima si se tiene una adecuada comprensión previa del marco geológico. En el presente caso de estudio, numerosos trabajos describen el contexto geodinámico del sistema volcánico Auca Mahuida, un área de gran complejidad, en la cual la existencia de reservorios de hidrocarburos, ha incrementado el interés debido a diversos aspectos que resultan de su caracterización.

\subsection{Antecedentes de estudios de volcanes con métodos potenciales}

En esta sección se hace una recopilación de algunas publicaciones en las cuales se utilizaron datos magnetométricos y gravimétricos para caracterizar estructuras volcánicas. En especial, los datos aeromagnéticos son usados para evaluar y analizar las propiedades magnéticas y estructuras geológicas asociadas a volcanes por los altos contrastes de susceptibilidad magnética y magnetización presentes en rocas ígneas.

En la tabla 2.1 se hace un resumen de los resultados obtenidos de la aplicación del uso de datos gravimétricos y magnéticos en los siguientes volcanes:

- Isla volcánica Salina (De Ritis et al., 2007),

- Isla volcánica El Hierro (Blanco et al., 2008),

- Complejo volcánico Colima (López Loera et al., 2011) y

- Volcán Galeras (Ortega Estupiñán, 2014). 
Tabla 2.1. Estructuras asociadas con el origen de anomalías magnéticas y gravimétricas en algunos volcanes previamente estudiados.

\begin{tabular}{|c|c|c|}
\hline Estudio & Tipo de Datos & Características y resultados \\
\hline $\begin{array}{l}\text { Isla volcánica } \\
\text { Salina } \\
\text { De Ritis et al., } 2007\end{array}$ & $\begin{array}{l}\text { Relevamiento aeromagnético } \\
\text { barométrico de baja altitud } \\
(500 \mathrm{~m}) \text { en la isla Salina (Italia). }\end{array}$ & $\begin{array}{l}\text { Se asociaron las anomalías } \\
\text { positivas de longitud de onda corta } \\
\text { con conductos volcánicos, diques y } \\
\text { fallas. Anomalías negativas } \\
\text { regionales han servido para definir } \\
\text { el colapso del sector y rodean a las } \\
\text { anomalías positivas. }\end{array}$ \\
\hline $\begin{array}{l}\text { Isla volcánica, El } \\
\text { Hierro } \\
\text { Blanco et al., } 2008\end{array}$ & $\begin{array}{l}\text { Relevamiento aeromágnético } \\
\text { registrado en } 7 \text { bloques a } \\
\text { distintas altitudes con } \\
\text { resolución espacial variable } \\
\text { sobre la isla El Hierro } \\
\text { (Canarias) y áreas circundantes. }\end{array}$ & $\begin{array}{l}\text { Anomalía dipolar normal intensa. } \\
\text { Se interpretaron estructuras } \\
\text { verticales magnetizadas como } \\
\text { complejos intrusivos en una } \\
\text { estructura compleja de rift. Zonas } \\
\text { de baja magnetización fueron } \\
\text { interpretadas como grandes } \\
\text { deslizamientos de terreno. }\end{array}$ \\
\hline $\begin{array}{l}\text { Complejo volcánico } \\
\text { Colima } \\
\text { López Loera et al., } \\
2011\end{array}$ & $\begin{array}{l}\text { Integración de tres } \\
\text { relevamientos aeromagnéticos } \\
\text { llevados a cabo con distinto } \\
\text { instrumental. Altura de vuelo } \\
\text { promedio para las tres } \\
\text { campañas es de } 300 \mathrm{~m} \text { sobre } \\
\text { nivel del terreno. }\end{array}$ & $\begin{array}{l}\text { Anomalías magnéticas de distintas } \\
\text { longitudes de onda. Se dividió en } \\
\text { dominios magnéticos. Los } \\
\text { principales correspondientes a los } \\
\text { volcanes Colima y Nevado están } \\
\text { asociados con anomalías } \\
\text { magnéticas dipolares normales de } \\
\text { gran amplitud. Se identificaron } \\
\text { zonas correlacionadas con rocas } \\
\text { ígneas intrusivas, extrusivas, } \\
\text { volcano-sedimentarias y calcáreas. } \\
\text { Los lineamientos magnéticos } \\
\text { permitieron inferir algunos } \\
\text { aspectos de la estructura del } \\
\text { subsuelo del complejo volcánico de } \\
\text { Colima. }\end{array}$ \\
\hline $\begin{array}{l}\text { Volcán Galeras } \\
\text { Ortega Estupiñán, } \\
2014\end{array}$ & $\begin{array}{l}\text { Medición de puntos } \\
\text { gravimétricos y } \\
\text { magnetométricos, } 286 \\
\text { estaciones. }\end{array}$ & $\begin{array}{l}\text { Bajos gravimétricos, uno en la zona } \\
\text { del cráter, representados por } \\
\text { bloques de baja densidad en los } \\
\text { modelos } 2 \mathrm{D} \text {. Altos gravimétricos, se } \\
\text { destaca un intrusivo asociado con } \\
\text { zona de alta velocidad de ondas P. } \\
\text { Conducto volcánico o zona de falla. }\end{array}$ \\
\hline
\end{tabular}


Entre los trabajos consultados podemos citar además, las tres calderas volcánicas, Decepción, Furnas y Las Cañadas del Teide, estudiadas por Blanco (1997) en su tesis doctoral; las calderas Fossa y Piano de la isla Vulcano (Blanco et al., 2006) y la caldera de Pacana estudiada por Delgado de la Puente (2010) en su tesis de licenciatura mediante datos gravimétricos terrestres.

Blanco (1997), menciona en su tesis doctoral que la respuesta gravimétrica en calderas volcánicas puede presentarse como un máximo o un mínimo en las anomalías de Bouguer dependiendo de su relleno. Así, la existencia de un mínimo gravimétrico se vincula con calderas rellenadas por grandes cantidades de material fragmentario, asociadas a magmas ácidos, mientras que valores positivos en la anomalía de Bouguer están relacionados con calderas asociadas a la erupción de magmas básicos. Maturyono et al., 2001, estudiaron el complejo caldera Toba, cuya respuesta gravimétrica son valores bajos en la anomalía de Bouguer, alcanzando los -75 mGal. Ellos explican ese mínimo como resultado de tres factores posibles: 1- compensación isostática de la elevación topográfica del área, 2- estrato de roca tobácea cuya densidad es más baja que la de las rocas aledañas, y 3- dismución de la densidad de las rocas por expansión térmica.

\subsection{Marco geológico del Volcán Auca Mahuida}

El escudo volcánico Auca Mahuida (VAM) se ubica en el sector noreste de la Cuenca Neuquina, Patagonia norte. Está formado por una meseta de lava elongada en sentido E-O (Ventura et al., 2012) con un cono central poligénico ( $37^{\circ} 44^{\prime} \mathrm{S}, 68^{\circ} 55^{\prime}$ 0) que alcanza una altitud de 2258 m sobre el nivel del mar (Holmberg, 1964; Rossello et al., 2002), y conos monogénicos que se concentran en la zona de la cima y en los flancos oriental y occidental, alineados con fisuras de rumbo E-O. En esta zona la densidad de conos monogénicos es mayor a cinco conos por cada $4 \mathrm{~km}^{2}$. El plateau cubre una superficie de alrededor de $75 \times 62$ $\mathrm{km}^{2}$. Los espesores de lava alcanzan los $400 \mathrm{~m}$ debajo del cráter central y disminuyen prácticamente a cero en la periferia de la meseta de lava según indican los datos de perforaciones (Rossello et al., 2002; Longo et al., 2008). La topografía y el análisis de las pendientes presentado por Ventura et al. (2012) muestran un cono central de unos $15 \mathrm{~km} \mathrm{x}$ $11 \mathrm{~km}$ elongado en dirección E-O con pendientes de 8 a $10^{\circ}$ en promedio y valores máximos de $30^{\circ}$. El complejo volcánico está caracterizado por laderas suaves, generalmente de menos de $5^{\circ}$. La parte central de este cono es un cráter sub-circular de $1.8 \mathrm{~km}$ de diámetro. Estructuras lobulares correspondientes a los frentes y lados de los flujos de lava caracterizan las laderas del cono así como también el plateau del Auca Mahuida en su conjunto. Las laderas más empinadas del cono, en el sector central del complejo, son flujos de lava traquíticos, comprobado por el relevamiento de campo realizado en este trabajo de tesis.

El volcán Auca Mahuida es el edificio más austral de la provincia volcánica de 
retroarco de Payenia, la cual se extiende en el antepaís andino entre los $35^{\circ}$ y $38^{\circ}$ de latitud sur (Ramos \& Kay, 2006; figura 2.1), y está ubicado alrededor de $400 \mathrm{~km}$ al este de la cordillera de los Andes. La actividad volcánica con expresión superficial, se desarrolló entre los 2.4 y 0.8 M.a. (Plioceno Superior - Pleistoceno) y la composición de las rocas varía entre basaltos alcalinos de intra-placa y traquitas (Kay et al., 2006; Ramos \& Folguera, 2010). Dataciones radimétricas de los intrusivos (Bermúdez \& Delpino, 1998) indican que el área de estudio experimentó tres ciclos magmáticos principales: 25-20 M.a. (Oligoceno - Mioceno inferior; parte del sustrato del volcán pertenece a esta fase), 15-10 M.a. (Mioceno medio) y 5 M.a. (Mioceno superior - Pleistoceno inferior). Trabajos recientes (Pallares, et al., 2016) proponen a partir del estudio de 23 muestras extraídas en la porción central y sur del casquete volcánico del Auca Mahuida que hubo cinco fases de actividad volcánica principales entre $1.78 \pm 0.10$ a $0.99 \pm 0.04 \mathrm{Ma}$. Excepto para la fase más vieja, de la cual podría no haber lavas basales en estas muestras, cada una de ellas se inicia por la emisión de traquibasaltos de alto contenido de $\mathrm{MgO}$, los cuales evolucionaron a productos más diferenciados tales como traquitas o traquiandesitas.

Los principales eventos ígneos ocurrieron bajo regímenes extensionales que generaron fracturas profundas, las cuales son necesarias para el ascenso de los fluidos desde grandes profundidades. Esta fase extensional se alternó con eventos compresionales dando origen a la construcción de la faja plegada. El vulcanismo de Payenia está ausente al sur del volcán Auca Mahuida, donde el lineamiento de Cortaderas de rumbo $100^{\circ} \mathrm{E}$, marca el límite austral de una subducción somera del Mioceno (Kay et al., 2006). Este límite se traduce en la superficie como fallas de rumbo $\mathrm{N} 100{ }^{\circ}$ a E-O afectando la depresión de Añelo (figura 2.1).

El campo volcánico del Auca Mahuida se desarrolla sobre un anticlinal de rumbo NNO-SSE a NO-SE involucrando la secuencia sedimentaria completa de la cuenca Neuquina (Rossello et al., 2002; Mosquera \& Ramos, 2006). Esta secuencia está afectada por fallas normales de rumbo NNO-SSE a NO-SE del hemi-graben de Entre Lomas (Cristallini et al. 2006). La actividad extensional de estas fallas se desarrolló principalmente en los períodos Jurásico Tardío a Cretácico Superior, con menores niveles de actividad en tiempos más recientes.

La columna estratigráfica del área está formada por una serie de depósitos marinocontinentales con edades comprendidas entre el Triásico Superior y el Terciario Inferior. Las principales formaciones sedimentarias se resumen en la figura 2.2. El basamento cristalino subyace a una profundidad de unos $5 \mathrm{~km}$ (Zapata \& Folguera, 2005) el cual está afectado por fallas de rumbo N-S a NO-SE.

Desde el punto de vista magnético, la estratigrafía local se puede subdividir en cuatro unidades principales: un basamento magnético (rocas volcánicas, plutónicas y metamórficas del Paleozoico inferior a Triásico Superior); la secuencia sedimentaria no magnética (rocas carbonáticas, evaporíticas y clásticas del Triásico a Terciario inferior); rocas magnéticas intrusivas y del plateau basáltico superficial, del Plioceno-Pleistoceno. Estas intrusiones son principalmente filones capa concordantes o no con la estratigrafía. 


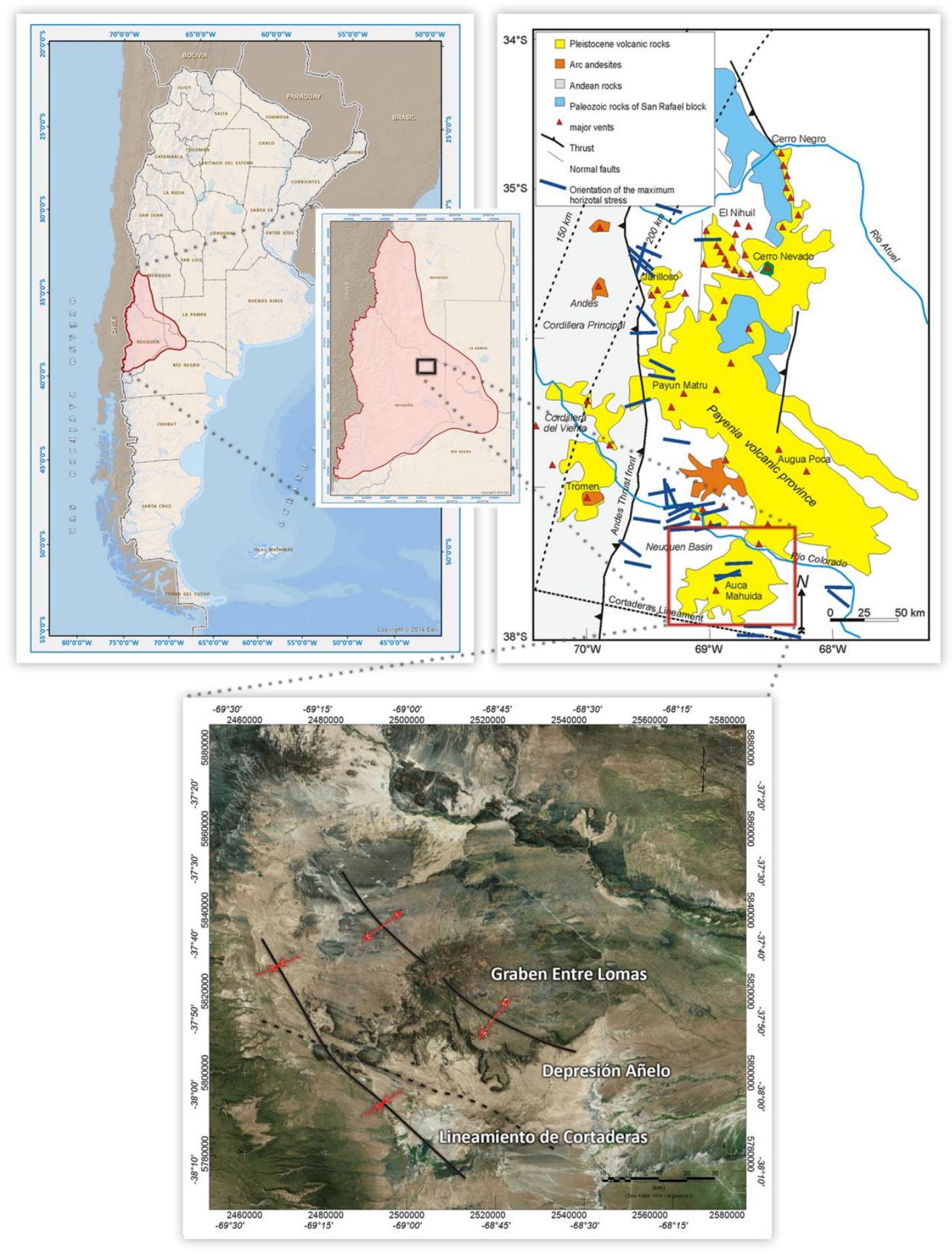

Figura 2.1. Arriba izquierda: ubicación de la Cuenca Neuquina (contorno rojo sobre el mapa de Argentina) junto con el plateau Auca Mahuida dentro de la cuenca. Arriba derecha: mapa esquemático de la provincia volcánica de Payenia. Abajo: imagen satelital del complejo volcánico con indicación de los principales rasgos estructurales. 


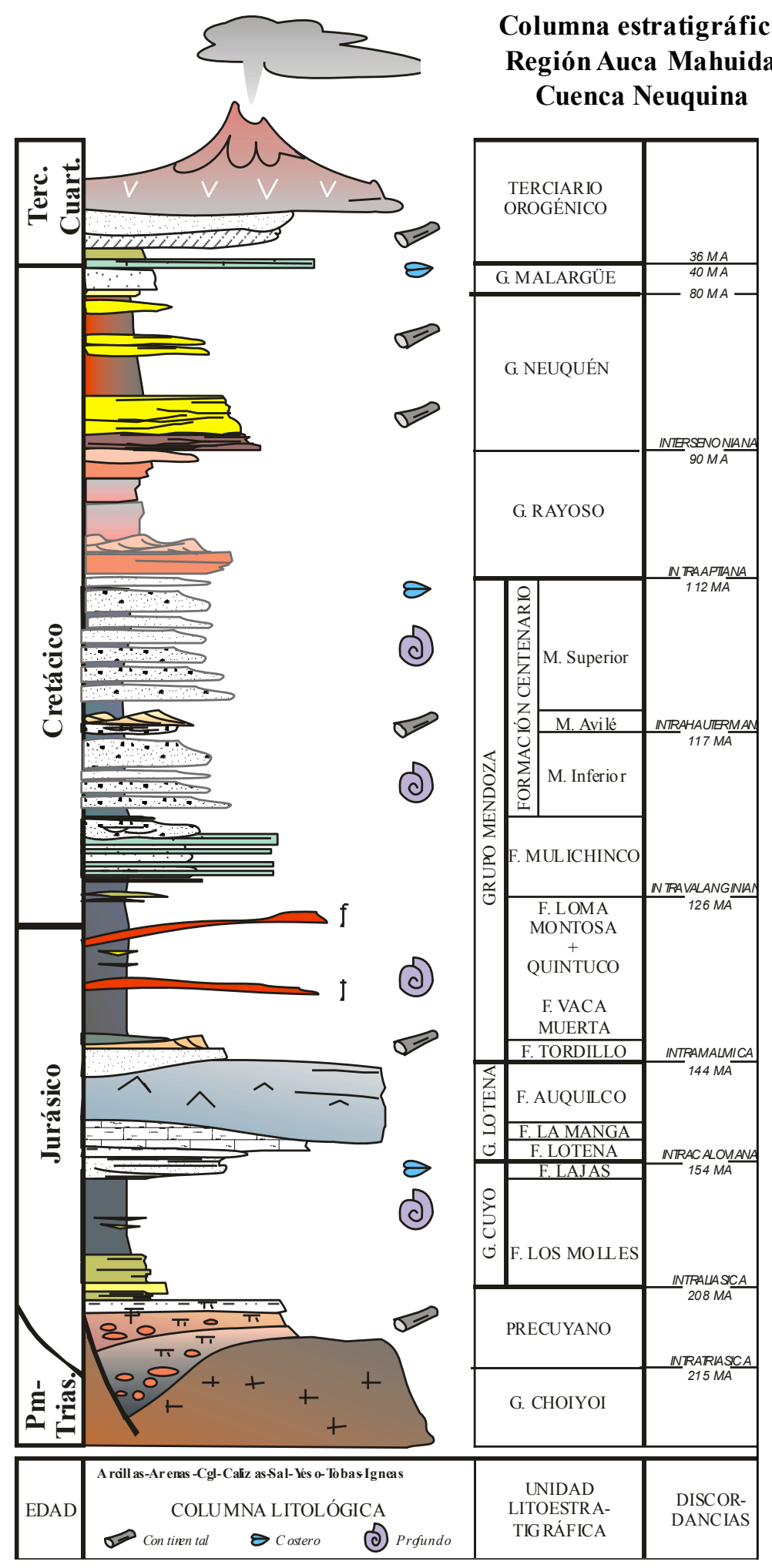

Figura 2.2. Columna estratigráfica correspondiente al área del volcán Auca Mahuida (Brisson \& Veiga, 1999). 


\subsection{Tipos de rocas y caracterización geoquímica}

Los análisis petrológicos realizados en rocas ígneas, proveen una herramienta esencial para el conocimiento de la evolución tectónica de una zona. La composición geoquímica de las rocas del área de estudio ha permitido hacer hipótesis sobre el origen y emplazamiento del volcán Auca Mahuida.

Las rocas plutónicas analizadas, de acuerdo a su contenido de sílice son básicas a intermedias. Las primeras comprenden a dos tipos litológicos: gabros y sieno-dioritas; las intermedias incluyen un solo tipo litológico: sienitas. Las rocas volcánicas y subvolcánicas (cuerpos intrusivos) relacionadas con el VAM y el campo volcánico adyacente, son en cuanto a su contenido de sílice, básicas e intermedias. Las rocas básicas comprenden los tipos litológicos de basaltos y traquibasaltos, y las intermedias las traquiandesitas basálticas, traquiandesitas y traquitas. Se ha observado que la mayor cantidad de cuerpos intrusivos se alojan en la sección inferior de las Formaciones Quintuco y Vaca Muerta, (posiblemente por ser niveles compuestos por pelitas que facilitan la intrusión desde el punto de vista mecánico) y en menor medida en las Formaciones Mulichinco, Centenario, Rayoso y el Grupo Neuquén (Bermúdez \& Delpino, 1998, 2002).

De acuerdo a la composición geoquímica de las rocas estudiadas, Bermúdez y Del Pino, (1998) y Bermúdez et al., (2000) pudieron establecer que el volcán se habría originado a partir de fundidos litosféricos resultantes de la fusión parcial de la placa oceánica subductada en contacto con la astenósfera. Estos eventos de fusión pueden estar asociados al aumento de la pendiente de la losa subducida previamente horizontal, la cual habría producido un ascenso astenosférico (Kay et al., 2004, Pallares et al., 2016, figura 2.3). Estos fundidos ascendieron hasta la base de la corteza continental y evolucionaron en una o varias cámaras magmáticas. Luego el magma se elevó a profundidades más someras (6-10 km) debajo del área del VAM, donde los fundidos se homogeneizaron sufriendo procesos de diversificación magmática dando lugar a las rocas más ácidas de la serie. Las que alcanzaron la superficie son las traquitas que forman parte del VAM, mientras que las rocas intrusivas equivalentes corresponden a filones capa de composición traquítica y sienítica. Las rocas extrusivas basálticas y traquibasálticas relacionadas con los conos monogénicos del campo volcánico adyacente al volcán y los intrusivos que forman filones capa de composición gábrica, ascendieron directamente desde las cámaras relativamente más grandes situadas en la base de la corteza y desde las más pequeñas en conexión directa con la astenósfera. Los principales eventos ígneos se produjeron bajo regímenes extensionales que generaron fracturas profundas, las cuales se requieren para el ascenso de los fundidos desde grandes profundidades. Los mecanismos descriptos implican que las rocas han tenido una fuente de origen común y los distintos tipos litológicos se han originado a partir de procesos de diversificación magmática; en este caso particular el proceso más probable es el de cristalización fraccionada, desarrollado dentro de una o varias cámaras magmáticas pero que han evolucionado bajo similares condiciones físicas y químicas. 


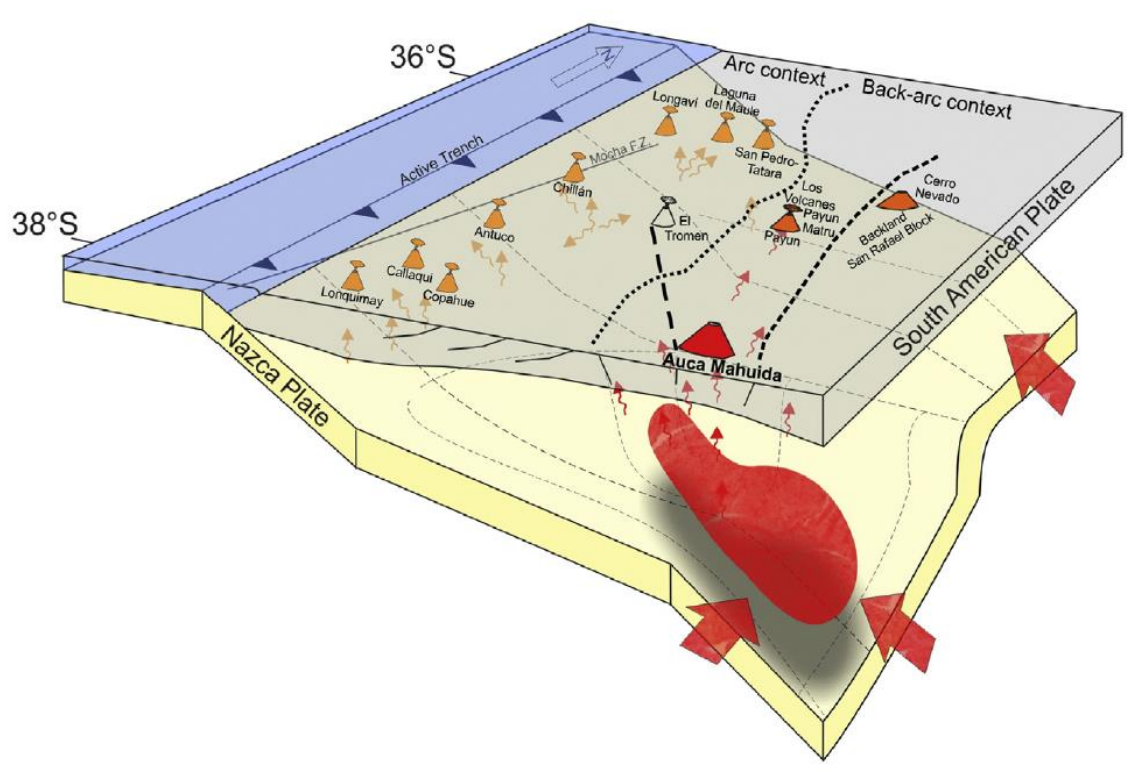

Figura 2.3. Modelo esquemático propuesto para el origen de las lavas de retroarco dentro de la provincia volcánica de Payenia. Se muestra la placa oceánica deformada y la consecuente surgencia de material astenosférico. Figura tomada de Pallares et al. (2016).

\subsection{Reservorios de hidrocarburos}

La exploración hidrocarburífera en el área comenzó en 1985 con la perforación del pozo VAM.x-1 y continuó en la década siguiente con el VAM.x-2. A partir de la información obtenida de estos dos pozos, se perforó el VAM.x-3 el cual resultó descubridor de hidrocarburo en un nivel arenoso a una profundidad de $2470 \mathrm{mbbp}$ (metros bajo boca de pozo). De este modo, se desarrolla el yacimiento del VAM en el noreste del escudo volcánico (figura 2.4). La estructura que conforma el yacimiento ha sido generada por la intrusión de filones capa principalmente en las Formaciones Quintuco y Vaca Muerta, como así también en niveles más profundos del Grupo Cuyo. Algunos de estos emplazamientos ígneos son concordantes a varios niveles y otros lo son discordantes a la estratificación. Localmente, aparecen filones que atraviesan la Formación Mulichinco formando altos puntuales al desplazar la columna verticalmente. Estos filones están vinculados a fallas subverticales, que son o bien producto de la estructuración asociada a la intrusión de éstos cuerpos ígneos, o anteriores a la misma, y es posible que en varios casos constituyan el canal alimentador de los intrusivos alojados en la columna.

El conocimiento de la geología, el control estructural de las erupciones, la obtención de las edades absolutas y la caracterización geoquímica de las rocas, permiten aproximar algunas posibles relaciones entre los procesos que controlaron la actividad volcánica y la generación y migración de hidrocarburos.

La roca madre del petróleo alojado en el yacimiento VAM pertenece a la Formación 
Vaca Muerta (Pángaro et al., 2004). Esta unidad, junto a la Formación Quintuco conforman un arreglo progradante que abarca desde términos de off-shore (margas y arcilitas) hasta calizas de plataforma interna. El límite entre ambas unidades es de carácter lito-estratigráfico.

El reservorio del yacimiento está constituido por las areniscas marinas litorales a fluviales distales de la Formación Mulichinco (Vottero, 2002). Su porosidad promedio es del $18 \%$ superando, en algunos caos, el $22 \%$. Estas arenas se caracterizan por una gran continuidad areal y por presentarse como cuerpos con una disposición aproximadamente tabular, separados por niveles impermeables que representan, en general, eventos de exposición subaérea. Inmediatamente sobre la Formación Mulichinco se ubica un paquete arcilloso de unos 25 m de espesor, asociado a la transgresión marina basal de la Formación Agrio; éste constituye un sello de alcance regional mientras que los niveles arcillosos de la Formación Mulichinco constituyen sellos locales.

La trampa es netamente estructural y el yacimiento se encuentra restringido, por el momento, a su flanco NE.

Una de las hipótesis para explicar la presencia de hidrocarburos en el yacimiento, es que el evento volcánico y el emplazamiento de filones, además de originar la estructura, tuvo asociado un régimen de alto flujo calórico que afectó a la Formación Vaca Muerta causando una generación extra de petróleo. Éste migró a través de fallas verticales hasta los reservorios de la Formación Mulichinco.

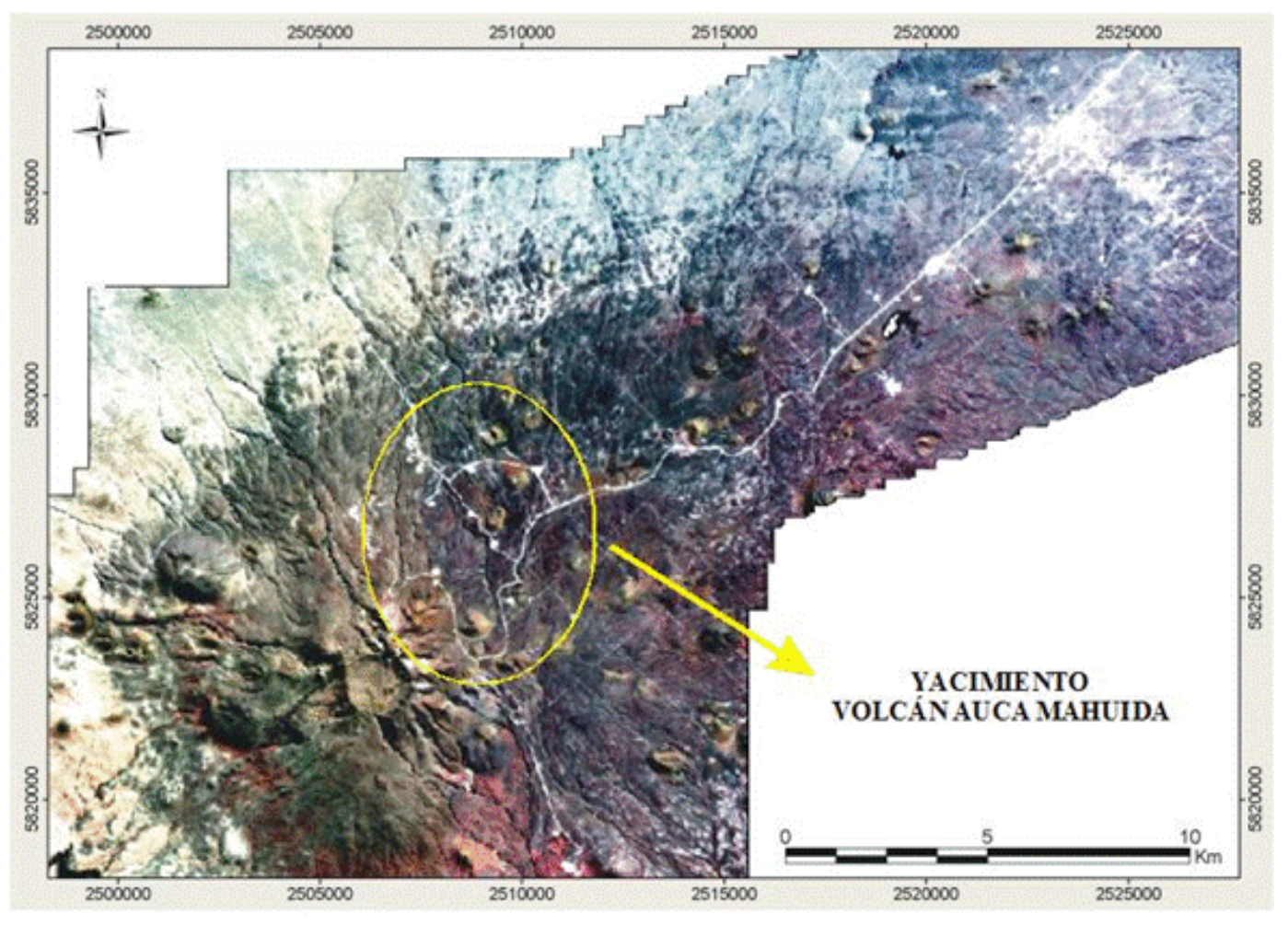

Figura 2.4. Imagen Satelital IKONOS, del año 2002, resolución $1 \mathrm{~m}$. En el óvalo amarillo se muestra la localización del yacimiento Volcán Auca Mahuida. 


\section{CAPÍTULO III}

\section{DESCRIPCIÓN Y TRATAMIENTO DE LOS DATOS}

En este capítulo se hace una revisión de los datos con los que se trabajó en esta tesis. Se incluyen datos geofísicos -sísmicos, magnéticos, gravimétricos-, mediciones paleomagnéticas, datos de pozo -litología, estratigrafía, perfiles (especialmente sónico y densidad), temperatura de fondo y pases formacionales. Se analizó la calidad de los mismos y las limitaciones que presentan algunos de ellos debido a los espesores de rocas volcánicas existentes en superficie en la mayor parte del área de estudio. La influencia de estos factores en la relación señal-ruido es de particular importancia en el análisis e interpretación de los datos. Los datos aeromagnéticos, datos de topografía, modelo digital de elevaciones (DEM por sus siglas en inglés), imágenes Landsat e Ikonos, así como también toda la información referente a datos de pozos fueron provistos por YPF S. A. Las mediciones paleomagnéticas de las muestras tomadas en el campo fueron realizadas en el laboratorio del Istituto Nazionale di Geofisica e Vulcanologia (INGV) y las muestras de pozo en el Laboratorio de Paleomagnetismo Daniel Valencio de la Universidad de Buenos Aires.

\subsection{Base de datos}

Se compiló una base de datos geográfica única en el software ArcGIS (Geographic Information System), con información de las anomalías magnéticas, litología, imágenes satelitales, DEM (figura 3.1), datos de pozo, para realizar una correlación espacial y confección de mapas. Además, gran parte de estos datos se volcó en la aplicación Oasis Montaj del software Geofsoft ${ }^{\mathrm{TM}}$. La disponibilidad de todos los datos reunidos en la base GIS como en Oasis Montaj, permitió hacer un análisis comparativo y correlaciones entre rasgos geológicos y geofísicos. El sistema de referencia usado a lo largo de este trabajo de tesis es Chos Malal, y la proyección Gauss Kruger faja 2. Todos aquellos datos cuyo datum difiere del elegido, fueron transformados antes de ser utilizados.

Por otro lado, se confeccionaron tablas con información brindada por los datos de pozos disponibles en el área de trabajo, como ser, estratigrafía, temperatura de fondo y a otras profundidades, espesores de basaltos, topes de las formaciones que componen la 
columna sedimentaria. Toda esta información ordenada permitió construir los pilares de este trabajo de tesis.

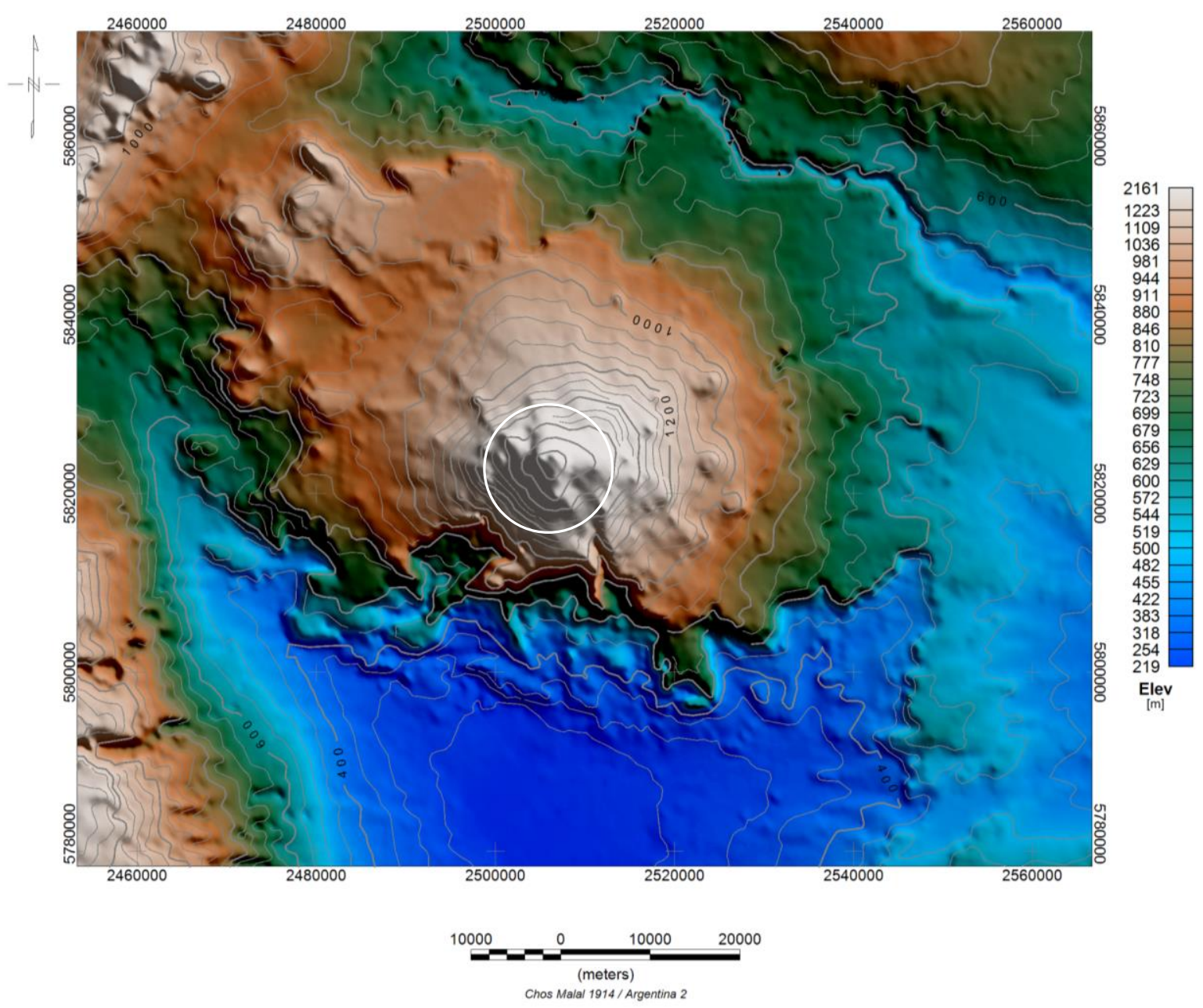

Figura 3.1. Mapa del modelo de elevación digital del terreno del Volcán Auca Mahuida provisto por YPF S A. Las unidades están dadas en m sobre nivel del mar. Resolución: 90 m x 90 m. En círculo blanco se indica el cráter central.

\subsection{Adquisición y reducción de los datos magnéticos y gravimétricos en el volcán Auca Mahuida}

Con el objetivo de obtener mayor información del subsuelo en esta zona, se llevó a cabo un relevamiento magnético y gravimétrico aéreo de alta resolución en el año 2001 por la empresa Carson Aerogravity para YPF S. A. Se adquirieron 3436 y 2889 kilómetros de datos magnéticos y gravimétricos respectivamente, en los bloques “Volcán Auca Mahuida” y "Señal Cerro Bayo", comprendidos entre $37^{\circ} 33^{\prime}$ y $37^{\circ} 51^{\prime}$ de latitud sur, y $68^{\circ} 37^{\prime}$ y $69^{\circ} 06^{\prime}$ de longitud oeste (figura 3.2). El relevamiento consta de 113 líneas, de las cuales 53 fueron 
recorridas con acimut de 90 o 270 grados medidos desde el norte; y 60 con acimut de 0 o 180 grados desde el norte. Estos datos fueron medidos desde un helicóptero Sikorsky Twin Engine S-61 volando a una velocidad de 50 nudos ( $92.6 \mathrm{~km} / \mathrm{h}$ aproximadamente) a una altura promedio de vuelo de $2830 \mathrm{~m}$ sobre nivel del mar. Las mediciones fueron realizadas a intervalos de 1 segundo, con lo cual los puntos de medición están separados una distancia de $25 \mathrm{~m}$. El área se recorrió usando una grilla de vuelo con espaciamientos de $2 \mathrm{~km} \mathrm{x} 2 \mathrm{~km}$, excepto en la parte central del bloque del relevamiento donde para lograr mayor detalle se usó una grilla de $0.5 \mathrm{~km}$ x $0.5 \mathrm{~km}$. Se realizaron 52 vuelos entre marzo y junio de dicho año, siendo los primeros para pruebas, calibraciones de los equipos de medición, etc. Hubo vuelos suspendidos por causas tales como excesiva turbulencia o malas condiciones de vuelo.

La navegación del helicóptero se realizó con un GPS NAVSTAR y con un sistema de posicionamiento de tiempo real. La altimetría se midió con un transductor de presión Rosemount modelo $1201 \mathrm{~F}$ el cual posee un sistema de compensación de temperatura para reducir los efectos de las variaciones de temperatura ambiente. Por otra parte, para medir la altitud respecto al terreno, el helicóptero usó un altímetro de radar pulsado que mide el objeto más cercano a la nave.

Para medir la intensidad del campo magnético total se usó un magnetómetro "Geometrics High Sensitivity Cesium Vapor", cuya precisión es del orden de $\pm 0.15 \mathrm{nT}$. El sensor del magnetómetro se colgó del helicóptero por medio de un cable a una distancia tal que el detector se mantuviera libre de la influencia magnética de la aeronave.

Las mediciones gravimétricas fueron hechas con un gravímetro "La Coste \& Romberg Air/Sea Gravity Meter (Model S)", del tipo de resorte montado sobre plataforma estabilizada con giróscopos, cuya precisión estática es de $0.01 \mathrm{mGal}$.

El procesamiento de los datos magnéticos y gravimétricos realizado por la empresa Carson Aerogravity, fue llevado a cabo para obtener el campo de anomalías de intensidad magnética total y el campo de anomalías gravimétricas, respectivamente. 


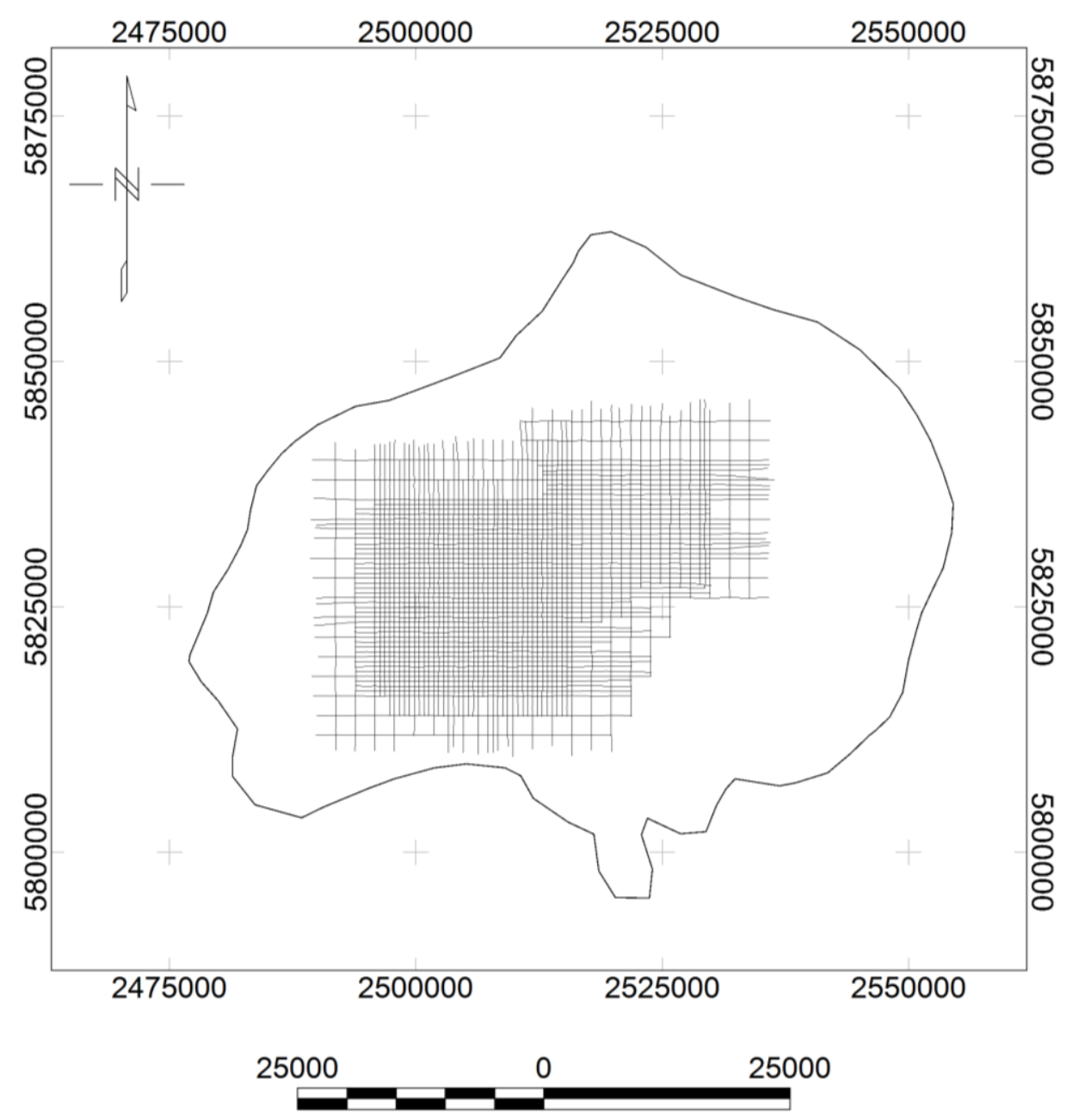

$(\mathrm{m})$

Chos Malal 1914 / Argentina 2

Figura 3.2. Patrón de líneas de vuelo del relevamiento aeromagnético-gravimétrico 2001 en color negro. Contorno: plateau volcánico. Se puede apreciar que el relevamiento aeromagnético no cubre todo el plateau.

\subsubsection{Generalidades sobre Campo geomagnético}

El campo magnético terrestre es la resultante de varias componentes magnéticas, originadas dentro y fuera de la Tierra, las cuales varían espacial y temporalmente sobre el planeta. El campo principal generado por corrientes electromagnéticas en el núcleo exterior constituye alrededor del $98 \%$ del campo geomagnético. Los campos de origen externo contribuyen a la mayor parte del campo restante. Este campo externo es debido principalmente a la actividad del Sol sobre la ionósfera y la magnetósfera, y presenta variaciones periódicas siendo la más importante la variación diaria con período de 24 horas. También son periódicas la variación lunar, la variación anual y la variación undecenal. Otras 
variaciones rápidas de origen externo son las pulsaciones magnéticas, las tormentas magnéticas, las bahías, los efectos cromosféricos, etc. El menor aporte, lo constituyen los efectos estáticos debidos a la magnetización de las fuentes en el subsuelo. A esta última componente del campo, se la conoce como anomalías magnéticas corticales, las cuales se asocian al basamento formado por unidades ígneo-metamórficas y a cuerpos de roca ígnea intruidos en el subsuelo. Las anomalías magnéticas corticales constituyen el principal objeto de estudio de esta tesis.

El campo de inducción magnética se puede expresar como el gradiente de un potencial escalar, esto es, $\boldsymbol{B}=-\nabla V$. Teniendo en cuenta que $V$ es armónico en la región libre de fuentes, es decir, satisface la ecuación de Laplace, $\nabla^{2} V=0, V$ se puede representar por una expansión en armónicos esféricos como:

$$
\begin{aligned}
V & =a \sum_{n=1}^{\infty}\left[\left(\frac{a}{r}\right)^{n+1} T_{n}^{i}+\left(\frac{r}{a}\right)^{n} T_{n}^{e}\right] \\
T_{n}^{i} & =\sum_{m=0}^{n}\left(g_{n}^{m i} \cos m \emptyset+h_{n}^{m i} \operatorname{sen} m \emptyset\right) P_{n}^{m}(\theta) \\
T_{n}^{e} & =\sum_{m=0}^{n}\left(g_{n}^{m e} \cos m \emptyset+h_{n}^{m e} \operatorname{sen} m \emptyset\right) P_{n}^{m}(\theta)
\end{aligned}
$$

donde $a$ es el radio de la Tierra, $r$ la distancia al origen, $\theta$ la colatitud, $\varnothing$ la longitud y $P_{n}^{m}(\theta)$ son los polinomios de Legendre de grado $n$ y orden $m$ normalizados de acuerdo a la convención de Schmidt. $g_{n}^{m i}, g_{n}^{m e}, h_{n}^{m i}, \mathrm{y} h_{n}^{m e}$ son los coeficientes de Gauss que tienen dimensiones de inducción magnética, expresados en general en unidades de nT y pueden ser obtenidos de un análisis del desarrollo de armónicos esféricos (Lowrie, 2007; Blakely, 1995). El término $n=0$ correspondiente al coeficiente $g_{0}^{0}$ no fue tenido en cuenta porque corresponde al potencial de un monopolo.

El primer término de la ecuación (3.1), corresponde a la contribución de origen interno, y el segundo a la externa.

Los coeficientes de la ecuación (3.1) pueden determinarse mediante la relación $\boldsymbol{B}=$ $\nabla V$. Si bien el potencial magnético $V$ no se mide directamente en los estudios geofísicos, los magnetómetros pueden medir el módulo y las componentes ortogonales del campo de inducción.

El vector de campo magnético puede expresarse como componentes en coordenadas esféricas $(B r, B \theta, B \emptyset)$ y también cartesianas $\left(B_{x}, B_{y}, B_{z}\right)$ paralelas a tres ejes ortogonales donde $B_{x}$ apunta al norte geográfico, $x ; B_{y}$ al este $y ; y B_{z}$ vertical hacia abajo $z$ (figura 3.3). Entonces, las componentes de $\boldsymbol{B}$ se escriben como (3.2): 


$$
\begin{array}{r}
B_{x}=-B_{\theta}=\frac{1}{r} \frac{\partial V}{\partial \theta} \\
B_{y}=B_{\phi}=-\frac{1}{r \operatorname{sen} \theta} \frac{\partial V}{\partial \phi} \\
B_{z}=-B_{r}=\frac{\partial V}{\partial r}
\end{array}
$$

a

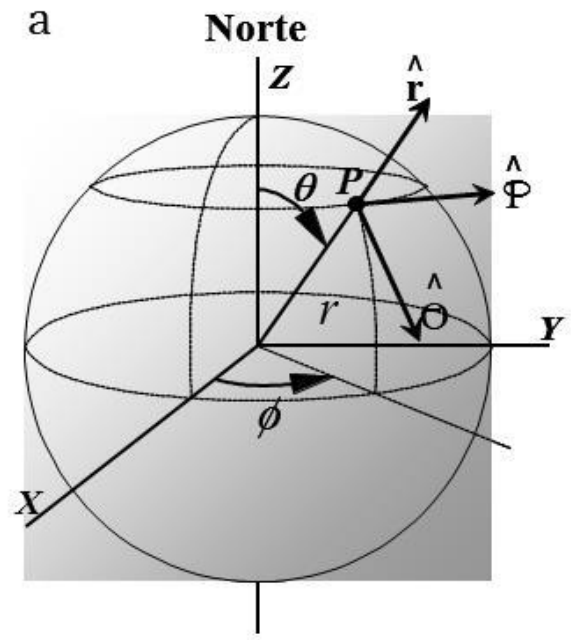

Sur $\mathrm{b}$

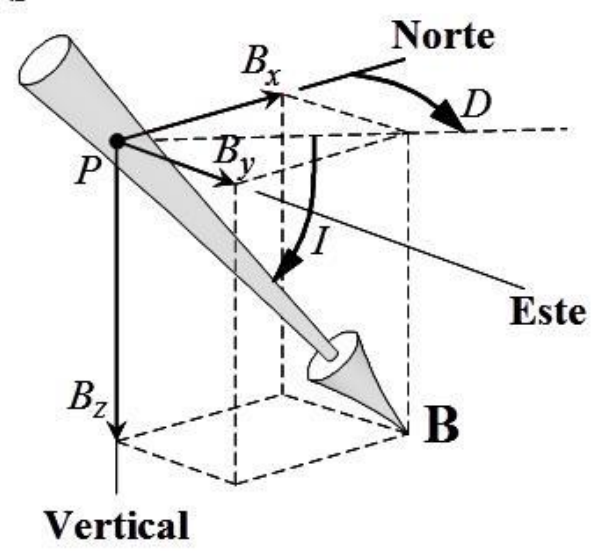

Figura 3.3. Gráfico adaptado de Blakely, 1995. a) Sistema de coordenadas esféricas. El punto $P$ está definido por las coordenadas $r, \theta$ y $\emptyset$, y un vector en el punto $P$ se describe en términos de tres vectores unitarios ortogonales: $\widehat{r}, \widehat{\Theta}, y, \widehat{\Phi}$. b) Sistema de coordenadas cartesianas del punto P. Se muestran las tres componentes del vector $B$ : $B_{\mathrm{x}}$ es positivo en dirección norte $\left(B_{\mathrm{x}}=-B_{\theta}\right), B_{\mathrm{y}}$ positivo hacia el este $\left(B_{\mathrm{y}}=B_{\emptyset}\right)$, y $B_{\mathrm{z}}$ es positivo hacia abajo $\left(B_{\mathrm{z}}=-B_{\mathrm{r}}\right)$. La inclinación $I$ es el ángulo que forma $B$ con el plano horizontal, positivo hacia abajo; la declinación $D$ es el acimut de la proyección horizontal de $B$, positivo hacia el este.

Los elementos del vector del campo geomagnético en el sistema ortogonal cartesiano, se pueden definir por las relaciones (3.3). $H$ es la intensidad de la componente horizontal, $T$ la intensidad total; e $I$ y $D$ la inclinación y declinación magnética respectivamente. $I$ es el ángulo vertical entre el vector y el plano horizontal, y $D$ es el ángulo entre el meridiano magnético y el meridiano geográfico.

$$
\begin{gathered}
H=\sqrt{B_{x}^{2}+B_{y}^{2}} \\
B=\sqrt{B_{x}^{2}+B_{y}^{2}+B_{z}^{2}}
\end{gathered}
$$




$$
\begin{gathered}
I=\arctan \frac{B_{z}}{\sqrt{B_{x}^{2}+B_{y}^{2}}} \\
D=\operatorname{arcsen} \frac{B_{y}}{\sqrt{B_{x}^{2}+B_{y}^{2}}}
\end{gathered}
$$

En principio se necesitaría un número infinito de coeficientes de Gauss para definir completamente el campo. El Campo de Referencia Geomagnético Internacional (IGRF por sus siglas en inglés) es un modelo global del campo principal interno, calculado hasta $n=10$. Este modelo se actualiza a intervalos regulares de 5 años e incluye la tasa de variación temporal de los coeficientes (variación secular) para interpolarlos. El campo magnético originado dentro de la tierra es aproximadamente dipolar: de hecho los coeficientes de primer orden del desarrollo de armónicos esféricos, es decir, $g_{1}^{0}, g_{1}^{1}$ y $h_{1}^{1}$, son mucho mayores que los subsiguientes.

Fuentes externas tales como corrientes en la ionósfera producen variaciones temporales de corto periodo en el campo geomagnético. Las de períodos de un día se conocen como variación diurna.

Los conceptos brevemente descriptos aquí intervienen en la reducción de los datos para arribar al campo de anomalías magnéticas presentes en la corteza terrestre.

\subsubsection{Procesamiento del dato aeromagnético}

A continuación se realiza una descripción de las técnicas utilizadas en la reducción de los datos provenientes de un relevamiento aeromagnético, tal como el que se realizó en el volcán Auca Mahuida.

En la primera etapa se evalúan los datos y grillas; y se remueven anomalías importantes o picos presentes en los mismos (despiking). Luego se identifican los errores.

Los errores en los datos relevados se pueden clasificar en dos tipos: error sistemático y ruido sistemático. El efecto más evidente de tales errores es un corrimiento aparente de nivel, una diferencia entre líneas paralelas vecinas de un relevamiento, conocido como error de levelling.

Las fuentes de error sistemático incluyen la variación diurna del campo magnético terrestre, rumbo (heading) del avión, variación de los instrumentos, error de retraso o lag entre el avión y el sensor, y problemas de nivelación entre las líneas de vuelo (líneas de producción) y las de control (tie lines). Los errores sistemáticos se eliminan típicamente usando correcciones conocidas (estación base diurna, lag, heading, y el IGRF).

El ruido sistemático es el error residual en los datos que permanece luego de aplicar las correcciones del error sistemático. Este ruido se debe usualmente a gradientes o 
diferencias de elevación en los datos. Para corregirlo se usan métodos de levelling convencional y levelling empírico.

Los errores magnéticos dependientes del tiempo están relacionados con la variación diurna del campo magnético terrestre. Una técnica habitual para corregirlos es medir el campo magnético en una locación terrestre fija (estación base), cercana o dentro del área del relevamiento. Esta técnica supone que las variaciones temporales del campo magnético en la estación base serán las mismas que en las posiciones por donde pasa el sensor en movimiento. Esto es aceptable para relevamientos pequeños (50-100 km), incluyendo la mayoría de los relevamientos terrestres, pero puede ser una mala suposición para relevamientos extensos en los cuales la estación base queda lejos del área cubierta. Si bien habitualmente se supone que las variaciones temporales del campo geomagnético registradas en la estación base son representativas de las mismas variaciones a lo largo de toda el area del relevamiento, tales variaciones no están totalmente sincronizadas y pueden cambiar su fase y amplitud significativamente en distancias del orden de $50 \mathrm{~km}$.

Partiendo de esta suposición, en la que la base y la aeronave registran los mismos valores del campo externo, la diferencia entre la medición en un instante de referencia dado, $t_{0}$ y otro cualquiera, será el mismo para ambos magnetómetros (el del vuelo y la estación base). Por lo tanto es válida la relación (3.4):

$$
B\left(\mathrm{t}, \mathrm{x}_{\text {base }}\right)-B\left(\mathrm{t}_{0}, \mathrm{x}_{\text {base }}\right)=B\left(\mathrm{t}, \mathrm{x}_{\mathrm{r}}\right)-B\left(\mathrm{t}_{0}, \mathrm{x}_{\mathrm{r}}\right)
$$

donde:

$B\left(t, x_{\text {base }}\right) \quad$ Es el valor del módulo del campo magnético registrado en el magnetómetro base durante la ejecución del relevamiento aeromagnético sincronizado con el tiempo del magnetómetro en el avión.

$B\left(\mathrm{t}_{0}, \mathrm{x}_{\text {base }}\right) \quad$ Es el valor del campo magnético de la estación base en el instante de referencia.

$B\left(\mathrm{t}, \mathrm{x}_{\mathrm{r}}\right) \quad$ Es el valor del campo magnético registrado durante el relevamiento aeromagnético.

$B\left(\mathrm{t}_{0}, \mathrm{x}_{\mathrm{r}}\right) \quad$ Es el valor del campo magnético registrado durante el relevamiento aeromagnético en el tiempo de referencia.

Con esta relación es posible realizar la reducción temporal de las mediciones aeromagnéticas respecto a un determinado instante en el tiempo representada por el valor $B\left(\mathrm{t}, \mathrm{x}_{\mathrm{r}}\right)$ en la ecuación (3.2). Este procedimiento no implica la remoción completa de las variaciones del campo externo sino que pretende reducirlas a un valor aproximadamente constante para todo el relevamiento. De este modo las variaciones del campo externo no afectan el cálculo de la anomalía.

El error de lag (retraso) resulta cuando el sistema de posicionamiento del 
relevamiento es significativamente distinto de la localización física del sensor (como en los relevamientos en configuración towed bird). Esta corrección ajusta la línea de tiempo de las lecturas para reproducir los datos de posicionamiento.

Los errores de heading (rumbo) se relacionan con el campo magnético de la plataforma de medición, el cual varía con la dirección del relevamiento, dando origen a corrimientos sistemáticos en los datos.

Luego de la reducción temporal pueden quedar en los datos diferencias de nivel en las medidas del campo magnético a lo largo de los perfiles de vuelo. Esto puede ser causado por la incompleta remoción de las variaciones del campo externo, de los errores del GPS en el posicionamiento de las medidas o de los errores del láser del altímetro en la estimación de las cotas. Estos errores se remueven con la técnica de levelling que utiliza las mediciones de los perfiles de control (tie line). Las tie lines son líneas de control que normalmente se recorren perpendiculares a las líneas regulares del relevamiento. Si los datos no contienen error de levelling, entonces las tie lines y las líneas del relevamiento tendrán los mismos valores en los puntos donde se cruzan. Si los valores son distintos debe aplicarse corrección de levelling.

Las correcciones de levelling se pueden aplicar de modo convencional o empírico. El enfoque convencional requiere evaluar las líneas de control y las líneas regulares juntas. Primero se ajustan todas las tie lines, y luego las regulares se ajustan para que coincidan con aquéllas. Los ajustes consisten en aplicar un único tipo de tendencia a todas las líneas, regulares y tie lines (levelling simple) o bien aplicar tendencias individuales a líneas o grupos de líneas (levelling detallado).

La reducción de los datos continúa con la remoción de la contribución debida al campo de origen nuclear. Esta componente se determina con el modelo de referencia IGRF, que es una función de la latitud y longitud, de la cota y el tiempo. El campo de la anomalía de la corteza finalmente se calcula como la diferencia entre los datos una vez aplicado el levelling y el valor del modelo utilizado para el campo principal en cada posición de medición. Los valores obtenidos se interpolan, por ejemplo, con el algoritmo de mínima curvatura, considerado el mejor método para la interpolación de anomalías en los datos de campos potenciales (De Ritis, 2004).

Cuando la remoción de las variaciones del campo externo no es completa, queda un ruido residual en la anomalía obtenida. Para eliminar este residuo se utiliza la técnica de microlevelling. El levelling empírico o microlevelling es una técnica de filtrado matemático que se le aplica a los datos interpolados para eliminar ruido residual asociado a las líneas de vuelo. Estos son filtros bidimensionales de "decorrugamiento" que se aplican sobre la matriz de los datos transformados al dominio de la frecuencia, y se usan para identificar pequeñas variaciones entre líneas. Es necesario asegurarse de que el ruido que se está tratando de eliminar no esté presente en la señal natural en la misma dirección de filtrado. En este sentido, los parámetros que controlan la intensidad del filtro representan un aspecto crítico. La diferencia de las matrices de los datos antes y después del microlevelling proporciona la matriz de errores eliminados. La corrección de microlevelling se obtiene sustrayendo de la matriz del campo de anomalía la matriz de los errores obtenidos (De Ritis, 2004). 
Por lo tanto, el procesamiento de datos del VAM siguió los pasos antes mencionados, que se pueden resumir en la forma siguiente: eliminación de ruidos y picos espúreos mediante filtrado, técnica conocida como despiking. Los datos fueron correlacionados con la posición dada por GPS. A lo largo de cada perfil, se hicieron las correcciones para la remoción de la contribución debida al campo de origen nuclear, en las que se usó el IGRF para la fecha 2001.2056. Posteriormente a que los datos fueran corregidos por el IGRF, se aplicó la corrección por variación diurna del campo magnético a partir del registro de un magnetómetro base ubicado en el aeropuerto de Rincón de los Sauces (lugar desde donde partió el helicóptero a $30 \mathrm{~km}$ en dirección norte del VAM). Luego de que se volaran todas las líneas en la grilla se hizo un análisis de las intersecciones (levelling) y se volvieron a volar todas las líneas "ruidosas" antes de que el helicóptero abandonara el área.

La secuencia del proceso se podría resumir como:

1- Evaluación de datos y grillas Identificación de errores sistemáticos Identificación de ruido sistemático

2- Aplicación de correcciones sistemáticas

Corrección de estación base

Corrección de retardo (lag)

Corrección de rumbo (heading)

Corrección IGRF

3- Aplicación de levelling convencional (tie line)

(Simple o detallado)

4- Aplicación de levelling empírico (microlevelling).

\subsubsection{Procesamiento del dato gravimétrico}

La gravedad observada o medida, $g_{o b s,}$ en un relevamiento móvil está formada por una serie de contribuciones de las cuales sólo nos interesa la producida por las fuentes someras. Esto se resume como (Blakely, 1995):

Gravedad observada $=$

(1) efecto de la aceleración plataforma móvil (Eötvös)

+ (2) atracción del conjunto de masas terrestres por debajo del nivel de referencia (nivel medio del mar)

+ (3) efecto de la elevación sobre el nivel del mar (aire libre)

+ (4) efecto de la masa topográfica sobre el nivel del mar (Bouguer y Topográfica)

+ (5) variaciones dependientes del tiempo (marea) y deriva del instrumental

+ (6) efecto de las masas que soportan carga topográfica (Isostasia)

+ (7) efecto de variaciones de densidad de la corteza y manto superior (geología) 
En este estudio el objetivo consiste en aislar la última cantidad (7) de la expresión anterior, es decir, el efecto de las variaciones de densidad de la corteza y manto superior. Para lo cual debe aislarse la anomalía residual mediante la aplicación de filtros que serán descriptos en el Capítulo VI.

(1) El tratamiento de los datos gravimétricos comienza con la corrección de los efectos dinámicos. En el caso que nos ocupa, los datos de gravedad fueron medidos desde una plataforma que al estar en movimiento, hace que actúe la fuerza centrífuga. Esto hace que se requiera aplicar la corrección de Eötvös, conociendo la posición horizontal y vertical del helicóptero. Para esta corrección se usó la siguiente ecuación (3.5) (Nettleton, 1940):

$$
g_{e t v}=\frac{(a+H)}{a^{2}}\left(2 a \omega v \cos \lambda \operatorname{sen} \alpha+v^{2}\right)
$$

donde $g_{e t v}$ es la corrección de Eötvös, $v$ la velocidad del helicóptero, $H$ la elevación sobre el nivel del mar, $a$ el radio terrestre, $\alpha$ el heading, $\lambda$ la latitud y $\omega$ la velocidad angular de rotación de la Tierra.

A modo de ejemplo se muestran en la figura 3.4 los valores de la corrección de Eötvös como función del rumbo (dirección respecto al norte, en grados) manteniendo constantes los parámetros promedio del relevamiento aéreo del VAM (altitud de 2830 m, velocidad de 92.6 $\mathrm{km} / \mathrm{h}$, latitud $37.4^{\circ} \mathrm{S}$ ).

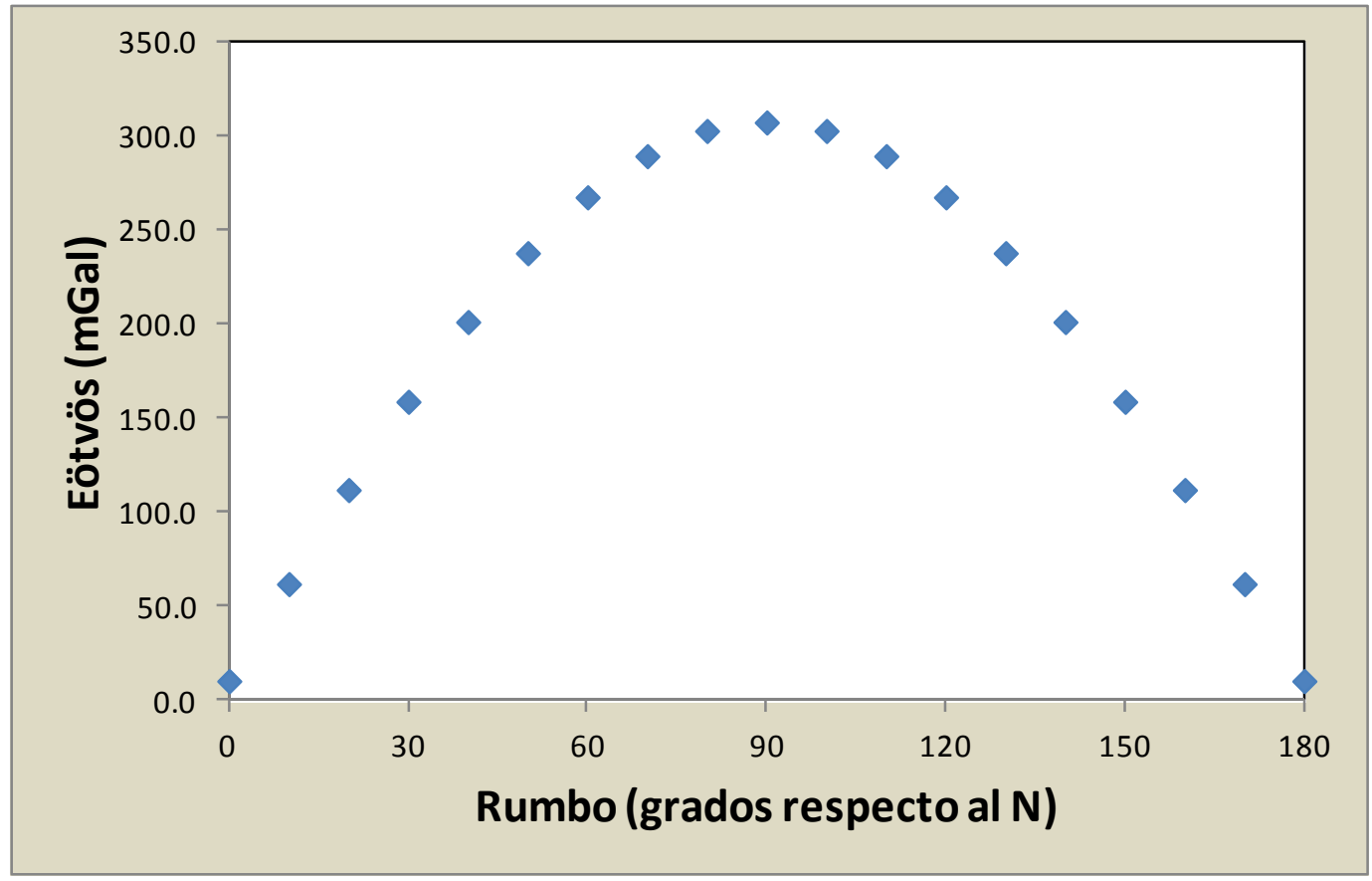

Figura 3.4: Corrección de Eötvös como función del rumbo con los parámetros promedio del relevamiento. 
Las aceleraciones verticales se calculan de los valores de altitud derivados de los sensores de presión bidireccionales y de la información de posición vertical obtenida del GPS. Las altitudes derivadas de la presión tienen una deriva debida a pendientes barométricas lineales y no lineales que se remueven antes del cómputo. La siguiente ecuación (3.6) se usa para el cálculo de las aceleraciones verticales:

$$
A=\frac{\left(E_{3}-E_{2}\right)-\left(E_{2}-E_{1}\right)}{t^{2}}
$$

donde $A$ es la aceleración vertical, $E_{1}$ elevación en el punto $1, E_{2}$ elevación en el punto $2, E_{3}$ elevación en el punto $3, t$ diferencia temporal o frecuencia de muestreo.

La reducción de los datos gravimétricos se realizó con el fin de obtener las anomalías gravimétricas, es decir, la diferencia entre el valor de gravedad observada y corregida ( $g_{\text {cobs }}$ ) en un punto de la superficie y la gravedad teórica $(\gamma)$, obtenida de un modelo de referencia. Esto es, $\Delta \mathrm{g}=\mathrm{g}_{\mathrm{cobs}}-\gamma$.

(2) Debido a la complejidad de las variaciones internas de densidad, se acostumbra referenciar el geoide a una superficie más simple y suave. En virtud de un acuerdo internacional, esa superficie equipotencial es la superficie de un elipsoide que limitaría una Tierra rotante y de densidad uniforme. Las diferencias de altitud entre ese elipsoide de referencia y el geoide son generalmente menores a $90 \mathrm{~m}$ y reflejan variaciones laterales de densidad uniforme así como su forma.

Para los datos del VAM se utilizó la Fórmula Internacional de gravedad para el Sistema de Referencia Geodésico de 1967 (GRS67), expresada como (Blakely, 1995):

$$
\gamma=9.78031846\left(1+0.0053024 \sin ^{2} \lambda-0.0000058 \sin ^{2} 2 \lambda\right)
$$

donde $\gamma$ es la gravedad teórica en gales y $\lambda$ es la latitud.

(3) La corrección de aire libre se aplica a mediciones gravimétricas realizadas por encima del nivel del mar, y tiene en cuenta la diferencia en la atracción gravitatoria debida sólo a la altura (distancia del punto de medición al centro de la Tierra), sin tomar en cuenta las masas existentes. Si $g(r)$ representa la atracción gravitatoria del elipsoide de referencia, entonces el valor de la gravedad a una altura $h$ por encima del nivel del mar, pequeña comparada con el radio de la Tierra, está dada por el desarrollo en serie de Taylor:

$$
g(r+h)=g(r)+h \frac{\partial}{\partial r} g(r)+\cdots
$$

Conservando hasta el primer orden, reordenando y suponiendo una Tierra esférica 
$\left(g(r)=-G M / r^{2}\right)$ resulta:

$$
g(r)=g(r+h)-\frac{2 g(r)}{r} h
$$

donde $\mathrm{G}$ es la constante de gravitación universal y es igual a $6.67 \times 10^{-11} \mathrm{~m}^{3} \mathrm{~kg}^{-1} \mathrm{~s}^{-2}$ en unidades SI y 6.67 x $10^{-8} \mathrm{~cm}^{3} \mathrm{~g}^{-1} \mathrm{~s}^{-2}$ en unidades cgs.

El último término es la corrección de aire libre $g_{A L}$. Usando los valores de $g$ y $r$ a nivel del mar resulta ( $g_{A L}$ en gal y $h$ en cm, o bien $g_{A L}$ en $\mathrm{ms}^{-2}$ y $h$ en m):

$$
g_{A L}=-0.3086 \times 10^{-5} h
$$

$h$ es la altura de observación aérea sobre el nivel del mar.

Por lo tanto, la anomalía de aire libre está dada por:

$$
A A L=g_{o b s}-g_{A L} \pm g_{e t v}-\gamma
$$

donde $g_{\text {etv }}$ es la corrección por el efecto de Eötvös.

(4) Tanto la gravedad teórica como la corrección de aire libre ignoran la masa existente entre el nivel de la medición y el nivel del mar. Esta masa adicional es tomada en cuenta en la corrección de Bouguer. La corrección de Bouguer simple aproxima toda la masa sobre el nivel del mar con una capa homogénea infinita de espesor igual a la altitud del punto de observación desde el nivel del mar con densidad $\rho$. La atracción debida a esa placa da lugar a una aceleración:

$$
g_{\text {placa }}=2 \pi G \rho h
$$

donde $h$ es el espesor de la placa. Usando un valor típico de densidad de la corteza de 2.67 $\mathrm{g} / \mathrm{cm}^{3}$, la corrección resulta:

$$
g_{\text {placa }}=0.1119 \times 10^{-5} h
$$

$g_{\text {placa }}$ en gal y $h$ en cm, o bien $g_{\text {placa }}$ en $\mathrm{ms}^{-2}$ y $h$ en m. Como se verá en el Capítulo VI para los datos del VAM se usó un valor de densidad distinto tanto para esta corrección como para la topográfica.

La anomalía simple de Bouguer (ABS) ignora la forma de la topografía: masas por encima del nivel de observación tales como montañas no son tenidas en cuenta en la placa y 
valles por debajo de esa elevación forman cavidades dentro de la placa. Por lo tanto, la corrección simple de Bouguer tiende a sobrecompensar las medidas efectuadas cerca de la superficie. De moderado a alto relieve topográfico la aproximación de una capa uniforme no es válida y se hace necesario introducir una corrección por topografía, $g_{\mathrm{t}}$ (Blakeley, 1995). Las correcciones de terreno en gravimetría aerotransportada consisten en el efecto gravitacional teórico producido por una capa de roca rellena desde el nivel del aparato aéreo hasta el nivel del mar, menos la topografía. Entonces, la anomalía de Bouguer completa (ABC) para observaciones aéreas resulta (Hinze et al., 2013):

$$
A B C=g_{o b s}-g_{A L}-g_{\text {placa }} \pm g_{t} \pm g_{e t v}-\gamma
$$

Reemplazando la ecuación (3.10) y (3.11) en (3.14) se obtiene:

$$
A B C=g_{o b s}+0.3086 h-2 \pi G \rho\left(h-h_{G S}\right) \pm g_{t} \pm g_{e t v}-\gamma
$$

donde $h_{G S}$ es la altura de la observación aérea sobre la topografía.

(5) Las mareas terrestres causadas por el sol y la luna son de amplitud suficiente para ser detectadas por los gravímetros como una gravedad variable en el tiempo. El efecto es mayor a bajas latitudes y tiene una componente periódica intensa de período aproximado 12 horas. El efecto de marea nunca excede $3 \times 10^{-6} \mathrm{~m} \mathrm{~s}^{-2}$ ( $\left.0.3 \mathrm{mGal}\right)$, una cantidad pequeña comparada con otras correcciones a la gravedad observada. Sin embargo, los efectos de mareas deben ser tenidos en cuenta en relevamientos de alta precisión. Para relevamientos menos precisos puede resultar adecuado suponer que el efecto de marea es lineal en períodos de algunas horas y removerlo junto con otras correcciones temporales (por ejemplo, la deriva del instrumento).

(6) Se llama isostasia a la compensación de un exceso (aparente) de masa de un rasgo topográfico por una estructura de menor densidad en el subsuelo. Estructuras tales como cordilleras o continentes, de los cuales se esperaría una atracción gravitatoria lateral neta, no la evidencian en la magnitud esperada debido a la compensación ejercida por la "deficiencia" de masa en el volumen de la estructura sub-superficial. Las dos hipótesis principales de estas anomalías son debidas a Airy y a Pratt. La teoría de Airy considera que las masas de compensación son ondulaciones de la interfase corteza-manto, como en el caso de las raíces debajo de montañas (las raíces son de material de corteza de baja densidad, penetrando en el manto de mayor densidad). En cambio, Pratt propuso que la densidad varía lateralmente en la corteza de modo que toda sección vertical de corteza tiene una masa constante.

En el presente caso de estudio esta corrección no fue aplicada por las dimensiones de escala local del relevamiento. 


\subsection{Datos regionales}

Los datos aéreos magnéticos y gravimétricos del relevamiento de alta resolución del VAM están comprendidos dentro del plateau volcánico. Para el análisis de los campos de anomalías, es importante contar con información que se extienda fuera del área de interés la cual posibilitaría incluir efectos regionales en la interpretación. De hecho, para los fines de la interpretación es necesario evaluar cualitativa y (siempre que sea posible) cuantitativamente el modo en que las longitudes de onda regionales afectan a las longitudes de onda contenidas en la medición.

\subsubsection{Magnéticos}

Se incorporaron a la base de datos de la tesis registros aeromagnéticos regionales de baja resolución del bloque "Cuenca Neuquina 1968" adquiridos por la Gerencia de Exploración YPF en 1968. Esto implica que los datos regionales tienen menor calidad que los medidos en el VAM en 2001. En el año 2007, el Servicio Geológico Minero Argentino (Ferpozzi et al., 2007) digitalizó, reprocesó y editó los datos regionales analógicos originales. El relevamiento se subdividió en tres bloques con alturas de vuelo diferentes (1000, 2500 y $4000 \mathrm{msnm}$ ) de acuerdo a la topografía. Se compone de líneas de vuelo de orientación N10 distanciadas $10 \mathrm{~km}$, cruzadas por líneas de orientación $\mathrm{N} 100^{\circ}$ separadas $30 \mathrm{~km}$ (tie lines) y varias líneas diagonales de disposición irregular (figura 3.5). Se empleó un magnetómetro de precesión protónica, con intervalo de muestreo de 1 minuto equivalente a una distancia entre puntos de 2 a $3 \mathrm{~km}$. Los centros de operaciones y de control de variación magnética diurna fueron San Rafael, Malargüe y Rincón del Sauces. Para la integración digital de los sectores volados a diferentes alturas, se realizó una continuación ascendente hasta $2000 \mathrm{~m}$ en aquellos sectores volados a alturas inferiores. Para la remoción de la componente nuclear se usó el modelo del IGRF correspondiente a la fecha 1968.5.

Desafortunadamente, estos datos no cubren el edificio central del VAM, de modo que sólo serán usados para un análisis cualitativo a fines de caracterizar los rasgos magnéticos en las longitudes de onda más larga que afectan a los límites de la anomalía del campo magnético total de 2001 (figura 3.5). 


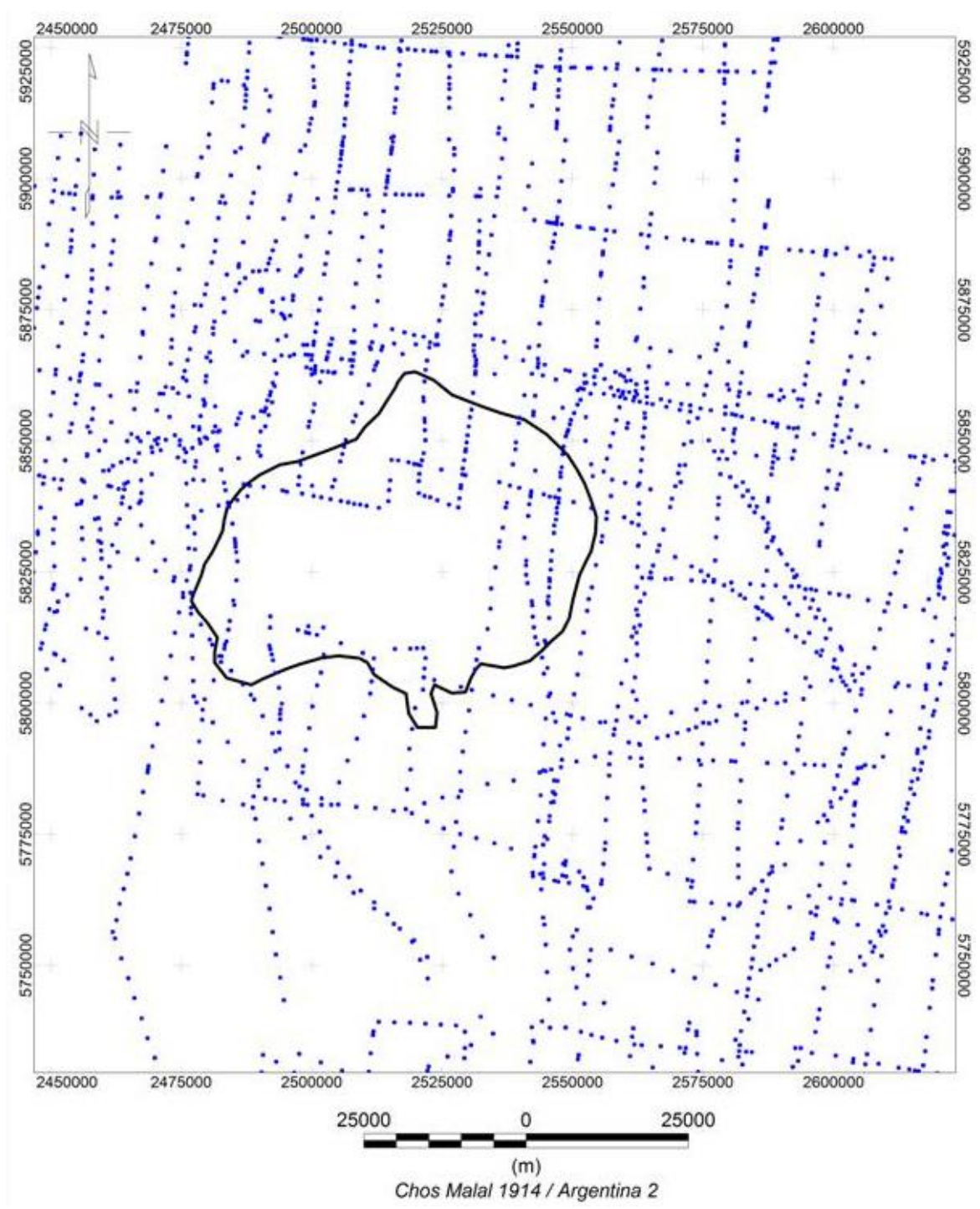

Figura 3.5. Datos aeromagnéticos regionales. El patrón de líneas de vuelo se representa en puntos de color azul. Se observa que los datos no cubren completamente el plateau volcánico del volcán Auca Mahuida.

\subsubsection{Gravimétricos}

Los datos gravimétricos de la Cuenca Neuquina existentes en la base de datos de YPF S. A., provienen de planos en papel de la propia YPF S. A. y del Instituto Geográfico Nacional (IGN).

Las estaciones de gravedad levantadas entre los años 1966 y 1975 por YPF S. A. se conservan en cartas a escala 1:50.000. En ellas se incluye la localización de cada estación y su valor de Anomalía de Bouguer ( $\mathrm{AB}$ ) relativa en unidades gravimétricas ( $\mathrm{UG}=0.1 \mathrm{mGal}$ ) calculada para un valor promedio de densidad superficial de $1.97 \mathrm{~g} / \mathrm{cm}^{3}$. Los planos no poseen datos de cota, por lo que se usó una constante altimétrica de $2.26 \mathrm{UG} / \mathrm{m}$. El total de estaciones gravimétricas recopiladas fue de 28511. Los valores de gravedad medidos son relativos a una estación de referencia ya que no se medían valores absolutos de gravedad 
(comunicación oral; no se conoce informe escrito). La base de datos provista por el IGN contiene un total de 3735 estaciones con coordenadas X e Y, cota y gravedad absoluta en mGal (Profeta, 2000).

Todos los levantamientos gravimétricos terrestres realizados en la cuenca fueron digitalizados por Repsol-YPF a finales de la década de 1990 (Profeta, 2000), donde se incluyeron las coordenadas de posición (norte y este) en el sistema Gauss-Kruger y el valor de AB. Se logró recuperar un total de 30854 estaciones. De acuerdo a lo que se observa en la figura 3.6, se podría inferir que la distribución regular de los puntos en algunas zonas es producto de la interpolación de la grilla. Adicionalmente, se digitalizaron las alturas provenientes de cada estación gravimétrica de las cartas topográficas de la República Argentina y del aporte de elevaciones de líneas sísmicas, con lo que se pudieron obtener 59935 observaciones altimétricas. Con estos valores de altura se originó un mapa topográfico.

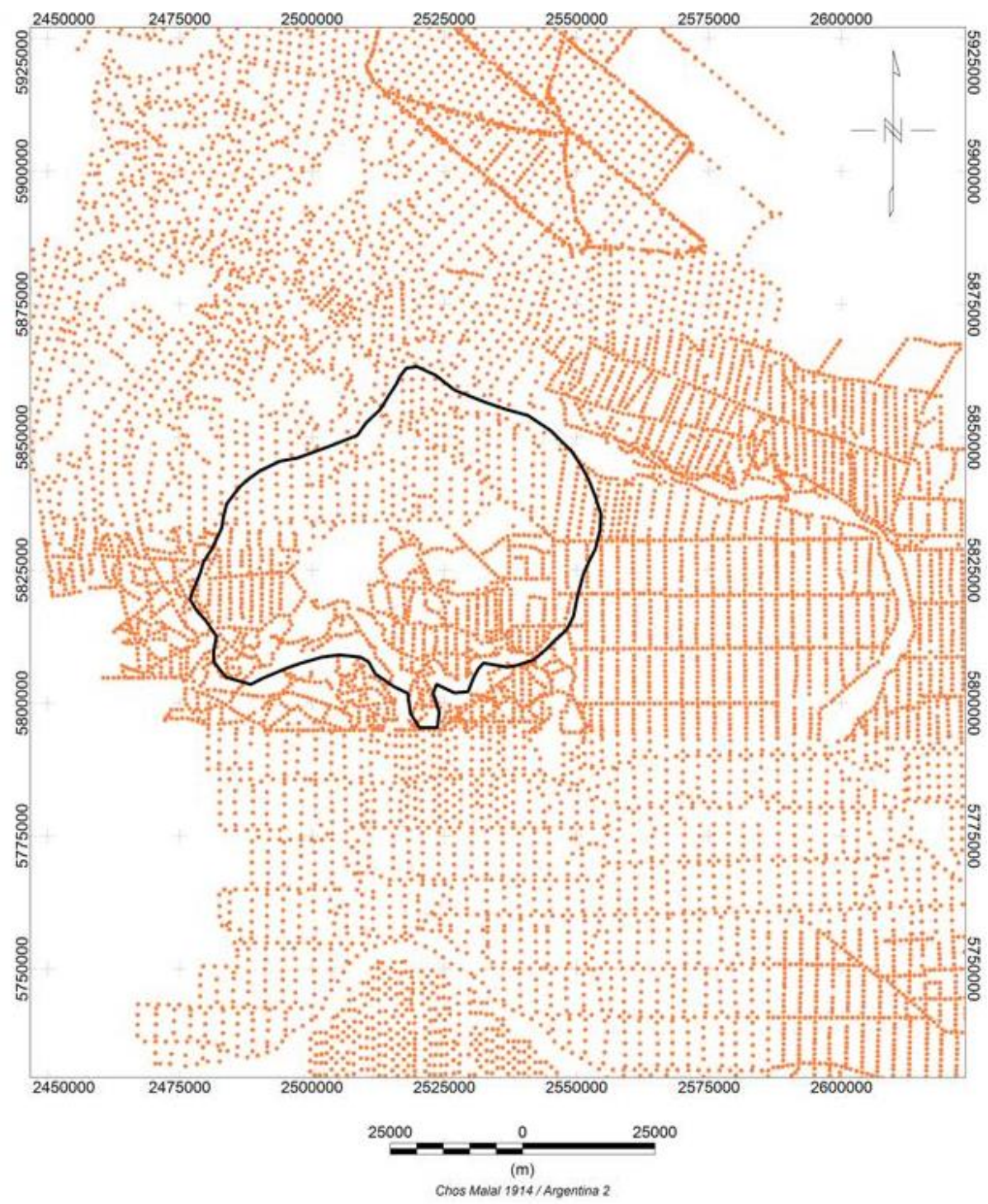

Figura 3.6. Datos gravimétricos regionales adquiridos por YPF. 


\subsection{Datos Sísmicos}

Se dispone de líneas sísmicas 2D en el dominio de tiempo en el área del VAM (figura 3.7). En la zona cercana al cráter central del volcán, la información sísmica no tiene buena relación señal-ruido debido al espesor del manto lávico superficial con alta impedancia acústica (los espesores de roca volcánica varían entre 100 y 400 m). También se cuenta con un relevamiento sísmico 3D, resultado de la unión de tres cubos migrados pre-apilado en tiempo, que cubre un área de aproximadamente $180 \mathrm{~km}^{2}$ (figura 3.8). El reprocesamiento estuvo a cargo de la compañía Western Geco en el periodo 2003-2004 y no cubre la zona del edificio central del volcán. El datum final (plano de referencia) es de $2000 \mathrm{~m}$.

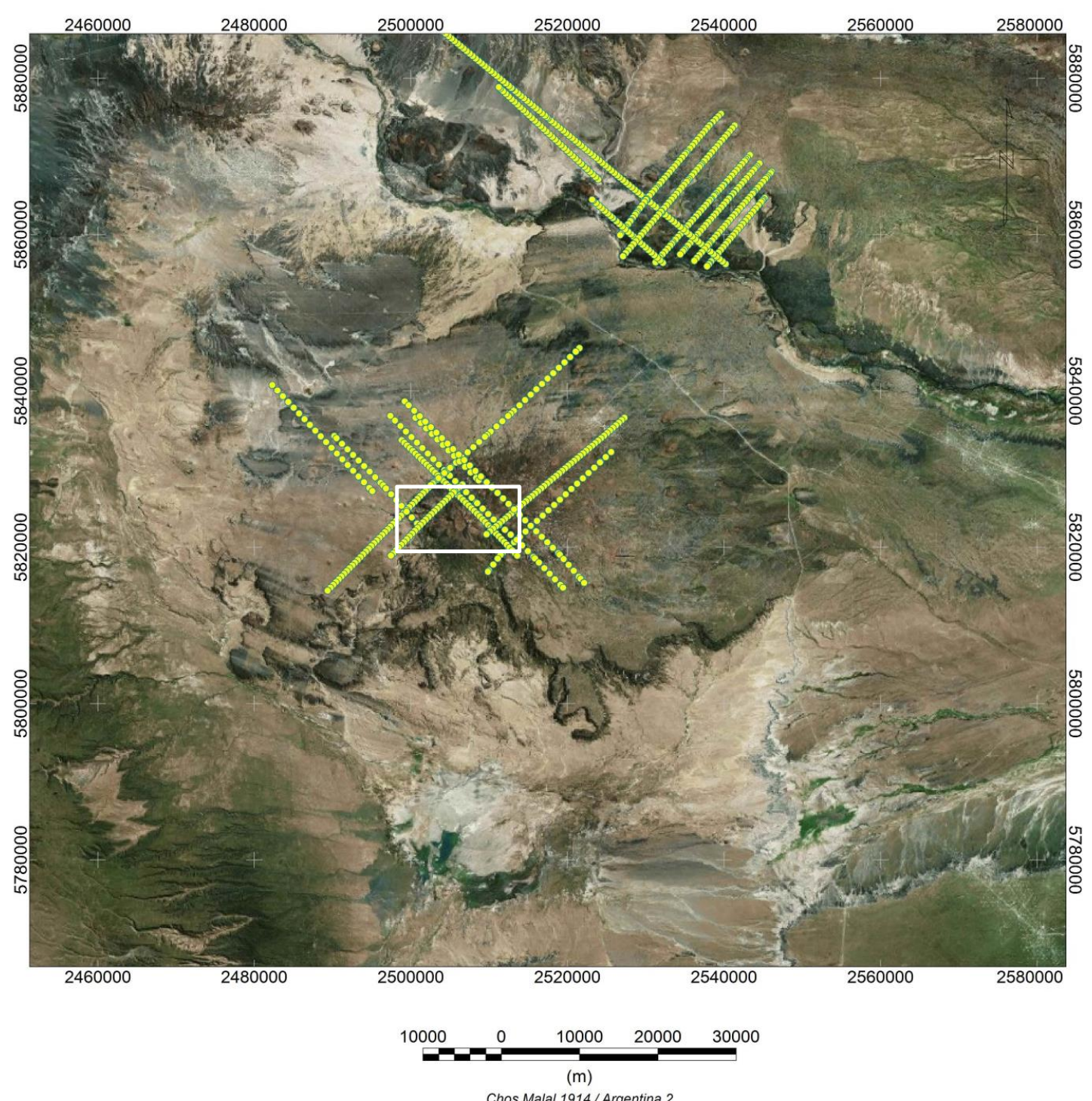

Figura 3.7. Líneas sísmicas 2D reprocesadas (color amarillo) sobre imagen satelital. El rectángulo blanco indica la ausencia de relevamiento sísmico 3D. 


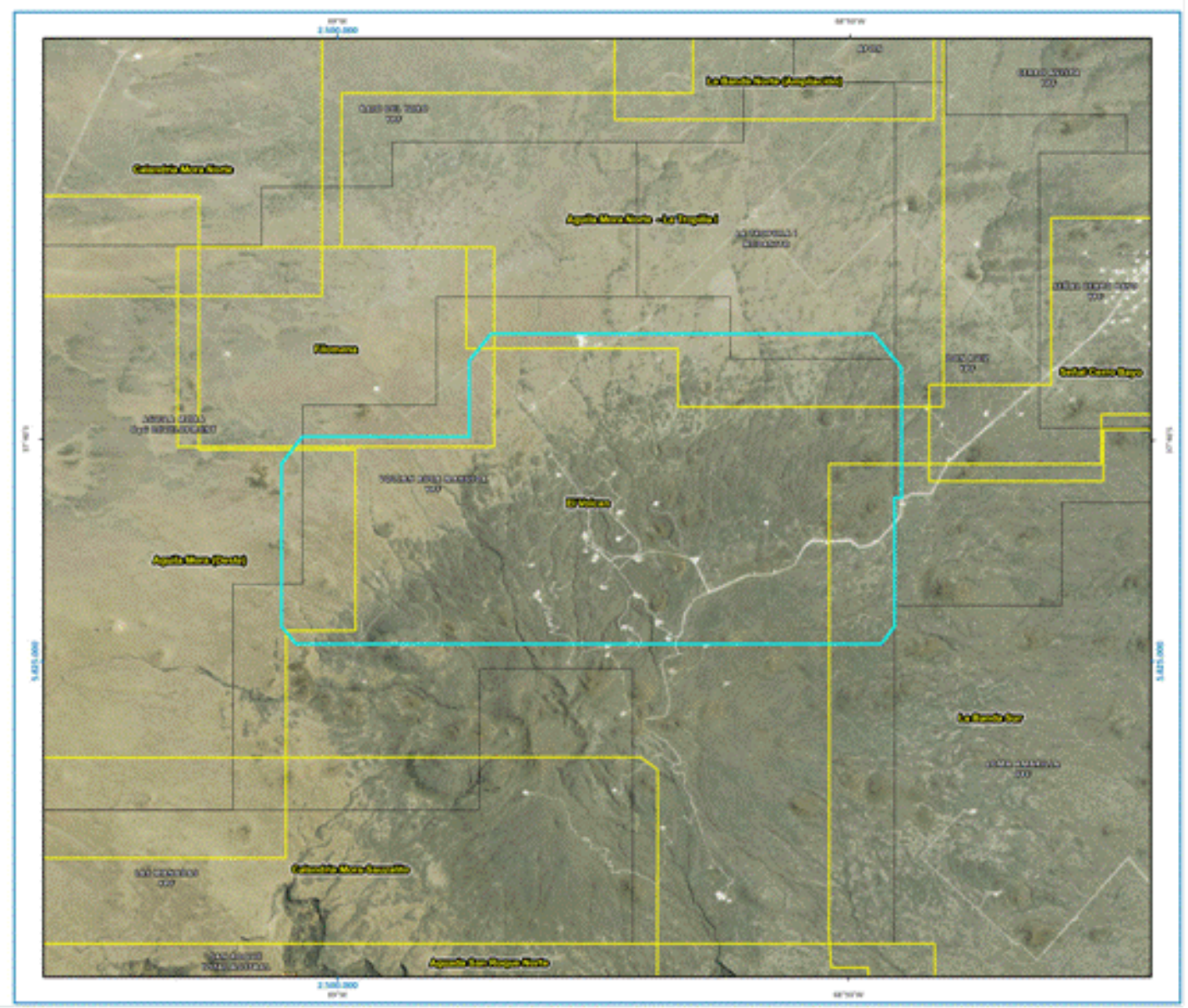

Figura 3.8. Unión de los tres relevamientos reprocesados para formar el cubo El Volcán 3D (polígono celeste).

\subsection{Datos de pozo}

Se incorporó información de pozos para ayudar a la interpretación de los datos magnéticos. Para ello se construyó una base de datos de la estratigrafía y litología de 18 pozos (tabla 3.1) que atraviesan cuerpos intrusivos, ya que son los que interesan al dato magnético. En la figura 3.9 se grafica la ubicación de estos pozos. Se incluyó además, información de las distintas formaciones geológicas atravesadas, con sus topes y espesores, y una breve descripción litológica de cada formación (Apéndice A). No todos los pozos poseen información completa de la estratigrafía. Las profundidades máximas no alcanzan los $4 \mathrm{~km}$ (medido desde el terreno), involucrando la parte superior a media de la cobertura sedimentaria. La mayoría de los pozos cuentan con perfiles sónico, densidad, rayos gamma (GR) y potencial espontáneo (SP), los cuales permiten identificar los topes y bases de las formaciones y de los intrusivos, generalmente filones capa. Además, los valores de densidad de los registros de pozo para cada formación, permiten obtener un valor promedio para la reducción de los datos gravimetricos, i. e., corrección de Bouguer y topográfica, como se verá en el capítulo VI. 
Tabla 3.1. Pozos que atraviesan cuerpos volcánicos.

\begin{tabular}{|ccc|}
\hline POZO & $X$ & $Y$ \\
\hline VAM.X-1 & 2505664.99 & 5827112.84 \\
VAM.x-2 & 2510198.00 & 5828105.00 \\
VAM. $x-3$ & 2507062.00 & 5828818.00 \\
VAM-7 & 2507352.90 & 5828241.90 \\
VAM.e-9 & 2511110.00 & 5824528.00 \\
VAM.a-10 & 2508587.63 & 5828075.57 \\
VAM-15d & 2509112.33 & 5826250.76 \\
VAM-28d & 2507692.39 & 5825594.09 \\
VAM.a-33 & 2509079.96 & 5829767.96 \\
AgL.x-1 & 2516884.00 & 5832554.00 \\
CEI.x-1 & 2531892.00 & 5824686.00 \\
CLB.x-1 & 2516240.00 & 5838327.00 \\
EV.x-2 & 2509144.00 & 5833918.00 \\
Lan.x-1 & 2510960.00 & 5818340.00 \\
LPE.x-2 & 2531150.00 & 5819800.00 \\
LRb.x-1 & 2500719.00 & 5824552.00 \\
RiAI-3 & 2505357.50 & 5821364.48 \\
SAM. $x-1$ & 2490820.00 & 5835545.00 \\
\hline
\end{tabular}

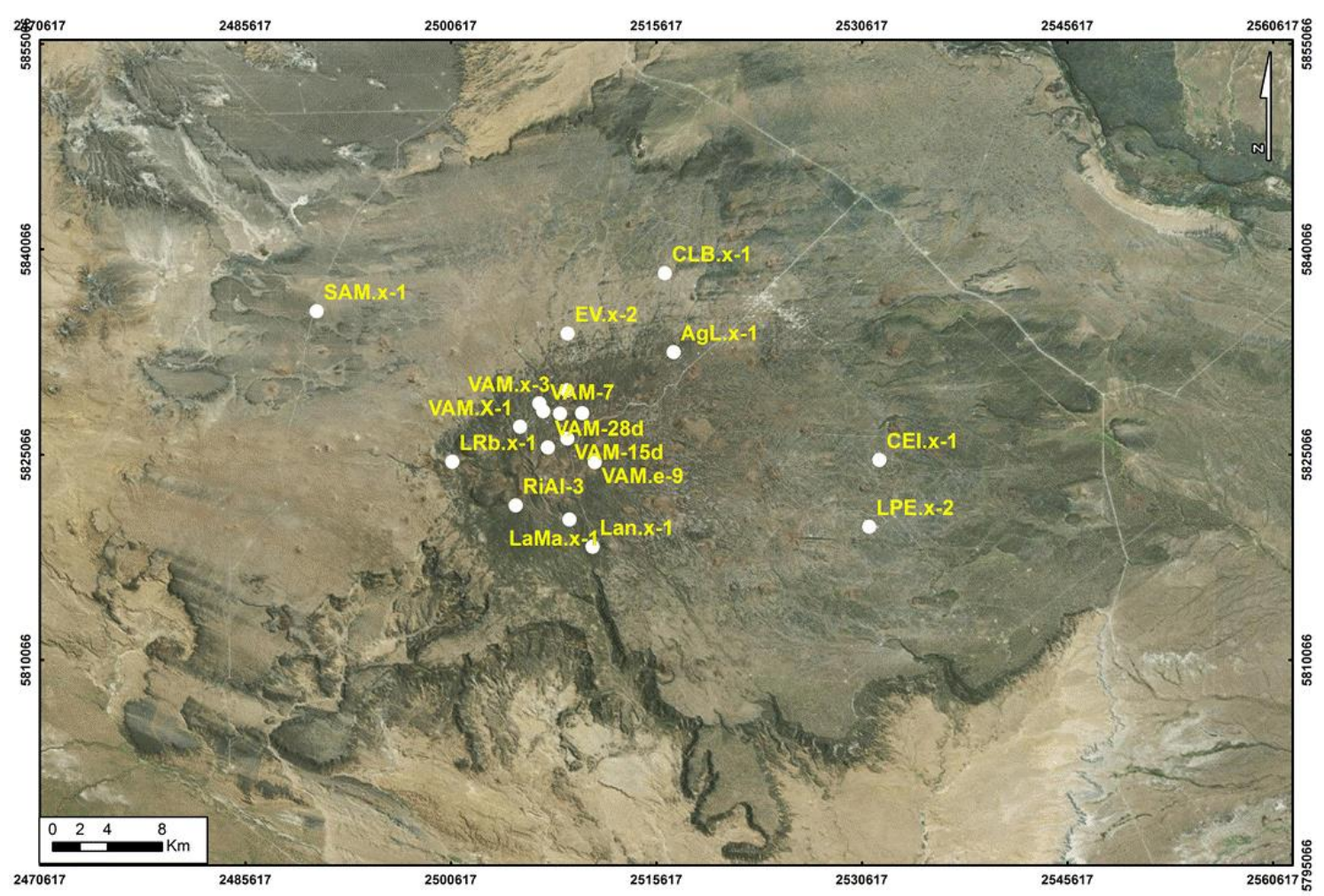

Figura 3.9. Ubicación de los pozos analizados que atraviesan cuerpos intrusivos. 
En algunos de los pozos contenidos en esta base de datos se extrajeron coronas que atraviesan rocas sedimentarias e intrusivos volcánicos. Excepto los cuerpos volcánicos intruidos, todas las formaciones perforadas son transparentes al método magnético.

\subsubsection{Espesores de basaltos}

De acuerdo con lo mencionado anteriormente, la cobertura lávica tiene un espesor considerable que alcanza los $400 \mathrm{~m}$ cerca del conducto central del volcán y va disminuyendo hacia la periferia.

Con datos provenientes de 19 perforaciones encontradas en los legajos de pozo (Longo et al., 2008) se construyó una tabla (tabla 3.2) con los espesores de las rocas volcánicas atravesadas en el sector norte del volcán. En la figura 3.10 se ubican los 19 pozos con sus respectivos espesores.

Tabla 3.2. Espesores de basalto extraídos de los legajos de pozo. X e Y son las coordenadas planas Gauss Kruger faja 2 en el sistema geodésico Chos Malal, $Z$ las cotas de superficie y la última columna los espesores de los basaltos de superficie para cada pozo.

\begin{tabular}{|ccccc|}
\hline POZO & $\mathrm{X}$ & $\mathrm{Y}$ & $\mathrm{Z}(\mathrm{m})$ & ESPESOR $(\mathrm{m})$ \\
\hline CLBL.x-1 & 2516240.00 & 5838326.93 & 1017 & 100 \\
EAj.x-1 & 2526781.00 & 5819054.00 & 1039 & 35 \\
EV.x-1 & 2505557.00 & 5832517.00 & 1250 & 110 \\
EV.x-2 & 2509144.00 & 5833917.89 & 1227 & 180 \\
F.x-1 & 2496777.00 & 5834101.00 & 1087 & 80 \\
LAn.x-1 & 2510960.03 & 5818339.91 & 1458 & 214 \\
LRb.x-1 & 5824552.00 & 2500719.00 & 1557 & 410 \\
PDCh.x-1 & 2521031.00 & 5832578.00 & 1102 & 137 \\
SCB.e-2 & 2525029.00 & 5837104.00 & 920 & 42 \\
SCBS.x-1 & 2526833.00 & 5826522.00 & 957 & 120 \\
VAM.x-1 & 2505664.99 & 5827112.84 & 1676 & 352 \\
VAM-10 & 2508587.00 & 5828075.00 & 1537 & 298 \\
VAM-11 & 2509144.00 & 5826273.00 & 1701 & 385 \\
VAM-12 & 2507868.48 & 5830598.79 & 1367 & 130 \\
VAM-3 & 2507062.00 & 5828817.89 & 1467 & 310 \\
VAM-4 & 2507310.00 & 5828085.00 & 1530 & 253 \\
VAM-5 & 2508571.00 & 5827044.00 & 1583 & 290 \\
VAM-6 & 2507144.00 & 5829648.89 & 1400 & 260 \\
VAM-8 & 2507599.44 & 5827293.92 & 1598 & 325 \\
\hline
\end{tabular}




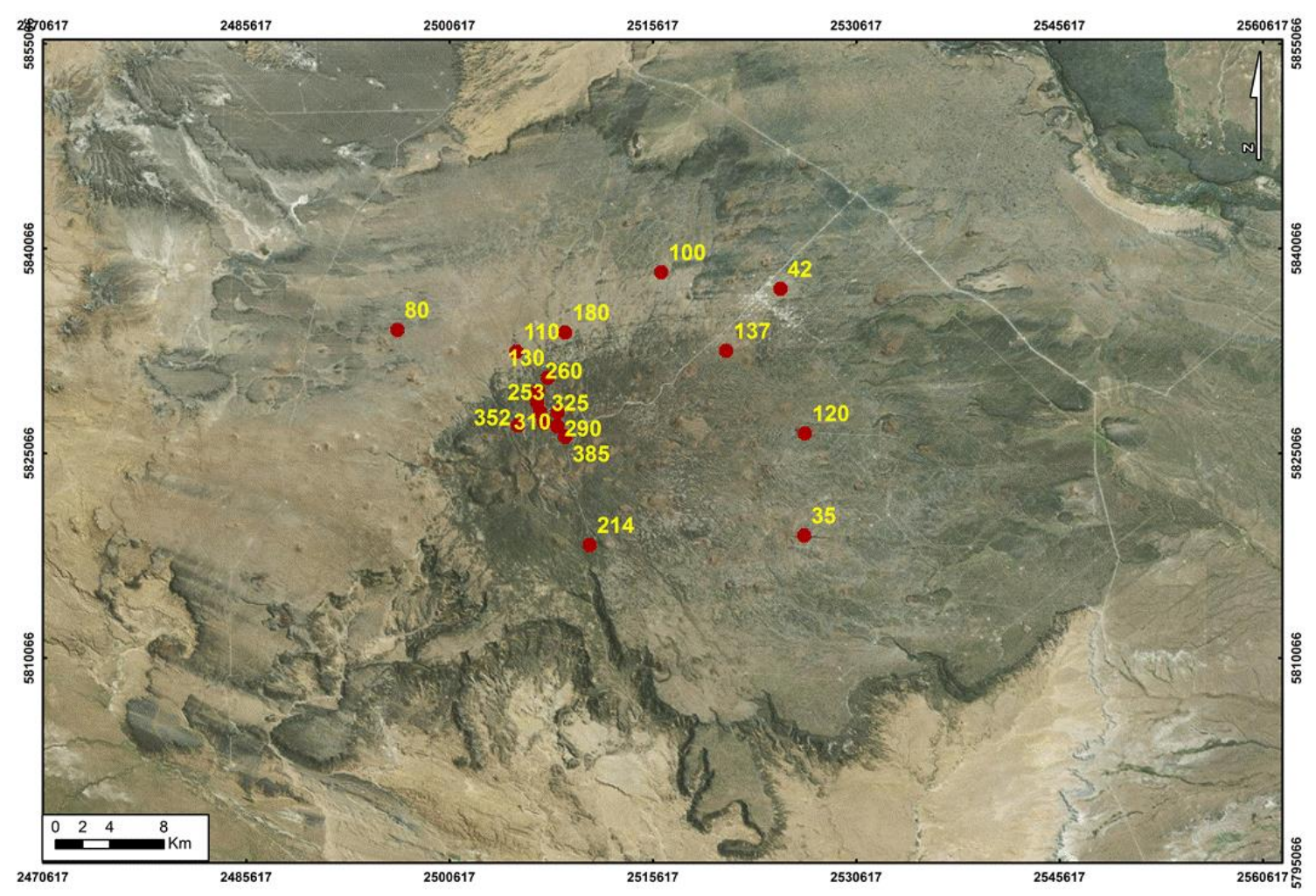

Figura 3.10. Espesores en metros de roca ígnea de la cubierta lávica atravesada por pozos.

\subsubsection{Temperatura del subsuelo y su relación con las propiedades magnéticas}

Como veremos en el capítulo siguiente la temperatura del subsuelo juega un rol importante en las propiedades magnéticas de los minerales y rocas causantes de anomalías magnéticas. En particular la temperatura de Curie, es un parámetro de gran importancia puesto que es la temperatura por encima de la cual un cuerpo ferromagnético pierde su magnetismo, comportándose como un material puramente paramagnético.

Es importante estimar a qué profundidad se encuentra la isoterma de Curie ya que no hay contribución de fuentes magnéticas situadas a profundidades mayores. Para ello, se recopilaron datos de temperatura de fondo de 17 pozos del área del VAM (tabla 3.3). Estos valores fueron tomados de los registros de temperatura que se hacen rutinariamente con los perfiles de pozo. Es conocido que poco tiempo después de la perforación, la pared del pozo se encuentra a una temperatura más baja que la de equilibrio de la roca a esa profundidad, debido a la circulación de lodo que tuvo lugar durante toda la perforación.

Lo que se hace entonces es aplicar una corrección $T_{\text {corr }}$ para obtener una temperatura de fondo "de equilibrio" (ec. 3.16), es decir la temperatura a la cual se aproximaría nuestra medición si se esperara un tiempo suficientemente largo sin circulación (ni producción o inyección) de fluidos en el pozo.

En este caso se utilizó una corrección empírica para la cuenca Neuquina, según la cual 
la temperatura de fondo de equilibrio está dada a partir del valor medido por la expresión (3.17).

$$
\begin{gathered}
T_{e q}=T_{b h}+T_{\text {corr }} \\
T_{\text {corr }}(z)=0.02105 z-13.541
\end{gathered}
$$

donde $T_{b h}$ es el dato "crudo" de temperatura medido (en ${ }^{\circ} \mathrm{C}$ ) y $z$ es la profundidad en metros desde boca de pozo.

La figura 3.11 a muestra las temperaturas "de equilibrio" así obtenidas en función de la profundidad. Se puede ver que concuerdan con la tendencia regional aceptada para la cuenca Neuquina (Sigismondi, 2012), la cual viene dada por la expresión (3.18):

$$
T\left({ }^{\circ} \mathrm{C}\right)=9.5967+0.0443 z
$$

Los valores máximos de temperatura registrados en los pozos de la zona son, a profundidades cercanas a $4 \mathrm{~km}$, del orden de $180{ }^{\circ} \mathrm{C}$, muy por debajo de la temperatura de Curie para los principales minerales magnéticos por lo que se espera que puedan existir

\begin{tabular}{|c|c|c|c|c|c|c|c|c|}
\hline POZO & $x$ & $\mathbf{Y}$ & GL (msnm) & Prof. (mbbp) & Prof. (msnm) & Temp. $\left[{ }^{\circ} \mathrm{F}\right]$ & Temp. $\left[{ }^{\circ} \mathrm{C}\right]$ & Año \\
\hline CAv.x-1 & 2530542.00 & 5832745.89 & 818.3 & 2935.00 & 2116.70 & 175 & 79.45 & 1971 \\
\hline CLM.x-1 & 2534963.00 & 5812946.00 & 752.1 & 3066.50 & 2314.40 & 200 & 93.33 & 1971 \\
\hline CLG.x-1 & 2517472.00 & 5841586.89 & 918 & 2877.00 & 1959.00 & 180 & 82.22 & 1976 \\
\hline LPE.x-1 & 2532908.00 & 5820460.00 & 817 & 2944.60 & 2127.60 & 179 & 81.67 & 1979 \\
\hline \multirow[t]{3}{*}{ LAn.x-1 } & 2510960.03 & 5818339.91 & 1458 & 3513.00 & 2055.00 & 230 & 110.00 & 1981 \\
\hline & & & & 2642.70 & 1184.70 & 162 & 72.20 & 1981 \\
\hline & & & & 2753.00 & 1295.00 & 181 & 82.78 & 1981 \\
\hline CEJ. $x-1$ & 2512426.00 & 5843175.00 & 955 & 3019.00 & 2064.00 & 125 & 51.67 & 1984 \\
\hline VAM.x-1 & 2505664.99 & 5827112.84 & 1676 & 3479.70 & 1803.70 & 230 & 110.00 & 1985 \\
\hline CLB.x-1 & & & & 1376.50 & 359.50 & 130 & 10.33 & 1991 \\
\hline CEI.X-2 & 2532365.00 & 5827292.00 & 836 & 2431.00 & 1595.00 & 170 & 76.67 & 1992 \\
\hline AgL.x-1 & 2516884.00 & 5832553.89 & 1177 & 2899.00 & 1722.00 & 189 & 87.22 & 1994 \\
\hline VAM.x-3 & 2507062.00 & 5828818.00 & 1467 & 3627.30 & 2160.30 & 230 & 110.00 & 1994 \\
\hline CAv.x-3 & 2527969.00 & 5833086.89 & 896 & 1851.00 & 955.00 & 150 & 65.56 & 1996 \\
\hline \multirow[t]{2}{*}{ PDCh.x-1 } & 2521030.98 & 5832577.91 & 1102 & 2172.10 & 1070.10 & falta carrera & & 1996 \\
\hline & & & & 338.80 & -763.20 & 85 & 29.45 & 1996 \\
\hline \multirow[t]{2}{*}{ EV.x-2 } & 2509144.00 & 5833917.93 & 1227 & 3153.00 & 1926.00 & falta carrera & & 2000 \\
\hline & & & & 2565.00 & 1338.00 & & 83 & 2000 \\
\hline
\end{tabular}
fuentes anómalas hasta profundidades mayores.

Tabla 3.3. Datos de temperatura obtenidos de los legajos de pozo. 
En la figura $3.11 \mathrm{~b}$ se grafican las tendencias de temperatura en distintos sectores de la cuenca.

a

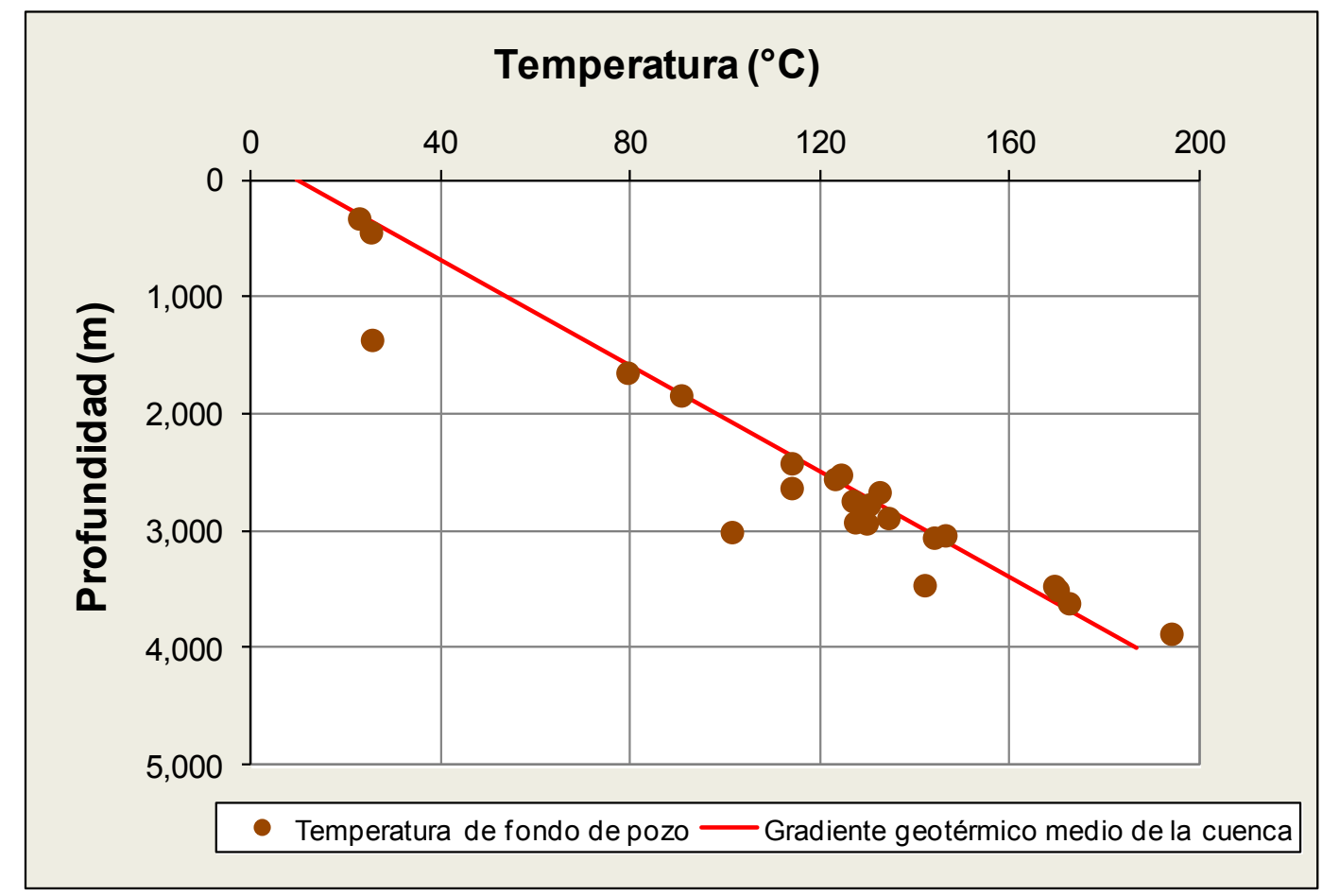

b

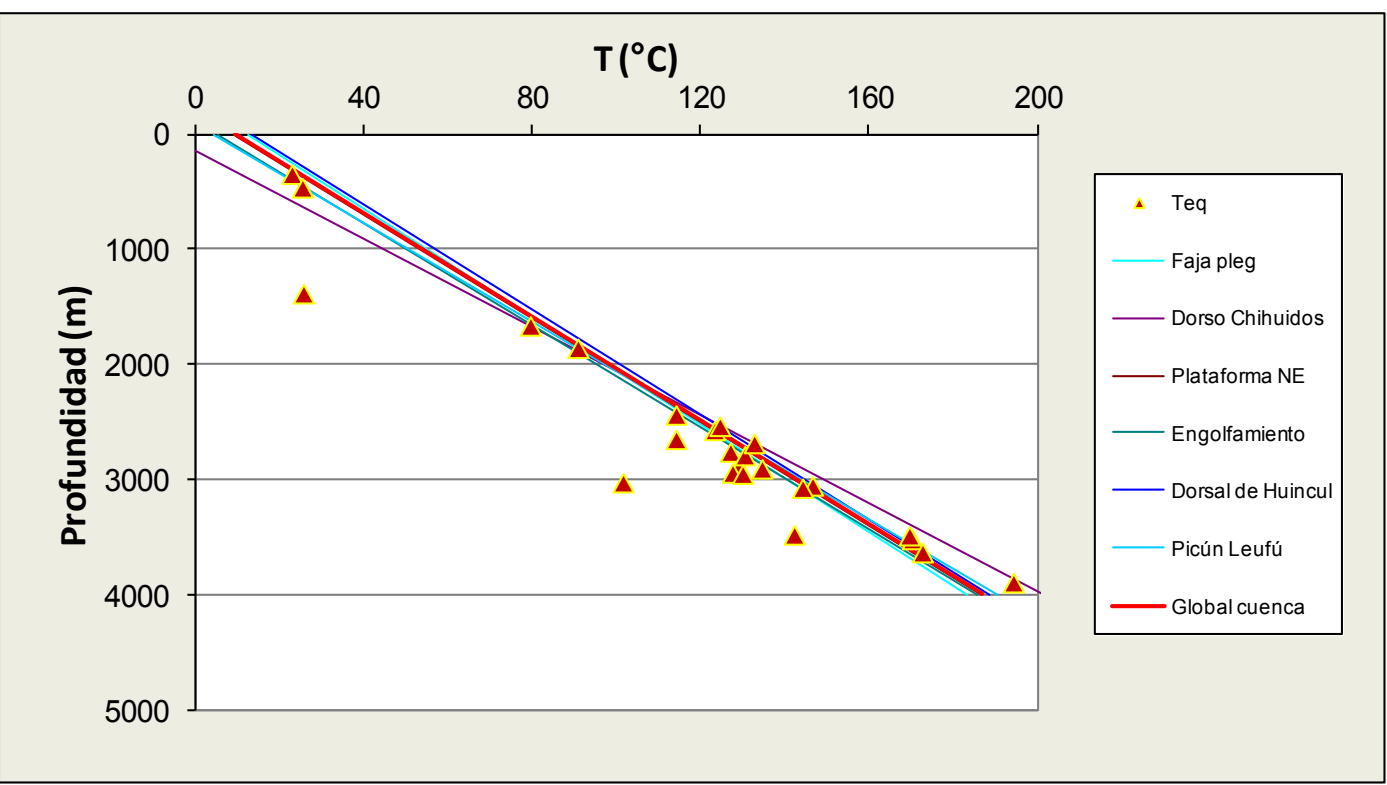

Figura 3.11. Temperatura versus profundidad. a) Los círculos marrones represenan los datos de temperatura de pozo de la zona del Auca Mahuida. En rojo se muestra la recta de tendencia global promedio de la cuenca. b) Los triángulos son los datos de temperatura de los 17 pozos. Las rectas representan a las distintas tendencias geotérmicas zonales dentro de la cuenca (Sigismondi, 2012). 


\section{CAPÍTULO IV}

\section{PROPIEDADES MAGNÉTICAS DE LAS ROCAS}

Las anomalías magnéticas se originan en la inhomogeneidad de la magnetización de las rocas de la corteza terrestre. Esta magnetización se compone de dos contribuciones, inducida y remanente. En las rocas volcánicas, la componente remanente suele ser mayor que la inducida y se debe al comportamiento ferromagnético o ferrimagnético de algunos minerales presentes en las rocas. En este capítulo se hace una breve reseña del comportamiento magnético de los materiales. El aspecto más importante para este estudio es el hecho de que los minerales magnéticos presentan una magnetización remanente (adquirida por diversos mecanismos durante la historia de las transformaciones de la roca y el ambiente) y una magnetización inducida proporcional al campo terrestre actual. La polaridad y dirección de magnetización determinadas en laboratorio de las muestras colectadas en el plateau son consistentes con las inversiones que el campo geomagnético experimentó durante el lapso de tiempo en que el VAM tuvo actividad volcánica. Estas inversiones deben tenerse en cuenta para el análisis del campo de anomalías magnéticas, en particular para la reducción al polo; este análisis se presentará en el capítulo V.

\subsection{Magnetización en las rocas}

La magnetización registrada en las rocas, permite estudiar el campo geomagnético en el pasado. Estudios realizados en magnetismo de rocas proveen las bases teóricas y experimentales para explicar cómo las rocas pueden registrar los campos geomagnéticos pasados. La magnetización de un material $\boldsymbol{M}$ tiene dos contribuciones: una que existe sólo en presencia de un campo externo (magnetización inducida) y aquella que existe en ausencia de campo magnético externo (magnetización remanente).

La presencia de minerales ferromagnéticos o ferrimagnéticos en las rocas hace que sean capaces de adquirir la magnetización remanente o remanencia, así como presentar histéresis en su respuesta magnética al campo ambiente. La magnetización de una roca presenta entonces dos contribuciones, es decir el vector suma de dos componentes (expresión 4.1, figura 4.1):

$$
M=M_{\mathrm{i}}+M_{\mathrm{r}}
$$


donde $\boldsymbol{M}_{\mathrm{i}}$ es la magnetización inducida debido a la presencia de un campo magnético $\boldsymbol{H}$, y $\boldsymbol{M}_{\mathrm{r}}$ la magnetización remanente. A su vez, $\boldsymbol{M}_{\mathrm{i}}=\chi \boldsymbol{H}$, donde $\chi$ es una propiedad del material, llamada susceptibilidad magnética, la cual es adimensional. La susceptibilidad depende de la temperatura en la mayoría de los materiales. La importancia relativa de las dos componentes de la magnetización se suele expresar mediante el coeficiente de Königsberger, que es el cociente entre la intensidad de la magnetización remanente y la inducida, como se indica en la relación (4.2).

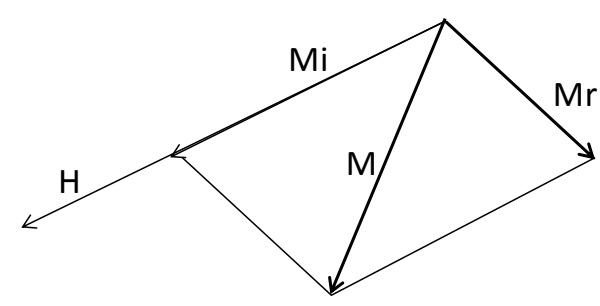

Figura 4.1. Magnetización resultante de la suma de las componentes de magnetización inducida, $M_{\mathrm{i}}, \mathrm{y}$ remanente, $\boldsymbol{M}_{\mathrm{r}}$.

$$
\begin{gathered}
Q=\frac{\left|\boldsymbol{M}_{\mathrm{r}}\right|}{\left|\boldsymbol{M}_{\mathrm{i}}\right|} \\
=\frac{\left|\boldsymbol{M}_{\mathrm{r}}\right|}{|\chi \boldsymbol{H}|}
\end{gathered}
$$

La inducción magnética usualmente es paralela al campo geomagnético local y su adquisición es un proceso reversible sin memoria de los campos magnéticos pasados. Por lo tanto, la componente remanente es la que interesa en paleomagnetismo. La magnetización remanente natural (NRM, por sus siglas en inglés) es la magnetización remanente presente en una muestra de roca previa al tratamiento en laboratorio. La NRM depende del campo geomagnético terrestre y de los procesos geológicos durante la formación e historia de la roca. Generalmente está compuesta por más de una componente. La componente adquirida durante la formación de la roca se llama NRM primaria, y las denominadas componentes secundarias son las adquiridas subsecuentemente a la formación de la roca pudiendo alterar u oscurecer la NRM primara (Butler, 1992). La NRM se puede expresar como la suma de ambas componentes:

$\mathrm{NRM}=\mathrm{NRM}$ primaria $+\mathrm{NRM}$ secundaria 
Las tres formas básicas de NRM primaria son: 1) magnetización termorremanente, adquirida durante el enfriamiento desde altas temperaturas; 2) magnetización química remanente, formada a partir del crecimiento de granos ferromagnéticos debajo de la temperatura de Curie; y 3) magnetización remanente detrítica, adquirida durante la acumulación de rocas sedimentarias que contienen minerales ferromagnéticos detríticos.

La NRM secundaria puede ser el resultado de cambios químicos que afectan los minerales ferromagnéticos, exposición de rayos, o grandes periodos de exposición a campos geomagnéticos subsecuentes a la formación de la roca. La magnetización remanente isotérmica (IRM) es el resultado de la exposición de periodos cortos a fuertes campos magnetizantes a temperatura constante. En laboratorio, la IRM se obtiene a temperatura ambiente generalmente por medio de un campo magnetizante generado por electroimán, mientras que la IRM natural puede ser una componente secundaria de la IRM por exposición a campos magnéticos producidos por rayos.

Otro tipo de magnetización secundaria, es la magnetización remanente viscosa (VRM) la cual se adquiere gradualmente durante la exposición a campos magnéticos débiles, resultado de la acción del campo geomagnético mucho tiempo después de la formación de la roca. Desde el punto de vista paleomagnético, la VRM usualmente se considera ruido indeseable.

\subsection{Minerales magnéticos}

Los minerales magnéticos más importantes son los óxidos de hierro y titanio, que existen en la naturaleza como minerales llamados ferritas (Lowrie, 2007). Su estructura consiste en una red de iones oxígeno en la cual algunos de los sitios intersticiales son ocupados por arreglos regulares de iones ferrosos $\left(\mathrm{Fe}^{2+}\right)$ y férricos $\left(\mathrm{Fe}^{3+}\right)$ e iones de titanio $\left(\mathrm{Ti}^{4+}\right)$. Las proporciones relativas de los tres iones determina las propiedades ferrimagnéticas del mineral (Lowrie, 2007). Estas proporciones pueden representarse como puntos en un diagrama ternario (figura 4.2) cuyos vértices son $\mathrm{FeO}$ (wustita), $\mathrm{TiO}_{2}$ (rutilo) y $\mathrm{Fe}_{2} \mathrm{O}_{3}$ (hematita o maghemita). En este diagrama, las series más importantes de minerales magnéticos quedan representadas por dos líneas: a) la titanomagnetita, que son las soluciones sólidas de fórmula $\mathrm{Fe}_{3-\mathrm{x}} \mathrm{Ti}_{\mathrm{x}} \mathrm{O}_{4}$, y b) la titanohematita, de fórmula $\mathrm{Fe}_{2-\mathrm{x}} \mathrm{Ti}_{\mathrm{x}} \mathrm{O}_{3}$.

Las rocas contienen frecuentemente otros minerales ferrimagnéticos de cierta importancia. La pirrotita es un mineral común de sulfuro de hierro, ferrimagnético en ciertos rangos de composición. La goetita $(\mathrm{FeOOH})$ es un oxi-hidróxido de hierro, un mineral común en sedimentos. Como la hematita, es antiferromagnética pero tiene ferromagnetismo parasitario (momento neto débil que aparece debido a vacancias o defectos cristalinos que hacen que algunos espines estén desapareados). 


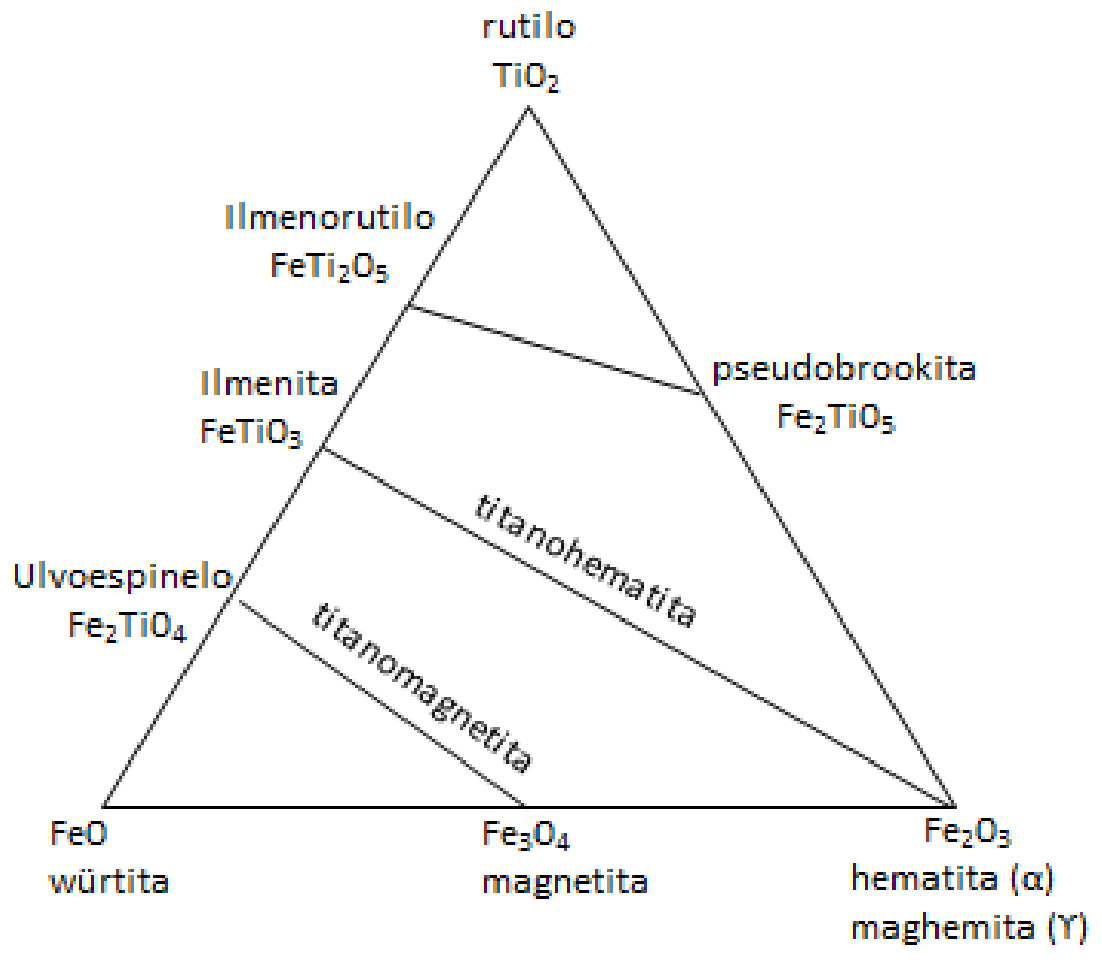

Figura 4.2. Diagrama ternario para los óxidos de hierro, modificado de Lowrie (2007). Las líneas continuas son las series de solución.

\subsection{Muestreo paleomagnético, medición de la NRM y desmagnetización}

En una dada locación sobre la Tierra se pueden observar cambios lentos (de tiempo característico medido en décadas o siglos) en las componentes dipolares y no dipolares del campo geomagnético. Se manifiestan por ejemplo, en variaciones de la inclinación del eje del dipolo, del orden de algunos grados por siglo (Lowrie, 2007). Es necesario promediar en el tiempo esta variación secular para determinar la dirección del campo en una aplicación paleomagnética.

Una primera aproximación razonable es suponer que el campo geomagnético es un dipolo axial geocéntrico (como un imán con su eje alineado con el eje de rotación) cuando estas variaciones se promedian sobre unos $10^{5}$ años.

Es una práctica sugerida (Butler, 1992; Tauxe, 2005) para obtener esta dirección promedio del campo extraer muestras en un número tal que abarquen un intervalo de tiempo del orden de $10^{5}$ años. Por ejemplo, muestreando en unos cien sitios, unas diez muestras por sitio. Se llama sitio a un intervalo dentro de una secuencia o unidad de roca que corresponde a una única unidad de enfriamiento (en roca ígnea) o el mismo horizonte temporal o capa en una roca sedimentaria. Las muestras deben orientarse antes de ser extraídas. El muestreo se hace entonces en niveles jerárquicos: unidad - sitio - muestra orientada. Además, es 
aconsejable muestrear en varias locaciones separadas por varios kilómetros para promediar variaciones locales arealmente.

La extracción de las muestras en el campo se efectúa usando una perforadora de mano (para cortar cilindros de unos $2.5 \mathrm{~cm}$ de diámetro) o cortando bloques irregulares. En todos los casos la muestra debe ser orientada antes de su extracción, donde se determina la inclinación (dip), el acimut (figura 4.3) y coordenadas.

La magnetización remanente de las muestras paleomagnéticas se mide con magnetómetros, los cuales miden las tres componentes del vector magnetización. Los más accesibles son los magnetómetros de tipo spinner, en los cuales la muestra es rotada generando una fuerza electromotriz (FEM) variable en unas bobinas receptoras. Esta FEM es proporcional a la magnetización de la muestra. Otra técnica usual es el magnetómetro criogénico que funciona mediante un dispositivo SQUID (superconducting quantum interference device), de alta precisión pero costoso de adquirir y operar (requiere helio líquido para el sistema superconductor).

Como se mencionó anteriormente, la NRM está formada usualmente por varias componentes adquiridas en tiempos distintos, incluso durante los procedimientos de muestreo y preparación. Entonces, se deben aplicar técnicas de laboratorio que eliminen las componentes no deseadas y aíslen la magnetización primaria, proceso al que se conoce generalmente como "lavado magnético". Las dos técnicas de desmagnetización o lavado magnético más empleadas son la desmagnetización de campo alterno (AF) y la desmagnetización térmica.

Para llevar a cabo la desmagnetización AF se coloca la muestra en un campo magnético alterno cuya amplitud decrece con el tiempo hasta cero, siendo $H_{A F}$ el valor de amplitud máxima. El proceso de barrido con el campo alterno se repite para varios valores (crecientes) de la amplitud máxima $H_{A F}$. Por ejemplo, se inicia con una amplitud de $3 \mathrm{mT}$ y se repite el barrido de campo alterno en pasos hasta un valor de $H_{A F}$ del orden de 70 o $100 \mathrm{mT}$, dependiendo de la muestra. El resultado neto de cada barrido de campo alterno es que todos los momentos magnéticos con valores del campo coercitivo $H_{c}<H_{A F}$ se anulan en promedio. El vector magnetización remanente medido al final de cada paso se grafica de modo de identificar una estabilización. La forma tradicional de hacerlo es graficar para la remanencia resultante en cada paso, por un lado su intensidad para los distintos valores de $H_{A F}$ y por otro lado su dirección en una proyección estereográfica. El método preferido actualmente es el de diagramas vectoriales o de Zijderveld (Butler, 1992).

La desmagnetización térmica consiste en calentar la muestra hasta una temperatura (Tdemag) elevada pero menor que la temperatura de Curie de los minerales ferromagnéticos y luego enfriarla hasta temperatura ambiente en ausencia de campo magnético aplicado (es decir en un recinto blindado). De este modo quedan "desordenados" los granos de menor estabilidad y se anula su contribución a la magnetización. Aquí también se procede por pasos, en los cuales se repiten los ciclos de calentamiento y enfriamiento con temperaturas máximas progresivamente más altas. 


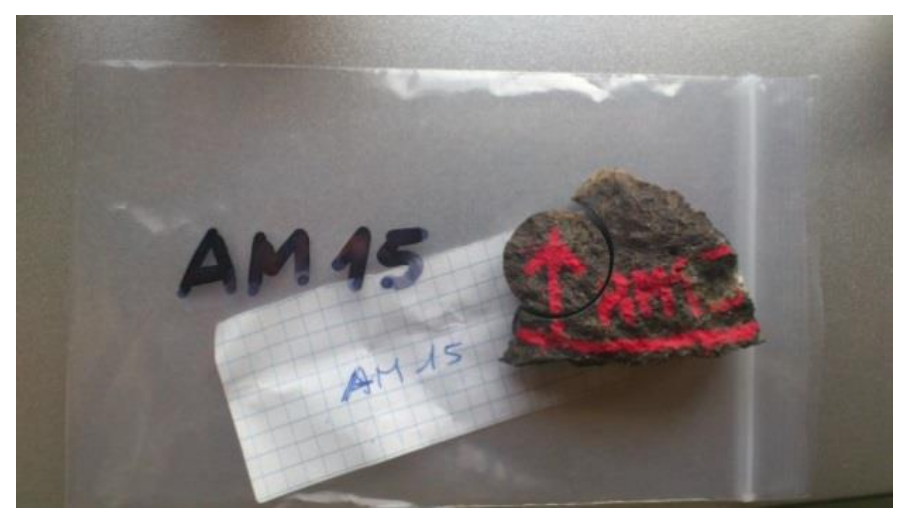

Figura 4.3. Foto de la muestra AM15 con su orientación, tomada durante la campaña.

\subsection{Muestras obtenidas en el complejo volcánico Auca Mahuida}

Con el fin de obtener los valores de magnetización, declinación e inclinación magnética para ser usados en distintos métodos de interpretación, se llevó a cabo una campaña en el plateau volcánico del Auca Mahuida y áreas adyacentes (figura 4.4) en abrilmayo de 2011. Se recolectaron 33 muestras representativas del plateau y para cada una de ellas se midió el buzamiento y la dirección del buzamiento. La susceptibilidad magnética fue medida in situ con un susceptibilímetro de alta sensibilidad (10-7 SI) ZH - SM-30 y a posteriori también en el laboratorio. La composición de las muestras varía de basáltica a traquítica.

Las mediciones de susceptibilidad magnética por unidad de masa y magnetización remanente (tabla 4.1) fueron determinadas en el laboratorio de paleomagnetismo del Istituto Nazionale di Geofisica e Vulcanologia (INGV, Roma, Italia). La susceptibilidad magnética de treinta muestras se midió con un equipo AGICO de Kappabridge (modelo KLY-2), principalmente lavas (86\%) con menores cantidades de escoria de conos y pómez, así como también limolitas y arcilitas de afloramientos del Grupo Neuquén. La magnetización remanente natural (NRM) de las muestras de lava (cortadas en cubos de unos $2 \mathrm{~cm}$ de lado) se midió en un cuarto blindado usando un magnetómetro criogénico DC-SQUID (2G Enterprises, USA). La desmagnetización de las muestras se hizo con campo alterno (AF) hasta $70 \mathrm{mT}$. La mayoría de las muestras mostraron un régimen de estabilidad y por lo tanto permitieron determinar la dirección de su NRM. Los datos de desmagnetización fueron graficados en diagramas ortogonales (Zijderveld, 1967), y las componentes fueron aisladas mediante análisis de componentes principales (Kirschvink, 1980). Los valores de MRN de todas las muestras se encuentran en el rango de 1 a $40 \mathrm{~A} / \mathrm{m}$, lo cual está bien por encima del nivel de ruido del magnetómetro (de unos $10^{-5} \mathrm{~A} / \mathrm{m}$ ). La componente viscosa fue removida en todas las muestras a 10-20 mT, mientras que se aisló una dirección característica de la magnetización remanente en el intervalo de amplitud del campo alterno de 20 a 70 mT. 
También se determinó la declinación, inclinación paleomagnética y el parámetro de Königsberger $(\mathrm{Q})$ que expresa la importancia relativa entre la magnetización remanente $\left(M_{\mathrm{r}}\right)$ y la magnetización inducida $\left(M_{\mathrm{i}}\right)$. La remanencia magnética medida es alta. El 65\% de las muestras tienen polaridad reversa y el coeficiente de Königsberger varía entre 2.1 a 200 (tabla 4.1; Paine et al., 2015). La componente remanente es la predominante para los afloramientos del flujo de lava.

Es muy importante vincular la edad de las rocas del plateau con la escala de tiempo de polaridad del campo geomagnético, para establecer si está de acuerdo con la polaridad obtenida de las muestras de campo.

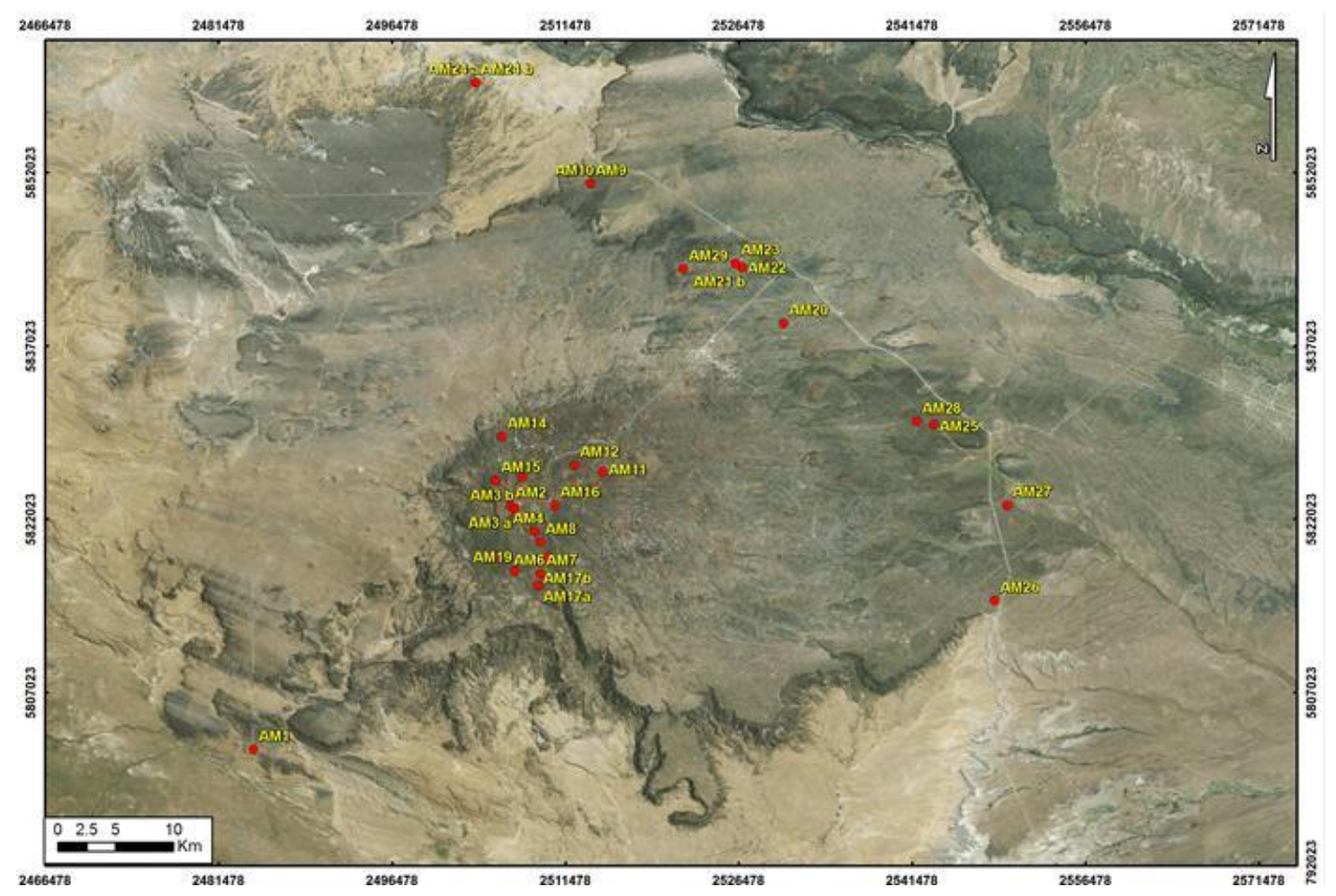

Figura 4.4. Imagen de la distribución de muestras en el campo y medición de la susceptibilidad magnética in situ, puntos rojos sobre plateau. 
Tabla 4.1. Mediciones de las propiedades magnéticas de las rocas en laboratorio e in situ. En color negro se representa la polaridad normal, en blanco, la reversa. NA indica que la polaridad no pudo ser determinada.

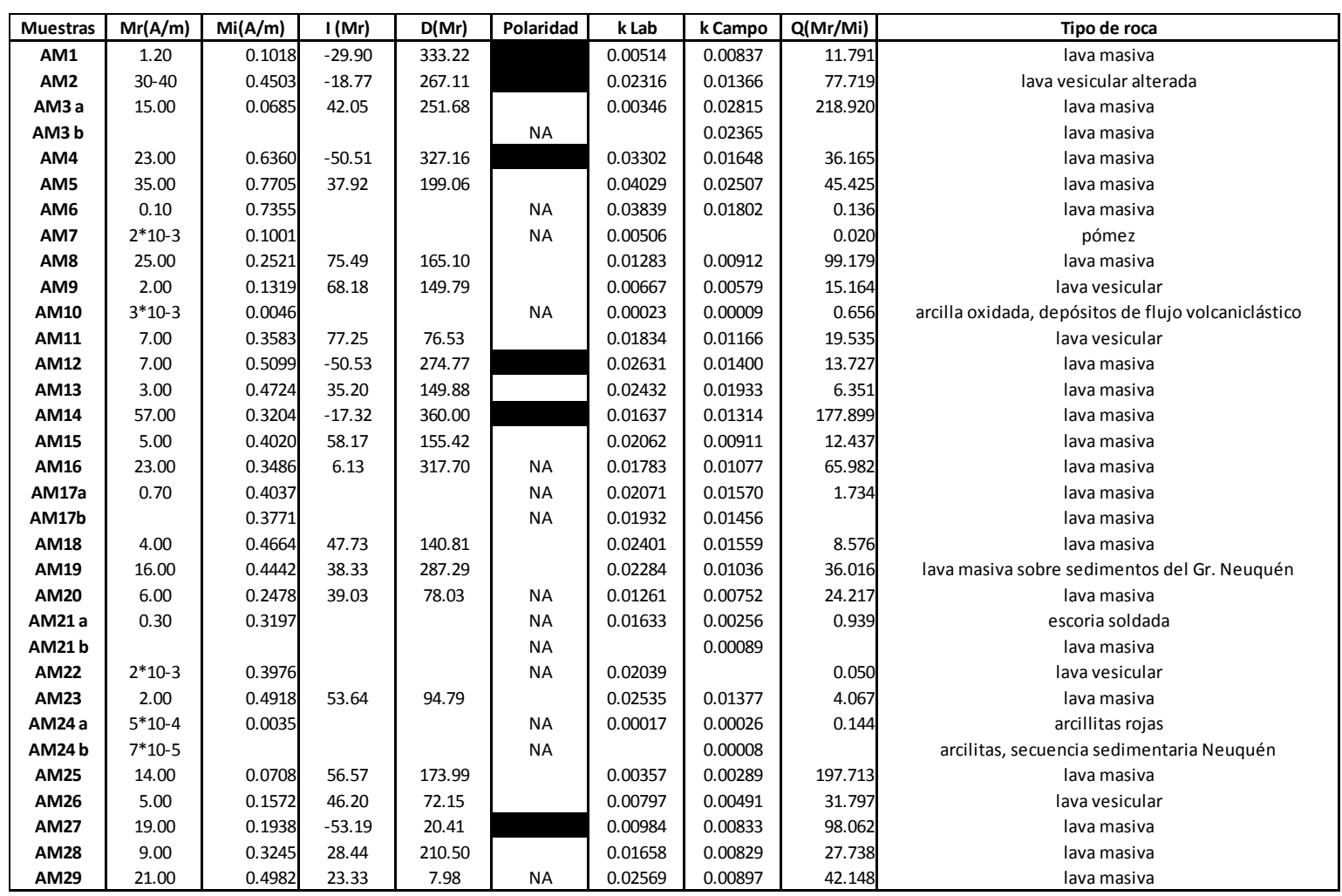

A partir de la década de 1960 fue posible la datación precisa de rocas ígneas del Plioceno-Pleistoceno mediante la técnica de potasio-argón (K-Ar). Se hallaron rocas ígneas de la misma edad de locaciones muy separadas con la misma polaridad. Se compilaron los datos de edad y polaridad de un gran número de rocas ígneas y esto dio lugar al desarrollo de las primeras escalas de tiempo de polaridad geomagnética en el intervalo de 0 a 5 M.a. La incertidumbre propia de los análisis de datación radimétrica por lo general limita la aplicación de la técnica a los 5 M.a. para las determinaciones de polaridad. A esa antigüedad, el error típico de la datación radimétrica se hace comparable a la duración de los intervalos de polaridad constante. Salvo casos excepcionales, se necesitan otras técnicas para descifrar la escala temporal de la polaridad del campo para tiempos mayores a 5 M.a. (Butler, 1992). Por ejemplo Cox (1982) desarrolló una escala para el Cretácico Tardío - Cenozoico entre 0 y 118 M.a. (figura 4.8). Al menos para el Cenozoico se puede afirmar que las edades absolutas de los crones de polaridad magnética se conocen con una precisión de \pm 2 M.a. Es importante notar que la edad relativa de los crones se conoce con mucha mayor precisión, del orden de los $10^{4}$ años.

De acuerdo con dataciones radimétricas realizadas en rocas de la colada volcánica mencionadas en el Capítulo II (Kay et al., 2006; Ramos and Folguera, 2010), las muestras recolectadas, pertenecientes a las unidades de lavas descriptas en el mapa geológico 
(Delpino, 2002) se corresponderían principalmente con los eventos de polaridad reversa perteneciente al cron Matuyama. En la figura 4.5 se grafica este período en la escala temporal de polaridad geomagnética del Plioceno-Pleistoceno de Mankinen \& Dalrymple (1979), Cox (1982) entre otros.

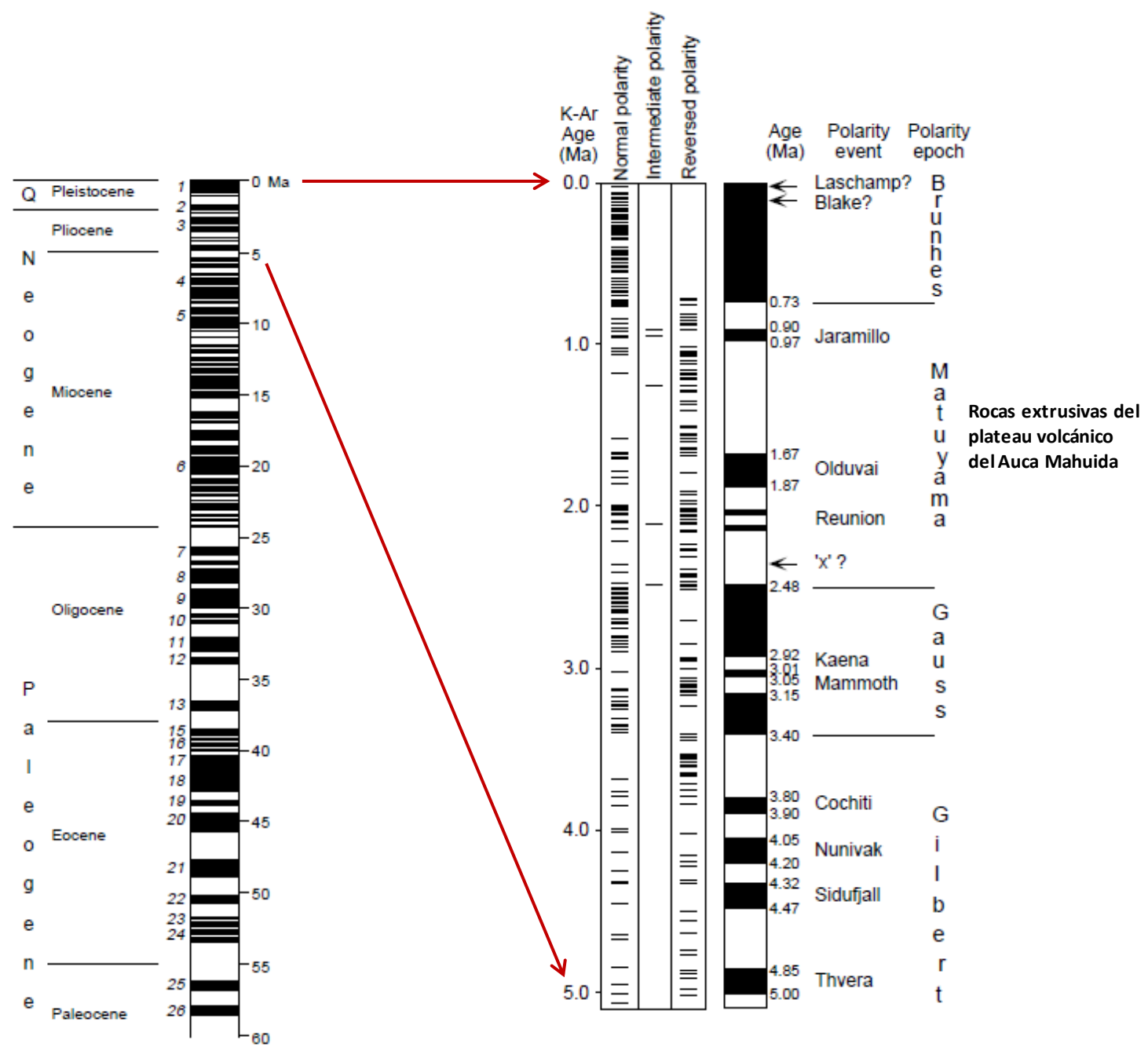

Figura 4.5. Modificado de Butler, 1992. Izquierda: Escala de Cox (1982) de los primeros 60 M.a. Derecha: Escala de Mankinen \& Dalrymple (1979) basada en determinaciones de edad por KAr, polaridad paleomagnética e información complementaria de perfiles marinos de anomalías magnéticas y de datos paleomagnéticos de coronas de lecho marino profundo. Cada línea horizontal en las columnas indicadas como polaridad normal, polaridad intermedia o polaridad reversa representa una roca ígnea Las flechas indican intervalos cortos de polaridad llamados "excursiones" geomagnéticas; los números a la derecha de la columna de polaridad indican las edades interpretadas de los límites entre polaridades distintas. 


\subsection{Muestras de corona del pozo VAM-28}

Se han realizado mediciones de susceptibilidad y remanencia magnética en cinco muestras extraídas en la corona del pozo VAM-28d (figura 4.6). Todas las mediciones se realizaron en el Laboratorio de Paleomagnetismo Daniel Valencio de la Universidad de Buenos Aires, en el mes de abril de 2015. Se analizaron dos rocas ígneas de composición basáltica (figura 4.7) y tres rocas sedimentarias. Las muestras de diques basálticos pertenecen a la Formación Mulichinco, a una profundidad de $2820 \mathrm{mbnm}$ (metros bajo nivel medio del mar) aproximadamente. Se cortaron todas las muestras en cilindros de $25.4 \mathrm{~mm}$ de diámetro y alturas distintas de acuerdo a lo que permitió la forma y tamaño de la muestra original (figura 4.8).

La magnetización remanente natural MRN se midió con un equipo JR-6 AGICO Dual Speed Spinner magnetometer (figura 4.9). Se usaron cuatro posiciones para la medición. Este proceso elimina cualquier valor no compensado del portamuestras y reduce el error de las mediciones causadas por una inadecuada forma del espécimen y el ruido del instrumento. Una muestra normal para este instrumento consiste de un cubo de $20 \mathrm{~mm}$ o de un cilindro de $25.4 \mathrm{~mm}$ de diámetro y $22 \mathrm{~mm}$ de largo. Como en este trabajo se usaron cilindros de diferentes alturas se corrigió el resultado de la medición al volumen estándar.

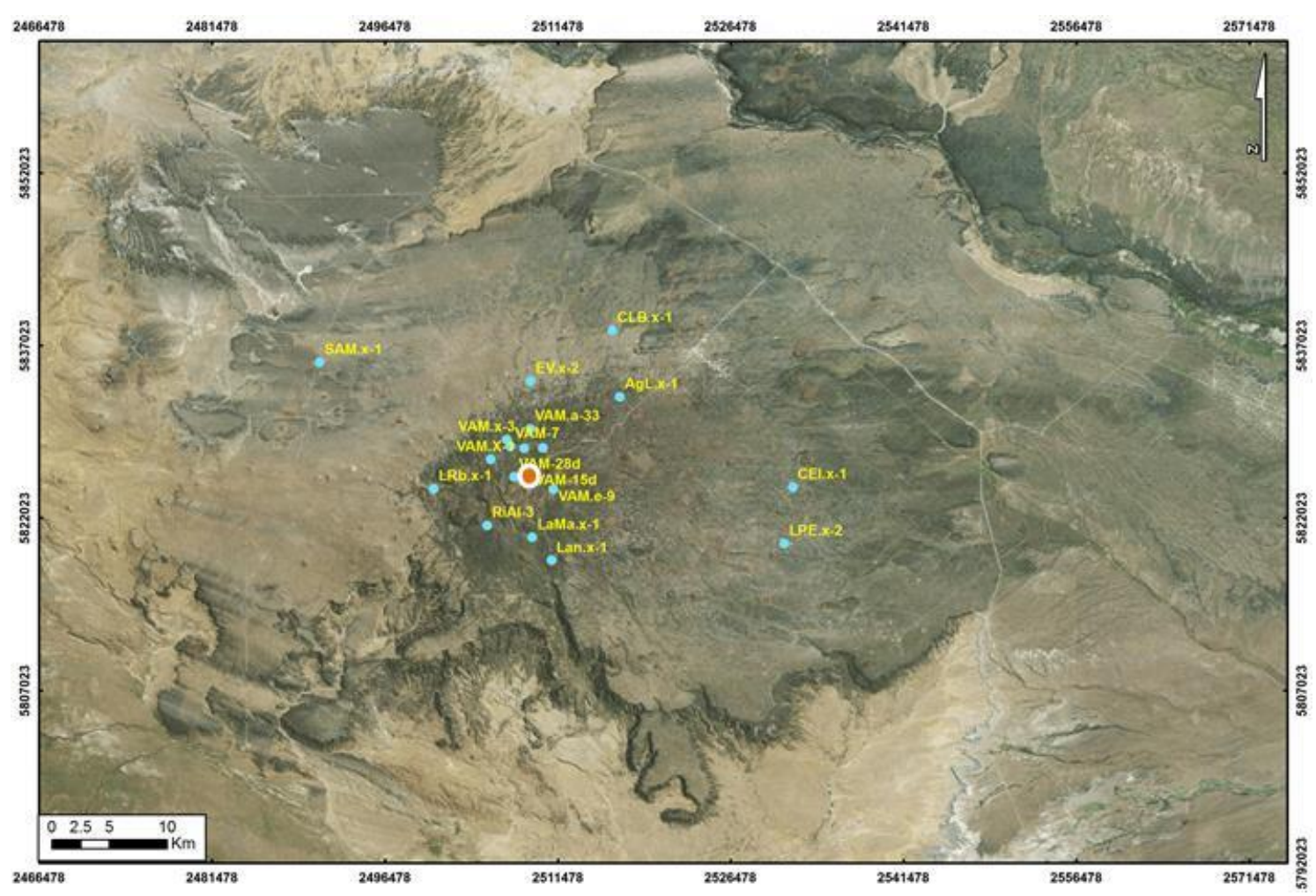

Figura 4.6. Ubicación del pozo VAM-28 (círculo naranja en la imagen satelital). 


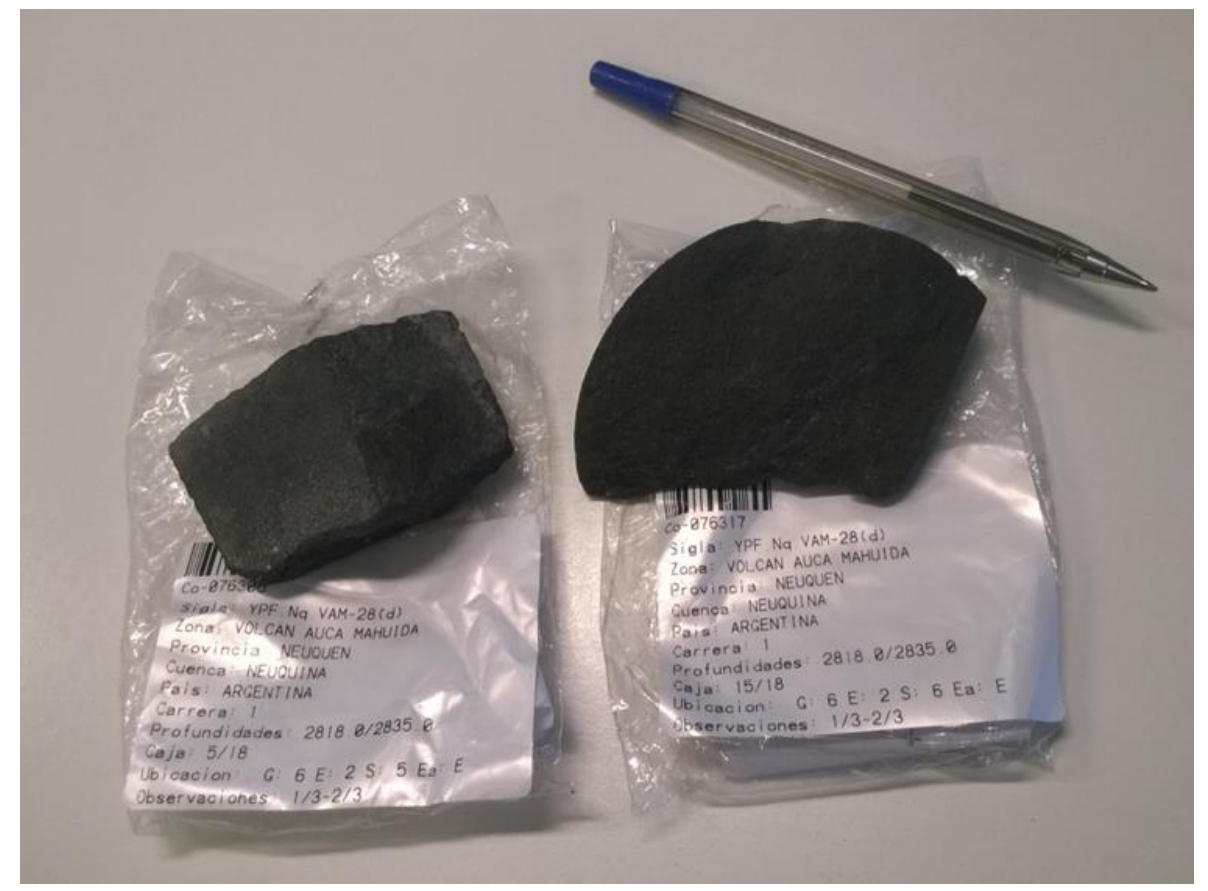

Figura 4.7. Fragmentos de diques basálticos atravesados por el pozo.

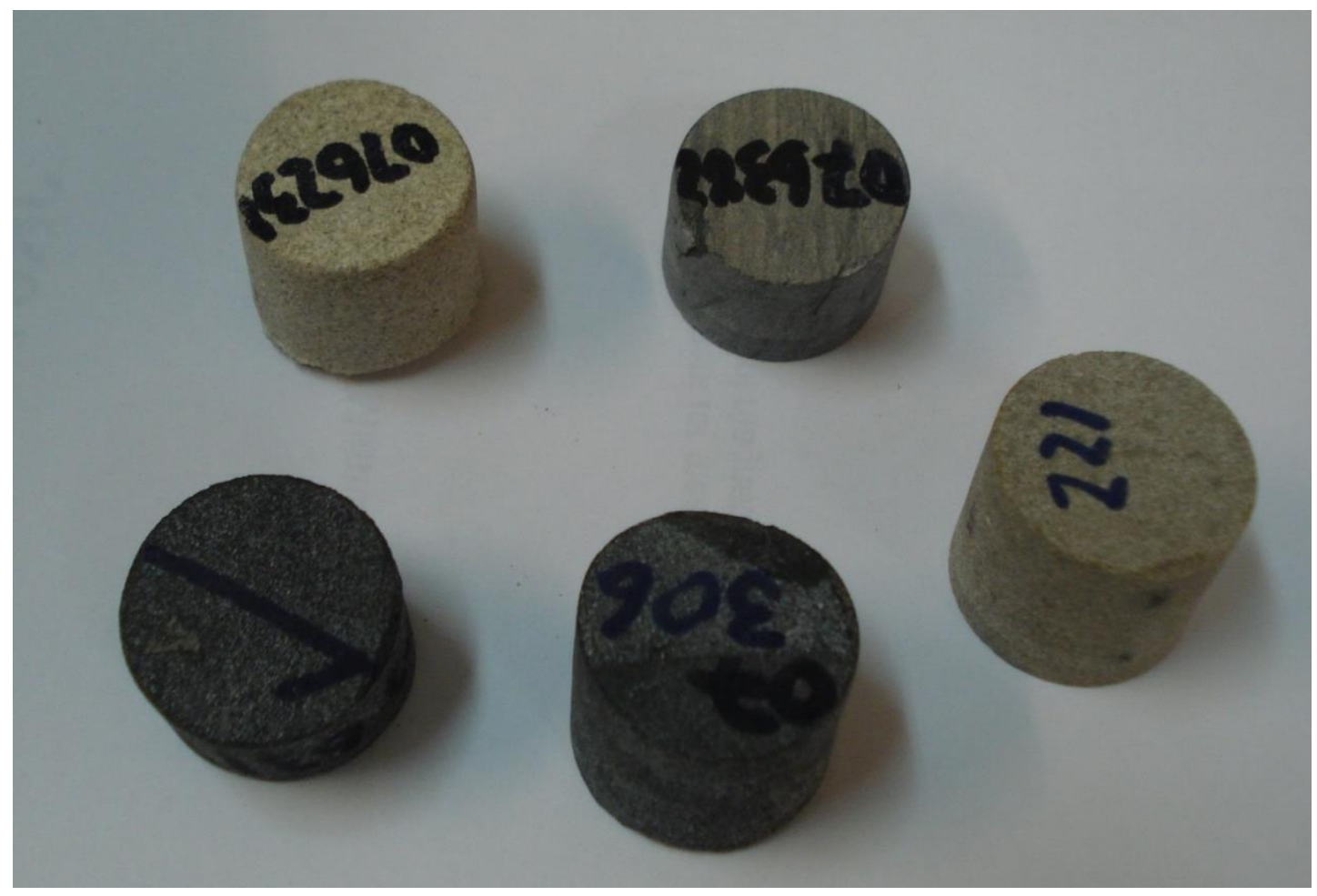

Figura 4.8: Muestras cortadas listas para medir. 

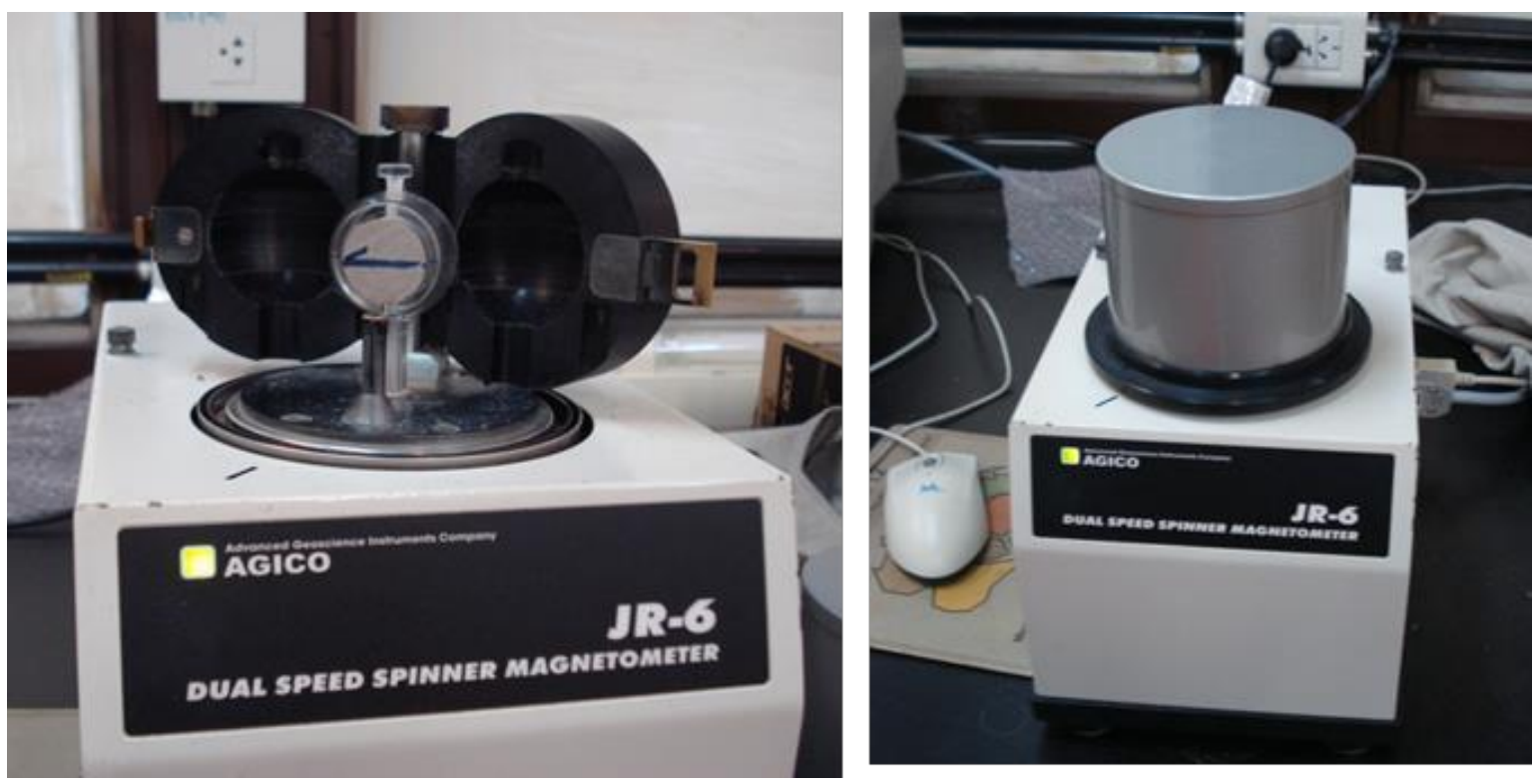

Figura 4.9. Magnetómetro JR-6 AGICO Dual Speed Spinner magnetometer.

Los valores de suscpetibilidad magnética y NRM para los diques basálticos son muy bajos (tabla 4.2). Se hicieron lavados magnéticos por campos alternos AF a 5 y $10 \mathrm{mT}$ en ambas muestras con un desmagnetizador por campos alternos LDA (figura 4.10). Mostraron una leve tendencia hacia el origen pero los valores de NRM luego de cada lavado se mantuvieron igualmente muy bajos, del mismo orden de magnitud del inicio de la medición.

La susceptibilidad magnética por unidad de volumen de cada muestra se midió con un equipo Multifunction MFK1-FA KAPP Bridge.

Tabla 4.2. Valores de las mediciones de NRM y susceptibilidad magnética.

\begin{tabular}{|c|c|c|c|c|c|}
\hline Muestra & $\operatorname{MRN}(\mathrm{A} / \mathrm{m})$ & Precisión & Susceptibilidad & $\operatorname{Err}(\%)$ & Litología \\
\hline $07-306$ & $554.2 \mathrm{E}-06$ & $0.6^{\circ} \quad 3.6 \%$ & 0.00006 & 0.10210 & dique basáltico \\
\hline 07-6317 & 395.0 E-06 & $2.0^{\circ} \quad 1.2 \%$ & 0.00003 & 0.24020 & dique basáltico \\
\hline 07-6382 & 521.0 E-06 & $3.1^{\circ} \quad 3.7 \%$ & 0.00009 & 0.07970 & arcilita \\
\hline $07-6231$ & 1.130 E-03 & $1.7^{\circ} \quad 1.2 \%$ & 0.00002 & 0.40480 & arenisca \\
\hline $07-6221$ & 1.929 E-03 & $3.5^{\circ} \quad 2.6 \%$ & 0.00004 & 0.14810 & arenisca \\
\hline
\end{tabular}


Los valores de suscpetibilidad magnética y MRN de estos seis especímenes seleccionados de la corona, son mucho más bajos que los de las muestras de superficie. Esto puede deberse a que la roca en esta posición pudo ser alterada por factores extrínsecos, como algunos procesos físico-químicos que afectan sus propiedades magnéticas. No obstante, debe tenerse en cuenta que muestras de un solo pozo no necesariamente son representativas del volumen total de roca ígnea.

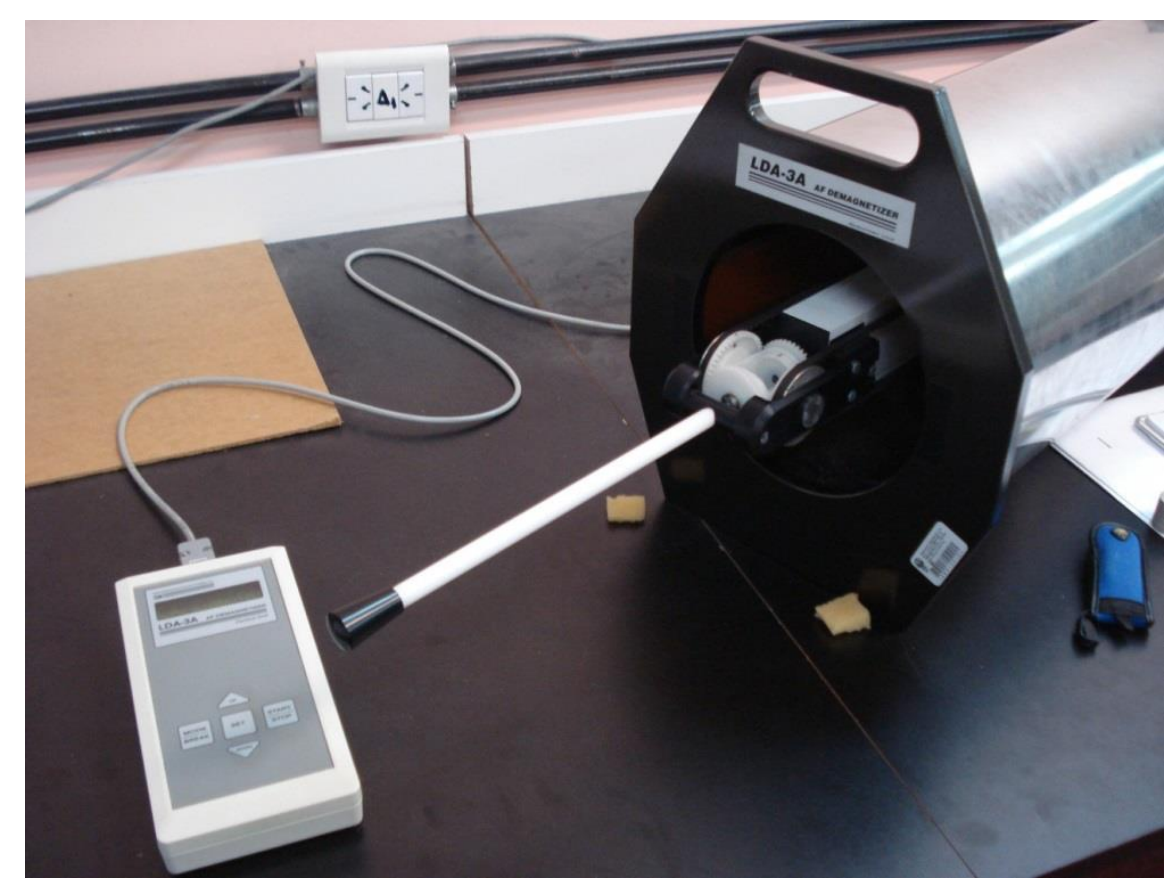

Figura 4.10. Desmagnetizador por campos alternos, AF Demagnetizer LDA. 


\section{CAPÍTULO $V$}

\section{ANÁLISIS E INTERPRETACIÓN DE LAS ANOMALÍAS MAGNÉTICAS}

Los relevamientos magnéticos constituyen una eficaz herramienta de investigación en ambientes volcánicos donde los métodos sísmicos están ausentes o son de limitada utilidad debido a la alta impedancia acústica de la cubierta volcánica superior o a la topografía. Los resultados obtenidos deben combinarse con otros métodos geofísicos e información directa del subsuelo (pozos) ya que la interpretación por sí sola no es unívoca debido a la ambigüedad de los campos potenciales (hay infinitas configuraciones plausibles de fuentes que producen la misma respuesta). En este capítulo se presentan los resultados de la aplicación de distintas técnicas de análisis de los campos de anomalías magnéticas.

\subsection{Campo de anomalías magnéticas}

En los relevamientos magnetométricos en general se mide la magnitud del campo magnético total sin información sobre la dirección. La anomalía de campo total se calcula a partir de las mediciones de campo total corregido de efectos externos restando la magnitud del campo principal, usualmente obtenido del modelo del Campo de referencia geomagnético internacional (IGRF- Mandea \& Macmillan, 2000), adecuado para la fecha del relevamiento. La anomalía escalar de campo total está dada entonces por ecuación (5.1):

$$
\Delta T=\left|\boldsymbol{B}_{\boldsymbol{c}}\right|-|\boldsymbol{F}|
$$

donde $\boldsymbol{B}_{\boldsymbol{c}}$ representa el vector de campo total en un punto cualquiera corregido por efectos externos y $\boldsymbol{F}$ es el vector campo principal en el mismo punto.

El rango típico de intensidad de las anomalías corticales medidas en relevamientos aéreos, es de pocos nT y puede alcanzar el orden de los $10000 \mathrm{nT}$, mientras que las longitudes de onda están en el rango de unos pocos metros a decenas de kilómetros.

Las anomalías provenientes del relevamiento de 2001 tienen un rango de amplitudes comprendido entre -120 hasta 70 nT. Están concentradas alrededor del cráter central, tienen 
carácter dipolar y en general tienen sus lóbulos positivos y negativos ubicados al sur y norte, respectivamente, en dirección opuesta a la esperada para anomalías en el hemisferio sur (figura 5.1). Siguiendo el uso más frecuente de los términos en la literatura, en esta tesis se denominará TMI al campo total de anomalías magnéticas.

La cobertura lávica en el campo del volcán Auca Mahuida (VAM), dificulta la separación de las señales provenientes de distintas fuentes. Por ello es necesario resolver la ambigüedad presentada por el hecho de que las anomalías podrían ser generadas por fuentes ubicadas en la cubierta volcánica superior y también por fuentes emplazadas debajo de la cubierta volcánica, tales como intrusivos. Este segundo tipo de fuentes son significativas desde el punto de vista de la interpretación estructural del volcán. En los apartados subsiguientes se estudian y aplican las técnicas más relevantes para llevar a cabo la interpretación del mapa de anomalías magnéticas.

Para obtener los datos magnéticos o gravimétricos digitales en forma de grilla, es necesario hacer interpolación y algunas veces extrapolación (para cubrir los huecos en el relevamiento) a partir del conjunto de datos observados. Éstos pueden estar distribuidos en un arreglo uniforme, como es el caso de relevamientos aéreos o tener una disposición aleatoria como en una adquisición terrestre (Naidu and Mathew, 1998).

El grillado de los datos de anomalías magnéticas se realizó con el algoritmo de mínima curvatura. Este método de interpolación tiene la ventaja de eliminar las irregularidades de longitud de onda corta, dando un resultado suave que preserva los rasgos principales del campo. En general, este procedimiento de grillado produce resultados aceptables siempre que los datos estén uniformemente distribuidos, que es el caso de estudio. El tamaño de celda se tomó de acuerdo con el intervalo de muestreo nominal $S$, dado por la ecuación (5.2):

$$
S=\sqrt{\frac{\left(X_{\max }-X_{\min }\right)\left(Y_{\max }-Y_{\min }\right)}{N}}
$$

donde el numerador es el área de la grilla y el denominador $N$ el número de puntos de la misma. De acuerdo a un criterio empírico, se recomienda usar $1 / 2$ o $1 / 4$ del muestreo nominal (Geosoft, 2008). Teniendo en cuenta un área de $1400 \mathrm{~km}^{2}$ y 3436 puntos magnetométricos, se tomó un tamaño de celda de $250 \mathrm{~m}$. 


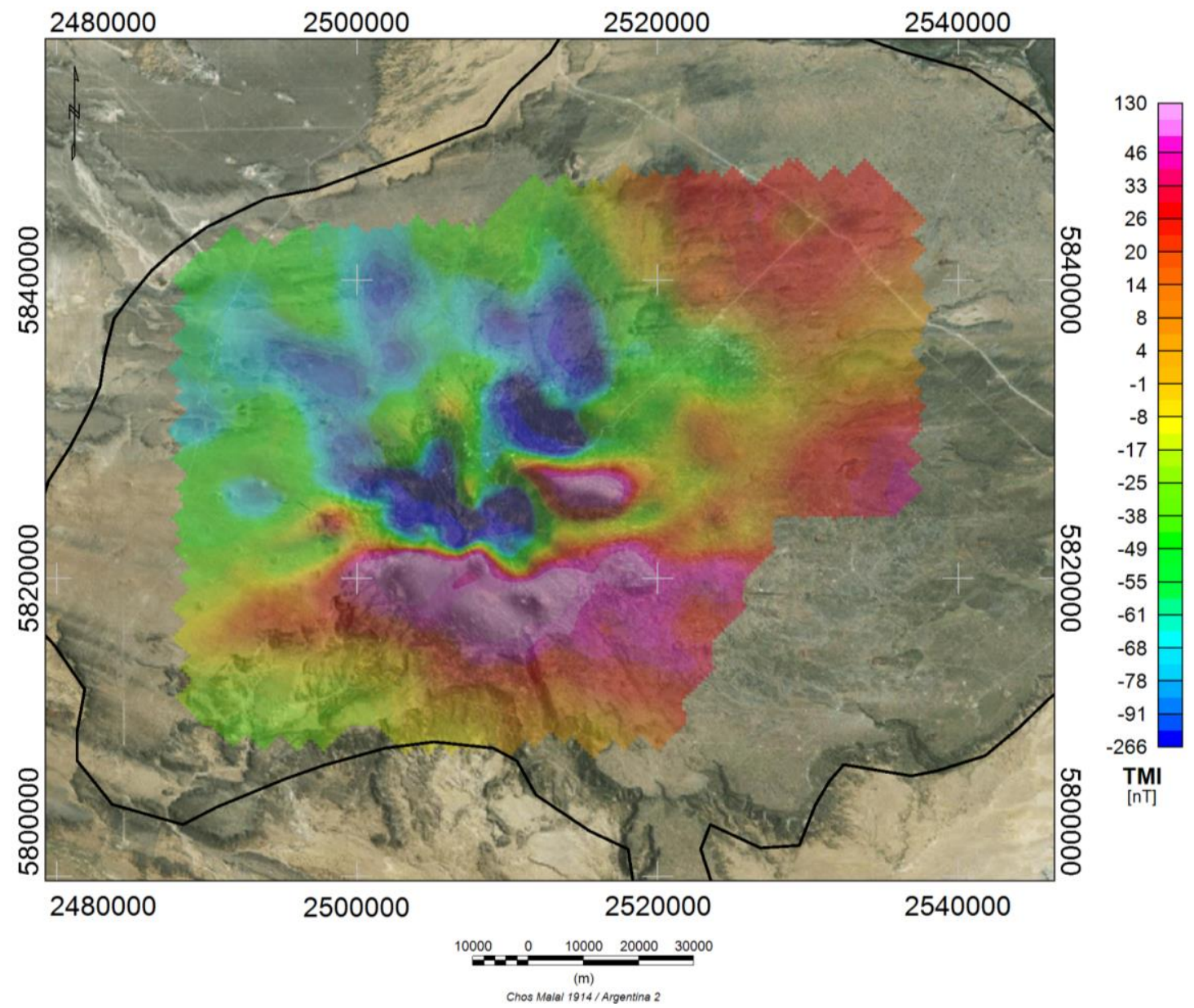

Figura 5.1. Mapa de anomalías magnéticas TMI correspondiente al relevamiento aeromagnético 2001, superpuesto a imagen satelital.

\subsection{Análisis espectral del campo de anomalías magnéticas}

El análisis de Fourier es un procedimiento clásico en métodos potenciales para obtener información de la profundidad de las fuentes de anomalías y para la implementación de técnicas de procesamiento. Los campos potenciales son funciones continuas de las coordenadas espaciales, las que son muestreadas en las etapas de adquisición y procesamiento de los datos. Este hecho impone ciertas limitaciones que hacen imposible obtener el espectro completo de longitudes de onda. El espaciamiento de los datos y la extensión geográfica del relevamiento impone restricciones sobre los números y longitudes de onda detectables en el análisis. De este modo existe una longitud de onda mínima (longitud de onda de Nyquist) definida por el intervalo de muestreo, y una longitud de onda máxima que está condicionada por las dimensiones del área de trabajo (expresiones (5.3) y (5.4)). Conociendo estas limitaciones, se debe tener en cuenta cuales son las longitudes de onda 
asociadas a las fuentes que podemos detectar. Las longitudes de onda de las anomalías magnéticas están relacionadas con el tamaño, forma y profundidad de dichas fuentes.

En general se trabaja con información que se dispone en forma de arreglo bidimensional o grilla, con lo cual para posicionar los datos son necesarias dos coordenadas independientes. Podemos entonces escribir para el caso bidimensional:

$$
\begin{aligned}
& 2 \Delta x \leq \lambda^{x} \leq X \\
& 2 \Delta y \leq \lambda^{y} \leq Y
\end{aligned}
$$

donde $\Delta x$ y $\Delta y$ representan los intervalos de muestreo; y $X$ e $Y$ son las longitudes en las direcciones de $x$ e $y$, respectivamente.

Los números de onda están dados por las siguientes expresiones:

$$
\begin{gathered}
k_{\text {max }}^{x}=\frac{1}{2 \Delta x}, k_{\text {max }}^{y}=\frac{1}{2 \Delta y} \\
k_{\text {min }}^{x}=\frac{1}{X}, k_{\text {min }}^{y}=\frac{1}{X}
\end{gathered}
$$

El criterio de Nyquist establece que para poder recuperar una señal de banda limitada, la frecuencia de muestreo tiene que ser al menos el doble de la máxima frecuencia. Esto es, si $\lambda_{\min }^{x}$ y $\lambda_{\min }^{y}$ son la mínima longitud de onda presente en la función continua correspondiente a la máxima frecuencia o número de onda $k^{x}{ }_{\text {max }}, k^{y}{ }_{\text {max }}$, los intervalos de muestro deberán satisfacer (5.5):

$$
\Delta x_{\max } \leq \frac{\lambda_{\min }^{x}}{2}, \Delta y_{\max } \leq \frac{\lambda_{\min }^{y}}{2}
$$

Es decir, las mínimas longitudes de onda visibles estarán limitadas por el espaciamiento utilizado.

Cuando no se respeta el criterio anterior se estaría submuestreando la información y se puede producir el fenómeno de aliasing. Esto se debe a que las longitudes de onda más cortas no estarán bien representadas y al intentar recuperar la señal original aparecerán como una componente de larga longitud (baja frecuencia).

El intervalo de muestreo de los datos aeromagnéticos de alta resolución del VAM 0.5 $\mathrm{km}$ x $0.5 \mathrm{~km}$ ) y las dimensiones del área (35 km x $40 \mathrm{~km}$, aproximadamente), determinan el mínimo y máximo números de onda $\left(k_{\min }, k_{\max }\right)$ de las anomalías registradas y también la profundidad máxima de investigación. Por lo tanto, las anomalías contienen valores de $k$ 
comprendidos entre $2.810^{-2} \mathrm{~km}^{-1}$ y $1 \mathrm{~km}^{-1}$. Las máximas longitudes de onda son 35 y $40 \mathrm{~km}$, mientras que el número de onda de Nyquist corresponde a una longitud de onda mínima de 1 $\mathrm{km}$.

En los campos potenciales geofísicos, los cuales en general no tienen limitación de banda, el ancho de banda efectivo (el que contiene por ejemplo el 99\% de la potencia) depende de la profundidad de la fuente. Por lo tanto la distancia requerida entre puntos de un muestreo gravimétrico o magnético dependerá de la profundidad esperada de las fuentes. Existe, bajo ciertas suposiciones, una relación simple entre la profundidad $h$ y el radio $s_{o}$ que contiene el 99\% de la potencia espectral de una fuente: $h . s_{o}=3.345$ (Naidu and Mathew, 1998).

\subsection{Técnicas de análisis de las anomalías magnéticas}

En general, los mapas de las anomalías magnéticas son la expresión de un conjunto de fuentes superpuestas cuya contribución individual es difícil de aislar. Las anomalías residuales de interés son habitualmente distorsionadas por los efectos regionales de fuentes más profundas y extensas, así como por el ruido de longitudes de onda corta producido por fuentes más someras. Numerosos métodos encontrados en la literatura, han sido tratados en la separación y resalto de ciertos rasgos de las anomalías. La separación de las anomalías residuales, es un proceso subjetivo, el cual depende mayormente de la experiencia del intérprete y constituye una de las mayores limitaciones de la aplicación de los métodos potenciales. Estas técnicas eliminan del campo de anomalías, todas aquellas que no cumplen alguna condición requerida para la anomalía de interés.

El proceso de separación se basa en considerar características del campo de anomalías tales como amplitudes, dimensiones horizontales, gradientes, atributos direccionales y correlaciones con variaciones geológicas o geofísicas. Las técnicas de realce $o$ resalto de anomalías, en cambio acentúan ciertas características de las anomalías para facilitar su interpretación. El resalto distorsiona las anomalías, por lo que su uso se limita en general, a una inspección visual cualitativa de análisis e interpretación (Hinze et al., 2013). Ejemplos de resalto de anomalías son la corrección topográfica, aplicación de filtros, continuación analítica, reducción al polo y señal analítica entre otros.

La elección del procedimiento más adecuado para la localización de la anomalía magnética residual dependerá de la naturaleza del campo de anomalía, del objetivo del relevamiento y la calidad y cobertura de los datos. A lo largo de este capítulo se aplicarán varios de los métodos analíticos existentes, elegidos en base a las características del campo de anomalías proveniente de los datos aéreos de alta resolución del volcán en estudio. 


\subsubsection{Efecto topográfico de una placa uniformemente magnetizada}

Existen varios factores que producen variaciones espaciales locales en el campo magnético, que pueden distorsionar las anomalías magnéticas de interés en un relevamiento. En áreas volcánicas las fuentes someras o de afloramiento (por ejemplo domos y conos) e importantes pendientes topográficas, sumado a la presencia de elevadas magnetizaciones, pueden producir un efecto magnético capaz de encubrir y modificar a las anomalías de origen más profundo. En los casos en que se puede reconocer fácilmente una correlación directa entre rasgos topográficos y magnéticos se dice que existe un efecto magnético topográfico, el cual debería ser removido de los mapas de anomalías magnéticas. Una de las técnicas utilizadas, consiste en la sustracción del efecto magnético sintético resultado de la topografía a los datos originales (Blanco, 1997). La contribución de esas fuentes superficiales se puede calcular tomando en cuenta directamente la configuración del terreno y la magnetización de las rocas de la superficie. El algoritmo propuesto por Parker (1972), el cual supone un espesor uniforme y topografía irregular, ha sido adaptado por Blakely (1981) para el caso de una fuente con espesor no uniforme (Apéndice B). Se parte de un modelo digital de elevación del terreno (DEM) y se considera una placa paralela a la topografía con dirección de magnetización constante e intensidad variable sólo en la dirección horizontal.

Para el caso del VAM se hizo la estimación del efecto topográfico con el fin de identificar las posibles fuentes relacionadas con la topografía. Se tuvo en cuenta ambas magnetizaciones, inducción y remanencia, para una placa cortical cuya superficie superior coincide con el Modelo Digital de Elevación de alta resolución provisto por YPF S. A., y la inferior es una superficie situada $500 \mathrm{~m}$ por debajo de la elevación topográfica y paralela a la misma. El área elegida es una grilla cuadrada de $125 \mathrm{~km}$ x $125 \mathrm{~km}$, cuyo centro corresponde al edificio central del VAM, cubriendo todo el plateau volcánico del VAM y una amplia área circundante. Los parámetros utilizados son los siguientes:

\section{Inducción}

$\mathrm{I}=-35^{\circ}$

$\mathrm{D}=10^{\circ}$

$\mathrm{k}=0.11 \mathrm{SI}$ (correspondiente a una magnetización de $M_{\mathrm{i}}=2.19 \mathrm{~A} / \mathrm{m}$ para un campo $B=25031$ nT)

$$
\begin{aligned}
& \text { Remanencia } \\
& \mathrm{I}=35^{\circ} \\
& \mathrm{D}=190^{\circ} \\
& M_{\mathrm{r}}=2 \mathrm{~A} / \mathrm{m}
\end{aligned}
$$

El coeficiente de Königsberger es de Q=0.91

El valor de magnetización de $2 \mathrm{~A} / \mathrm{m}$ fue tomado en base a los valores obtenidos de las 
muestras de campo y el espesor de acuerdo a los máximos espesores de los basaltos de superficie.

El valor de magnetización de $2 \mathrm{~A} / \mathrm{m}$ produce un rango de intensidades de $-35<\mathrm{nT}<$ 18 (figura 5.2) lo que lleva a un efecto topográfico que representa sólo una porción del campo total de anomalías magnéticas. Por otro lado, el relevamiento vulcanológico y el muestro paleomagnético no han evidenciado en superficie una variación significativa de la magnetización de las lavas respecto a la media del plateau. Por lo tanto, los cambios locales no se deben a estructuras volcánicas superficiales fuertemente magnetizadas. Además, no se observa correlación de las anomalías de la figura 5.2 con la estructura topográfica del volcán de suaves pendientes. Por estas razones no hay necesidad de tener en cuenta el efecto topográfico magnético.

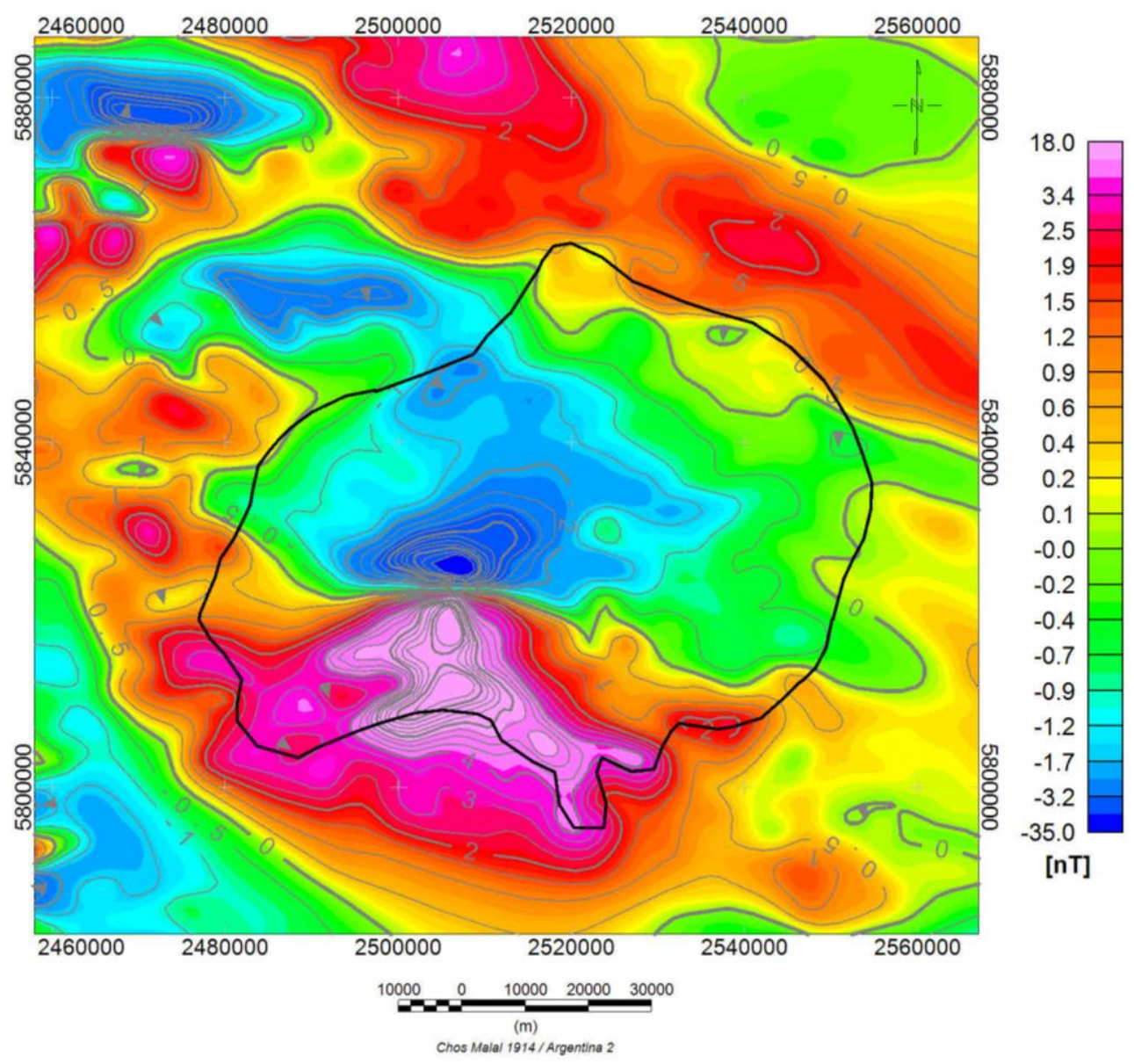

Figura 5.2. Efecto topográfico producido por una placa cortical uniformemente magnetizada de $2 \mathrm{~A} / \mathrm{m}$ en un campo de polaridad reversa $\left(I=35^{\circ}, D=190^{\circ}\right)$. 


\subsubsection{Continuación analítica de campos}

Los filtros de continuación descendente y ascendente son ampliamente usados en el análisis de anomalías magnéticas.

El proceso de continuación descendente consiste en prolongar los datos observados hasta un plano más cercano a las fuentes, siempre que no existan fuentes en la región de continuación. Este cálculo tiende a acentuar el detalle de la distribución de fuentes, especialmente las componentes más superficiales. El problema que presenta este procedimiento es que amplifica las componentes de longitud de onda corta de los datos, por lo cual los hace más "ruidosos", al revés de lo que ocurre con la continuación ascendente, que tiene un efecto de "suavizado" (Blakely, 1995).

La continuación ascendente transforma el campo potencial medido en una superficie a un campo que sería medido en otra superficie más alejada de todas las fuentes. Esta transformación tiende a acentuar las anomalías causadas por fuentes profundas (longitudes de onda larga) a expensas de las anomalías causadas por fuentes someras (longitudes de onda corta), por lo cual este proceso distorsiona los datos medidos. Dependiendo de la profundidad de las fuentes, el área del relevamiento debe ser lo suficientemente grande como para incluir las longitudes de onda largas del espectro de anomalías (Hinze et al., 2013).

Una aplicación de la continuación ascendente es cuando se trabaja con varios relevamientos aéreos medidos en distintos planos y se desea unirlos o compararlos. De este modo, se lleva cada uno de estos relevamientos a un mismo plano. Por otro lado, un relevamiento sobre un terreno volcánico, puede ser dominado por anomalías de longitudes de onda cortas debido a las rocas volcánicas cercanas a la superficie; entonces la continuación analítica puede atenuar esas anomalías con el fin de acentuar las intermedias y más profundas. En consecuencia, este método puede considerarse para atenuar el efecto topográfico.

Siguiendo a Blakely (1995), la transformación ascendente es posible de realizar porque el potencial $U$ cumple con la tercera identidad de Green. Si una función armónica $U$ es continua y tiene derivadas continuas en toda una región regular $R$, entonces la tercera identidad de Green garantiza que el valor de $U$ en cualquier punto $P$ dentro de $\mathrm{R}$ se puede escribir de la forma:

$$
U(P)=\frac{1}{4 \pi} \int_{S}\left(\frac{1}{r} \frac{\partial U}{\partial n}-U \frac{\partial}{\partial n} \frac{1}{r}\right)
$$

donde $S$ denota la superficie que limita la región $R, n$ es la dirección normal hacia fuera de la superficie y $r$ la distancia desde $\mathrm{P}$ al punto de integración sobre S. La ecuación (5.6) expresa que un campo potencial puede calcularse en cualquier punto dentro de una región, a partir del comportamiento del campo sobre la superficie que encierra a dicha región.

El potencial $U$ es en el caso de estudio, el potencial asociado al campo de anomalías, o 
sea un potencial anómalo, $-\nabla U \cdot v_{p}=\Delta T$, donde $v_{p}$ es el versor del campo magnético principal del lugar.

La forma más simple de continuación ascendente es la prolongación de un plano a otro. Supongamos que el campo potencial se midió en una superficie $z=z_{0}$ y se desea conocer el campo en un punto $\mathrm{P}\left(x, y, z_{0}-\Delta_{\mathrm{z}}\right)$ sobre esa superficie, donde $\Delta_{\mathrm{z}}>0$ y el eje $z$ es positivo hacia abajo en el sistema de coordenadas cartesianas. Partiendo de la ecuación (5.6), Henderson (1970) obtuvo la expresión de la integral de la continuación ascendente para un punto $P$ sobre una superficie a partir del conocimiento del campo sobre el nivel original de medición de los datos.

$$
U\left(x, y, z_{0}-\Delta z\right)=\frac{\Delta z}{2 \pi} \int_{-\infty}^{\infty} \int_{-\infty}^{\infty} \frac{U\left(x^{\prime}, y^{\prime}, z_{0}\right)}{\left[\left(x-x^{\prime}\right)^{2}+\left(y-y^{\prime}\right)^{2}+\Delta z\right]^{3 / 2}} d x^{\prime} d y^{\prime}
$$

La ecuación (5.7) es una convolución bidimensional que se puede reescribir como sigue:

$$
U\left(x, y, z_{0}-\Delta z\right)=\int_{-\infty}^{\infty} \int_{-\infty}^{\infty} U\left(x^{\prime}, y^{\prime}, z_{0}\right) \psi_{p}\left(x-x^{\prime}, y-y^{\prime}, \Delta z\right) d x^{\prime} d y^{\prime}
$$

siendo

$$
\psi_{u}(x, y, \Delta z)=\frac{\Delta z}{2 \pi} \frac{1}{\left[x^{2}+y^{2}+\Delta z\right]^{3 / 2}}
$$

Por lo tanto, la representación de la ecuación (5.8) en el dominio de Fourier está dada por:

$$
\mathcal{F}\left[U_{u}\right]=\mathcal{F}[U] \mathcal{F}\left[\psi_{u}\right]
$$

donde $\mathcal{F}\left[U_{u}\right]$ es la transfromada de Fourier del campo continuado hacia arriba y $\mathcal{F}\left[\psi_{u}\right]$ y resulta:

$$
\mathcal{F}\left[\psi_{u}\right]=e^{-\Delta_{z}|k|}, \quad \Delta \mathrm{z}>0
$$

donde $k$ es el número de onda radial en el plano $x y$.

Por lo tanto, la prolongación ascendente de un plano a otro, se obtiene haciendo la transformada de Fourier de los datos medidos o de interés mutiplicando por el factor (5.10) y antitransformando el producto.

En el caso del volcán Auca Mahuida, los datos fueron medidos a altura cuasi-constante (relevamiento barométrico) por lo cual la prolongación de un plano a otro es la más 
adecuada.

La continuación descendente es la operación inversa de la ecuación (5.7) ya que se realiza desde $U\left(x, y, z_{0}-\Delta z\right)$ hasta $U\left(x, y, z_{0}\right)$. Cambios pequeños en $U\left(x, y, z_{0}-\Delta z\right)$ pueden causar variaciones poco realistas en $U\left(x, y, z_{0}\right)$ calculado. En el dominio de Fourier, se representa por la inversa de la ecuación (5.8), donde $\mathcal{F}\left[\psi_{u}\right]=e^{+\Delta_{z}|k|}$. Las longitudes de onda más cortas de los datos medidos serán amplificados hasta un grado que depende de $\Delta \mathrm{z} y$ del intervalo de muestro.

Otra forma de continuación ascendente es la prolongación entre superficies irregulares. Este es el caso cuando los datos adquiridos se disponen en una superficie irregular, por ejemplo en relevamientos aéreos en donde se sigue la topografía. Dos de los métodos más extensamente usados en este caso son el de fuentes equivalentes y aproximación por series de Taylor. Para un detalle pormenorizado ver Blakely (1995).

\subsubsection{Reducción al polo}

Debido al carácter dipolar del campo de anomalías magnéticas producidas por fuentes del subsuelo, los máximos y mínimos de éstas resultan desplazados respecto a las posiciones de las fuentes, y la anomalía se torna asimétrica y con menor amplitud. Esto no ocurre con los máximos y mínimos de los campos de gravedad, debidos a variaciones de densidad, los cuales se sitúan exactamente por encima de las fuentes. Ese corrimiento de los máximos en las anomalías magnéticas complica su interpretación, especialmente a latitudes geomagnéticas bajas. Para contrarrestar este efecto, Baranov (1957) sugirió un método para transformar anomalías magnéticas observadas en cualquier latitud en anomalías que se observarían en presencia de magnetización y campo magnético verticales (Hinze et al., 2013). El método llamado reducción al polo (RTP por sus siglas en inglés), se basa en la relación de Poisson y es uno de los algoritmos más usados en el procesamiento de datos magnéticos. El proceso involucra una transformación lineal de la anomalía observada en el dominio del espacio o del número de onda. Los efectos asociados a la inclinación del campo terrestre con la latitud son removidos, y cada anomalía dipolar se transforma en su contraparte negativa o positiva ubicada directamente encima de la fuente. La figura 5.3 muestra un ejemplo sencillo de una anomalía producida por una línea de dipolos con inclinación magnética de $-30^{\circ}$. Se observa un decrecimiento en la amplitud y un corrimiento frente a la anomalía reducida al polo mostrada en la misma figura. 


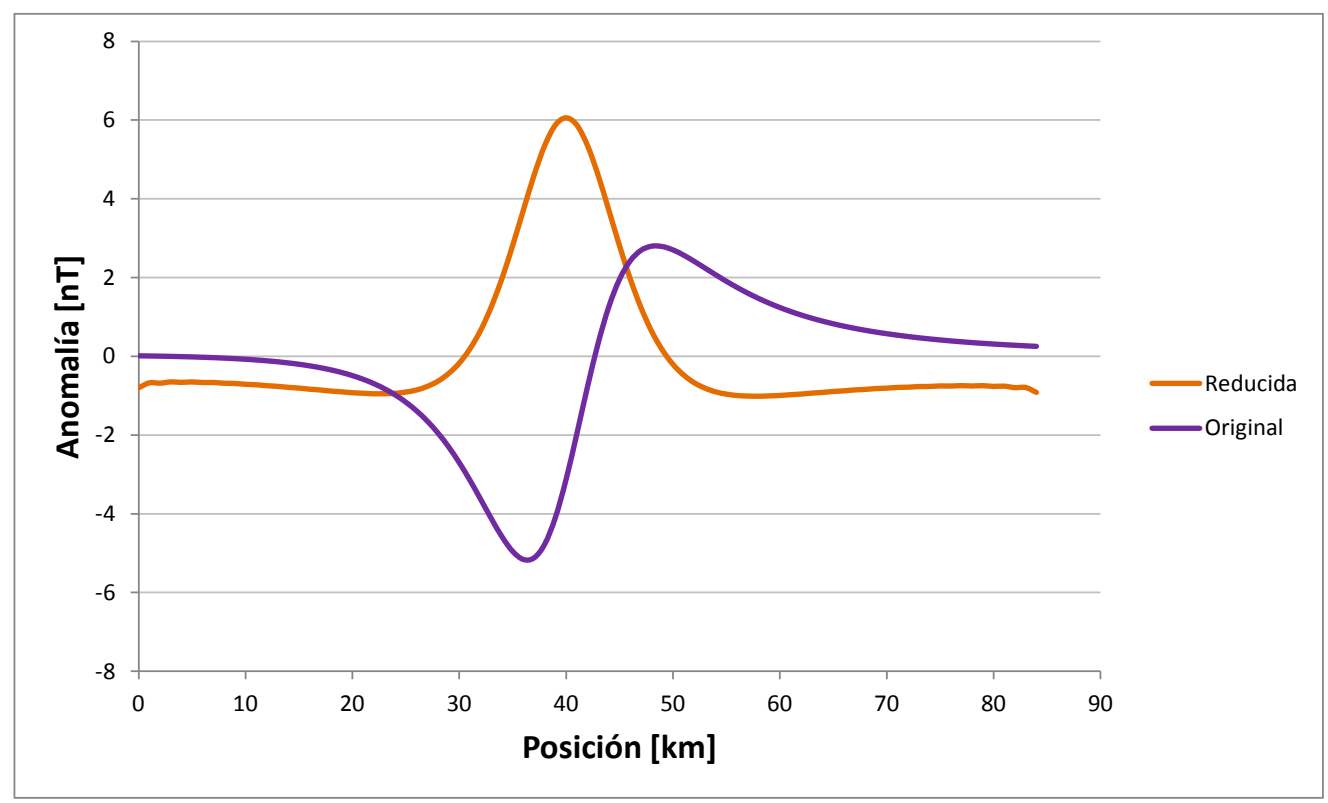

Figura 5.3. Anomalía magnética sintética (original y reducida al polo) producida por una línea de dipolos, ubicada en $x_{0}=40, z_{0}=10 \mathrm{~km}$. El campo ambiente es de $26000 \mathrm{nT}$ y la susceptibilidad magnética de 0.012 (SI). La declinación e inclinación magnética son $0^{\circ} \mathrm{y}-30^{\circ}$, respectivamente y el acimut $=0^{\circ}$. El eje horizontal representa la posición en la dirección perpendicular a la línea de dipolos y el eje vertical corresponde a la magnitud de las anomalías, dado en nT.

Las ecuaciones (5.11) y (5.12) describen la operación de filtrado que transforma el campo total de anomalías con direcciones de magnetización y campo ambiente dadas, en una nueva anomalía causada por la misma distribución de magnetización pero con dirección en la vertical (Blakely, 1995).

$$
\begin{gathered}
\mathcal{F}\left[\Delta T_{r}\right]=\mathcal{F}[\Delta T] \mathcal{F}\left[\Delta \psi_{r}\right] \\
\mathcal{F}\left[\Delta \psi_{r}\right]=\frac{1}{\Theta_{m} \Theta_{f}} \\
\Theta_{m}=\hat{m}_{z}+i \frac{\hat{m}_{x} k_{x}+\hat{m}_{y} k_{y}}{|k|} \\
\Theta_{f}=\hat{f}_{z}+i \frac{\hat{f}_{x} k_{x}+\hat{f}_{y} k_{y}}{|k|}
\end{gathered}
$$

donde $\widehat{\boldsymbol{m}}=\left(\widehat{m}_{x}, \widehat{m}_{y}, \widehat{m}_{z}\right)$ y $\hat{\boldsymbol{f}}=\left(\widehat{f}_{x}, \widehat{f}_{y}, \widehat{f}_{z}\right)$ son los vectores unitarios en la dirección de la magnetización y del campo magnético ambiente, respectivamente. 
La aplicación de $\mathcal{F}[\Delta \psi]$ se conoce como reducción al polo. $\Delta T_{r}$ es la anomalía que sería medida en el polo magnético norte o sur según corresponda, cuando tanto la magnetización inducida y campo ambiente serían verticales.

Una limitación de la RTP, es que el método se vuelve inestable a bajas latitudes, especialmente dentro de los $\pm 15^{\circ}$ del ecuador magnético. Esto resulta en la amplificación del ruido y una distorsión de las anomalías en la dirección del meridiano magnético. Otra limitación es el requerimiento de conocer a priori la dirección de magnetización y la del campo terrestre en el área de estudio, los cuales se suponen constantes en amplitud y dirección, promediados para toda la región en estudio. La aproximación de campo y magnetización constantes es grosera, pero razonable si el área de investigación tiene una extensión geográfica no muy grande (del orden de 100 km x 100 km; Blanco, 1997). Cuando se está en presencia de fuerte remanencia, una transformación RTP correcta requiere tener en cuenta ambas contribuciones, la magnetización inducida y la remanente.

En el caso del volcán Auca Mahuida, la actividad volcánica tuvo lugar durante los periodos Plioceno y Pleistoceno. El campo magnético terrestre sufrió varias inversiones en ese lapso de tiempo y, tal como se señaló antes, el campo de anomalía del volcán muestra principalmente fuentes de magnetización reversa (figura 5.4). Esto significa que la mayor parte de los cuerpos fuente se magnetizaron en un cron inverso y que la remanencia es la contribución magnética dominante en las rocas del VAM (Longo et al., 2015). En consecuencia deben elegirse valores significativos para estos parámetros. Se tomaron valores representativos de la magnetización remanente, acordes con estudios paleomagnéticos previos $^{1}$. Además, para acotar mejor la inclinación se eligió un valor consistente con la variación de inclinación con latitud del Dipolo Axial Geocéntrico (GAD) (Mc Elhinny, 1973) para el área de estudio. En consecuencia, se calculó el campo reducido al polo para dos casos separados, uno considerando que la magnetización de las fuentes es solamente inducida (con valores angulares $I=-38^{\circ}$ y $D=3^{\circ}$, figura 5.5 a y el otro usando la suma vectorial de la inducción y la remanencia (figura 5.5 b). Tal como se argumentó antes, se consideró que la remanencia fue adquirida en un cron de campo terrestre inverso con inclinación y declinación de $38^{\circ}$ y $177^{\circ}$ respectivamente.

El campo reducido al polo calculado considerando sólo la componente de inducción de la magnetización muestra que el algoritmo no es capaz de transformar correctamente las anomalías de magnetización directa (tales como los rasgos I y M en la figura 5.5 a), ya que su posición no resulta alterada. En cambio, las anomalías J, K, L son correctamente desplazadas quedando encima de su fuente, aunque se transforman en rasgos monopolares negativos y por lo tanto con signo cambiado respecto al campo de anomalías TMI. Esto significa que el campo de anomalías está fuertemente afectado por remanencia. El campo reducido al polo obtenido considerando magnetización inducida y remanente muestra que la transformación tampoco remueve totalmente el carácter dipolar, pero la polaridad de las anomalías es

1 Propiedades magnéticas de los basaltos del área del Auca Mahuida, Laboratorio de Paleomagnetismo 'Daniel Valencio', Universidad de Buenos Aires, (datos sin publicar). 
exactamente opuesta a la del campo de la figura 5.5 a. Por lo tanto, los campos reducidos al polo obtenidos con y sin remanencia son muy similares, pero de signos opuestos. De hecho, la remanencia se relaciona con un campo inverso que simplemente invierte la polaridad y no cambia sustancialmente la configuración del patrón magnético.

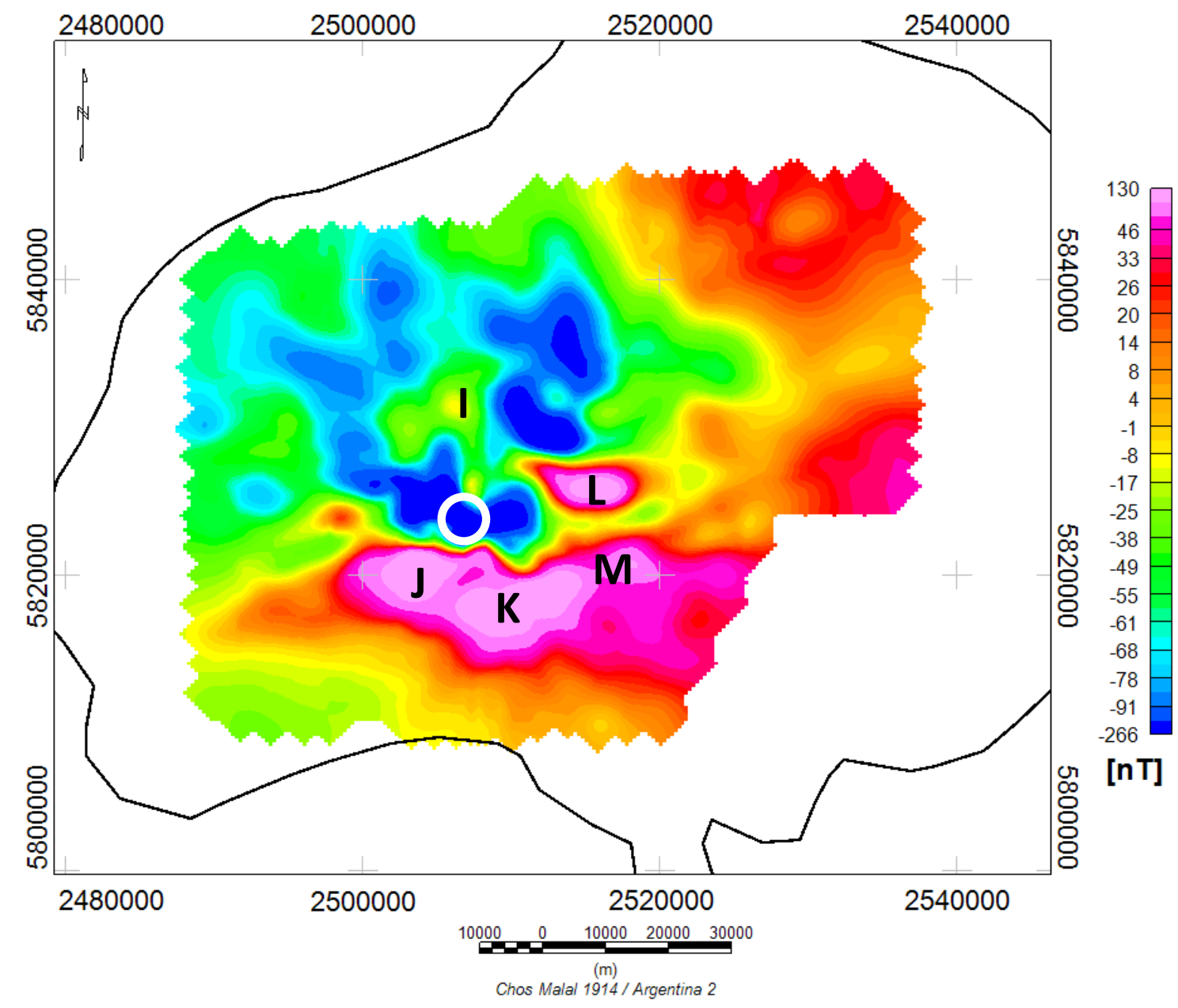

Figura 5.4. Mapa de anomalía magnética del volcán Auca Mahuida. El círculo blanco marca el cráter central. Límite del plateau volcánico es indicado en línea negra. Las letras I, J, K, L, y M especifican las anomalías discutidas en el texto. El relevamiento aeromagnético no cubre todo el plateau. 
a
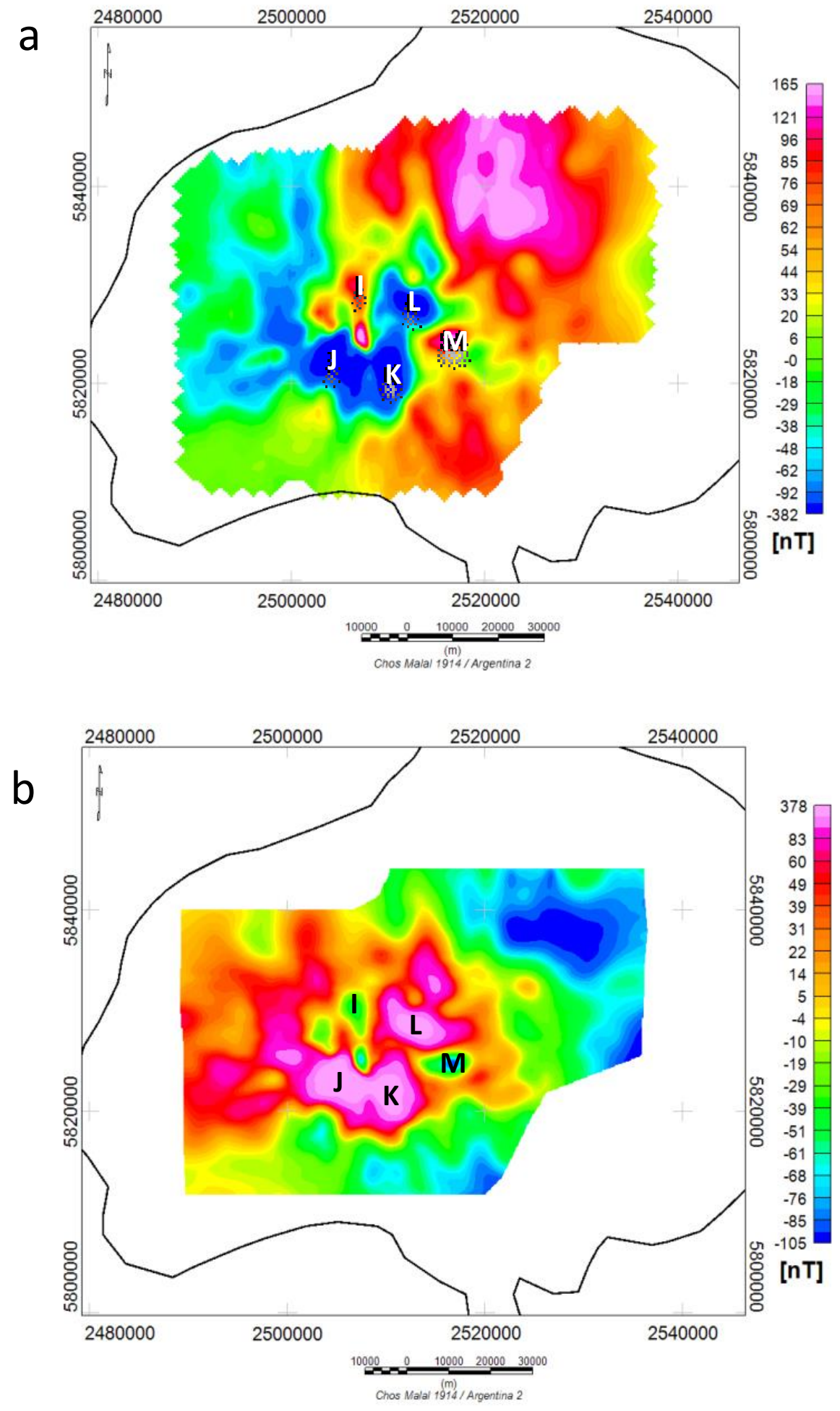

Figura 5.5. a) Transformación RTP calculada considerando solamente la componente de inducción $\left(I=-38^{\circ}\right.$ and $\left.D=3^{\circ}\right)$. b) Transformación RPT calculada con ambas componentes de magnetización, de inducción y remanente $\left(I=38^{\circ}\right.$ and $\left.D=177^{\circ}\right)$. Las anomalías están etiquetadas con letras en color blanco y negro respectivamente (ver Figura 5.4 para su comparación). 


\subsubsection{Señal Analítica}

Se define la señal analítica de una función $f(x)$ como:

$$
a(x)=f(x)-i F_{I}(x)
$$

donde $F_{I}(x)$ es la transformada de Hilbert de la función $f(x)$, dada por:

$$
F_{I}(x)=-\frac{1}{\pi} \int_{-\infty}^{+\infty} \frac{f\left(x^{\prime}\right)}{x-x^{\prime}} d x^{\prime}
$$

La integral (5.14) equivale a la convolución de $f(x)$ con $-1 / \pi x$. Por lo cual, la transformada de Fourier de $F_{I}(x)$ es:

$$
\mathcal{F}\left[F_{I}\right]=i \operatorname{sgn} k \mathcal{F}[f]
$$

La transformada de Hilbert no modifica la amplitud de $f(x)$ pero produce un corrimiento de la fase de $f(x)$ en $\pi / 2$ para $k>0$ y en $-\pi / 2$ para $k<0$.

La transformada de Fourier de la señal analítica entonces se expresa como:

$$
\mathcal{F}[a]=\mathcal{F}[f](1+\operatorname{sgn} k)
$$

Por lo tanto la SA de una función puede calcularse fácilmente a partir de la transformada de Fourier de $f(x)$ como se deduce de la ecuación (5.16); o calculando la transformada de Hilbert de $f(x)$ y sumándole $f(x)$.

El cálculo de la señal analítica (SA) fue introducido en métodos potenciales por Nabighian $(1972,1984)$ como una herramienta para estimar la ubicación y profundidad de un contacto magnético bidimensional.

Roest et al. (1992) propusieron la siguiente expresión para la señal analítica del campo de anomalías:

$$
A(x, y, z)=\frac{\partial \Delta T}{\partial x} \hat{\imath}+\frac{\partial \Delta T}{\partial y} \hat{\jmath}+i \frac{\partial \Delta T}{\partial z} \hat{k}
$$

donde $\hat{\imath}, \hat{\jmath}, \hat{k}$ son los vectores unitarios en la dirección de $x, y, z$, respectivamente. $\Delta T(x, y, z)$ es la anomalía de intensidad magnética total medida sobre un plano. Esta función posee la propiedad necesaria de la función analítica, a saber, sus partes real e imaginaria forman un par transformado de Hilbert. Esto se deduce de que la transformada de la derivada horizontal y vertical están dadas por la ecuación (5.18) (Blakely, 1995). 


$$
\mathcal{F}[\nabla \varnothing]=\boldsymbol{k} \mathcal{F}[\varnothing]
$$

donde $\boldsymbol{k}=\left(\mathrm{i} k_{x}, \mathrm{i} k_{y},|k|\right)$. 0 sea para la anomalía de campo total podemos escribir:

$$
\begin{gathered}
\mathcal{F}\left[\frac{\partial \Delta T}{\partial x}\right]=i k_{x} \mathcal{F}[\Delta T], \quad \mathcal{F}\left[\frac{\partial \Delta T}{\partial y}\right]=i k_{y} \mathcal{F}[\Delta T] \\
\mathcal{F}\left[\frac{\partial \Delta T}{\partial z}\right]=|k| \mathcal{F}[\Delta T]
\end{gathered}
$$

En consecuencia $\frac{\partial \Delta T}{\partial x}, \frac{\partial \Delta T}{\partial y} \mathrm{y} \frac{\partial \Delta T}{\partial z}$ son un par transformado de Hilbert.

La amplitud de SA, $A(x, y, z)$ viene dada por:

$$
|A(x, y, z)|=\left[(\partial \Delta T / \partial x)^{2}+(\partial \Delta T / \partial y)^{2}+(\partial \Delta T / \partial z)^{2}\right]^{1 / 2}
$$

De esta expresión se puede ver que la SA es una medida directa del gradiente del campo magnético, el cual depende de la distancia a la fuente magnética y de la intensidad de magnetización. Como regla general se cumple entonces que a menor distancia de la fuente y mayor intensidad del campo, mayor será la amplitud de la SA.

La señal analítica tiene la ventaja de ser independiente de la dirección de la magnetización y de la dirección del campo magnético terrestre (Blakely, 1995). Esta característica es especialmente conveniente en presencia de remanencia fuerte o en latitudes magnéticas bajas. MacLeod et al. (1993) propusieron que la SA es una alternativa a la reducción al polo para bajas latitudes. Su independencia de la dirección de magnetización implica que todos los cuerpos que tienen la misma geometría tienen la misma señal analítica. Nabighian (1972) mostró que los máximos de la SA están ubicados encima de contrastes magnéticos, de modo que pueden ser usados para identificar los principales contrastes. Dado que los máximos de la señal analítica son simétricos y tienen lugar directamente sobre los bordes de los cuerpos anchos y sobre el centro de los cuerpos estrechos, la interpretación de mapas de señal analítica debería dar indicios sobre la geometría de las fuentes magnéticas (Gunn, 1997).

Nabighian (1972) usó la amplitud de la SA para estimar la profundidad del contacto magnético. Roest et al., (1992) y Atchuta Rao et al., (1981) usaron el ancho de la anomalía a la mitad de la amplitud para derivar las profundidades. Sin embargo, la amplitud medida es usualmente el resultado de muchas anomalías superpuestas, por lo cual, aún cuando la amplitud esté bien definida para una fuente individual, la profundidad resultante tendrá un error considerable si no se usa el modelo correcto. En consecuencia, no se ha usado esta técnica para estimar profundidades dado que el mapa de anomalías del VAM es el resultado de un conjunto de anomalías pequeñas, pero se utilizó para la localización espacial de las 
mismas.

Para obtener resultados confiables, es necesario que los datos tengan buena resolución ya que el ruido se amplifica al derivar. A fin de determinar los principales contrastes de magnetización en el mapa de anomalías del área de estudio, se realizó una prolongación ascendente a $50 \mathrm{~m}$ para suavizar los datos de entrada, y luego se calculó la amplitud de la señal analítica (figura 5.6). En general, su forma resulta similar a la de la anomalía reducida al polo calculado con el efecto de la remanencia (figura 5.5 b).

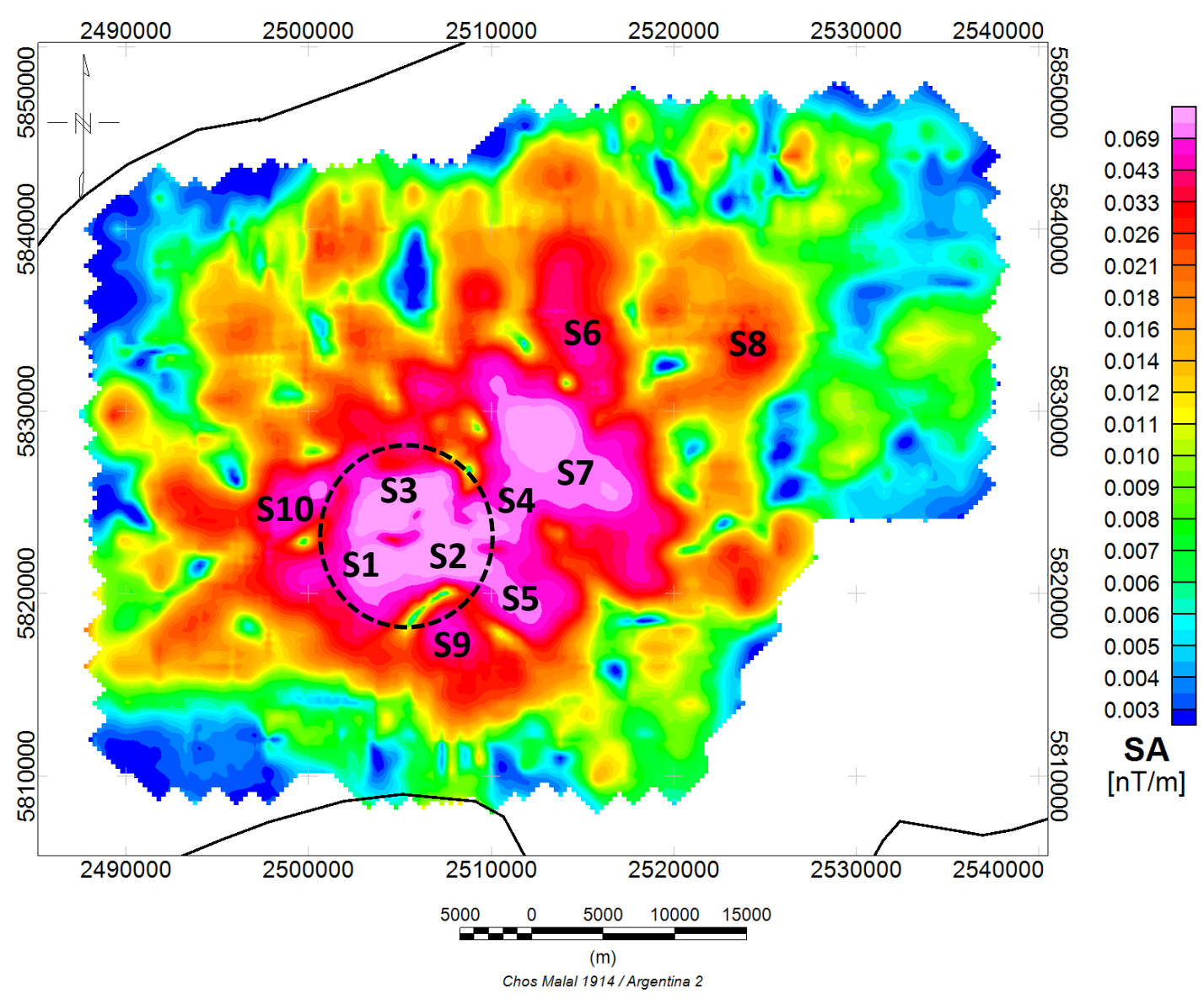

Figura 5.6. Mapa de amplitud de la señal analítica obtenido de la anomalía TMI prolongada hacia arriba $50 \mathrm{~m}$. El círculo de línea discontinua señala el cráter central del volcán. S1 a S10 son las fuentes principales causantes de los máximos de amplitud. La unidad de la amplitud de la SA es $[\mathrm{nT} / \mathrm{m}]$.

En la figura 5.6 los máximos de magnetización S1, S2, S3 se disponen alrededor del cráter central del volcán en forma de anillo, mientras que S4, S5, S6, S7, S9 se orientan según la dirección NO-SE, lo cual sugiere una correlación entre las fracturas existentes en el aparato volcánico y los cuerpos ígneos intruidos. 


\subsubsection{Métodos de localización y profundidad de fuentes magnéticas}

Los métodos de estimación de profundidad a las fuentes magnéticas y gravimétricas están diseñados para tratar anomalías individuales aisladas, mientras que otros analizan muchas anomalías complejas en forma de perfiles o mapas. Estos métodos se basan en técnicas gráficas o en algoritmos computacionales. Las técnicas gráficas, permiten hacer estimaciones de profundidad a las fuentes basadas en la distancia horizontal entre dos puntos de un perfil de anomalías, determinados por ejemplo donde la amplitud es la mitad de su valor máximo, o por el intervalo donde el perfil se aproxima mejor a una recta en su zona de máxima pendiente (Blakely, 1995; Hinze et al., 2013). Estos métodos en general se aplican a cuerpos aislados. Entre los algoritmos de cálculo se encuentran la deconvolución de Euler, deconvolución de Werner, método de señal analítica, técnicas estadísticas de análisis espectral, entre otros. La selección del método dependerá de la experiencia del intérprete, el objetivo del relevamiento, la calidad de las mediciones, la naturaleza del ruido y las anomalías regionales que alteran las anomalías de interés. A pesar de que en teoría el método magnético se puede usar para determinar la profundidad a cualquier fuente magnética aislada que produzca una anomalía observable, se lo aplica más comúnmente a la determinación de profundidad al tope de fuentes intra-basamento. La determinación magnética de profundidades, especialmente cuando se hace con técnicas simplificadas, debe ser corroborada usando más de una técnica. Resultados consistentes no garantizan precisión, pero aumentan la confianza en las profundidades calculadas.

A continuación se hará una descripción de los métodos de localización de las fuentes aplicados a los datos magnéticos de esta tesis cuyos resultados son, como veremos más adelante, validados por los modelos.

\subsubsection{Deconvolución de Euler}

En las últimas décadas se han desarrollado varias técnicas para localizar contactos magnéticos, y una de las más difundidas es la deconvolución de Euler que consiste en la resolución del problema inverso no lineal (Blanco, 1997). Este procedimiento fue descripto por Thompson (1982) para perfiles, y más tarde considerado, con algunas variaciones, por otros autores como Reid et al. 1990 (quienes expandieron la técnica a relevamientos magnéticos bidimensionales), Barbosa et al. 1999, 2000, Mushayandebvu et al. 2001, Hsu 2002, entre otros. Esta técnica consiste en la resolución de la ecuación homogénea de Euler para diferentes ventanas de los datos cubriendo el mapa de anomalías magnéticas completo. Permite obtener la profundidad y la posición horizontal de fuentes magnéticas equivalentes simples. Este método funciona bien para fuentes simples, tales como un dipolo magnético, una línea de dipolos, un monopolo, etc. Las fuentes reales, sin embargo, no son puntuales sino cuerpos extendidos equivalentes a conjuntos de dipolos. 
Una función $f(x, \mathrm{y}, \mathrm{z})$ se dice homogénea de grado n si satisface la expresión:

$$
f(t x, t y, t z)=t^{n} f(x, y, z)
$$

A partir de (5.21), se puede mostrar que se cumple la ecuación conocida como Ecuación de Euler (5.22):

$$
x \frac{\partial f}{\partial x}+y \frac{\partial f}{\partial y}+z \frac{\partial f}{\partial z}=n f
$$

Puede probarse que si $f$ satisface la ecuación de Euler de grado n, entonces la derivada espacial de $f$ es también homogénea y de grado $\mathrm{n}+1$.

Para fuentes magnéticas simples, ubicadas en $\left(x_{0}, y_{0}, z_{0}\right)$, puede mostrarse que el campo de intensidad total $T$ en un punto de observación $\left(x_{j}, y_{j}, z_{j}\right)$, satisface la siguiente ecuación diferencial (5.23):

$$
\begin{aligned}
& \left(x_{j}-x_{0}\right) \frac{\partial B}{\partial x}\left(x_{j}, y_{j}, z_{j}\right)+\left(y_{j}-y_{0}\right) \frac{\partial B}{\partial y}\left(x_{j}, y_{j} z_{j}\right)+\left(z_{j}-z_{0}\right) \frac{\partial B}{\partial z}\left(x_{j}, y_{j} z_{j}\right)= \\
& =-N\left(B\left(x_{j}, y_{j} z_{j}\right)-F\right)
\end{aligned}
$$

donde el parámetro $N$ se conoce como índice estructural, ya que depende del tipo de fuente y está asociado con el grado de homogeneidad del potencial escalar magnético dipolar y su correspondiente campo magnético. Representa la tasa de decaimiento de la anomalía con la distancia a la fuente. Los valores típicos del índice estructural varían entre 0 y 3 dependiendo de la geometría del cuerpo causante de la anomalía. $N$ es el único parámetro que se conoce a priori y es equivalente a -n de la ecuación (5.22).

El escalar $F$ representa el campo ambiente o regional, el cual se asume constante para el conjunto de estaciones consideradas. La remoción de esta componente de los datos magnéticos involucra la supresión del campo geomagnético principal mediante los modelos del IGRF. De esta manera, el campo total $B$ se puede descomponer como $B=\Delta T+F$, siendo $\Delta T$ la anomalía de intensidad total asociada con la fuente magnética concentrada.

La implementación de este algoritmo consiste en la resolución de las ecuaciones de Euler por medio de la inversión de mínimos cuadrados de un conjunto de datos para un índice $N$ dado. Para el caso de un relevamiento distribuido en una malla, esta técnica se aplica seleccionando primero una ventana cuadrada de datos dentro del mapa de anomalía o campo total y calculando sus derivadas ortogonales. Se resuelve la ecuación de Euler, y se obtienen las soluciones $\left(x_{0}, y_{0}, z_{0}\right)$ y su error. Luego se mueve la ventana al siguiente punto de la grilla. Las soluciones cuyo error en la profundidad (desviación estándar) es menor que una 
tolerancia definida (típicamente del 15\%) y que están dentro de una distancia límite de la ventana de observación son aceptadas. Esta distancia límite es la distancia máxima desde el centro de la ventana de búsqueda hasta la posición de una fuente solución de la ecuación. El resultado de la deconvolución es más preciso cuando la fuente está centrada en la ventana de búsqueda, por lo cual aquellas fuentes que son soluciones pero están ubicadas lejos del centro de la ventana pueden ser descartadas. Hay que tener en cuenta que la desviación estándar mide solamente cuán bien se ajusta el campo medido a las ecuaciones de Euler para un dado índice. Esto presupone que el índice elegido es correcto para la fuente del campo observado en la ventana de muestreo, y que el mismo está siendo generado sólo por una fuente.

Una ventaja significativa de la ecuación de Euler para datos magnéticos, es que no está afectada por la inclinación, declinación magnética ni por remanencia (Hinze et al., 2013). Estudios de modelización y trabajos teóricos de Reid et al. (1990) han llevado a la conclusión de que la deconvolución de Euler sin aplicar reducción al polo es capaz de reproducir con precisión la ubicación de fuentes magnéticas de distintos rumbos en presencia de un campo no vertical. La desventaja es que no se obtiene información sobre el buzamiento de las fuentes, el cual debe ser por lo tanto estimado por otros métodos, por ejemplo mediante otras técnicas de modelado inverso.

El método de deconvolución de Euler fue aplicado al mapa de anomalías magnéticas del VAM sin hacer reducción al polo, pues como ya se mencionó, la presencia de remanencia en los datos y la ausencia de una única dirección de magnetización, constituyen una limitación en el cálculo de una correcta transformación. Tomando el ancho de las anomalías entre 1.5 y $5 \mathrm{~km}$, un buen estimador para el tamaño de la ventana es de alrededor de $5 \mathrm{~km}$. Se trabajó con dos índices estructurales, $N$, de 1 y 0.5 . El primero se asocia a geometrías de diques y filones que son las formas más probables de las fuentes en el subsuelo del volcán (Comeron, et al., 2002); y el segundo a fallas. La figura 5.7 muestra las soluciones de la aplicación de la deconvolución de Euler para la ventana de $5 \mathrm{~km}$. En el caso de un relevamiento barométrico (altura constante) las soluciones obtenidas mediante el software Oasis Montaj son referidas al nivel medio del mar. Los valores positivos están sobre el nivel del mar y los negativos debajo de éste. La mayoría de las soluciones se ubican principalmente en los límites de las anomalías, con los topes o techos de las fuentes anómalas a profundidades someras entre 500 y 2000 m respecto al nivel medio del mar. Los cálculos se hicieron de acuerdo a lo descripto más arriba (con una tolerancia en profundidad del $15 \%$ ) y luego con una reducción de este valor a $7 \%$ de modo de obtener soluciones más confiables. Además, se acotó la incerteza en el plano $x y$ en $15 \%$. Por lo tanto la profundidad estimada tiene una incerteza menor al 7\% y la ubicación horizontal es correcta dentro de un 15\%. 
a

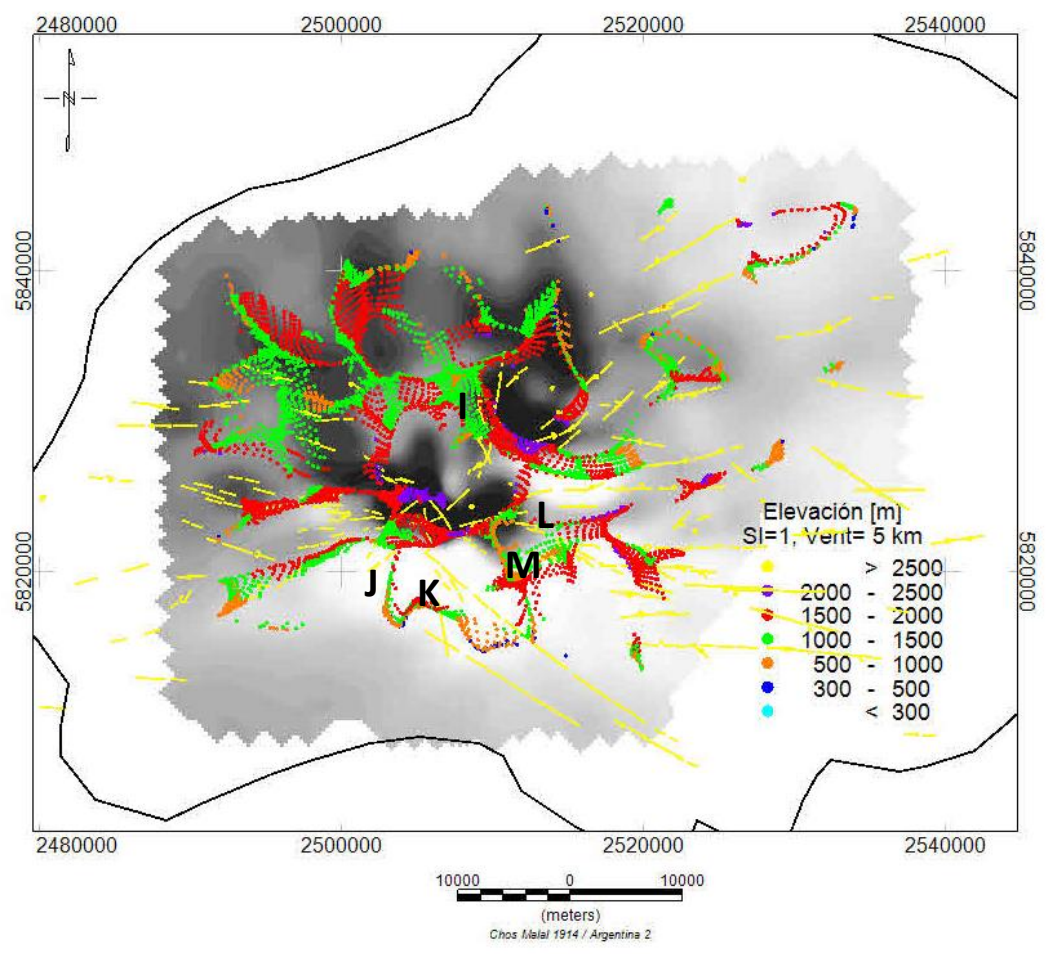

b

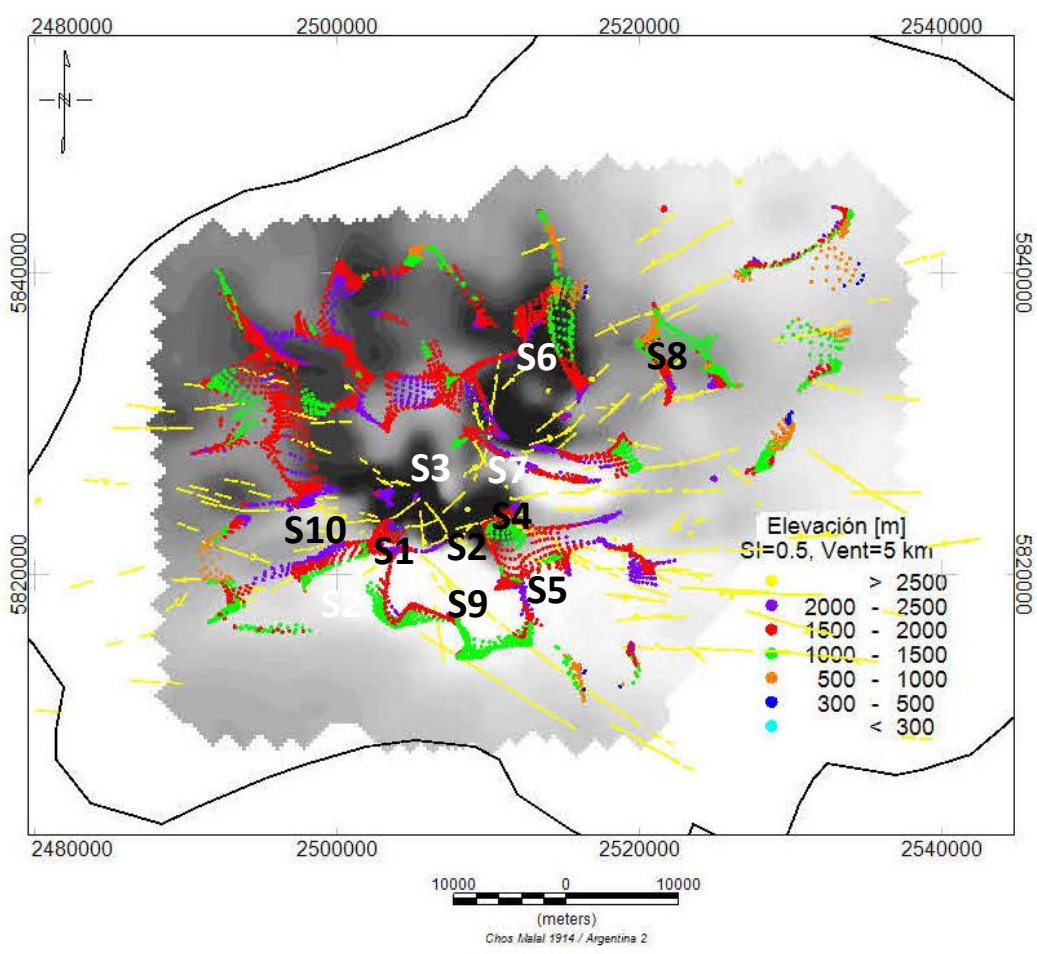

Figura 5.7. Soluciones de deconvolución de Euler obtenidas a partir del mapa de anomalías magnéticas con un tamaño de ventana de $5 \mathrm{~km}$ para un índice estructural dado. a) $N=1$ y b) $N=$ 0.5. La elevación de referencia es el nivel medio del mar dado en metros. En la figura a) I, J, K, M, L son las letras utilizadas en las anomalías de la RTP de la figura 5.5. b) Máximos de amplitud producidos por las principales fuentes en la señal analítica de la figura 5.7 a $(\mathrm{S} 1, \mathrm{S2}, \mathrm{S3}, \mathrm{S4}, \mathrm{S5}$, S6, S7, S8, S9, S10). En trazos amarillos se superpone el patrón de fallas interpretadas en superficie por Del Pino (YPF S. A.). 
Además, se aplicó el método para ventanas de 3, 5 y $10 \mathrm{~km}$ para un SI igual 1 (figuras 5.8 y 5.9$)$.

Como el tamaño de ventana de búsqueda está relacionado al tamaño de celda con que fueron grillados los datos, se regrillaron los datos con 500 y 1000 m para poder tomar ventanas más grandes.

El resultado de la deconvolución tiene la máxima precisión cuando la fuente está centrada en dicha ventana, por lo tanto fuentes ubicadas más allá de una distancia máxima especificada desde el centro de la ventana son rechazadas. En general, las soluciones ubicadas fuera de la ventana de búsqueda pueden ser rechazadas como erróneas.

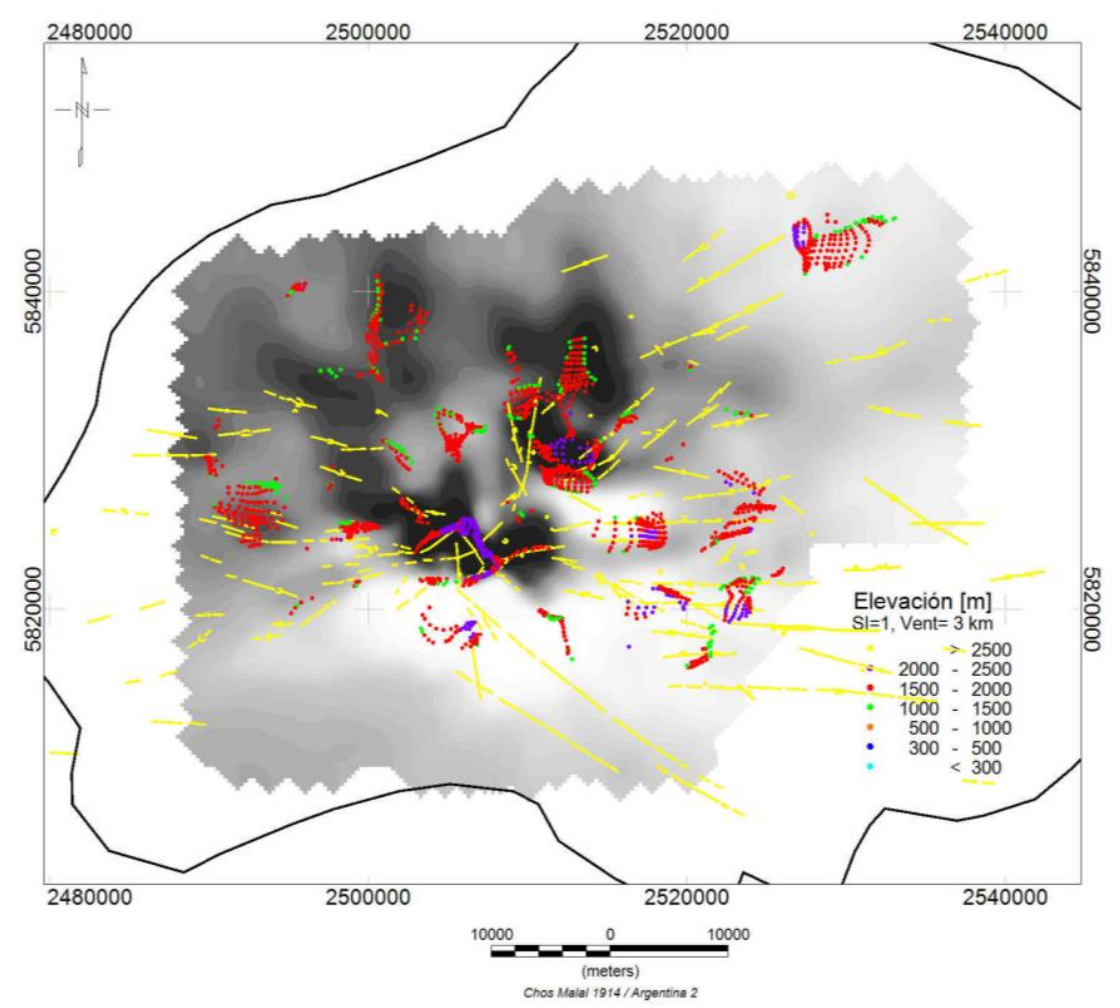

Figura 5.8. Soluciones de Euler obtenidas a partir del mapa de anomalías magnéticas con un tamaño de ventana de $3 \mathbf{~ k m}$ para un índice estructural $N=1$.

La ventana de $3 \mathrm{~km}$ produce una distribución aleatoria de soluciones que no se correlacionan con las mayores intensidades de las anomalías. 
a
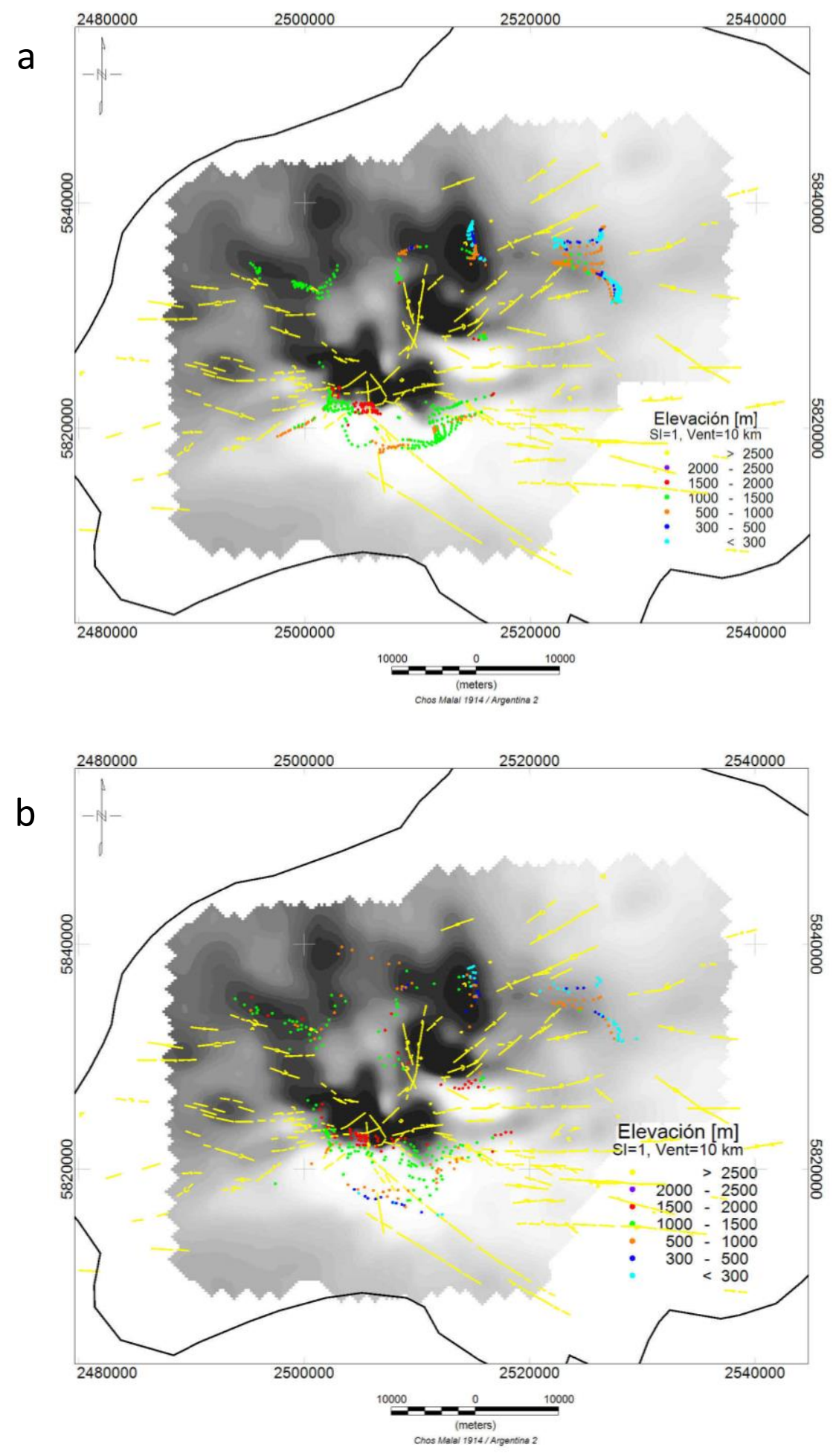

Figura 5.9. Soluciones de Euler obtenidas a partir del mapa de anomalías magnéticas con un tamaño de ventana de $10 \mathrm{~km}$ para un índice estructural $N=1$. a) Tamaño de celda de la grilla $500 \mathrm{~m} \mathrm{y} \mathrm{b)} 1000 \mathrm{~m}$.

La ventana de $10 \mathrm{~km}$ produce pocas soluciones pero con profundidades mayores que las obtenidas con la ventana de $3 \mathrm{~km}$. 
El conjunto de fallas interpretadas en superficie indicadas en color amarillo en la figura 5.8, tiene un diseño radial que confluye en el cráter central. Algunas de las soluciones obtenidas a través del método de Euler se ubican en concordancia con esta distribución. La correlación entre las fuentes magnéticas y el patrón de fallas, refuerza la hipótesis de que parte de estas fracturas pueden ser el resultado de la estructuración asociada a la intrusión de los cuerpos ígneos.

\subsubsection{Profundidad media de un conjunto de fuentes}

La dificultad de aislar unas anomalías de otras, conduce a la utilización de técnicas estadísticas para la localización de los cuerpos magnéticos. La implementación de la estimación del promedio de la profundidad de un gran número de fuentes magnéticas a partir de sus propiedades estadísticas, fue introducida por Spector y Grant (1970). Este método se basa en la expresión del espectro de potencia de la anomalía magnética producido por un conjunto de prismas, los que representan a varias formas geológicas, como diques, venas y filones. Los parámetros que describen a cada prisma (profundidad, espesor, anchura, longitud, magnetización, etc.), obedecen a características similares para todo el conjunto.

Sea $P_{d, t, \ldots}(\delta, \tau, \ldots)$ la probabilidad combinada de que la profundidad $d$ tenga el valor $\delta$, el espesor $t$ tenga el valor $\tau$, y así sucesivamente. Asumiendo que estos parámetros varían en forma independiente unos de otros, la probabilidad conjunta se expresará como producto de las probabilidades individuales de cada variable. Entonces el valor esperado del espectro de densidad de potencia para una anomalía magnética causada por un conjunto de fuentes, viene dado por (Blakely, 1995):

$$
\left\langle P_{\Delta T}\right\rangle=\int_{-\infty}^{+\infty} \ldots \int_{-\infty}^{+\infty}|\mathcal{F}[\Delta \mathrm{T}]|^{2} P_{t}(\tau) P_{d}(\delta) \ldots d \delta d \tau \ldots
$$

donde $|\mathcal{F}[\Delta \mathrm{T}]|^{2}$ es el espectro de la anomalía causada por un solo elemento del conjunto, cuyos parámetros son $\delta$, $\tau$, etc.

Para encontrar una expresión para $|\mathcal{F}[\Delta \mathrm{T}]|^{2}$ procedamos como sigue. Consideremos el campo de anomalía total medida sobre una superficie plana y producida por un paralelepípedo rectangular, con su tope a una profundidad $d$ y espesor $t$, cuyos lados tienen longitudes $2 a$ y $2 b$ en las direcciones $x, y$ respectivamente. Dentro de la sección la magnetización es uniforme $M(x, y)=M_{0}$. En general, la función magnetización $M(x, y)$ no se conoce, pero se tiene alguna idea de cómo ésta se comporta estadísticamente. La aproximación más simple, es asumir que es una función aleatoria de $x$ e $y$. La expresión obtenida por Bhattacharyya (1966) y reescrita por Blakely (1995) es la siguiente: 


$$
|\mathcal{F}[\Delta \mathrm{T}]|^{2}=4 \pi^{2} C_{m}{ }^{2}\left|\theta_{f m}\right|^{2}\left|\theta_{f}\right|^{2} M_{0}^{2} e^{-2|k| d}\left(1-e^{-|k| t}\right)^{2} S^{2}(a, b)
$$

$\theta_{\mathrm{m}}$ y $\theta_{f}$ fueron definidos en la sección $5.3 .3, C_{\mathrm{m}}$ es una constante $(1$ en el sistema emu y $\frac{\mu_{0}}{4 \pi}=10^{-7}$ henry. $\mathrm{m}^{-1}$ en SI) y

$$
S(a, b)=\frac{4 \sin k_{x} a \sin k_{y} b}{k_{x} k_{y}}
$$

es un factor de forma independiente de $d$.

La expresión (5.25) tiene la ventaja de que la transformada de Fourier de la anomalía se puede escribir como el producto de factores separados, a saber, la magnetización y una función que depende de los atributos de la capa (profundidad, espesor, y dirección de magnetización).

Sustituyendo la ecuación (5.25) en la ecuación (5.24) e integrando en cada variable, se obtiene:

$$
\left\langle P_{\Delta T}\right\rangle=4 \pi^{2} C_{m}^{2}\left\langle e^{-2|k| d}\right\rangle\left\langle\left(1-e^{-|k| t}\right)^{2}\right\rangle\left\langle S^{2}(a, b)\right\rangle\left\langle M_{0}^{2}\right\rangle\left\langle\left|\theta_{m}\right|^{2}\right\rangle\left\langle\left|\theta_{f}\right|^{2}\right\rangle
$$

El valor esperado del espectro de densidad de potencia resulta ser el producto de varios promedios sobre colecciones, en el cual cada promedio involucra sólo una de las variables aleatorias.

Es fácil mostrar que si la profundidad al tope de cada prisma varía entre $d$ - $\Delta d$ y $d+$ $\Delta d$ con $\Delta d$ pequeña, resulta:

$$
\left\langle e^{-2|k| d}\right\rangle=e^{-2|k| \bar{d}}
$$

y el logaritmo de este factor como función de $|k|$ es una recta cuya pendiente es $\mathrm{m}=-2 \bar{d}$.

$$
\ln \Delta_{T}=\ln \left(A e^{-2|k| \bar{d}}\right)=\ln (A)-2|k| \bar{d}
$$

Si el número de onda está expresado en unidades de ciclos por unidad de longitud, entonces la profundidad viene dada por $\bar{d}=\mathrm{m} / 4 \pi$, mientras que si la unidad de $\mathrm{k}$ es radianes por unidad de longitud, $\bar{d}=\mathrm{m} / 2$.

El espectro de densidad de energía promediado radialmente es independiente de la dirección de la magnetización de las fuentes y de la dirección del campo geomagnético, o sea es una función del número de onda solamente y se calcula promediando la energía para todas las direcciones para el mismo número de onda. Esta metodología puede usarse para estudiar un amplio rango de profundidades variando la ventana usada en el análisis de los datos. Un 
paso crítico en el procedimiento es la selección del tamaño de la ventana de datos. La profundidad máxima de investigación es de alrededor del 20 al 25\% de la longitud de la ventana, o diámetro en el caso de promedio radial del espectro. Una ventana demasiado pequeña no capturará suficiente número de anomalías como para promediar adecuadamente, y una muy grande promediará las profundidades sobre una distancia demasiado amplia y por lo tanto carecerá de significado geológico (Hinze et al., 2013). Si la grilla es lo suficientemente grande como para incluir muchas fuentes, se puede determinar la profundidad $\bar{d}$ del tope promedio del conjunto de fuentes a partir del espectro logarítmico de esos datos.

Dos limitaciones que presenta esta metodología es que proporciona buenos resultados en estudios a escala regional y en el presente estudio el relevamiento es de escala local, y no considera contrastes laterales de magnetización.

Para un tamaño máximo de ventana de $35 \mathrm{~km}$ x $40 \mathrm{~km}$-dimensiones del mapa de anomalías magnéticas del VAM-, la máxima profundidad que se podría obtener es de 7-8 km. La figura $5.10 \mathrm{~b}$ muestra valores de profundidad estimada calculando la derivada promediada cada 5 puntos del espectro de energía. Se obtiene una profundidad máxima promedio del conjunto de fuentes magnéticas a aproximadamente $3.5 \mathrm{~km}$ medida a partir de la altura de vuelo. El tramo de la pendiente del logaritmo del espectro de potencia relacionado con números de onda bajos, corresponde a las fuentes más profundas, mientras que la recta que ajusta la curva para valores de número de onda más altos, está relacionada con las fuentes más superficiales (figura $5.10 \mathrm{a}$ ). 
a

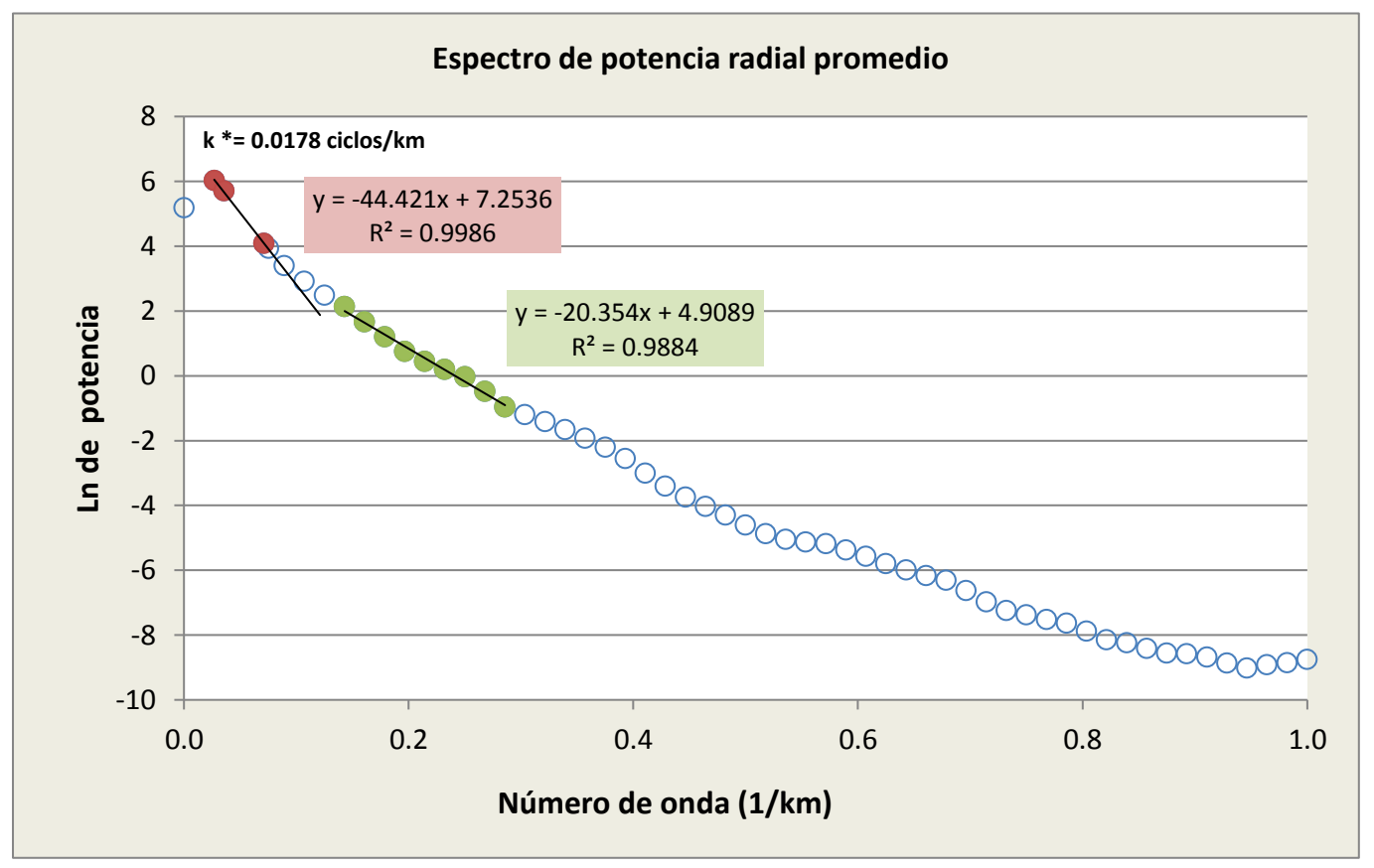

b

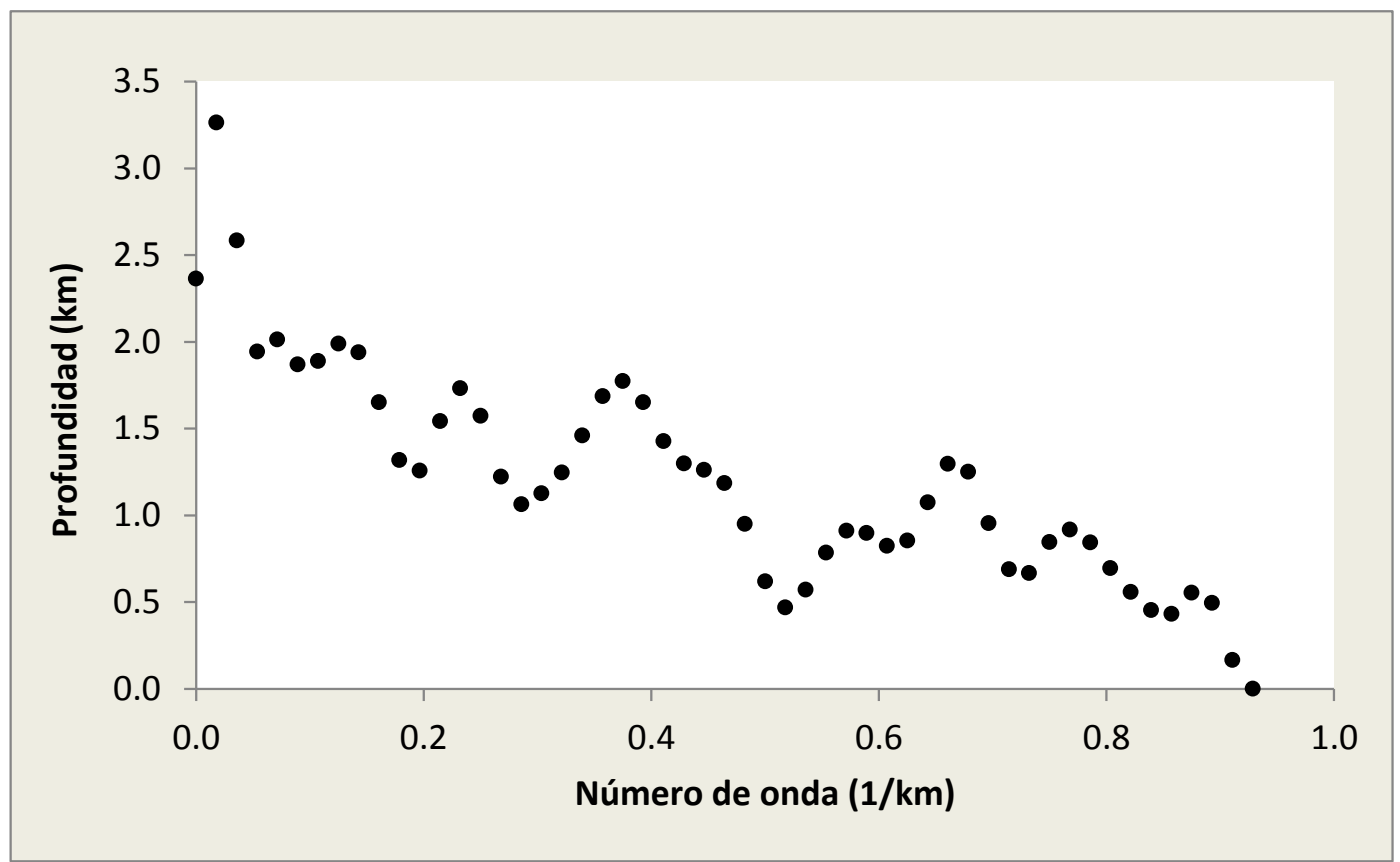

\begin{tabular}{|c|c|c|}
\hline Pendiente & $\begin{array}{c}\text { Prof. Estimada } \\
(\mathrm{km})\end{array}$ & $\begin{array}{c}\text { Rango nro. de } \\
\text { onda }\end{array}$ \\
\hline-44.42 & 3.53 & $0.027-0.071$ \\
\hline-20.35 & 1.62 & $0.143-0.286$ \\
\hline
\end{tabular}

Figura 5.10. a) Profundidad de las fuentes magnéticas estimada a partir del espectro de potencia logarítmico promediado radialmente del mapa de anomalías. El espectro corresponde a grupos de cuerpos magnéticos con la misma profundidad media. $\mathrm{k}^{*}$ es el número de onda correspondiente al máximo en el espectro de potencia. b) Profundidades estimadas a partir de la pendiente del espectro tomada en intervalos de 5 puntos. 
El error típico del ajuste de la pendiente y, por lo tanto, de estimación de la profundidad viene dado por la expresión 5.29 (Sánchez Jiménez, 2003):

$$
\varepsilon=\frac{\mathrm{m} \sqrt{\frac{1-R^{2}}{n}}}{4 \pi}
$$

donde $\mathrm{m}$ es la pendiente de la recta, $\mathrm{R}$ el coeficiente de correlación y $\mathrm{n}$ el número de puntos en la recta.

Reemplazando los valores obtenidos en la recta de ajuste para las fuentes más profundas, el error de ajuste resulta en 0.076 .

\subsection{Campo de anomalías regionales}

Los datos aeromagnéticos regionales cubren aproximadamente $115000 \mathrm{~km}^{2}$ (gran parte de la Cuenca Neuquina) y la cantidad de puntos medidos en el área son 6151. Teniendo en cuenta el intervalo de muestreo nominal $S$, ecuación (5.2), se usó un tamaño de celda de 1 $\mathrm{km}$, y se grillaron los datos con el algoritmo de mínima curvatura. Desafortunadamente este relevamiento no cubre el edificio central del volcán Auca Mahuida, de modo que estos datos fueron usados para realizar una caracterización cualitativa de los rasgos magnéticos de longitudes de onda más larga que rodean al relevamiento de 2001, siempre que fueran consistentes con los de este último.

El campo principal de los relevamientos de 1968 y 2001, fue corregido por el IGRF de esos años, respectivamente, por lo que no habría necesidad de realizar la corrección por la variación secular. Por otro lado como los mapas de anomalías son consecuencia de magnetización remanente principalmente, la correlación entre ambos es posible.

Las longitudes de onda de las anomalías de los datos regionales están comprendidas entre 40 y $80 \mathrm{~km}$ y se extienden decenas de kilómetros alrededor del plateau volcánico (figura 5.11 a). Las anomalías R1, R2, R3 y R4 no están totalmente representadas en el relevamiento de 2001, por lo cual no es posible modelarlas con estos datos.

El mapa regional de anomalía magnética muestra que el área del VAM se sitúa entre una zona de valores bajos del campo magnético cortical (sector occidental R4) y una zona de bajo gradiente (sector oriental). Hacia el noreste y sureste se ubican dos lóbulos positivos de longitud de onda larga (R1 y R2) en correspondencia con el levantamiento de rocas del basamento debido a la acción del fallamiento directo de Entre Lomas (Cristallini et al., 2009). Estas observaciones fundamentan que las longitudes de onda corta del campo de anomalías del VAM sean atribuidas a fuentes volcánicas. Si la lava fuese la fuente de las anomalías del campo medido, la intensidad de ésta última sería mucho más alta. Sin embargo, como se analizó anteriormente a través del cálculo del efecto topográfico de una capa de $2 \mathrm{~A} / \mathrm{m}$, las anomalías magnéticas observadas en el relevamiento local no se correlacionan con la 
topografía. Esto implica que las fuentes (intrusiones) se encuentran debajo del manto de lava y por lo tanto son significativas desde el punto de vista interpretativo volcánico-estructural.

a
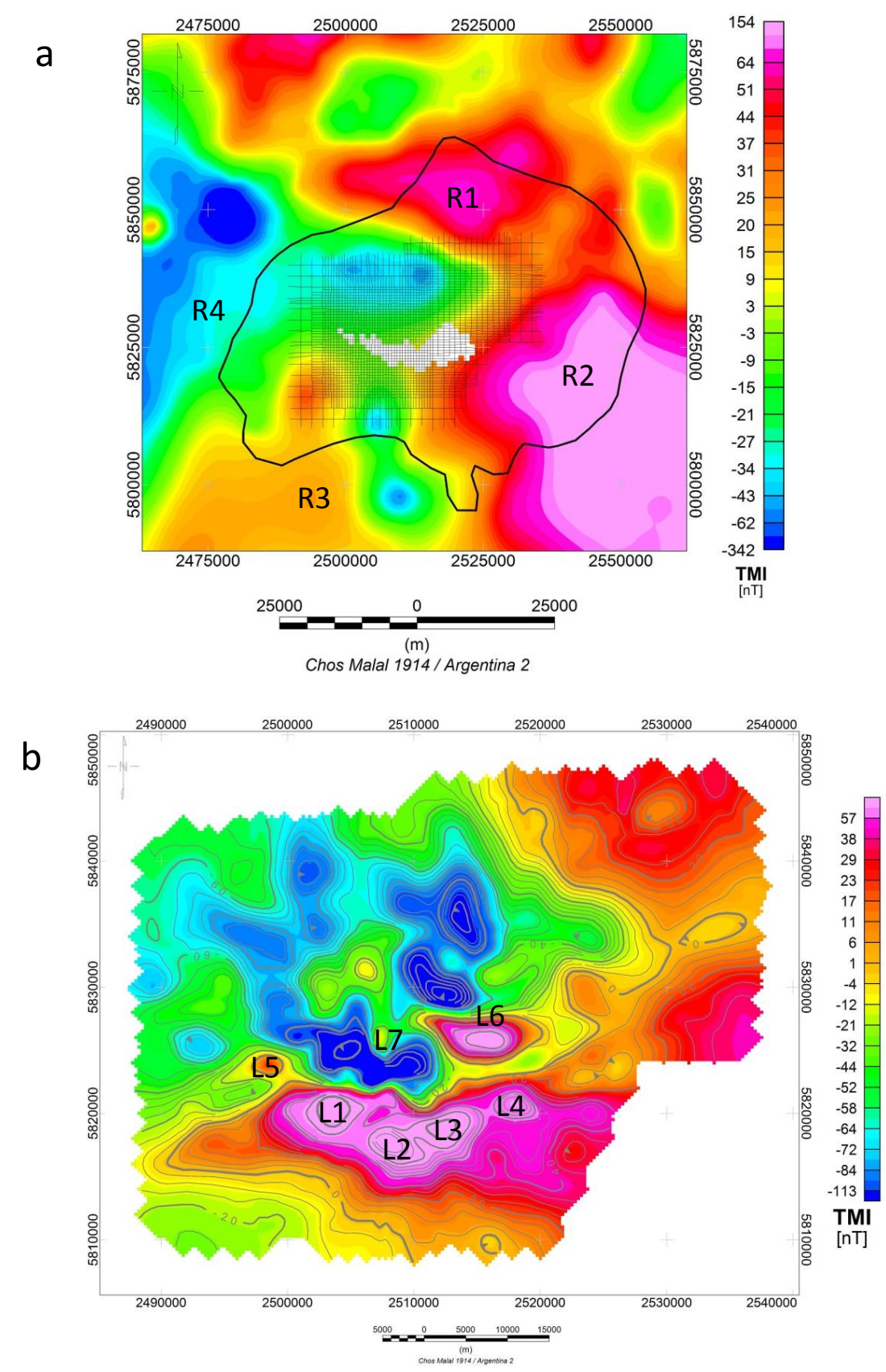

Figura 5.11. a) Relevamiento regional de 1968. R1, R2, R3 y R4 representan a las anomalías de longitudes de onda larga. En línea negra se indica el límite del plateau volcánico y las líneas de vuelo de la adquisición de los datos locales se grafican sobre la grilla. b) Campo de anomalías del relevamiento 2001. L1, L2, L3, L4, L5, L6 y L7 representan a las anomalías individuales principales. La escala TMI está dada en [nT] para ambas figuras. 
En el relevamiento aéreo del VAM (figura 5.11 b) se pueden reconocer dos grupos principales de anomalías (figura 5.11 a). El primer grupo, está formado por anomalías simples compactas de carácter dipolar en la zona central del plateau del volcán (L5, L6 y L7). El segundo, está caracterizado por una secuencia de anomalías amalgamadas en la ladera sur del volcán que forman un cinturón magnético elongado en sentido E-O de $20 \mathrm{~km}$ de longitud (L1, L2, L3, L4). Este último grupo de anomalías no tiene una forma dipolar simple debido a su superposición espacial y a la influencia de anomalías regionales de mayor longitud de onda (R2 y R3).

Como se indica en la figura 5.12, las áreas ubicadas entre la Depresión de Añelo, la estructura volcánica de Cerro Bayo y la región al este de la faja plegada y corrida del Agrio se caracterizan por bajos valores de la anomalía (R4). Este patrón está interrumpido en el sur por un rasgo positivo NE-SO a N-S relacionado con el flanco oriental del alto estructural de Chihuidos (R3). Anomalías positivas de alta intensidad se encuentran al este-sudeste y en la zona del alto de Chihuidos (R3), y al norte del plateau del Auca Mahuida (R1 y R2).

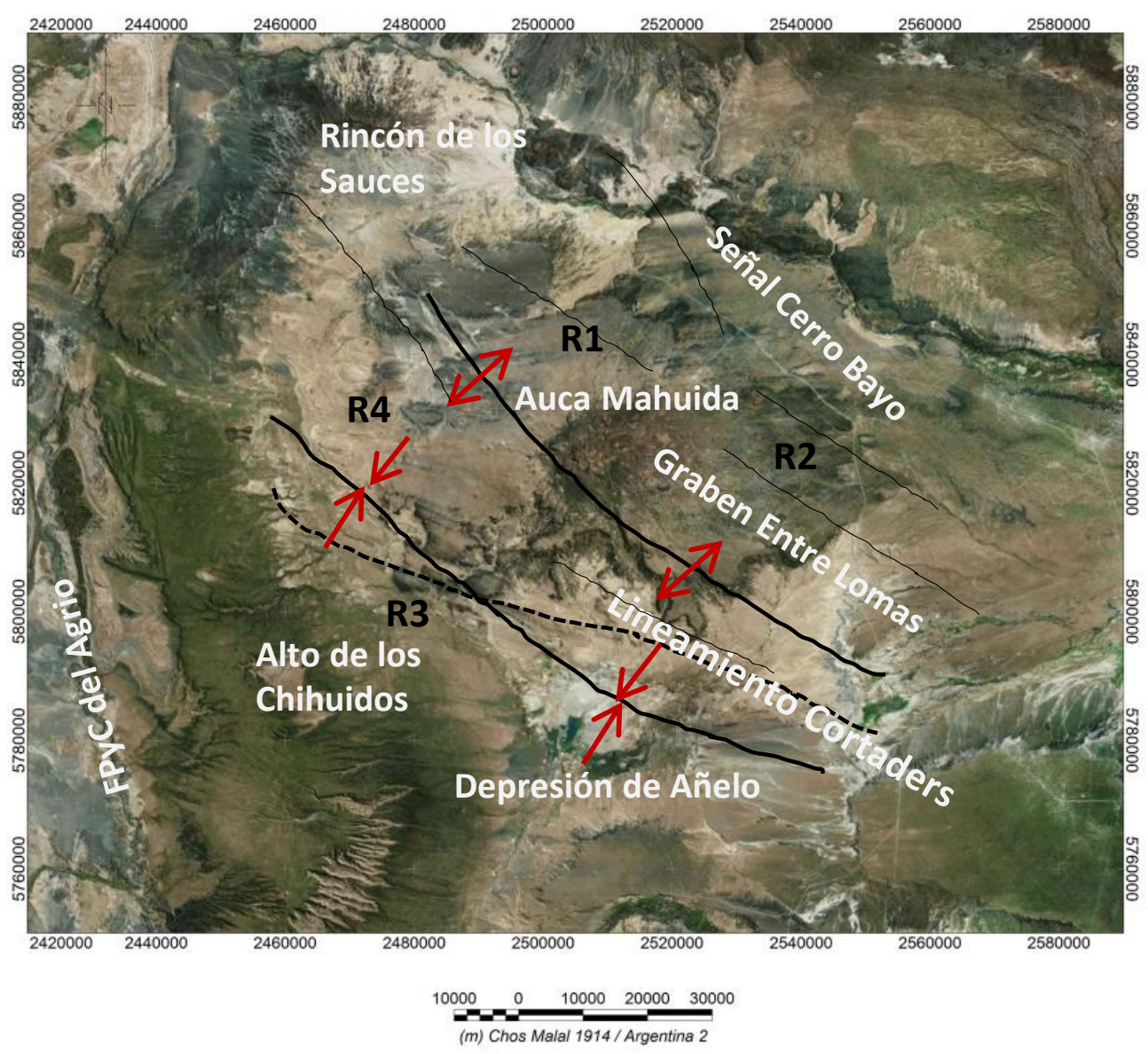

Figura 5.12. Principales rasgos estruturales del complejo volcánico Auca Mahuida. R1, R2, R3 y $\mathbf{R} 4$ correspenden a las anomalías mostradas en los datos aeromagnéticos de la figura 5.11 b). 


\subsection{Interpretación de los resultados}

A continuación se presenta un resumen de los resultados obtenidos en este capítulo.

- El mapa de anomalías magnéticas del volcán Auca Mahuida (figura 5.1) muestra un conjunto de fuentes con una magnetización predominantemente reversa, situado alrededor del cráter central con longitudes de onda en el rango de 1.5 a $5 \mathrm{~km}$.

- Las anomalías magnéticas no presentan correlación con ningún efecto topográfico, como se observa en la figura 5.3, lo cual confirma su origen en profundidad.

- Los campos reducidos al polo obtenidos con y sin remanencia son muy similares, pero de signos opuestos. La remanencia se relaciona con un campo inverso que simplemente invierte la polaridad y no cambia sustancialmente la configuración del patrón magnético.

- En el mapa de amplitud de la señal analítica se reconocen máximos de magnetización alrededor del cráter central del VAM (S1, S2, S3 en la Figura 5.7) como también en el sector oriental, donde se observan rasgos de alta intensidad (S6 y S8) orientados de NO a SE (S4, S5, S7). Esto sugiere una correlación entre las fuentes magnéticas y las fracturas, proveniente de la propia génesis del cuerpo volcánico.

- Las soluciones de la deconvolución de Euler se localizan en los límites de las anomalías, y podrían estar asociadas a estructuras de diques, filones capa y fallas en el subsuelo.

- El análisis espectral radial del campo de anomalías magnéticas del VAM identifica los topes de las fuentes a profundidades someras con un máximo en $3.5 \mathrm{~km}$ bajo la elevación de vuelo (700 m debajo del nivel medio del mar).

- Estos valores son consistentes con las profundidades obtenidas de la deconvolución de Euler, comprendidas entre 500 y 2000 m respecto al nivel medio del mar.

- El mapa regional de anomalías magnéticas pone de manifiesto la superposición e influencia de las anomalías en ambos relevamientos y su relación con el patrón estructural de la zona. 


\section{CAPÍTULO VI}

\section{INTERPRETACIÓN DE LOS DATOS GRAVIMÉTRICOS Y SÍSMICOS}

La complejidad geológica-estructural del área de estudio requiere que los datos gravimétricos y aeromagnéticos sean complementados con el análisis de otros datos geofísicos, tales como sísmica 2D y 3D e información geológica de perforaciones y superficie. De este modo, la integración de los mismos permite reducir la ambigüedad inherente a la interpretación de campos potenciales. Entre los datos adquiridos en la perforación de pozos, los registros de densidad, la velocidad (perfil sónico), rayos gamma, etc., proveen valores de los parámetros utilizados en el procesamiento e interpretación de la gravimetría y la sísmica. Sin bien la calidad del dato sísmico es pobre en la zona central del volcán, en las zonas circundantes del plateau (fuera de los límites del relevamiento aéreo del VAM) mejora la relación señal-ruido, lo que favorece la interpretación de las principales formaciones sedimentarias y los cuerpos ígneos intruidos en esta secuencia.

\subsection{Anomalías gravimétricas del VAM}

Las anomalías gravimétricas nos permiten estudiar y modelar las fuentes presentes en la corteza y manto superior, ya que son las que reflejan variaciones laterales de densidad. Esta contribución es una fracción pequeña de la gravedad observada, del orden de $0.01 \%$ de la aceleración de la gravedad en la superficie de la Tierra. Estas anomalías se obtienen a partir de los datos medidos mediante técnicas de reducción (Capítulo III), de las cuales la de aire libre y de Bouguer son las correcciones más importantes.

Los valores de gravedad correspondientes al relevamiento de alta resolución de 2001 llevado a cabo por Carson Aerogravity, fueron reducidos por aire libre y Bouguer. La base de datos entregada por ellos carece del valor de gravedad medido durante la adquisición. La figura 6.1 representa la grilla de anomalías de aire libre interpolada con el algoritmo de mínima curvatura con un tamaño de celda de $500 \mathrm{~m}$. Este relevamiento (al igual que el aeromagnético) no es lo suficientemente extenso como para cubrir todo el plateau volcánico. Como indica Hinze et al., (2013) el diseño del relevamiento debe basarse en la máxima profundidad de las fuentes de interés y en la amplitud de las anomalías regionales en la zona, de modo de poder aislar los efectos locales de los más profundos que responden a anomalías 
regionales. Estas últimas deben ser mapeadas mediante un relevamiento gravimétrico extendido más allá del área de interés. Una regla práctica común es que la extensión lateral del relevamiento gravimétrico debe ser como mínimo tres veces la profundidad máxima de las fuentes de interés, más allá de los límites del área de interés. Esto se debe a que la anomalía debida a una concentración de masa decrece hasta un $10 \%$ o menos de su máxima amplitud a esa distancia. Esta distancia debe ser considerada un mínimo.

Con el relevamiento de alta resolución gravimétrico, no es posible modelar los espesores de la secuencia sedimentaria los cuales serían sólo visibles con longitudes de onda larga a escala regional.

La anomalía de aire libre tiende a alcanzar valores altos en zonas donde el terreno es elevado, lo cual produce una correlación indeseada entre topografía y gravedad. Esto es evidente en las curvas del perfil de la figura 6.2, donde si bien la corrección de aire libre tuvo en cuenta la variación en elevación de las mediciones, no tuvo en cuenta la masa adicional representada por el edificio topográfico.

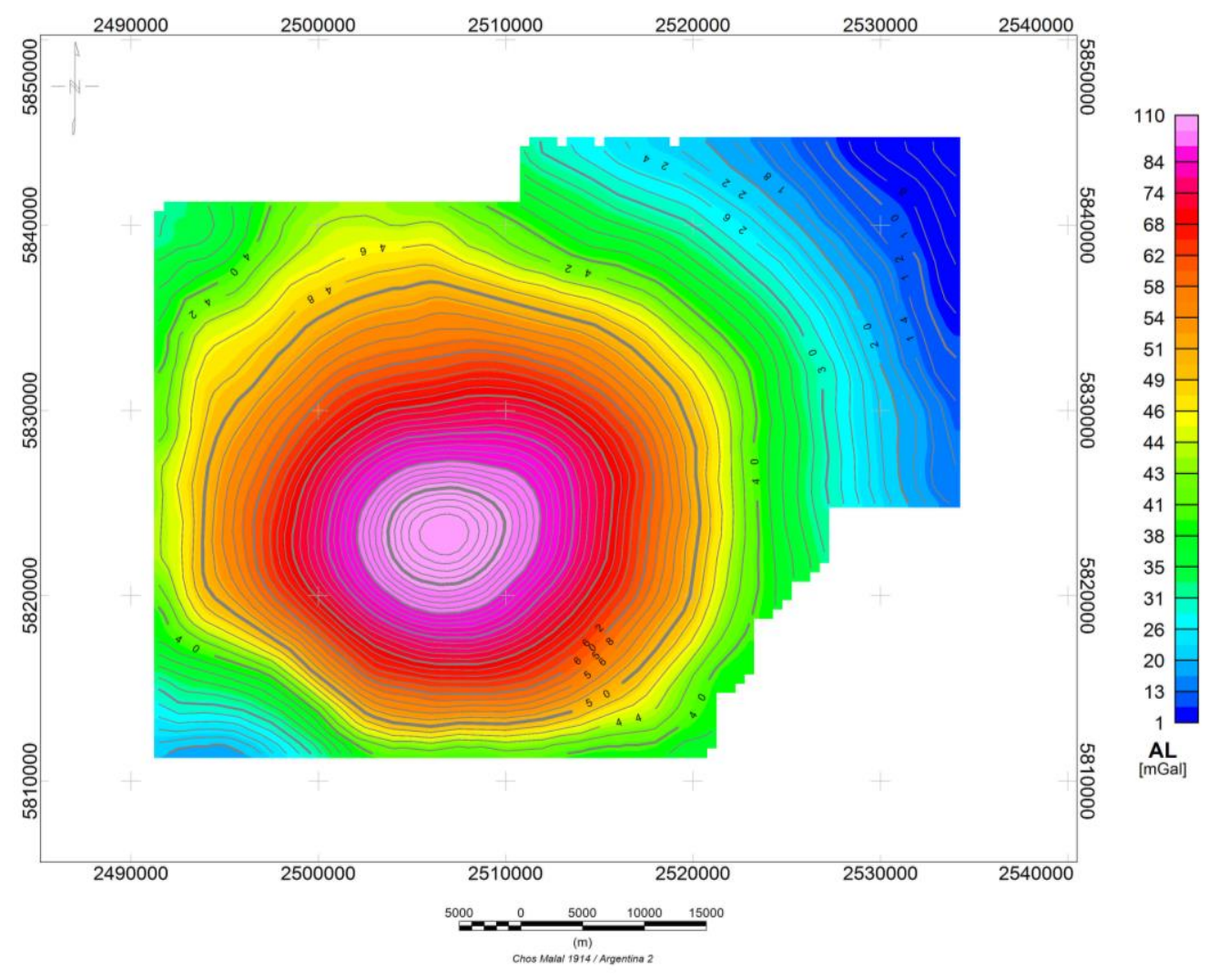

Figura 6.1. Mapa de anomalía de aire libre obtenido por Carson. 
La anomalía de Bouguer de la base de datos de la empresa Carson Aerogravity (figura 6.3) difiere de la obtenida en este trabajo a partir de la anomalía de aire libre, como se verá en la siguiente sección. El hecho de no contar con los datos medidos, limita la posibilidad de corroborar cuál de ellas es la que describe mejor el efecto gravimétrico en el volcán. Suponiendo que la anomalía de aire libre está bien calculada, lo cual se puede sostener por la alta correlación con la topografía, la anomalía de Bouguer calculada en esta tesis es razonable. Por lo expuesto, no se ha utilizado la anomalía de Bouguer de Carson Aerogravity en el modelado.

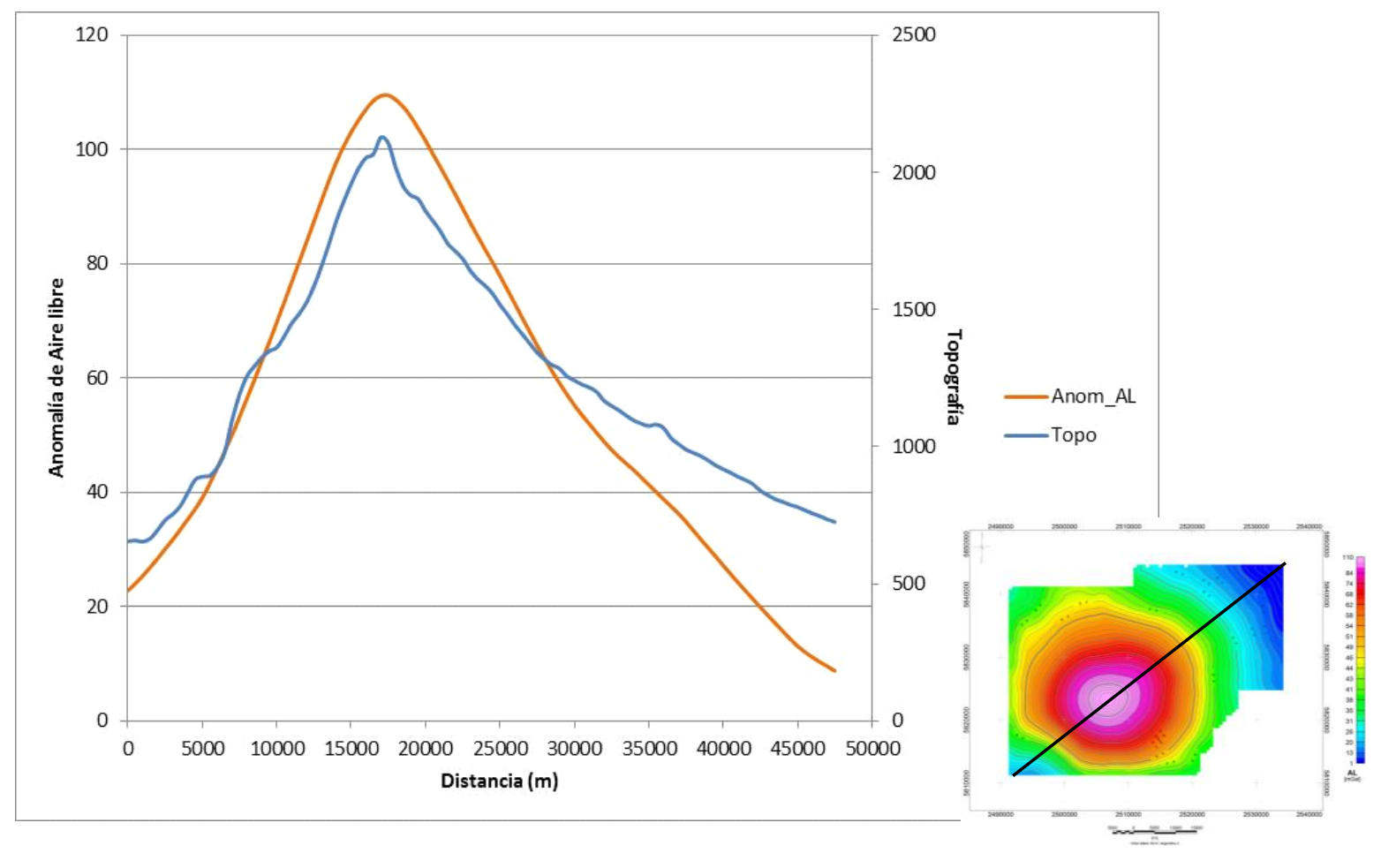

Figura 6.2. Curvas con valores de anomalía de aire libre (naranja) y topografía (celeste) sobre el perfil en línea negra NO-SE graficado sobre el mapa de anomalía. 


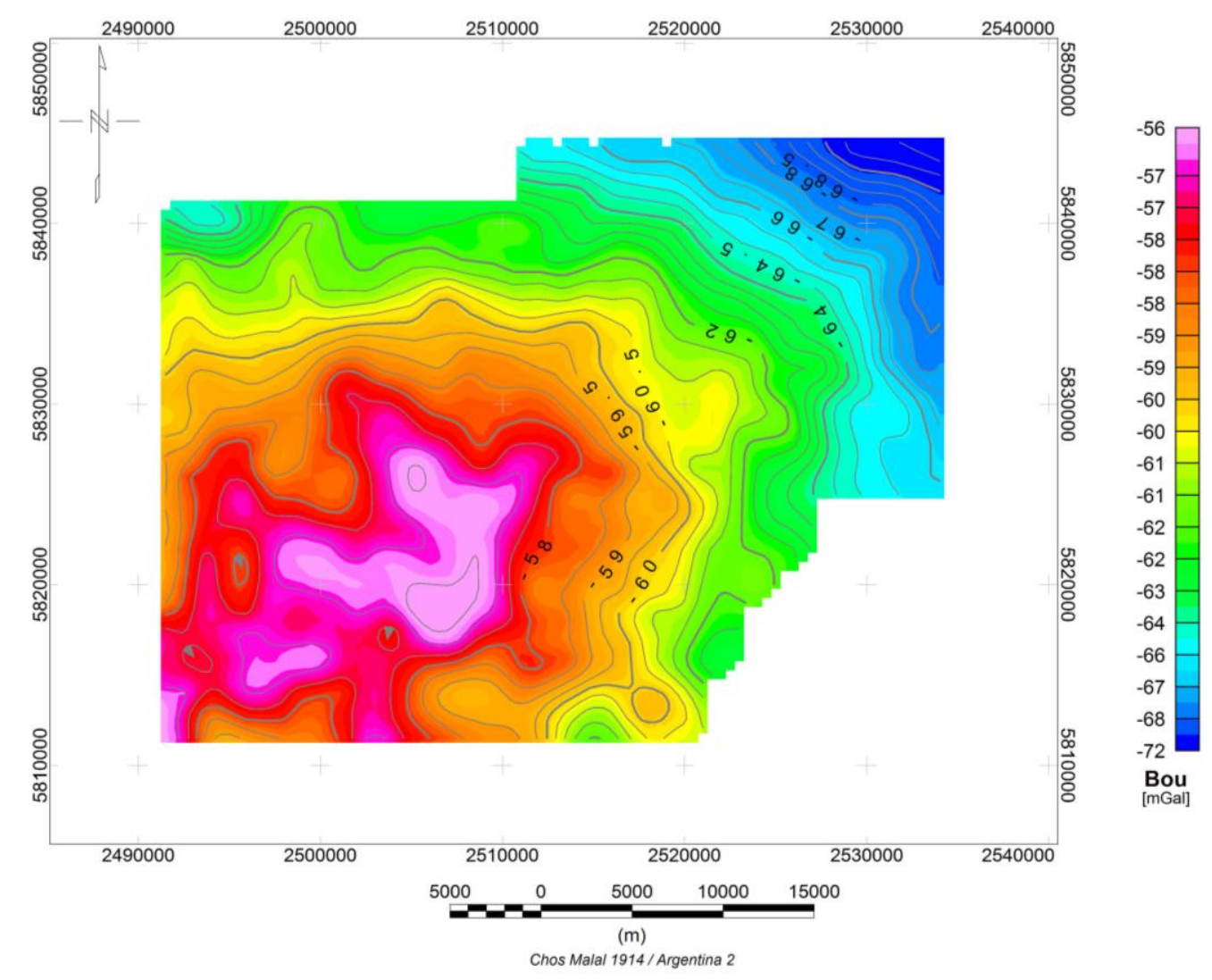

Figura 6.3. Anomalía de Bouguer obtenida por la empresa Carson Aerogravity con una densidad de $2.43 \mathrm{~g} / \mathrm{cm}^{3}$.

\subsubsection{Determinación del valor de densidad topográfica}

La anomalía de Bouguer simple (figura 6.4) tiene en cuenta la densidad de una placa entre la estación de medida y el geoide de referencia. La elección de la densidad en el proceso de reducción es un paso importante. La densidad de corrección se tomó del valor promedio de los registros de densidad obtenidos en 73 pozos de la cubierta sedimentaria. Estos datos son confiables pues provienen de mediciones puntuales. La tabla 6.1 contiene los valores de densidad para cada pozo, donde el valor mostrado es un promedio sobre la columna atravesada.

Un procedimiento muy utilizado para la obtención del valor de densidad es el descripto por Nettleton en 1939. Se seleccionaron tres perfiles sobre la anomalía simple de Bouguer y se graficaron los valores de la anomalía calculada con los distintos valores de densidad pero no se encontró una inversión en estas curvas. Ello se traduce en que no existe curva que tenga una correlación mínima con la topografía. Esto se debe a que las condiciones para la aplicación de esta metodología no se cumplen en el área de estudio, motivo por el cual se descartaron las soluciones obtenidas y se adoptaron los valores de pozos.

La anomalía de Bouguer simple (ABS) se calculó con una densidad de corrección de 
$2.43 \mathrm{~g} / \mathrm{cm}^{3}$ (figura 6.4). Esta reducción se realizó a partir de la anomalía de aire libre calculada por Carson Aerogravity; y la anomalía de Bouguer completa (ABC) se obtuvo mediante la adición de la corrección topográfica (figura 6.5) con la misma densidad.

La determinación de la corrección topográfica de cada estación de gravedad se realizó con el algoritmo de Geosoft ${ }^{\mathrm{TM}}$ que utiliza una combinación del método descripto por Nagy (1966) para la zona cercana e intermedia; y en la zona alejada, el descripto por Kane (1962). Pare ello se utilizó un modelo digital de elevaciones de alta resolución $90 \mathrm{~m}$ de espaciamiento de grilla). Se calcula la $g_{\mathrm{t}}$ en cada punto a partir del efecto gravimétrico de prismas según la expresión de Nagy, 1966. Contrariamente a lo que se espera en general para relevamientos terrestres, corrección topográfica positiva, en relevamientos aéreos barométricos pueden resultar también valores negativos. Esto se debe a que hay zonas donde la diferencia entre la topografía y altura de las estaciones es muy grande (laderas del volcán) y en otras menor (cumbre del volcán). Según Fairhead (2016), las mediciones aéreas son similares a las marinas pero a altura variable sobre el nivel del mar, por lo cual la corrección topográfica puede ser positiva o negativa.

La tabla 6.2 muestra los rangos de las anomalías calculadas.

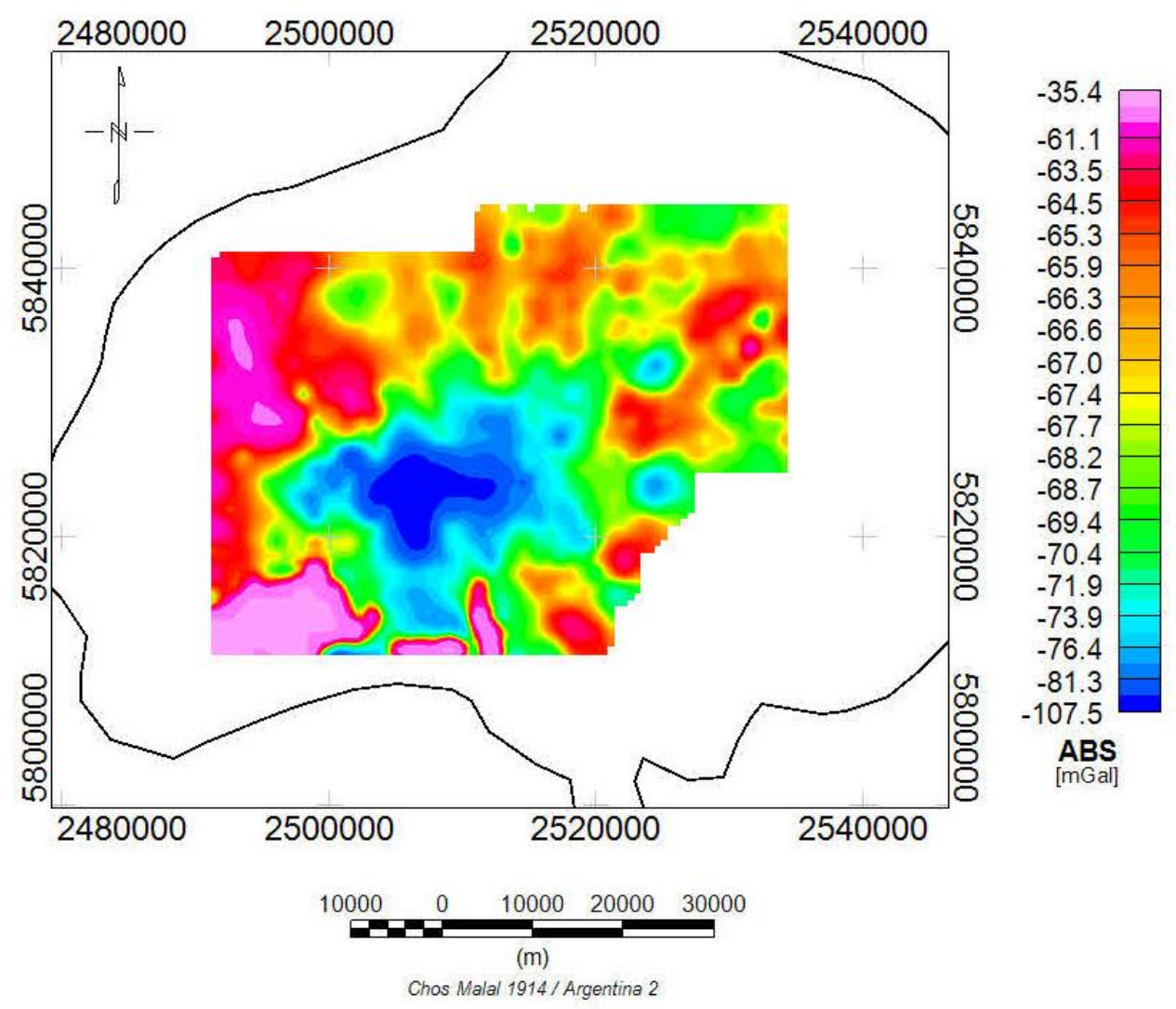

Figura 6.4. Anomalía de Bouguer simple calculada con una densidad de $2.43 \mathrm{~g} / \mathrm{cm}^{3}$. 


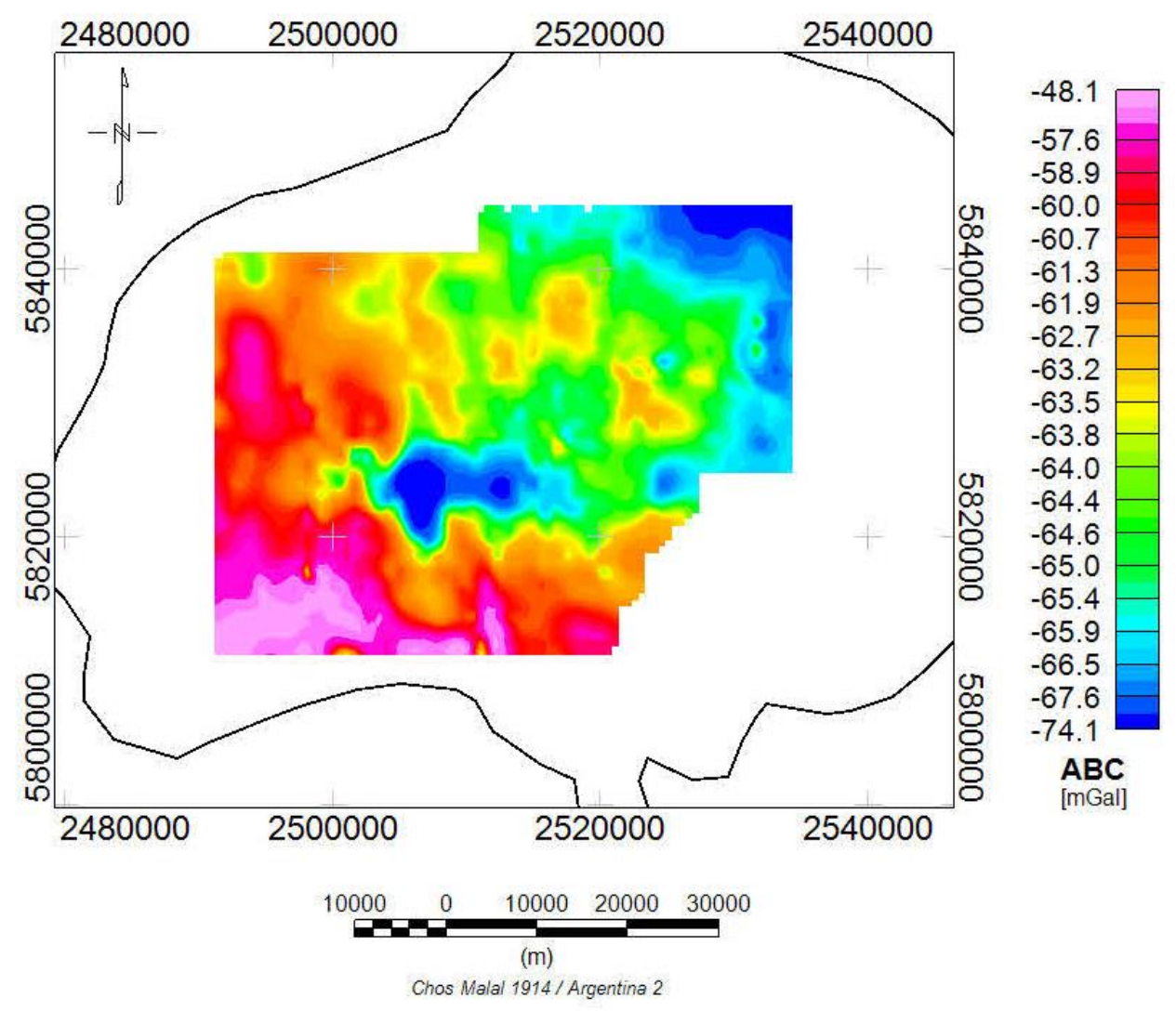

Figura 6.5. Anomalía de Bouguer completa (con corrección topográfica) calculada con una densidad de $2.43 \mathrm{~g} / \mathrm{cm}^{3}$.

La anomalía de Bouguer total del área estudiada (figura 6.5), presenta valores en su totalidad negativos con un mínimo en la zona del cráter, con longitud de onda que alcanza los $14 \mathrm{~km}$. Este mínimo está asociado a rocas ígneas de menor densidad que los basaltos y rocas sedimentarias y a la presencia de gran cantidad de fracturas y fallas de las rocas en el sector del cráter central. Existe una clara correspondencia con los rasgos topográficos, lo que hace suponer que el campo de anomalías responde a fuentes superficiales.

Se observa además un gradiente negativo SO-NE de alrededor de $15 \mathrm{mGal}$ en $40 \mathrm{~km}$ aproximadamente. 
Tabla 6.1. Valores de densidad promedio para cada pozo obtenidos de los perfiles expresado en $\mathrm{g} / \mathrm{cm}^{3}$.

\begin{tabular}{|l|r|r|r|r|r|}
\hline \multicolumn{1}{|c|}{ Pozo } & \multicolumn{1}{c|}{ X } & \multicolumn{1}{c|}{ Z } & Z(+)abajo & den_prom \\
\hline CoBy.x-1ST & 2508109.93 & 5823964.71 & -732.51 & 732.51 & 2.486 \\
\hline EV.x-1 & 2505557.00 & 5832517.00 & -1781.15 & 1781.15 & 2.436 \\
\hline EV.x-2 & 2509144.00 & 5833918.00 & -1167.46 & 1167.46 & 2.390 \\
\hline LaMa-3 & 2507555.52 & 5821658.32 & -778.25 & 778.25 & 2.411 \\
\hline LaMa.x-1 & 2509276.00 & 5820330.00 & -887.84 & 887.84 & 2.383 \\
\hline LaMa.x-2 & 2507893.00 & 5821422.00 & -799.22 & 799.22 & 2.429 \\
\hline LAn.x-1 & 2510960.00 & 5818340.00 & -1801.95 & 1801.95 & 2.352 \\
\hline LRb.x-1 & 2500719.00 & 5824552.00 & -969.16 & 969.16 & 2.397 \\
\hline RiAl-3 & 2505427.69 & 5821929.02 & -745.2 & 745.2 & 2.449 \\
\hline RiAl-7 & 2505666.73 & 5821814.55 & -786.68 & 786.68 & 2.486 \\
\hline RiAI.a-4 & 2506075.97 & 5821467.47 & -790.27 & 790.27 & 2.423 \\
\hline RiAI.x-1 & 2505304.13 & 5821396.41 & -826.48 & 826.48 & 2.419 \\
\hline RiAIN.a-5 & 2505589.98 & 5824649.34 & -771.62 & 771.62 & 2.466 \\
\hline RiAIN.x-1 & 2507472.44 & 5823270.09 & -727.78 & 727.78 & 2.459 \\
\hline RiAIN.x-2 & 2506024.27 & 5825514.42 & -783.06 & 783.06 & 2.454 \\
\hline RiAIN.x-2ST & 2507023.75 & 5826091.87 & -784.17 & 784.17 & 2.415 \\
\hline VAM-13 & 2507065.25 & 5828351.96 & -883.24 & 883.24 & 2.380 \\
\hline VAM-14 & 2509346.63 & 5825909.42 & -821.69 & 821.69 & 2.444 \\
\hline VAM-15 & 2508761.11 & 5826062.34 & -824.17 & 824.17 & 2.413 \\
\hline VAM-16 & 2509576.97 & 5826232.87 & -837.36 & 837.36 & 2.408 \\
\hline VAM-18 & 2508917.52 & 5826651.06 & -839.65 & 839.65 & 2.398 \\
\hline VAM-19 & 2508338.61 & 5826785.30 & -859.65 & 859.65 & 2.398 \\
\hline VAM-20 & 2508052.17 & 5826441.67 & -779.71 & 779.71 & 2.441 \\
\hline VAM-21(I) & 2508611.79 & 5826447.81 & -837.01 & 837.01 & 2.409 \\
\hline VAM-22 & 2508201.97 & 5828186.52 & -874.41 & 874.41 & 2.392 \\
\hline VAM-25 & 2508359.01 & 5828508.91 & -877.11 & 877.11 & 2.392 \\
\hline VAM-26 & 2508547.92 & 5827682.73 & -883.41 & 883.41 & 2.412 \\
\hline VAM-27 & 2508022.62 & 5827149.43 & -859.54 & 859.54 & 2.363 \\
\hline VAM-28ST & 2508057.81 & 5825613.93 & -755.11 & 755.11 & 2.452 \\
\hline VAM-30 & 2509439.81 & 5825506.26 & -817.58 & 817.58 & 2.421 \\
\hline VAM-31 & 2508294.32 & 5825979.57 & -332.05 & 332.05 & 2.404 \\
\hline VAM-32 & 2508361.58 & 5829230.12 & -870.7 & 870.7 & 2.351 \\
\hline VAM-34 & 2507919.90 & 5829104.38 & -873.58 & 873.58 & 2.374 \\
\hline VAM-38 & & -870.08 & 870.08 & 2.380 \\
\hline
\end{tabular}


Tabla 6.1. Continuación. Valores de densidad promedio para cada pozo obtenidos de los perfiles expresado en $\mathrm{g} / \mathrm{cm}^{3}$.

\begin{tabular}{|c|c|c|c|c|c|}
\hline Pozo & $x$ & $Y$ & $Z$ & $\mathrm{Z}(+)$ abajo & den_prom \\
\hline VAM-39 & 2507272.56 & 5827574.59 & -872.2 & 872.2 & 2.412 \\
\hline VAM-40 & 2507759.01 & 5828081.11 & -883.59 & 883.59 & 2.396 \\
\hline VAM-42 & 2508184.36 & 5827745.23 & -873.38 & 873.38 & 2.432 \\
\hline VAM-45 & 2507869.11 & 5828623.23 & -876.71 & 876.71 & 2.384 \\
\hline VAM-46 & 2508942.28 & 5825836.42 & -815.66 & 815.66 & 2.405 \\
\hline VAM-50 & 2508007.17 & 5829472.21 & -878.87 & 878.87 & 2.401 \\
\hline VAM-52 & 2507370.07 & 5826756.10 & -793.79 & 793.79 & 2.416 \\
\hline VAM-55 & 2508612.54 & 5825280.30 & -748.06 & 748.06 & 2.435 \\
\hline VAM-57 & 2507408.36 & 5825921.84 & -333.49 & 333.49 & 2.479 \\
\hline VAM-58 & 2507596.75 & 5826784.15 & -356.86 & 356.86 & 2.400 \\
\hline VAM-59 & 2507159.52 & 5825566.46 & -758.59 & 758.59 & 2.449 \\
\hline VAM-63ST & 2508376.98 & 5828813.57 & -873.1 & 873.1 & 2.391 \\
\hline VAM-65 & 2507848.94 & 5825909.54 & -761.36 & 761.36 & 2.452 \\
\hline VAM-66 & 2508117.82 & 5826210.04 & -341.88 & 341.88 & 2.411 \\
\hline VAM-67 & 2506791.40 & 5826498.06 & -799.02 & 799.02 & 2.426 \\
\hline VAM-69 & 2506698.68 & 5825328.47 & -764.56 & 764.56 & 2.435 \\
\hline VAM-7 & 2507353.00 & 5828242.00 & -880.96 & 880.96 & 2.425 \\
\hline VAM-70 & 2507664.21 & 5825286.60 & -736.46 & 736.46 & 2.429 \\
\hline VAM-73 & 2506500.25 & 5825045.63 & -741.77 & 741.77 & 2.441 \\
\hline VAM-8 & 2507599.44 & 5827294.03 & -822.39 & 822.39 & 2.356 \\
\hline VAM- 80 & 2507637.37 & 5826256.45 & -775.55 & 775.55 & 2.430 \\
\hline VAM.a-10 & 2508587.63 & 5828075.57 & -882.13 & 882.13 & 2.392 \\
\hline VAM.a-11(I) & 2509144.00 & 5826273.00 & -830.55 & 830.55 & 2.397 \\
\hline VAM.a-12 & 2507868.49 & 5830598.88 & -889.35 & 889.35 & 2.363 \\
\hline VAM.a-17 & 2507677.35 & 5825614.13 & -756.76 & 756.76 & 2.421 \\
\hline VAM.a-23ST2 & 2509524.52 & 5824902.18 & -816.18 & 816.18 & 2.433 \\
\hline VAM.a-24 & 2510513.60 & 5825616.45 & -857.73 & 857.73 & 2.384 \\
\hline VAM.a-29 & 2508170.04 & 5829725.13 & -877.98 & 877.98 & 2.370 \\
\hline VAM.a-33 & 2509226.04 & 5829354.03 & -904.62 & 904.62 & 2.396 \\
\hline VAM.a-33ST & 2508801.52 & 5829480.43 & -892.98 & 892.98 & 2.395 \\
\hline VAM.a-36 & 2505696.22 & 5828031.75 & -897.38 & 897.38 & 2.413 \\
\hline VAM.a-4 & 2507388.14 & 5827875.47 & -875.81 & 875.81 & 2.383 \\
\hline VAM.a-47 & 2506728.13 & 5825722.05 & -786.33 & 786.33 & 2.467 \\
\hline VAM.a-6 & 2507204.60 & 5829628.22 & -846.31 & 846.31 & 2.370 \\
\hline VAM.a-6ST & 2507079.47 & 5829649.20 & -882.82 & 882.82 & 2.355 \\
\hline VAM.e-5 & 2508570.89 & 5827045.08 & -870.39 & 870.39 & 2.369 \\
\hline VAM.e-9 & 2511110.00 & 5824528.00 & -898.95 & 898.95 & 2.414 \\
\hline VAM.x-1 & 2505664.99 & 5827112.95 & -1348.61 & 1348.61 & 2.410 \\
\hline VAM.x-2ST & 2510198.00 & 5828105.00 & -1069.98 & 1069.98 & 2.389 \\
\hline
\end{tabular}


Tabla 6.2. Rangos de valores de las distintas anomalías expresados en [mGal].

\begin{tabular}{|l|r|r|r|}
\hline \multicolumn{1}{|c|}{ Anomalía } & \multicolumn{1}{c|}{ Mínimo } & \multicolumn{1}{c|}{ Máximo } & \multicolumn{1}{c|}{ Media } \\
\hline Aire libre & 0.5 & 110.5 & 56.07 \\
\hline Bouguer simple & -110.1 & -38.23 & -70.23 \\
\hline Bouguer completa & -75.3 & -49.7 & -63.1 \\
\hline
\end{tabular}

\subsubsection{Espectro de potencia radial promediado}

Para hacer un análisis espectral hay que tener en cuenta que el campo de anomalías resulta en muchos casos de la superposición de los efectos producidos por fuentes situadas a distintas profundidades con longitudes de onda similares. En tales casos, el análisis espectral no es capaz de discriminar el efecto de cada una de las fuentes.

A efectos de evaluar las profundidades medias de los cuerpos fuentes de la anomalía gravimétrica dentro del edificio volcánico, se calculó el espectro de energía radialmente promediado de la grilla de la anomalía de Bouguer completa (Maus \& Dimri, 1996). Este espectro contiene información sobre la profundidad promedio de un conjunto de fuentes de una determinada frecuencia espacial, lo que además puede ser usado para separar el ruido existente en la señal a filtrar. La profundidad de los cuerpos se determina por medio del ajuste de rectas que aproximan tramos de la curva del logaritmo del espectro de potencia. $\mathrm{Si}$ el número de onda está expresado en unidades de ciclos por unidad de longitud, entonces la profundidad viene dada por $\bar{d}=\mathrm{m} / 4 \pi$ (sección 5.3.5.2, Capítulo V).

A partir del contenido espectral del campo de anomalías (figura 6.6), es posible seleccionar el rango de longitudes de onda o frecuencias de interés de las anomalías que se desean aislar. Esta información permite elegir en forma adecuada el número de onda de corte $k_{\mathrm{c}}$ o longitud de onda del filtro a utilizar. Se han distinguido dos grupos de profundidades bien definidas. Uno correspondiente a cuerpos más profundos, cuyo número de onda de corte es $0.16 \mathrm{~km}^{-1}$; y el otro a profundidades someras con un $k_{\mathrm{c}}$ de $0.41 \mathrm{~km}^{-1}$. A partir del valor de $k_{\mathrm{c}}$ de $0.65 \mathrm{~km}^{-1}$ se considera ruido en los datos. El primer tramo fue construido con 7 puntos y el segundo con 10. Estos dos tramos se han ajustado con una recta mediante mínimos cuadrados. El error del ajuste de la pendiente viene dado por la siguiente expresión (6.1):

$$
\varepsilon=\frac{m \sqrt{\frac{1-R^{2}}{n}}}{4 \pi}
$$

donde $\mathrm{m}$ es la pendiente de la recta, $R$ el coeficiente de correlación y $n$ el número de puntos en la recta. 
Reemplazando los valores obtenidos en la recta de ajuste para las fuentes más profundas, el error de ajuste resulta en 0.15 .

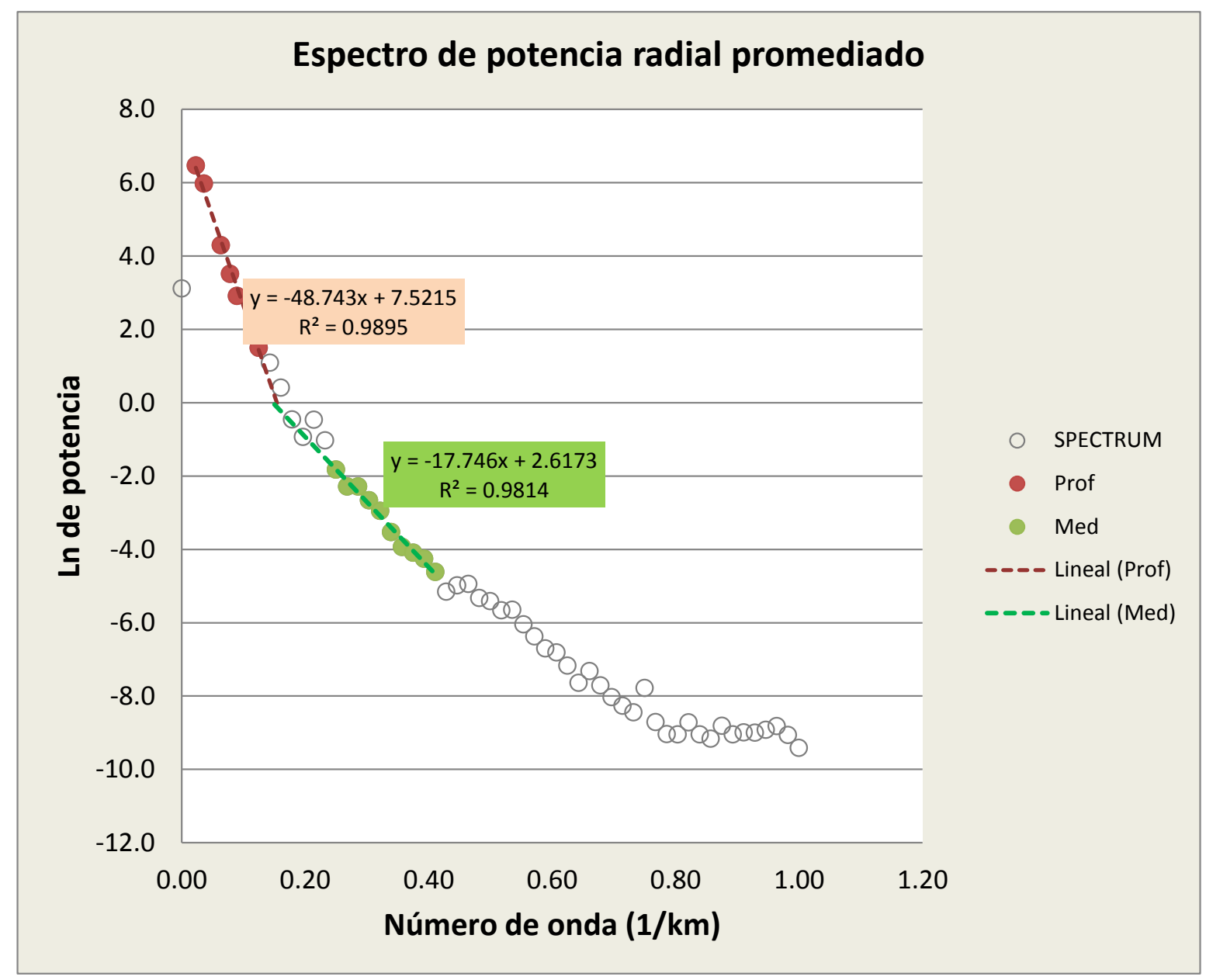

\begin{tabular}{|c|c|c|}
\hline Pendiente & Prof. Estimada $(\mathrm{km})$ & Rango nro de onda \\
\hline-48.743 & 3.879 & $0.023-0.16$ \\
\hline-17.746 & 1.412 & $0.19-0.41$ \\
\hline
\end{tabular}

Figura 6.6. Profundidad de las fuentes gravimétricas estimada a partir del espectro de energía logarítmico promediado radialmente del mapa de anomalías de Bouguer completa. El espectro corresponde a grupos de cuerpos con la misma profundidad media.

\subsubsection{Filtrado del campo de anomalías gravimétricas}

Como se mencionó en el Capítulo V, el tamaño de celda de la grilla del campo de anomalías y las dimensiones del área, determinan el mínimo y máximo números de onda $\left(k_{\min }, k_{\max }\right)$ de las anomalías registradas así como también la profundidad máxima de 
investigación. Por lo cual, las anomalías gravimétricas estarán caracterizadas por números de onda comprendidos entre $2.810^{-2} \mathrm{~km}^{-1}$ y $1 \mathrm{~km}^{-1}$. Utilizando el concepto de longitud de onda de Nyquist, todas las longitudes de onda por debajo de los 1000 m no podrán detectarse (sección 5.2, Capítulo V).

Un objetivo del filtrado de longitudes de onda es enfocar la atención en anomalías derivadas de un intervalo de profundidades específico. Si bien la profundidad es un factor determinante del espectro de una anomalía, se hace imposible alcanzar ese objetivo debido al ancho del espectro de anomalías y al efecto de la geometría y tamaño de fuentes sobre el mismo. Según Hinze (2013), las dimensiones del mapa con el que se está trabajando debería ser al menos seis veces la profundidad de la fuente de interés, o sea $\mathrm{Z}_{\mathrm{c}} \approx \lambda / 6$, donde $\lambda$ es la longitud de onda límite o crítica de una fuente cuya profundidad es $Z_{c}$. Esta regla supone que las fuentes residuales son masas concentradas, suposición que es válida sólo en algunas investigaciones. Se basa en el hecho de que la longitud de onda de la anomalía debida a una fuente concentrada y aislada es aproximadamente igual a $8 \mathrm{X}_{1 / 2}$, donde $\mathrm{X}_{1 / 2}$ es la distancia en una dada dirección que separa puntos donde la anomalía en cuestión tiene la mitad de su amplitud máxima. La profundidad máxima al centro de una masa concentrada es $\mathrm{Z}_{\mathrm{c}}=1.3 \mathrm{X}_{1 / 2}$. Por lo tanto $Z_{c}=1.3(\lambda / 8) \approx \lambda / 6$. En el caso de este estudio para localizar fuentes profundas a $30 \mathrm{~km}$ por ejemplo, se debería contar con un mapa de 180 x $180 \mathrm{~km}$ aproximadamente.

Al igual que en el caso magnético, la longitud de onda aparente de una anomalía gravimétrica, viene dada por su extensión longitudinal sobre el mapa la cual está relacionada con la profundidad del cuerpo que la genera. De este modo, cuerpos profundos darán como respuesta anomalías de longitudes de onda grandes con gradientes suaves, mientras que cuerpos someros y pequeños producen anomalías de onda corta.

El campo de anomalías de Bouguer completa (figura 6.5) contiene información proveniente de estructuras geológicas profundas (campo regional), generalmente el basamento; y someras (campo residual o local) debido a estratos geológicos con mayor o menor densidad que las rocas circundantes. La separación de estos efectos es indispensable para la interpretación de los mismos, ya que la componente regional podría enmascarar las respuestas producidas por las fuentes superficiales. Si bien la definición de componente regional y residual nunca podrá ser totalmente objetiva, la separación de ambos efectos dependerá del objetivo de la interpretación, de la experiencia del intérprete, de la calidad de los datos y del conocimiento de la geología. El proceso de separación de campos puede llevarse a cabo mediante métodos gráficos y analíticos. Los segundos son los más utilizados, y entre ellos podemos mencionar el de regresión y ajuste de superficies polinómicas, conocidas también como superficie de tendencia; prolongación o continuación analítica de campos, filtrado por medio de la transformada de Fourier, entre otros. El procedimiento de separación de señales se realiza en forma simple en el dominio de la frecuencia mediante la transformada de Fourier 2D sobre la grilla de anomalía. El teorema de Parseval garantiza que la información contenida en el dominio espacial y en el dominio de la frecuencia, son iguales por lo que no hay pérdida de información al cambiar de dominio (Blakely 1995).

El método de superficie de tendencia, supone que la componente regional de $\mathrm{ABC}$ se 
puede representar por una superficie suave que corresponde a un polinomio de bajo grado en las coordenadas de posición horizontal $x$ e y. Esta superficie será la que mejor se ajuste a los puntos de datos distribuidos en el espacio, usando el método de mínimos cuadrados, es decir que hace mínima la suma de cuadrados de la diferencia entre la anomalía y la superficie polinómica. La anomalía residual se evalúa punto a punto, sustrayendo a la $\mathrm{ABC}$ la componente regional. Cuanto más alto el grado del polinomio, mejor ajustará los puntos observados pero la componente regional puede carecer de sentido geológico. El grado del polinomio es un parámetro crítico en este método y es importante el juicio del intérprete para la selección del mismo, el cual debería ser el menor posible que represente la mayor parte de la tendencia regional. La curva ajustada debe pasar por el valor medio de los valores de gravedad, de modo que las anomalías residuales se distribuyan igualmente entre valores positivos y negativos (Lowrie, 2007).

Para la obtención de la anomalía residual, se sustrajeron dos superficies de tendencia regional, de grados 1 y 3 (figura 6.7) sobre todos los puntos de la anomalía de Bouguer completa. Sobre el perfil orientado SO-NE que pasa por el mínimo gravimétrico (figura 6.8) se graficaron la $\mathrm{ABC}$ y sus componentes regionales y residuales (figuras 6.9 y 6.10 ) obtenidas a partir de las superficies de tendencia de la figura 6.7.

La transformación de un plano a otro o continuación analítica fue discutida en la sección 5.3.2 del Capítulo V. El proceso de filtrado para realizar la continuación ascendente incluye la preparación de la grilla original en el dominio espacial, es decir, se expande la grilla hasta un cuadrado con dimensiones aceptables para la FFT. Una vez aplicado el filtro en el dominio de las frecuencias, se llevan los datos procesados al tamaño y forma original de la grilla.

La prolongación hacia arriba se realizó en el mapa de anomalías de Bouguer completa considerando varias alturas. La figura 6.10 a y c muestra el resultado de la continuación ascendente a 10 y $20 \mathrm{~km}$, respectivamente. La componente residual de la anomalía se obtuvo calculando la diferencia entre la grilla de anomalía de Bouguer completa y la anomalía regional obtenida de la prolongación. En la figura 6.12 se representan la $A B C$ y las componentes regionales y residuales obtenidas de la continuación ascendente a lo largo del perfil de la figura 6.8 .

Otro de los métodos utilizados para realizar la separación de componentes es mediante filtrado por número de onda y uno de los más usados en métodos potenciales es el de Butterworth, dado por la ecuación (6.2):

$$
L(k)=\frac{1}{\left[1+\left(\frac{k}{k_{0}}\right)^{n}\right]}
$$

donde $k_{0}$ es el número de onda central del filtro y $n$ el grado de la función filtro.

El número de onda de corte usado es de $0.09 \mathrm{~km}^{-1}$ (longitud de onda de $11 \mathrm{~km}$ ) el cual 
se eligió a partir de los resultados obtenidos del espectro radial promediado de la figura 6.6 y $\mathrm{n}=8$ el grado de la función del filtro aplicado. De esta forma se obtuvo la componente regional de la anomalía completa de Bouguer (figura 6.13).
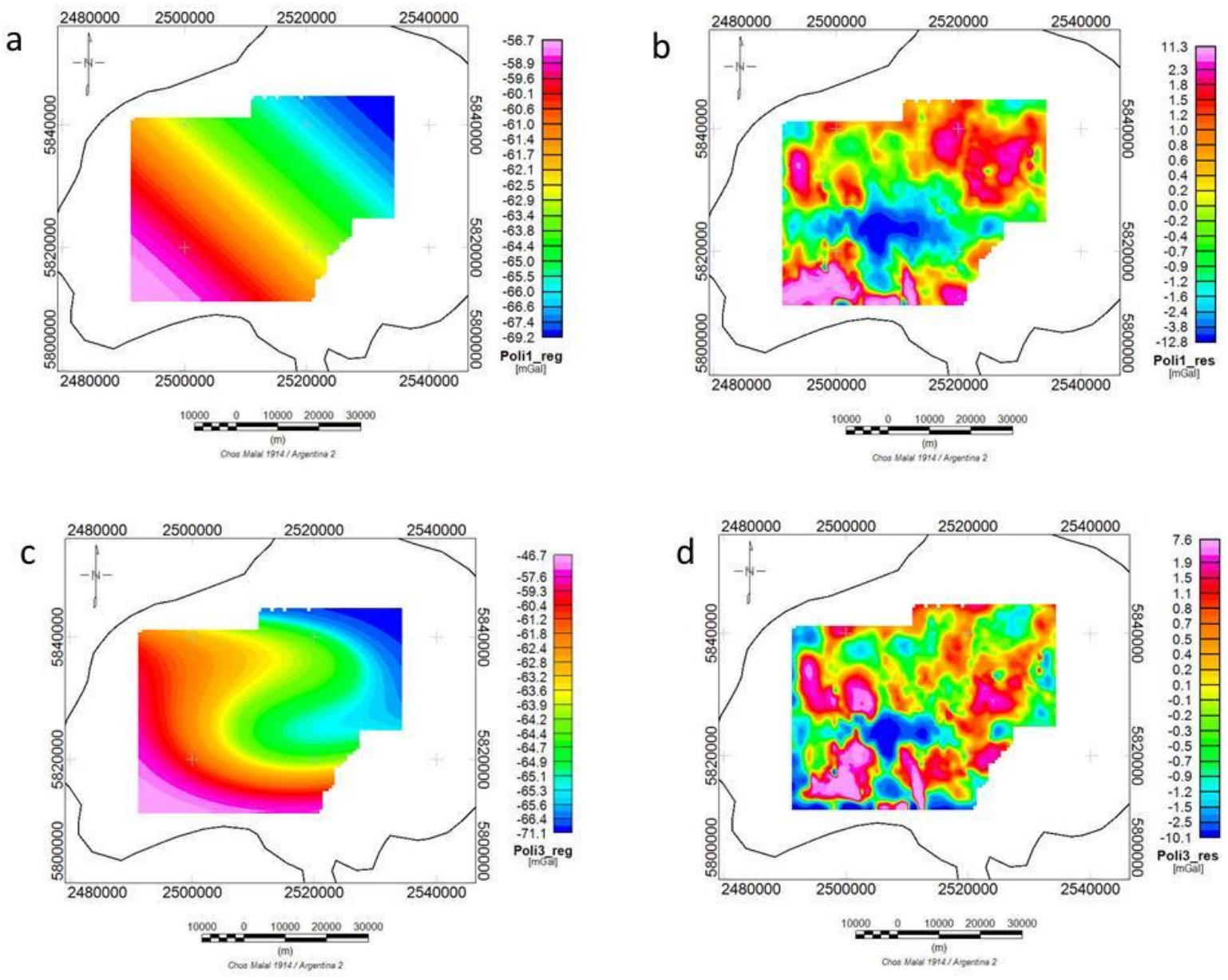

Figura 6.7. a y c) Anomalía regional con ajuste polinómico de grado 1 y 3 , respectivamente. b y d) Anomalía residual resultado de la remoción de la anomalía regional en a) y c) respectivamente, a la anomalía de Bouguer completa. 


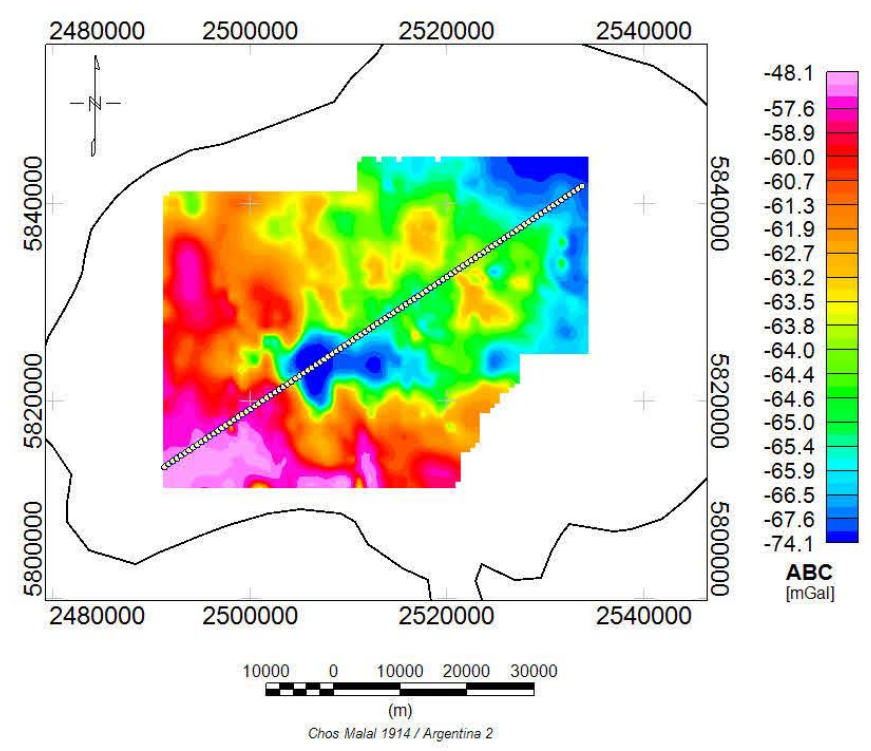

Figura 6.8. Perfil SO-NE sobre el cual se grafica la respuesta de la $A B C$ y sus componentes regionales y residuales (figuras $6.9,6.10$ y 6.12 ) obtenidas con los métodos mecionados en el texto.

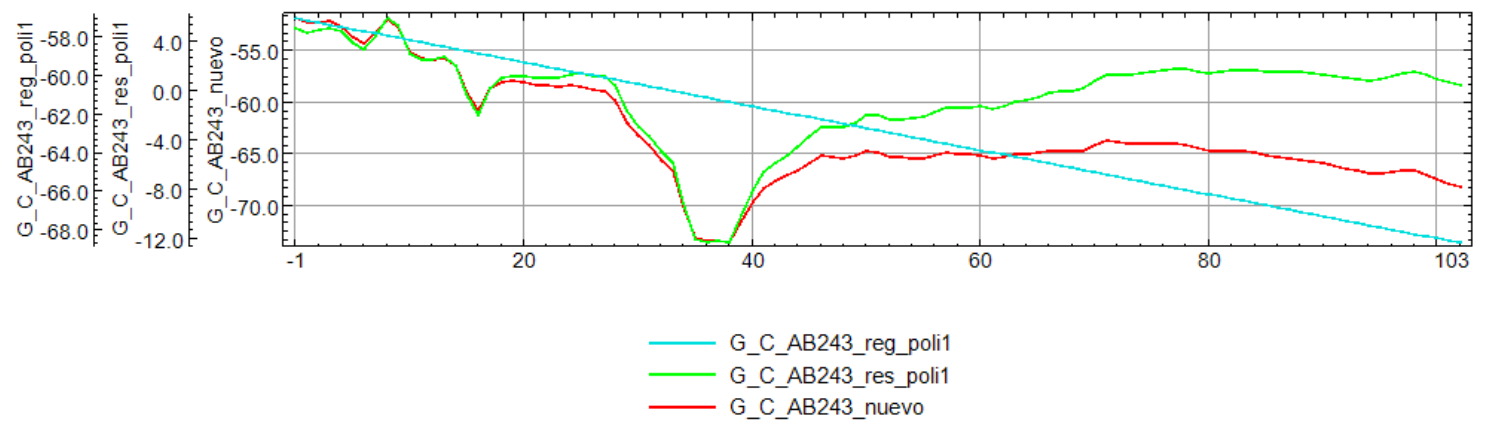

Figura 6.9. Anonalía de Bouguer ABC, junto a las componentes residual y regional resultado de aplicar una remoción de tendencia lineal sobre el perfil indicado en el mapa. Curva roja: ABC, verde: componente residual y celeste: componente regional.

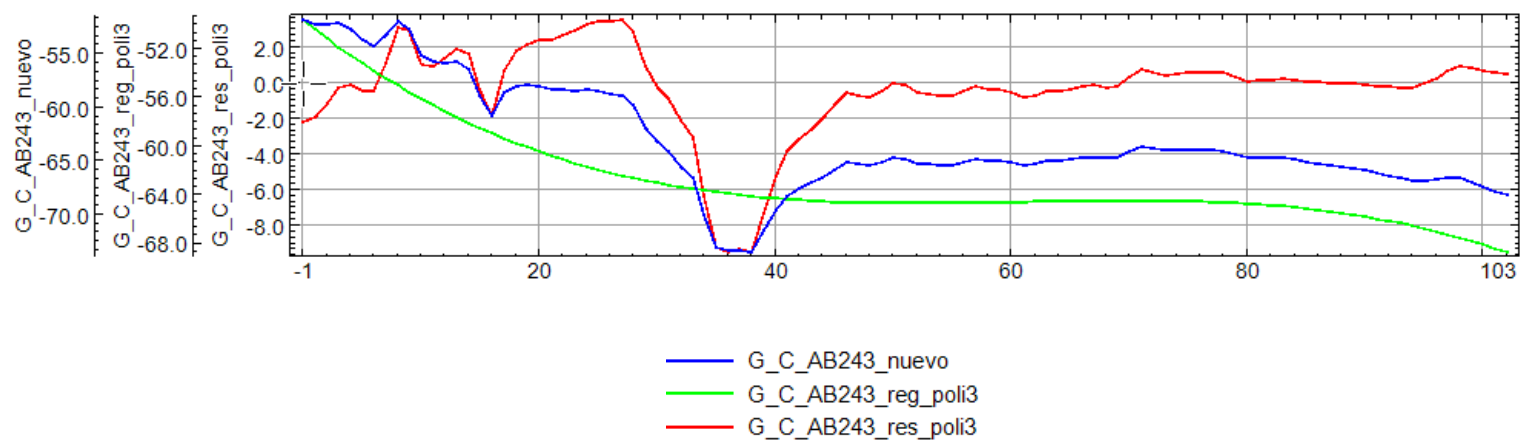

Figura 6.10. Anonalía de Bouguer ABC, junto a las componentes residual y regional resultado de aplicar una remoción de tendencia de polinomio de grado 3 sobre el perfil indicado en el mapa. Curva azul: $A B C$, roja: componente residual y verde: componente regional. 
a

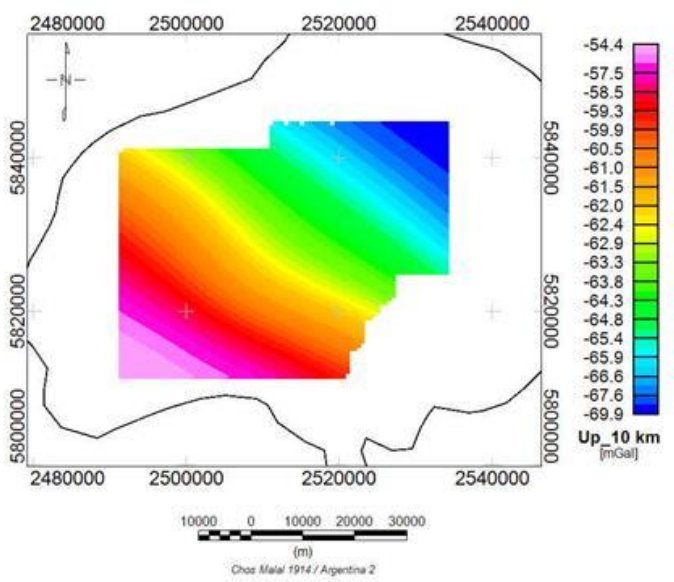

C

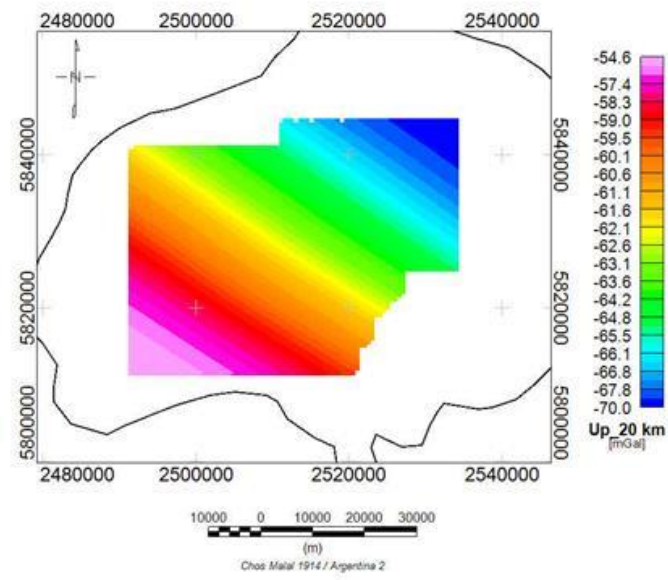

b
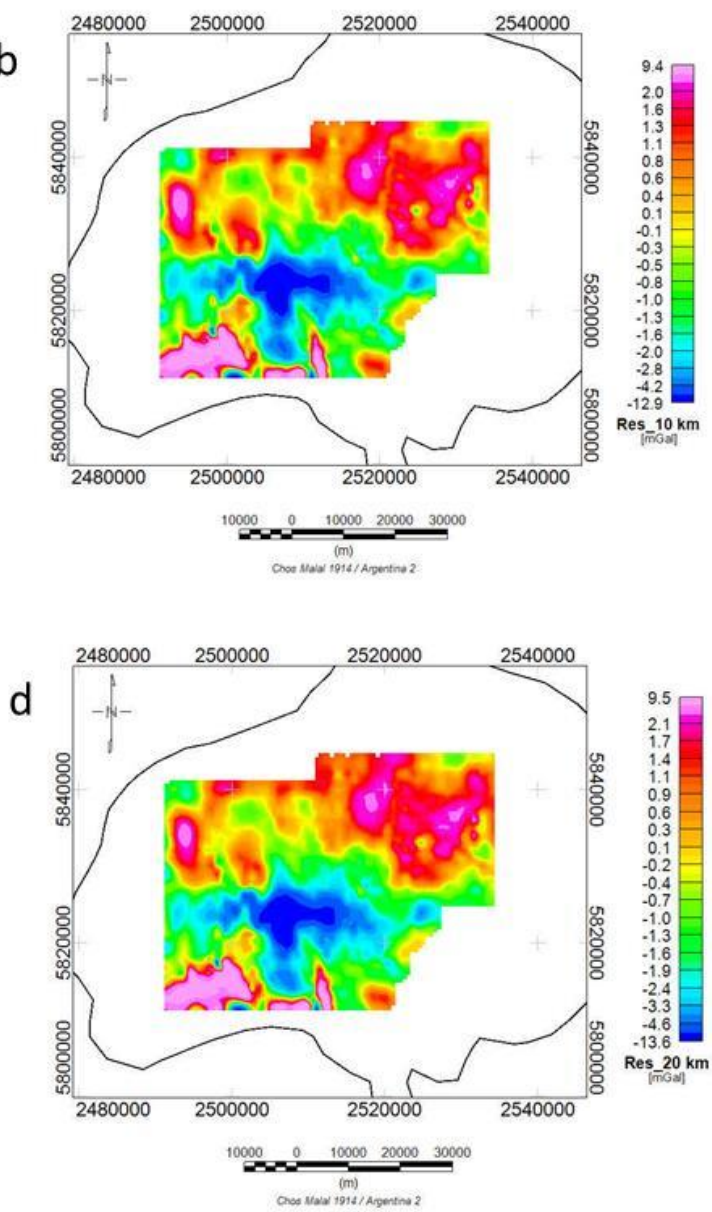

Figura 6.11. a y c) Componente regional resultado de la prolongación ascendente de la anomalía de Bouguer completa a 10 y $20 \mathrm{~km}$, respectivamente. b y d) Anomalía residual de Bouguer resultado de la diferencia entre la anomalía de Bouguer completa y la componente regional a 10 y 20 km, respectivamente.
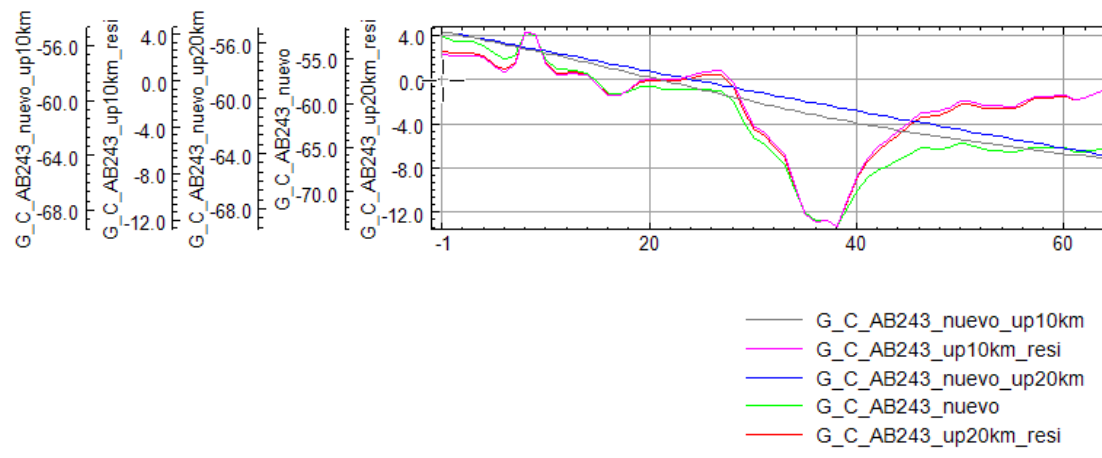

Figura 6.12. Anonalía de Bouguer ABC, junto a las componentes residual y regional resultado de la continuación ascendente a 10 y $20 \mathrm{~km}$. Curva verde: $A B C$, gris y azul: continuación ascendente a 10 y $20 \mathrm{~km}$, respectivamente, rosa y roja: componente residual resultado de la continuación a 10 y $20 \mathrm{~km}$, respectivamente. 

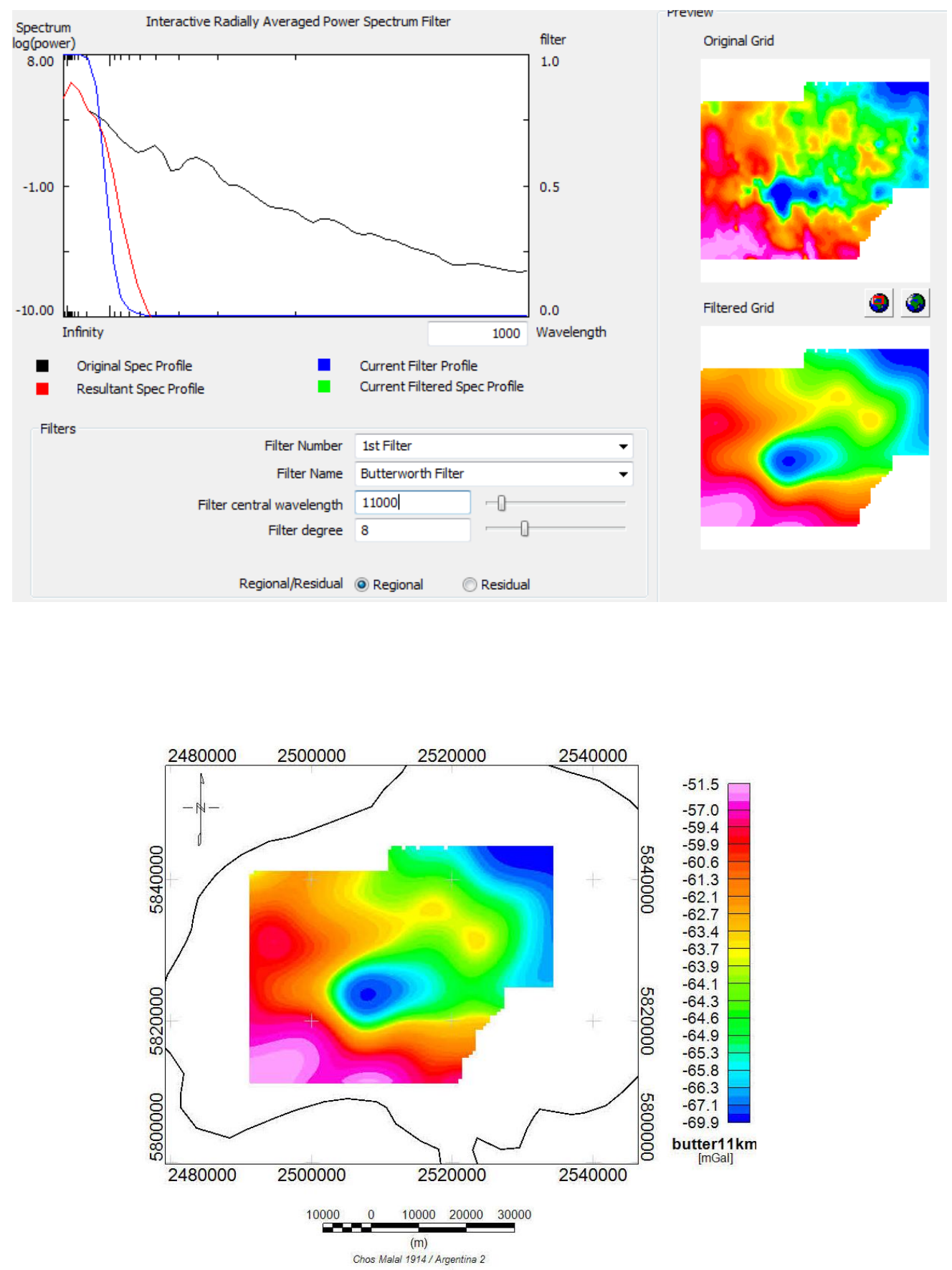

Figura 6.13. Filtro de Butterworth aplicado a los datos de anomalía de Bouguer completa. Se usó un número de onda de corte de $0.09 \mathrm{~km}^{-1}$, o sea una longitud de onda de $11 \mathrm{~km}$ para obtener la componente regional. 
En todos los métodos de separación utilizados, la amplitud de la componente regional está comprendida entre -70 y -55 mgales y la componente residual entre -13 y 9 mGal aproximadamente. La componente regional obtenida con un polinomio de primer orden (figura 6.7 a) y el resultado de la continuación ascendente (figuras 6.11 a y c) muestra que el patrón general es el mismo que el de la anomalía de Bouguer completa, cuya tendencia es SONE. Cualquiera sea el método utilizado en la separación, la componente residual siempre presenta el mismo comportamiento. Esto confirma que la componente principal de la respuesta medida proviene de los primeros kilómetros del paquete sedimentario y el residuo se debe a las frecuencias más altas de los cuerpos de escala más pequeña.

\subsection{Datos gravimétricos terrestres regionales}

Los datos gravimétricos terrestres cubren gran parte de la cuenca Neuquina. Estos levantamientos fueron adquiridos por YPF S. A. con objetivos exploratorios petroleros, en los que se midieron alrededor de 31.000 estaciones.

Todas las grillas se realizaron con el método de mínima curvatura con un espaciamiento de $2500 \mathrm{~m}$. En el cráter central del volcán no hay cobertura de datos, por lo cual la grilla en esa parte genera una interpolación incorrecta, como el círculo azul en la figura 6.14.

En el sector este y sur del mapa de gravedad, la distribución de los datos tiene un diseño regular (figura 6.14). Estos puntos parecieran ser el producto de la digitalización de mapas en lugar de la posición real de los datos medidos. Además, por la época de registración (antes de la década del 80) no pueden ser datos provenientes de relevamientos aéreos. Este hecho motiva a utilizar estos datos de forma descriptiva para enmarcar el volcán Auca Mahuida en un contexto regional.

La figura 6.15 representa la anomalía de aire libre. Tres máximos importantes aparecen en dirección NO-SE coincidentes con altos de topografía.

La anomalía de Bouguer se calculó con la densidad característica típica del relleno sedimentario en la cuenca $\left(2.67 \mathrm{~g} / \mathrm{cm}^{3}\right)$. No se tomó la de $2.43 \mathrm{~g} / \mathrm{cm}^{3}$ porque representa sólo una parte pequeña de todos los datos regionales. En este caso como se cuenta con los valores de gravedad observada (figura 6.14) la anomalía de Bouguer simple y con corrección topográfica (figuras 6.16 y 6.17, respectivamente) se obtuvieron a partir de estos datos. La grilla de $\mathrm{ABC}$ tiene una extensión menor debido al tamaño del modelo digital del terreno utilizado. 


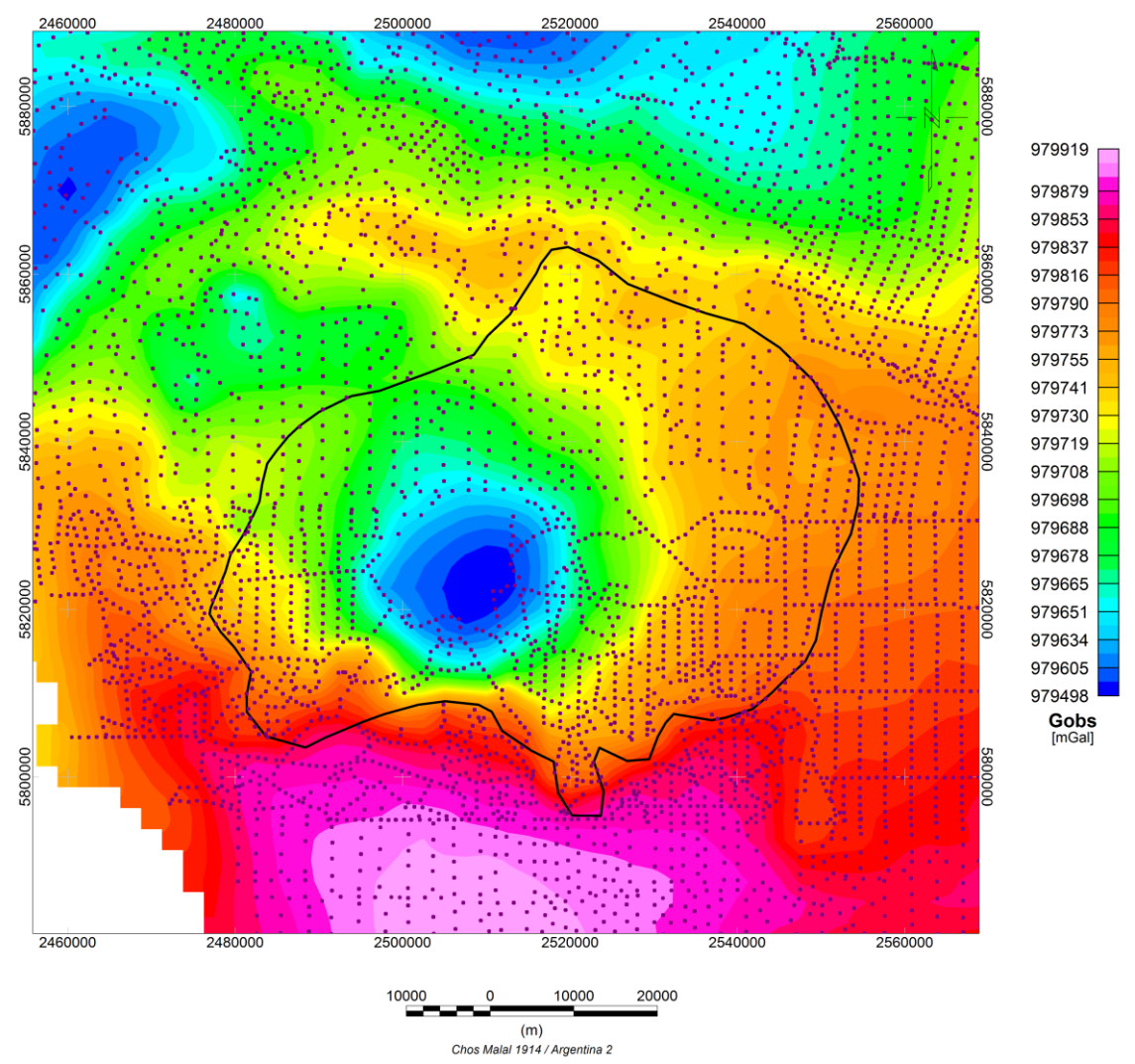

Figura 6.14. Gravedad observada. Los puntos en color morado corresponden a los datos de gravedad terrestres medidos en la cuenca Neuquina.

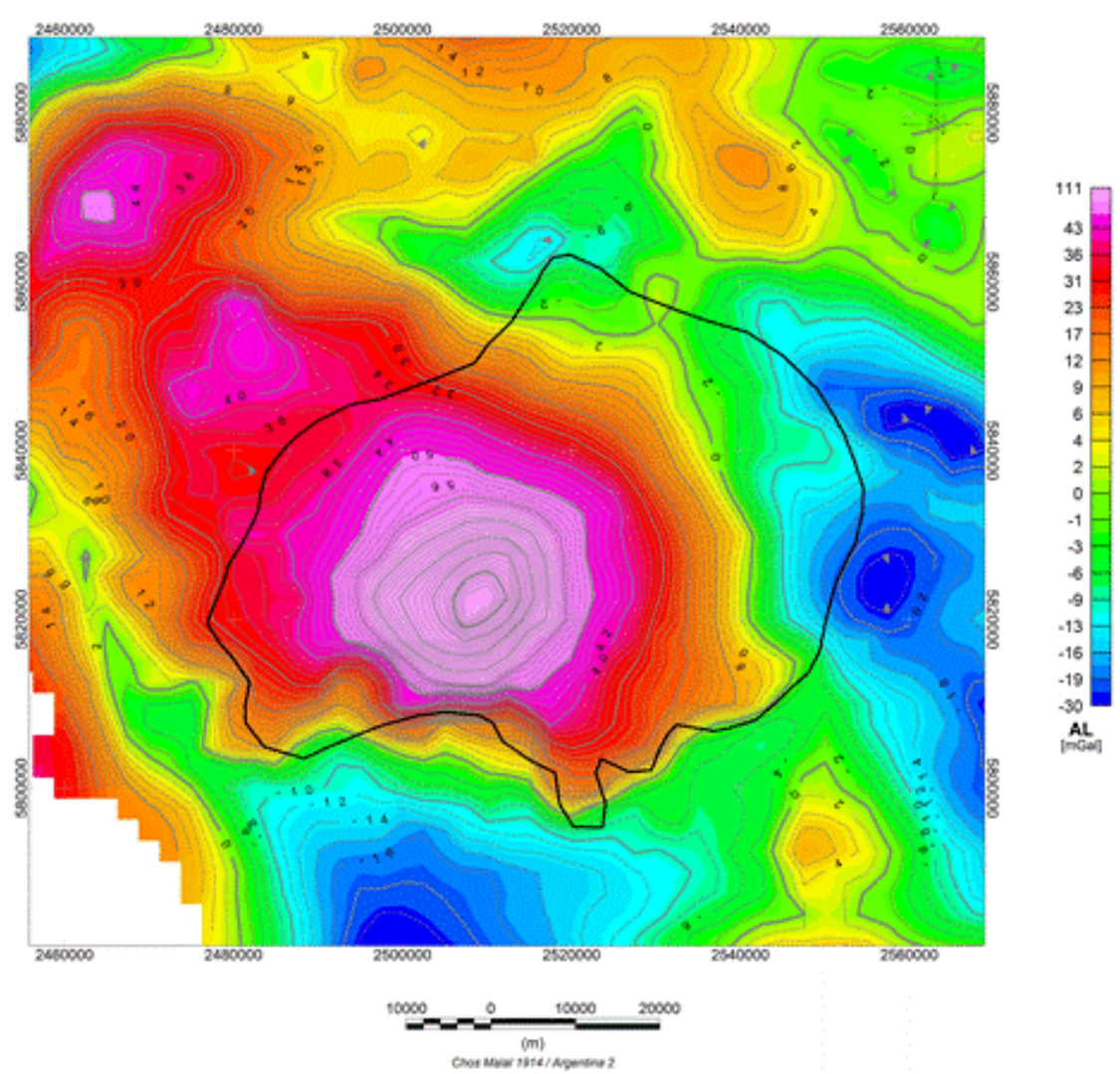

Figura 6.15. Anomalía de aire libre de los datos regionales terrestres. 


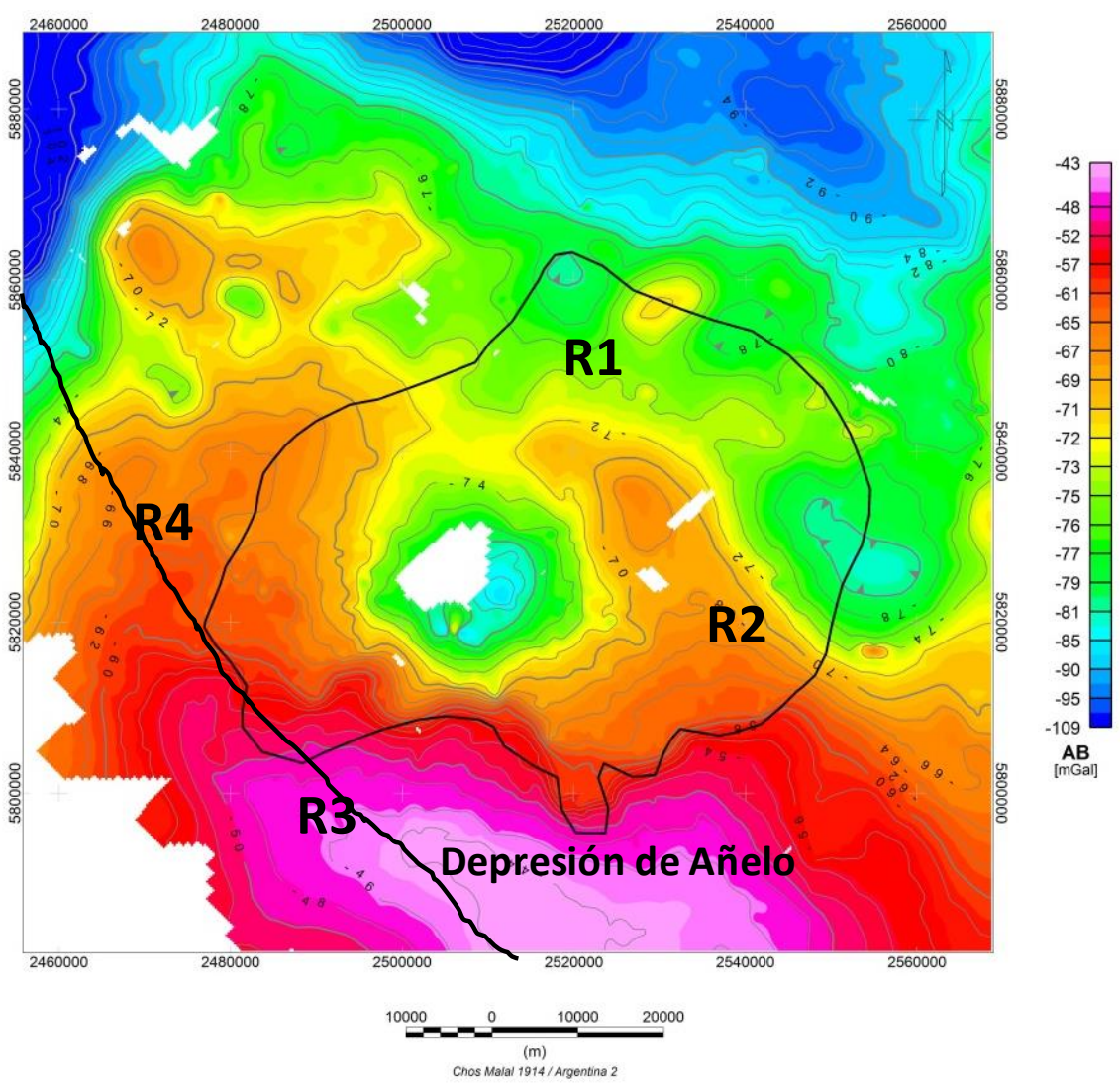

Figura 6.16. Anomalía de Bouguer simple de los datos regionales terrestres obtenida con una densidad de reducción de $2.67 \mathrm{~g} / \mathrm{cm}^{3}$. El VAM está ubicado entre un mínimo (norte) y máximo (sur) relativo. $\mathrm{R} 1, \mathrm{R} 2$, $\mathrm{R} 3$ y $\mathrm{R} 4$ representan las anomalías magnéticas identificadas en la figura 5.11.

Los rangos de valores de la anomalía de Bouguer de ambos relevamientos en la zona de estudio, aerogravimetría del VAM y regional de la cuenca Neuquina son consistentes. El mínimo relativo en la zona central del volcán está presente en la anomalía de Bouguer de ambos relevamientos, con un rango que varía entre -75 y - $65 \mathrm{mGal}$, aproximadamente.

Se superpusieron los nombres de las anomalías magnéticas identificadas como R1, R2, R3 y R4 sobre el mapa de la figura 6.16. En este mapa se advierte un máximo relativo elongado en dirección NO-SE en correspondencia con el Bajo o Depresión de Añelo, caracterizado por una anomalía magnética negativa y positiva (R4 y R3 respectivamente en figura 5.11). El Bajo de Añelo es un rasgo estructural regional de orientación NO-SE que divide al engolfamiento Neuquino en dos dominios: al NE la plataforma nororiental, y hacia el sur y oeste, la plataforma sur y la dorsal de Huincul. Se extiende a lo largo de unos $280 \mathrm{~km}$ desde General Roca (Provincia de Río Negro) hasta el noroeste del volcán Auca Mahuida (Pángaro, et al., 2011). Datos de perforaciones e información de sísmica 2D y 3D, revelan que esta estructura cuya expresión gravimétrica es una anomalía de Bouguer positiva (máximo relativo) corresponde a un depocentro sedimentario de gran espesor. Los pozos más profundos que llegan a depósitos del Grupo Choiyoi, alcanzan los 5300 mbbp (metros bajo boca de pozo). Esto es consistente con la hipótesis planteada por Sigismondi (2011) respecto 
a que la anomalía de Bouguer (máximo relativo) sobre el eje del depocentro sedimentario es el resultado de atenuamiento cortical por mecanismo de cizalla simple, lo cual eleva el límite del manto astenosférico, que produce la anomalía gravimétrica positiva regional.

Hacia el norte (R1), los valores de gravedad diminuyen, alcanzando los -90 mGal, mientras que en el mapa magnético esta región está representada por un máximo. R2 se presenta como un espolón gravimétrico positivo con una disminución hacia el este, en cambio en el campo de las anomalías magnéticas el máximo es mayor arealmente y más pronunciado.

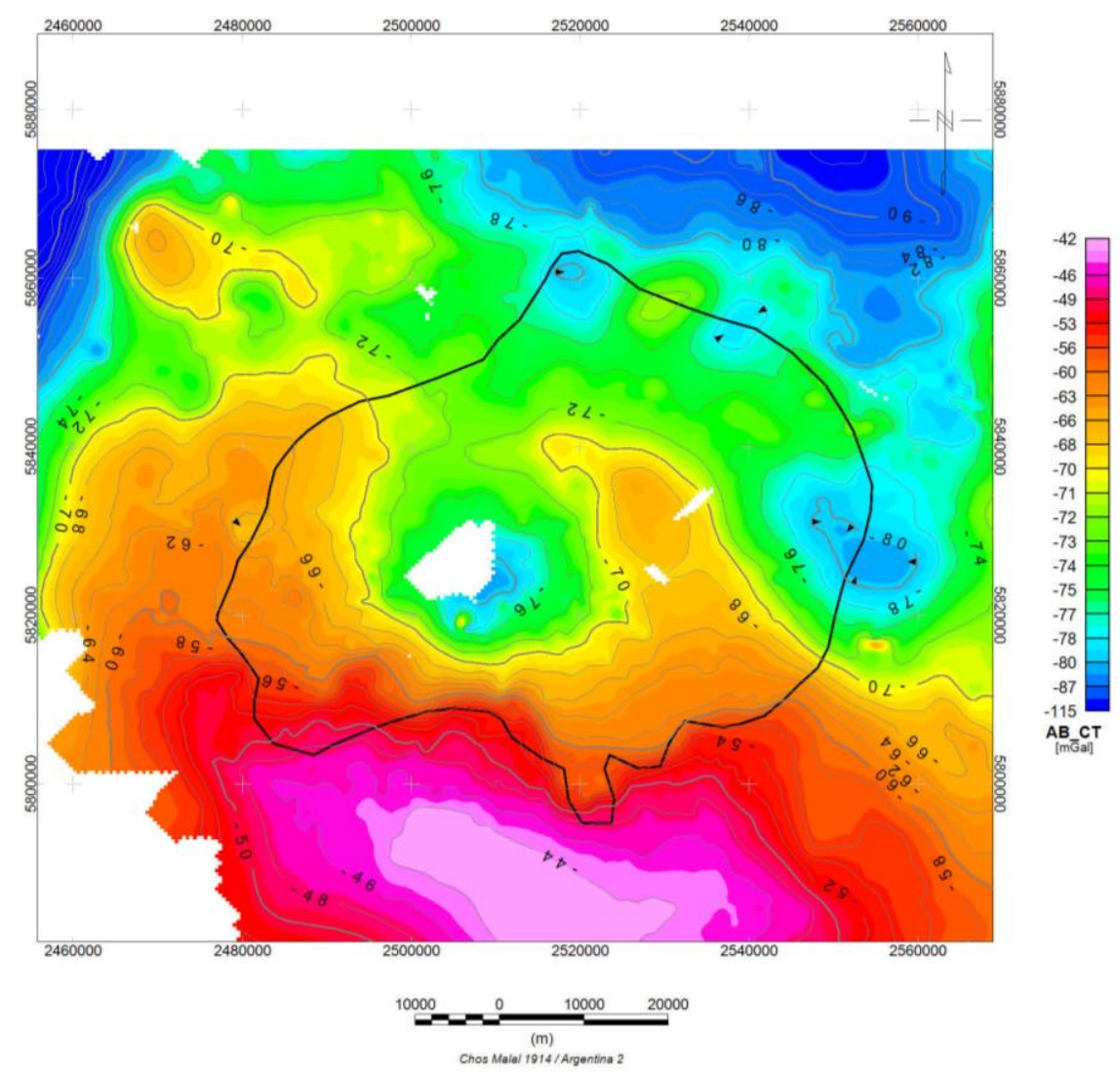

Figura 6.17. Anomalía de Bouguer completa de los datos regionales terrestres obtenida con una densidad de reducción de $2.67 \mathrm{~g} / \mathrm{cm}^{3}$. La corrección topográfica se realizó con el modelo digital del terreno de la figura 3.1.

Como se verá en la sección siguiente, el dato sísmico juega un rol importante en las zonas aledañas al cráter central del volcán, especialmente donde la relación señal-ruido mejora y donde no hay cobertura de datos aerogravimagnéticos de alta resolución del VAM.

\subsection{Conceptos básicos de sísmica de reflexión}

La exploración sísmica incluye la adquisición, procesamiento y análisis de los eventos generados por un microsismo expresamente provocado. El método sísmico de reflexión 
consiste en registrar los tiempos de llegada de las ondas reflejadas en los contactos del subsuelo a una serie de detectores superficiales denominados geofónos o hidrófonos ya sea se registre en tierra o mar, respectivamente. Para la generación de ondas sísmicas se emplean diferentes fuentes, entre las que se encuentran, entre otras, los cañones de aire (registración marina), explosivos y camiones vibradores (registración terrestre).

Se denomina impedancia acústica a la resistencia que ejerce un medio homogéneo al paso de una onda de sonido. La misma se define como:

$$
Z=\rho . v
$$

donde $\rho$ es la densidad y $v$ la velocidad de propagación de la onda.

Se puede definir al dato sísmico como aquel que resulta de registrar en escala de tiempo, una onda compleja que, generada por un microsismo provocado, ha viajado a través de la Tierra transmitiéndose y reflejándose en los diferentes contrastes de impedancia acústica del subsuelo. Tales contrastes están dados por cambios en las propiedades físicas de las rocas debido a variaciones en la estratigrafía. Su magnitud se mide en términos de coeficientes de reflexión los cuales contienen información muy útil sobre las características del subsuelo.

Cuando una onda sísmica incide normalmente sobre la interfaz plana entre dos medios elásticos $i$, e $i+1$, el coeficiente de reflexión $(R)$ asociado resulta:

$$
R_{i+1}=\left(Z_{i+1}-Z_{i}\right) /\left(Z_{i+1}+Z_{i}\right)
$$

siendo $Z_{i}$ y $Z_{i+1}$ las impedancias acústicas de los respectivos medios.

Las reflexiones se producen cuando hay un contraste importante de impedancia acústica entre las capas debido a las variaciones de las propiedades elásticas del medio, tanto de la densidad, velocidad o una combinación de ambas y cuando las longitudes de onda son inferiores al tamaño característico de dichas capas.

\section{Modelo de traza sísmica}

La propagación de una onda sísmica a través de un medio, origina el desplazamiento de las partículas que lo componen. Existen dos tipos básicos de ondas elásticas de acuerdo con la dirección relativa del movimiento de las partículas respecto de la dirección de propagación de la onda: las ondas compresionales (ondas P) en las cuales la dirección del movimiento de las partículas es paralela a la dirección de propagación y las ondas transversales (ondas S) en las que la dirección del movimiento es perpendicular a la dirección de propagación de la onda.

A los efectos de simplificar el análisis de la información geológica contenida en el dato sísmico, se asumirá que éste resulta solamente de ondas P planas incidiendo y reflejándose 
verticalmente en interfaces horizontales y que la operación matemática "convolución" es capaz de sintetizar el "viaje" a través de la Tierra de una ondícula que permanece invariante con el tiempo.

La hipótesis de planaridad implica que las interfaces reflectoras están suficientemente lejos de la fuente para considerar el frente de onda esférico aproximadamente plano.

El resultado de la registración, conocido como traza sísmica, $T(t)$, puede sintetizarse mediante la convolución de la serie de los coeficientes de reflexión, $R(t)$, con una ondícula $O(t)$, como se indica en (6.12):

$$
T(t)=R(t) * O(t)
$$

donde $t$ es el tiempo empleado por la onda sísmica para recorrer el camino desde la fuente hasta las interfaces reflectoras y desde allí hasta el receptor. Una ondícula sísmica es una forma de onda de corta longitud temporal resultante de la fuente de excitación, la respuesta del instrumento de registración y la absorción del medio. Podemos mencionar entre los varios tipos de ondículas, la Ricker, Klauder, Ormsby y Butterworth (Longo, 2000) con diferentes características. La ondícula Ricker es generada tanto en el dominio del tiempo como en el de las frecuencias y los valores de fase varían entre $0^{\circ}$ y $360^{\circ}$. La ondícula Klauder se genera en el dominio del tiempo y se transforma al dominio de la frecuencia para elegir la fase. La ondícula Ormsby posee la forma de un filtro trapezoidal. La ondícula Butterworth se genera en el dominio de las frecuencias y los valores de fase pueden variar entre $0^{\circ}$ y $360^{\circ}$. Debe mencionarse que, aún manteniendo el espectro de amplitud invariante, la forma de la ondícula puede cambiar modificando su fase (Yilmaz, 1988).

Se denomina tiempo doble al tiempo que transcurre en el viaje de la ondícula hacia un punto de reflexión y su regreso desde la fuente al receptor a un plano de referencia sísmica común (Schlumberger, 1987).

\subsection{Interpretación del dato sísmico}

Como se comentó en el Capítulo III, los datos sísmicos 3D no cubren la zona central del cráter y las líneas sísmicas en esta zona son de muy baja relación señal-ruido debido al gran espesor de rocas basálticas de superficie (figura 6.18). De todos modos, la interpretación de las principales formaciones de interés así como los filones intruídos en la secuencia sedimentaria, contribuyen a encontrar una tendencia y caracterizar los cuerpos ígneos.

A los efectos de realizar la interpretación sísmica de los principales horizontes es necesario obtener leyes de velocidad para realizar la conversión de tiempo doble del dato sísmico a profundidad del subsuelo. Las leyes de velocidad se obtienen a partir de la calibración entre la sísmica y los pozos utilizando perfiles sónicos y de densidad, con los cuales se obtiene un sismograma sintético a los efectos de comparar con el dato sísmico. 
En el área de estudio, existen perforaciones con perfiles sónicos y densidad que nos permitirán realizar la calibración pertinente.

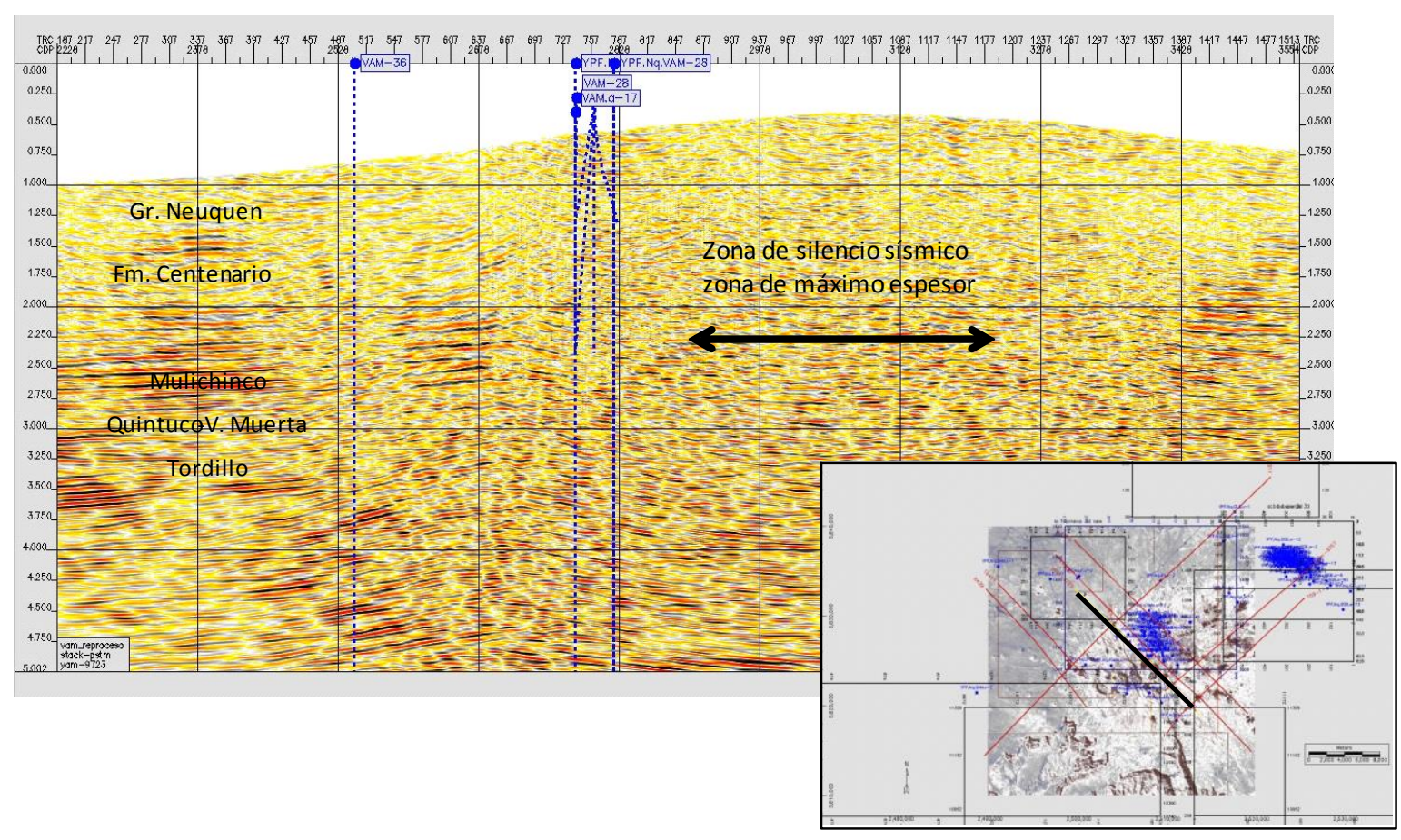

Figura 6.18. Línea sísmica 2D yam-9723 NO-SE reprocesada en 2012 representada en color negro.

\subsubsection{Calibración de sísmica con perfiles de pozos}

Para determinar los horizontes a interpretar durante la interpretación sísmica es necesario volcar la información obtenida de la perforación de los pozos sobre la sección sísmica. Los sismogramas sintéticos constituyen una herramienta esencial para lograr esta integración a través de trazas sintéticas generadas a partir de los perfiles sónicos y de densidad mediante la convolución de una ondícula con la serie de coeficientes de reflexión sísmicos del modelo, lo que permite realizar la calibración entre las secciones sísmicas y los perfiles de pozo.

También se realiza sísmica en el pozo para obtener las velocidades directamente, a esta registración se la denomina VSP (Vertical Seismic Profile).

Se realizó la calibración sísmica en el pozo utilizando los datos disponibles en la zona de estudio y los pases formacionales provistos por los geólogos de YPF S. A. La correlación entre los sismogramas sintéticos y los datos sísmicos en general es de buena calidad, disminuyendo hacia la zona de mayor topografía del volcán (zona central). Para los pozos que carecen de registros de densidad se asumió una densidad constante de $2.4 \mathrm{~g} / \mathrm{cm}^{3}$, que es un valor promedio representativo de la columna sedimentaria en la zona. El impacto de la densidad en el resultado de la serie de coeficientes de reflexión y en consecuencia en los 
sismogramas sintéticos, salvo en situaciones litológicas excepcionales, es mínima debido a la diferencia de rangos de densidad en comparación con los de velocidad.

Para la elaboración de los sismogramas sintéticos se utilizó el programa Synthetics de Geoframe $^{\mathrm{TM}}$. La figura 6.19 muestra la calibración del pozo VAM.x-1.

Un buen ajuste entre el sismograma sintético y la sección sísmica posibilita, con cierto grado de confianza, la transferencia de la información del pozo proveniente de perfiles (pases de formación, litología, etc.) a la sección sísmica correspondiente. Para lograr este ajuste, además de contar con los perfiles sónicos y de densidad con su correcto control de calidad, es imprescindible efectuar una cuidadosa elección de la ondícula de acuerdo con los parámetros de registración y procesamiento utilizados para obtener la sección sísmica (Schlumberger, 1987), además de incluir la información de checkshots o VSP (Vertical Seismic Profile), de estar disponible.

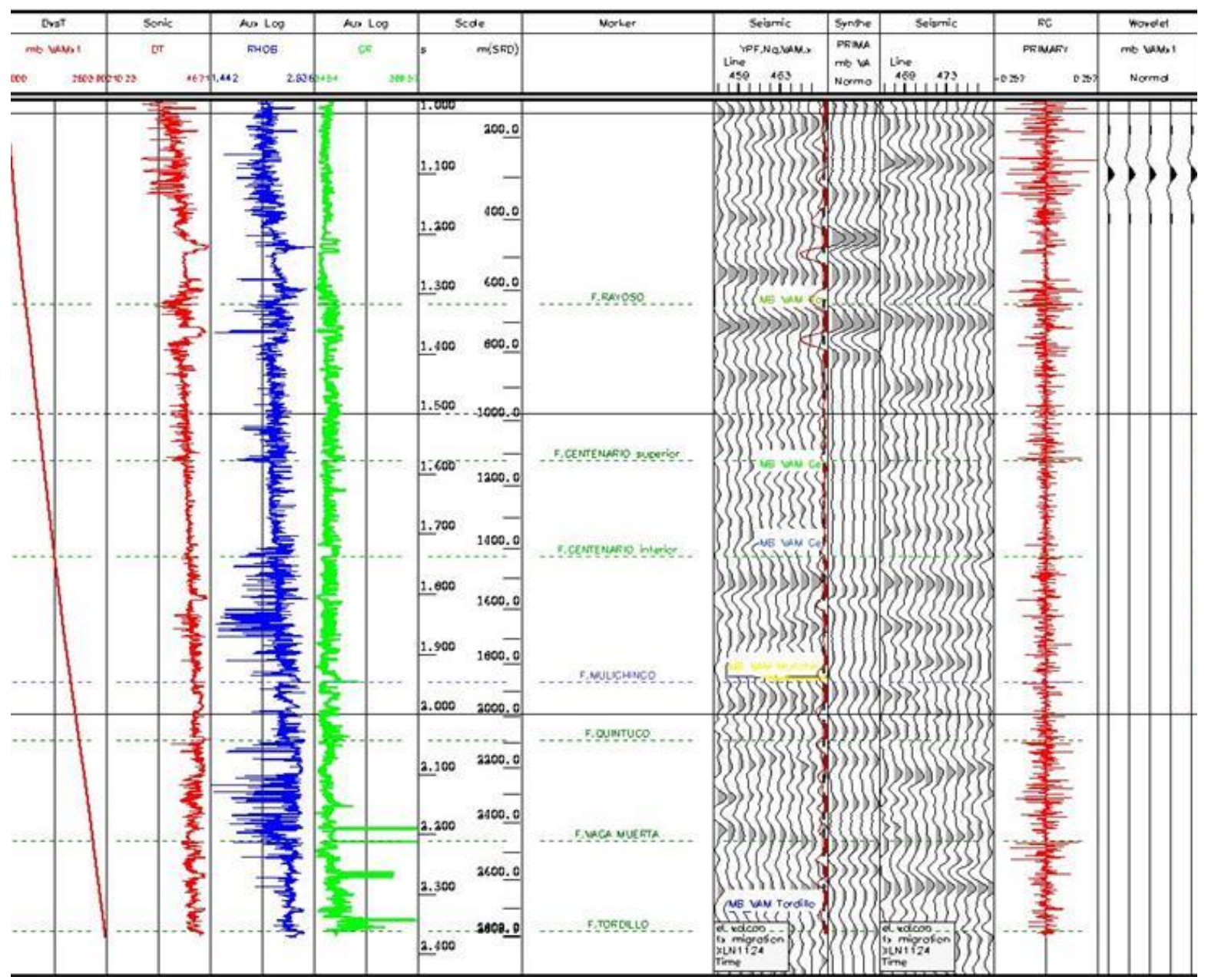

Figura 6.19. Sismograma sintético del pozo VAM-x.1. De izquierda a derecha: ley de velocidad obtenida del sismograma, perfil sónico (rojo), densidad (azul), rayos Gamma (verde); escala de tiempo y profundidad, pases formacionales, porción de la sección sísmica alrededor del pozo a izquierda y derecha y en el centro el sismograma sintético. Serie de coeficientes de reflexión en color rojo y ondícula. 


\section{Registros sónicos (DT)}

El registro sónico es un registro del tiempo que requiere una onda acústica para transmitirse en un espesor determinado de formación, el cual se denomina tiempo de tránsito $\Delta \mathrm{t}$. El cociente entre la distancia recorrida y el tiempo de tránsito es la velocidad de la propagación de la onda sísmica en dicho espesor. El tiempo de tránsito para una formación determinada depende de su litología, porosidad y de muchos otros factores (Schlumberger, 1995). Cuando se conoce la litología, el registro sónico es muy útil como registro de porosidad, comúnmente calculada mediante la ecuación de Wyllie (Sheriff, 1991). A un aumento de porosidad corresponde una disminución de la velocidad del sonido en la roca y por lo tanto aumenta el tiempo de tránsito (Schlumberger, 1995). La frecuencia con que trabaja la herramienta del perfil sónico está en el rango de $25000 \mathrm{~Hz}$.

En términos generales las velocidades sónicas en litologías de las formaciones presentes en el subsuelo fluctúan entre 6000 a 23000 pies/s (1800 a 7000 m/s aproximadamente). El registro se expresa como el inverso de la velocidad, $\Delta$ t (en el sistema inglés de unidades), medido en microsegundos por pie ( $\mu \mathrm{s} / \mathrm{pie}$ ). En formaciones sedimentarias, la velocidad del sónico depende de varios parámetros; principalmente del material de la matriz de roca (arenisca, caliza, dolomita, etc.) y de la distribución de porosidad.

\section{Rayos Gamma (GR)}

Consiste en la medición de la emisión de rayos gamma proveniente de la radioactividad natural del subsuelo, particularmente de los isótopos radioactivos del potasio, torio y uranio presentes en las rocas y los fluidos. Típicamente, lecturas elevadas de este perfil se dan frente a estratos de arcillas, mientras que frente a otros sedimentos (carbonatos o areniscas) se observan valores bajos. Es un excelente registro de correlación.

Los cuerpos intrusivos pueden ser fácilmente reconocidos en los perfiles de pozo. El perfil acústico es el menos influido por el calibre, lo que lo convierte en uno de los más confiables. Las rocas ígneas se intruyen en secciones pelíticas, aprovechando planos preexistentes de debilidad (estratificación y planos de falla) que favorecen la intrusión. Esta característica resulta en una notable definición en el registro de rayos Gamma.

Las rocas ígneas ricas en potasio tendrán valores más altos de GR.

Los cuerpos ígneos intrusivos del VAM andesíticos y basaltos, los cuales son menos evolucionados desde el punto de vista de la diferenciación magmática, tienen poco feldespato potásico, lo que implica que tendrán valores de GR bajos. En cambio las traquitas que son rocas más evolucionadas presentan valores altos en los registros de GR.

En la figura 6.20 se muestra un ejemplo de los cuerpos ígneos intrusivos en el pozo VAM.x-1 y LRb.x-1 interpretados a partir de los registros del pozo. La respuesta es un aumento de la velocidad y de los rayos gamma. 

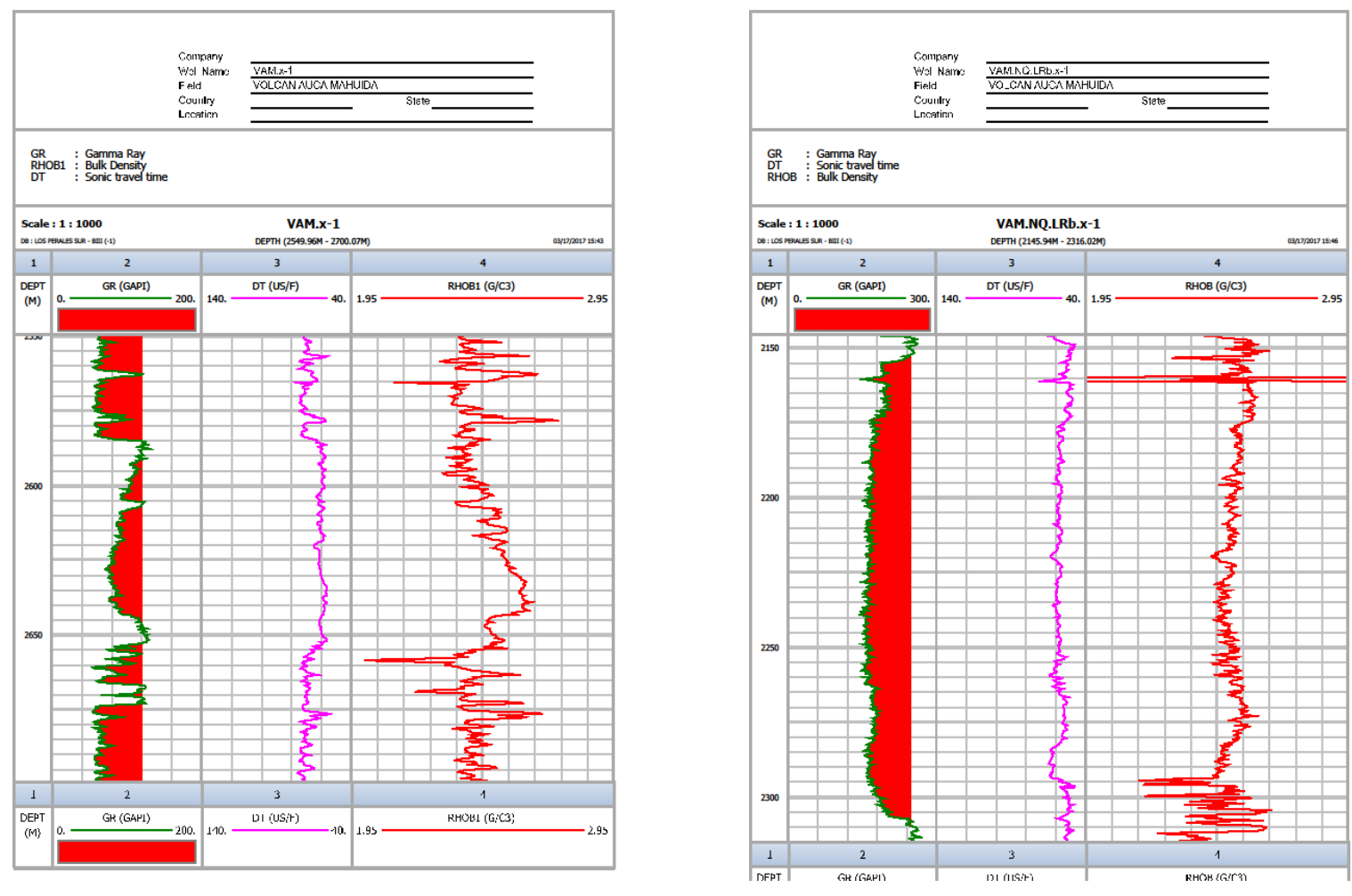

Figura 6.20. Registros de rayos gamma (GR) medidos en grados API, perfil sónico (DT) en $\mu \mathrm{s} / \mathrm{ft}$ y densidad (RHOB) en $\mathrm{g} / \mathrm{cm}^{3}$ en los pozos VAM.x-1 y LRb.x-1. Los intervalos sombreados corresponden a los cuerpos de roca ígnea.

\subsubsection{Interpretación de horizontes y fallas}

La construcción de los sismogramas sintéticos y la interpretración de los horizontes y fallas se realizaron íntegramente en la plataforma Geoframe ${ }^{\mathrm{TM}}$ de Schlumberger.

Se interpretaron los principales horizontes en tiempo (figura 6.21) que luego se convirtieron a profundidad a través de una "pseudo-velocidad" calculada con datos de pozo. La tabla 6.3 muestra la profundidad de los horizontes interpretados en el pozo VAM.x-1 usado para la calibración. Las profundidades expresadas en metros están tomadas desde la topografía, donde MD (Measured Depth) son las profundidades a lo largo de la trayectoria del pozo; TVD (True Vertical Depth) la profundidad proyectada sobre la vertical y TVDSS (True Vertical Depth Sub Sea) la profundidad proyectada en la vertical referenciada al nivel medio del mar. En pozos verticales, MD y TVD coinciden.

La interpretación de los filones volcánicos se realizó siguiendo los valores de alta amplitud presentes en la sísmica para dichos eventos, y la información detallada en los pozos del Apéndice A.

La combinación de secciones del cubo de varianza con las del cubo amplitudes sísmica en tiempo permitió identificar la presencia de las fallas subverticales a verticales, las cuales 
podrían haber sido conductos de alimentación del magma. La varianza es un atributo sísmico que resalta las discontinuidades, como fracturas, saltos de falla, etc. (figura 6.22). Las figuras 6.21 y 6.23 muestran la interpretación sísmica de las principales formaciones geológicas y algunos filones volcánicos identificados.

Tabla 6.3: Profundidades de los principales horizontes sísmicos en el pozo VAM.x-1.

\begin{tabular}{|c|c|c|c|}
\hline Formación & MD $[\mathrm{m}]$ & TVD $[\mathrm{m}]$ & TVDSS $[\mathrm{m}]$ \\
\hline Basalto & 0 & 0 & -1676 \\
\hline Neuquén & 352 & 352 & -1324 \\
\hline Rayoso & 1320 & 1320 & -356 \\
Centenario Sup & 1798 & 1798 & 122 \\
Centenario Inf & 2103 & 2103 & 427 \\
Mulichinco & 2542 & 2542 & 866 \\
Quituco & 2764 & 2764 & 1088 \\
Vaca Muerta & 3140 & 3140 & 1464 \\
\hline Tordillo & 3457 & 3457 & 1781 \\
\hline
\end{tabular}

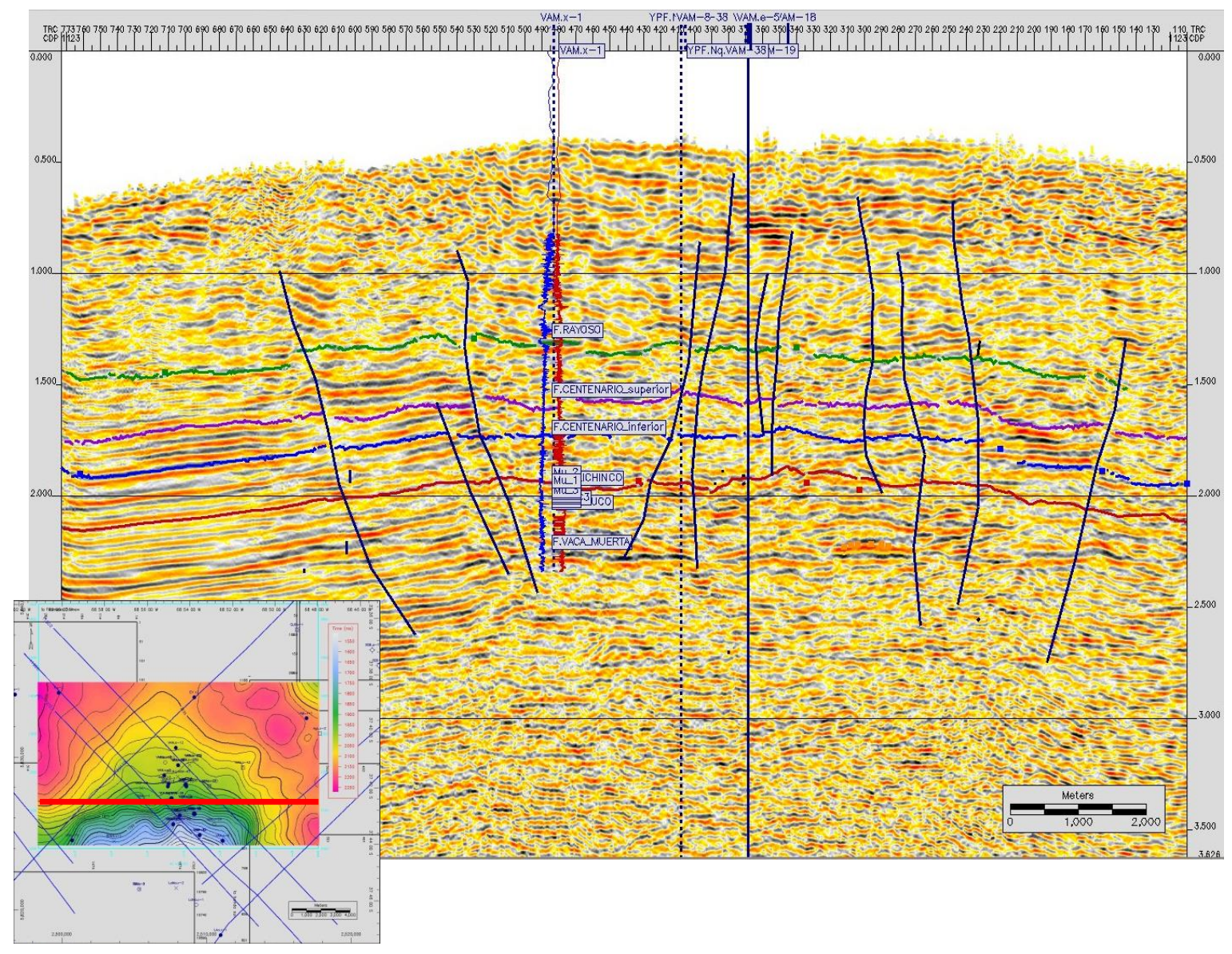

Figura 6.21. Corte sísmico indicado en línea roja en el mapa (margen inferior izquierdo) sobre cubo El volcán 3D. 
El espesor de los filones interpretados consiste en la suma de varios cuerpos más chicos que el método sísmico no puede resolver en forma aislada. Esta interpretación es soportada por la información de los pozos que han atravasado estos cuerpos, por ejemplo el VAM.X-1, VAM.X-2 y VAM.x-3 cercanos al crater central reportado en los legajos de pozo (Apéndice A) y en los trabajos ya mencionados de Bermúdez \& Delpino (1998).

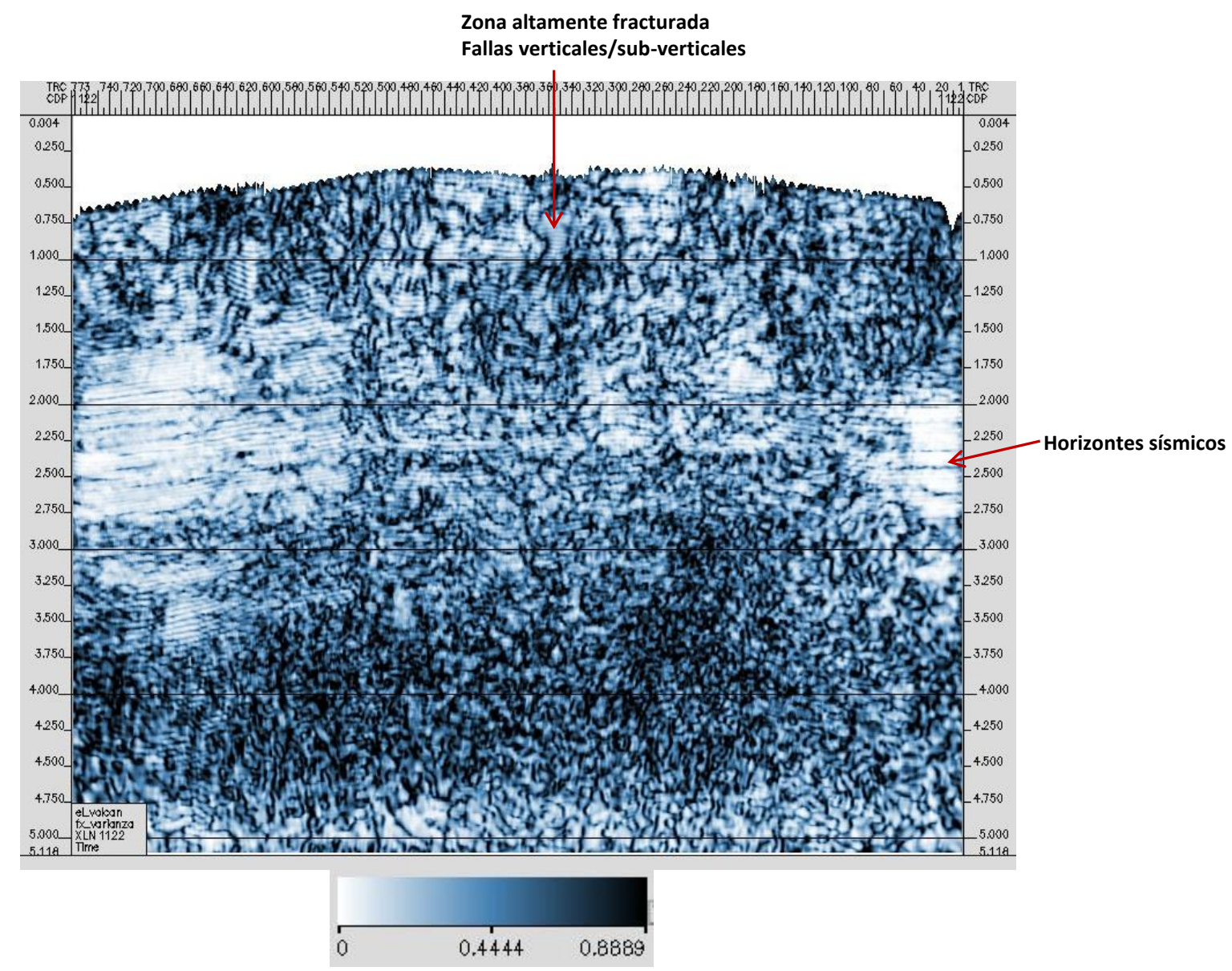

Figura 6.22. Corte sísmico en el cubo de varianza sobre la línea roja del mapa de la figura 6.21. 

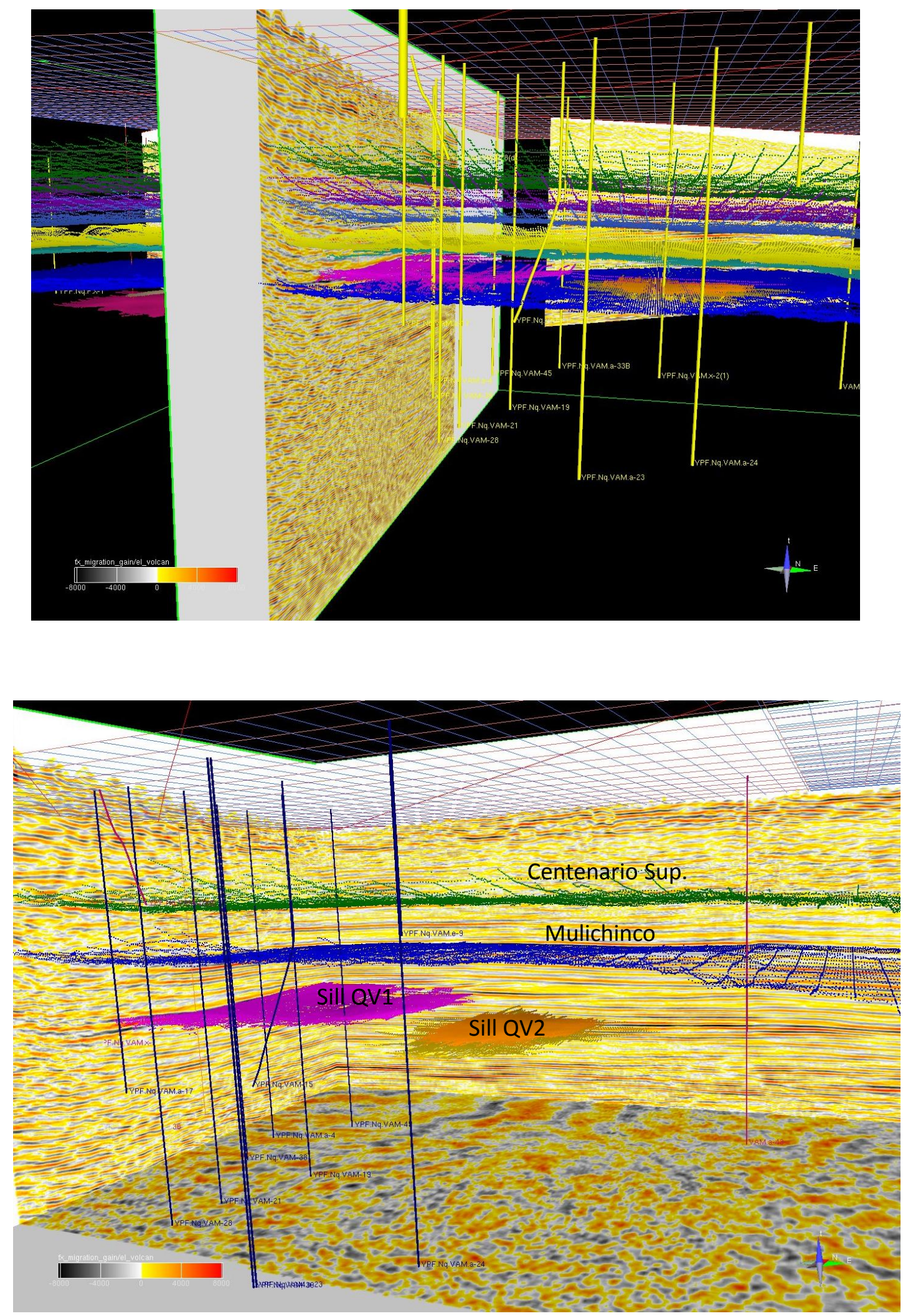

Figura 6.23. Vistas 3D de los principales horizontes sísmicos y dos filones capa, Sill QV1 y Sill QV2, en la formación Vaca Muerta en el cubo de amplitudes (cantidad sin dimensión). 
La figura 6.24 muestra el mapa isócrono de un filón capa para el cual se estimó el espesor promedio. El filón Sill QV1, se observa en el pozo VAM.x-1 con las siguientes profundidades:

TVDSS=1393.4 $\mathrm{m}$ ( $\mathrm{m}$ bajo el nivel del mar)

TVD=MD= $3069.4 \mathrm{~m}$ ( $\mathrm{m}$ bajo boca de pozo)

$\mathrm{GL}=1676 \mathrm{~m}$ (cota del pozo)

Profundidad de la base del pozo $=3480 \mathrm{~m}$

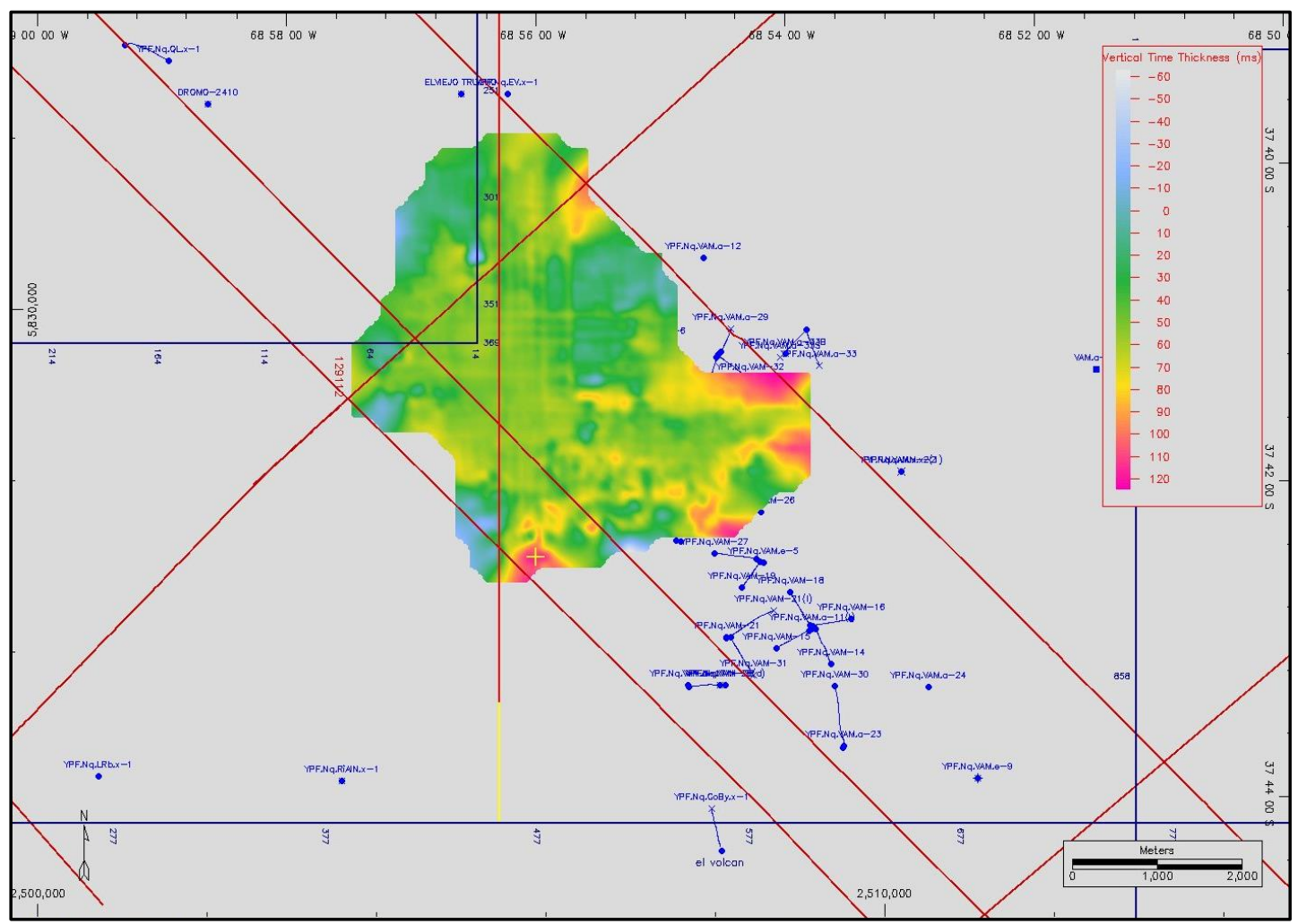

Figura 6.24. Mapa isócrono (en tiempo) resultado de la interpretación sísmica del tope y base del filón Sill QV1.

Dado el gran espesor del filón de referencia interpretado en el cubo sísmico (figura 6.23) se confeccionó un mapa isócoro de espesores en tiempo del mismo.

El espesor promedio del filón es el producto de su espesor en tiempo medido en la sección sísmica y su velocidad. Hay que tener en cuenta que el espesor se mide en tiempo doble. Considerando el espesor promedio en tiempo obtenido de la sección sísmica, 45/2 $\mathrm{ms}=0.0225 \mathrm{~s}$ y su velocidad medida en el perfil sónico del pozo (figura 6.19), $70 \mathrm{us} / \mathrm{ft}$, la velocidad es de $1 / 70 \mathrm{ft} / \mathrm{us}=4354 \mathrm{~m} / \mathrm{s}$. En consecuencia, el espesor promedio del filón interpretado es de aproximadamente $98 \mathrm{~m}(\mathrm{v} \mathrm{t}=4354 \mathrm{~m} / \mathrm{s} 0.0225 \mathrm{~s})$.

El espesor obtenido de la sección sísmica fue interpretado por Bermúdez y Delpino, 
(2002) en pozo VAM.x-1, como fenobasalto aunque de menor espesor. Ellos también reconocen otros cuerpos atravesados en este pozo, cinco intrusivos principales y diez más pequeños. Los cuerpos de mayor espesor se alojan en el Grupo Neuquén, en la Formación Rayoso, en la Formación Centenario Inferior, en Mulichinco y en la Formación Quintuco-Vaca Muerta.

Las traquitas más evolucionadas se intruyen en la Formación Mulichinco en el pozo VAM.x-1 (figura 6.25). Bermúdez y Delpino (2002) analizaron el cutting de estas rocas y concluyen que están enriquecidas en elementos incompatibles, que son los que se concentran en las últimas etapas del proceso de cristalización, entre los que se encuentran el K, Th y U. Esto produce altos valores cercanos a los 200 gAPI en los registros de rayos Gamma (figura 6.20).

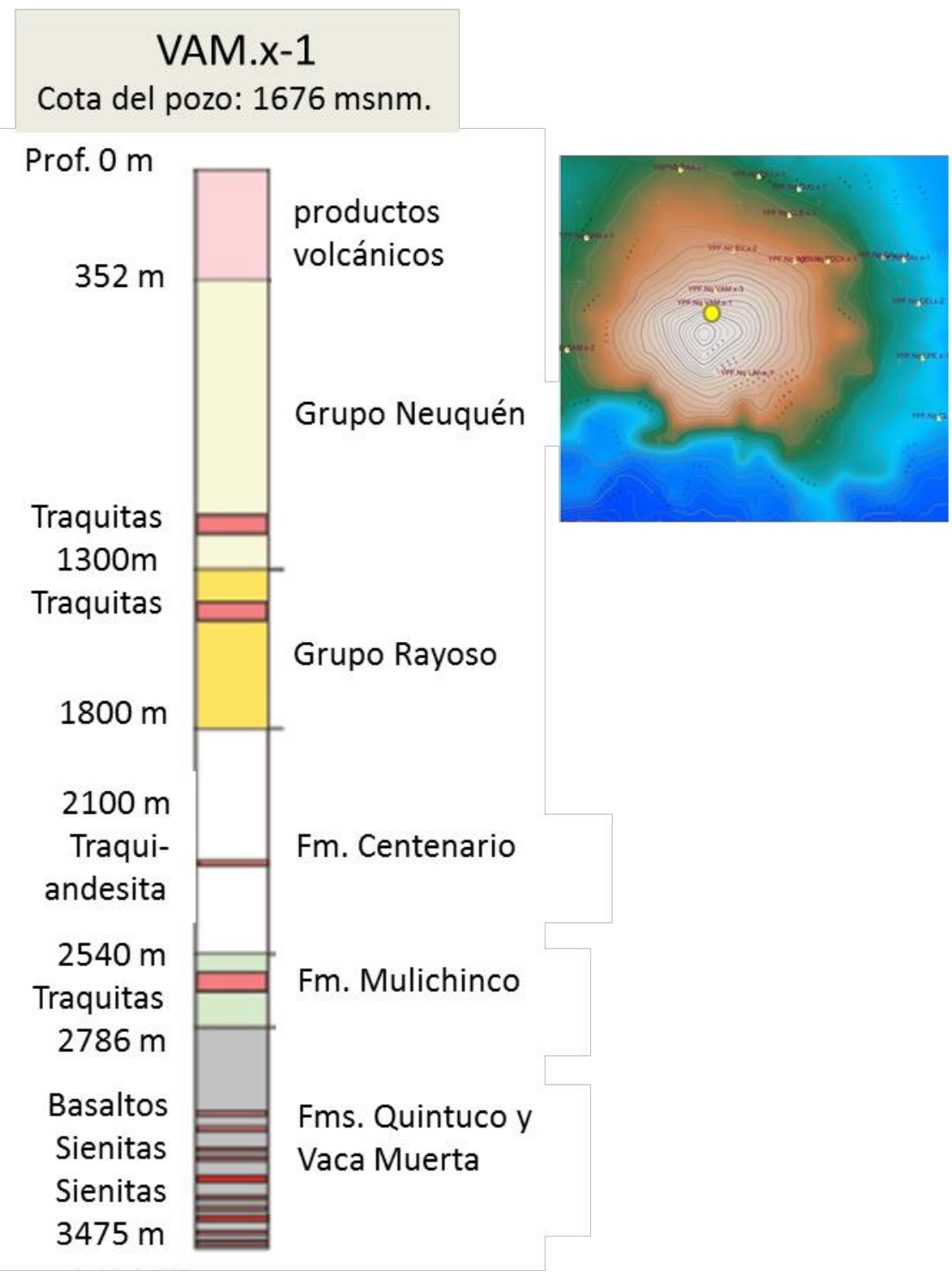

Figura 6.25. Profundidades de los cuerpos intrusivos en el pozo VAM.x.1. Gráfico modificado de Bermúdez y Delpino (2002): descripción litológica a partir del cutting. La cota del pozo está expresada en metros sobre el nivel del mar [msnm]. 


\subsection{Interpretación de los resultados}

En esta sección se describen los principales resultados del presente capítulo.

- Las dimensiones del mapa gravimétrico condicionan la profundidad detectable de las fuentes.

- La anomalía de Bouguer presenta un mínimo relativo en el centro del volcán, el cual se interpreta como una zona de densidad más baja respecto a los valores característicos de rocas ígneas basálticas; además, el colapso del sector puede haber dado lugar a un estado de mayor fracturamiento.

- La presencia de vugs, vesículas, diaclasas y fracturas conducen a que la densidad efectiva sea menor. Esto se debe a que la densidad de grano es distinta a la densidad efectiva de un volumen de roca. Las rocas ígneas intrusivas poseen muy baja o nula porosidad y permeabilidad primarias, por lo que esta propiedad debió ser adquirida después de su emplazamiento. La porosidad secundaria está vinculada a fracturas y a huecos de disolución (vugs). La fracturación está vinculada a eventos tectónicos que afectan a estas rocas y los vugs se originan por circulación de soluciones que provocan la disolución y/o reemplazo de determinados minerales (Comeron, et al., 2002).

La densidad efectiva de un volumen de roca es:

$$
\text { Densidad efectiva }=\text { Dens grano }(1-\phi)+\text { Dens de fluido } \phi
$$

donde $\phi$ es el volumen relativo (poros, vugs, o fracturas) que está ocupado por fluidos.

Suponiendo que el volumen que no es roca está ocupado por agua, se necesita una "porosidad" de $10 \%$ para llevar de una densidad de grano de $2.70 \mathrm{~g} / \mathrm{cm}^{3}$ a una densidad efectiva de $2.53 \mathrm{~g} / \mathrm{cm}^{3}$.

- Por otro lado, las rocas intruidas en el crater del volcán son traquitas que en promedio tienen densidades más bajas que los basaltos. El siguiente cuadro (tabla 6.4, modificada de Wohletz \& Heiken, 1992) detalla valores de densidad y otras propiedades en distintos tipos de rocas ígneas. 
Tabla 6.4. Clasificación de rocas volcánicas según su respuesta en los registros de pozo. Adaptada de Wohletz \& Heiken, 1992.

\begin{tabular}{|c|c|c|c|c|c|}
\hline & Traquitas & Riolitas & Latita & Andesita & Basalto \\
\hline Sílice & $\leftarrow$ & & aumenta & $\leftarrow$ & \\
\hline Aluminio & $\leftarrow$ & & aumenta & $\leftarrow$ & \\
\hline Hierro y Magnesio & & $\rightarrow$ & aumenta & & $\Rightarrow$ \\
\hline Densidad & & $\rightarrow$ & aumenta & - & $\rightarrow$ \\
\hline Radioctividad & $<$ & & aumenta & $\leftarrow$ & \\
\hline
\end{tabular}

- El análisis espectral de los datos gravimétricos indica que los cuerpos analizados están a profundidades muy someras, cuyo resultado es similar al del espectro de datos aeromagnéticos.

- Los principales contrastes de densidad en el volcán Auca Mahuida se presentan entre las formaciones Quintuco-Mulichinco, Tordillo-Auquilco y Grupo Cuyo-basamento Permo-Triásico. Si bien el cubo sísmico no cubre la zona central y sur del volcán, en la porción norte y las laderas se identifican los topes de estas formaciones y se visualizan algunos filones capa intruidos concordantemente en la secuencia sedimentaria como un máximo de amplitud.

- Cuerpos intrusivos con geometría de diques en el cráter y de filones capa en zonas circundantes a éste dan cuenta del ambiente tectónico de la zona donde está emplazado el volcán.

- No se observa una clara correlación entre los datos gravimétricos y magnetométricos. 


\section{CAPÍTULO VII}

\section{MODELADO MAGNÉTICO Y GRAVIMÉTRICO}

El objetivo final de los relevamientos de campos potenciales es obtener información cuantitativa acerca de las fuentes causantes (geometría, dimensiones y profundidad) de las anomalías gravimétricas y magnéticas. Esto puede ser logrado mediante el modelado de las fuentes y contando con valores de propiedades físicas estimados a partir de datos obtenidos en el campo. Si bien existen infinitas soluciones que reproducen los datos observados, el conocimiento de la geología permite poner restricciones a los modelos y por lo tanto disminuir la incertidumbre.

\subsection{Modelado de las anomalías}

El proceso de modelado comprende dos categorías, el método directo (forward) y el inverso.

En los modelos directos 2 y 3D, los valores de los parámetros se estiman en forma iterativa, comenzando con estimaciones guiadas por el criterio geológico del ambiente de estudio. Para esto se construye un modelo inicial de los cuerpos fuente basado en la información disponible del subsuelo. Se calcula la anomalía (magnética o gravimétrica) producida por las fuentes propuestas en el modelo y se la compara con la observada. Luego se ajustan los parámetros del modelo para mejorar el ajuste entre las dos anomalías. Este proceso de tres pasos se repite hasta que se obtiene un parecido aceptable entre la calculada y la observada (Blakely, 1995). Con frecuencia se utilizan los valores obtenidos con el método inverso como restricción de los parámetros. En el presente trabajo se obtuvieron medidas de susceptibilidad magnética de muestras de superficie, las cuales fueron usadas como restricciones adicionales para los valores introducidos en los modelos directos.

El método inverso (3D) utiliza las mediciones magnéticas o gravimétricas para determinar mediante cálculos, parámetros de las fuentes, como por ejemplo la magnetización, así como también forma, profundidad y extensión horizontal de los cuerpos del área de interés.

Tanto el método de modelado directo como el inverso lineal involucran el mismo 
problema de inversión expresado por la matriz genérica del sistema $\boldsymbol{G} \mathbf{m}=\boldsymbol{B}$. Aquí, $\boldsymbol{B}$ es la columna de la anomalía observada, mientras que $\boldsymbol{G} \mathbf{~} \mathbf{m}$ representa al modelo directo asumido por el intérprete para reproducir las observaciones; el cual representa la física del fenómeno y la geometría de las fuentes. El modelo directo consiste de parámetros conocidos o asumidos en la matriz de diseño $\boldsymbol{G}$ y los parámetros desconocidos en $\mathbf{m}$ que deben ser determinados mediante la inversión. El modelado directo evalúa los parámetros desconocidos en m mediante prueba y error, mientras que el modelo inverso obtiene un estimador objetivo de las variables desconocidas a través de la solución por mínimos cuadrados expresada como $\mathbf{m}$ $=\left(\boldsymbol{G}^{\mathrm{T}} \boldsymbol{G}\right)^{-1} \boldsymbol{G}^{\mathrm{T}} \boldsymbol{B}$. Por lo tanto, la inversión estima los parámetros desconocidos (m) del modelo directo a partir de sus parámetros asumidos o conocidos $(\boldsymbol{G})$ y los valores de anomalías medidas $(\boldsymbol{B})$.

\subsection{Formulación del problema inverso- Caso magnético}

En general, la magnetización $\boldsymbol{M}$ es una función de la posición, donde la dirección y magnitud pueden variar desde un punto a otro, esto es, $\boldsymbol{M}=\boldsymbol{M}(\boldsymbol{r})$, donde $\boldsymbol{r}$ es la posición de un diferencial de volumen, $d r^{3}$. No se hace ninguna suposición sobre si la magnetización es inducida o remanente. El campo magnético $\boldsymbol{B}$ en un punto $\boldsymbol{r}_{j}$ resultante de un volumen $V$ que contiene la magnetización $\boldsymbol{M}(\boldsymbol{r})$ está dado en el sistema cgs por (ec. 7.1):

$$
\boldsymbol{B}\left(\boldsymbol{r}_{j}\right)=\nabla \int_{V} \boldsymbol{M}(\boldsymbol{r}) \cdot \nabla \frac{1}{\left|\boldsymbol{r}-\boldsymbol{r}_{j}\right|} d r^{3}
$$

Esta expresión corresponde a un modelo de distribución de dipolos y muestra que el vector magnetización $\boldsymbol{M}(\boldsymbol{r})$ es el parámetro natural para la inversión, lo cual es una observación crucial.

El campo magnético depende linealmente de la magnetización, y la estimación de la magnetización a partir del campo magnético es un "problema inverso lineal". Si en cambio, se calculara alguna propiedad de $V$ el problema sería "inverso no-lineal".

Si el volumen $V$ se subdivide en un conjunto de dominios $v_{k}$, cada uno con magnetización constante $\boldsymbol{M}_{k}$, entonces:

$$
B_{\beta}\left(\boldsymbol{r}_{j}\right)=\sum_{k, \alpha}^{N, 3} M_{k, \alpha} \int_{v_{k}} \partial_{\alpha} \partial_{\beta} \frac{1}{\left|\boldsymbol{r}-\boldsymbol{r}_{j}\right|} d r^{3}
$$

donde $M_{k, \alpha}(\alpha=1,2,3)$ son las componentes cartesianas del vector de magnetización $\boldsymbol{M}_{k} \mathrm{y} B_{\beta}$ 
$(\beta=1,2,3)$ son las componentes cartesianas del vector $\boldsymbol{B}$.

La ecuación (7.2) define el problema directo: dado un conjunto de fuentes $\boldsymbol{M}_{k}(k=1, \ldots$, $N$ ), $\boldsymbol{B}_{\mathrm{j}}$ es el campo de anomalía magnética predicha en los puntos $\boldsymbol{r}_{j}(j=1, \ldots, P)$. Debido a que se suma sobre el índice de coordenada $\alpha$, se tiene libertad para elegir el sistema de coordenadas que resulte más conveniente para los cálculos. También surge que una cantidad invariante con la coordenada, tal como la amplitud $M(\boldsymbol{r})=|\boldsymbol{M}(\boldsymbol{r})|$ podrá ser determinada en forma robusta a partir de los datos.

La ecuación anterior se puede representar en forma más concisa como (ec. 7.3):

$$
\boldsymbol{B}=\boldsymbol{G} \boldsymbol{M}
$$

El problema inverso para hallar el vector magnetización consiste en resolver $\boldsymbol{M}$ dado $\boldsymbol{B}$, sujeto a una condición de regularización adecuada. La regularización es una forma de poner restricciones al modelo de modo de mantenerlo lo más simple posible, en el sentido de que tenga la menor estructura compatible con un ajuste aceptable de los datos. Este tipo de restricción, que implica eliminar explícitamente la complejidad innecesaria (y generalmente poco realista) de los modelos, se incluye en el término de la norma del modelo (Constable et al., 1987). El error total a minimizar es entonces la suma de las dos normas, pesada por un factor de regularización. Sin pérdida de generalidad se puede elegir la regularización de mínimo gradiente de Tikohonov (Tikohonov et al., 1995). El problema inverso consiste entonces en hallar $\boldsymbol{M}$ que minimice la función objetivo en (ecs. 7.4-7.6):

$$
\begin{gathered}
\emptyset(\boldsymbol{M})=\emptyset_{d}(\boldsymbol{M})+\lambda \emptyset_{m}(\boldsymbol{M}) \\
\emptyset_{d}(\boldsymbol{M})=\frac{1}{P} \sum_{j=1}^{P}\left|\frac{\boldsymbol{G}_{j} \boldsymbol{M}-\boldsymbol{B}_{j}}{e_{j}}\right|^{2} \\
\emptyset_{m}(\boldsymbol{M})=\frac{1}{N} \sum_{i=1}^{N} \sqrt{\left(m_{i}^{\text {ref }}-m_{i}^{\text {pre }}\right)^{2}}
\end{gathered}
$$

En la ecuación (7.4), $\varnothing(\boldsymbol{M})$ es la suma de un término de datos $\emptyset_{d}(\boldsymbol{M})$ y un término de modelo $\emptyset_{m}(\boldsymbol{M})$ con un parámetro de regularización de Tikohonov, $\lambda$. La ecuación (7.5) define la función objetivo de los datos como la diferencia cuadrática entre la anomalía calculada y la 
observada, pesada por la inversa del error $e_{j}$. Para este término de la función objetivo se suele adoptar el valor 1, porque el valor esperado de la sumatoria en la ecuación (7.5) en el caso en que las observaciones son independientes y su incerteza responde a una distribución Gaussiana de media cero, es igual a $P$, el número de observaciones. Además, se sabe que los modelos correspondientes a los menores valores posibles de $\emptyset_{d}(\boldsymbol{M})$ carecen de sentido físico (Constable et al., 1987). La ecuación (7.6) define la "norma" del modelo y cuantifica cuánto se parece el modelo predictivo $m^{\text {pre }}$ al modelo de referencia $m^{\text {ref }}$ (un tipo de modelo preferido en base al conocimiento geológico del susbsuelo). Esta sumatoria debe acercarse a cero para que el modelo predictivo honre la geología. Adicionalmente, otras restricciones y condiciones de borde, tales como cotas superior e inferior, pueden especificarse para $\boldsymbol{M}$ de acuerdo con el problema en cuestión.

El problema inverso consiste entonces en optimizar la proximidad del modelo predictivo y su respuesta (calculada en forma directa) con los datos observados y con aquello a lo que el intérprete supone que el modelo debe parecerse. En la práctica, el proceso de inversión busca balancear estas dos cantidades (ajuste de los datos observados lo más cerca posible del modelo geológico de referencia) para llegar a un modelo final. El algoritmo de inversión busca un modelo que minimice la función objetivo $\emptyset(\boldsymbol{M})$ con la condición $\emptyset_{d}(\boldsymbol{M}) \cong 1$ (figura 7.1).

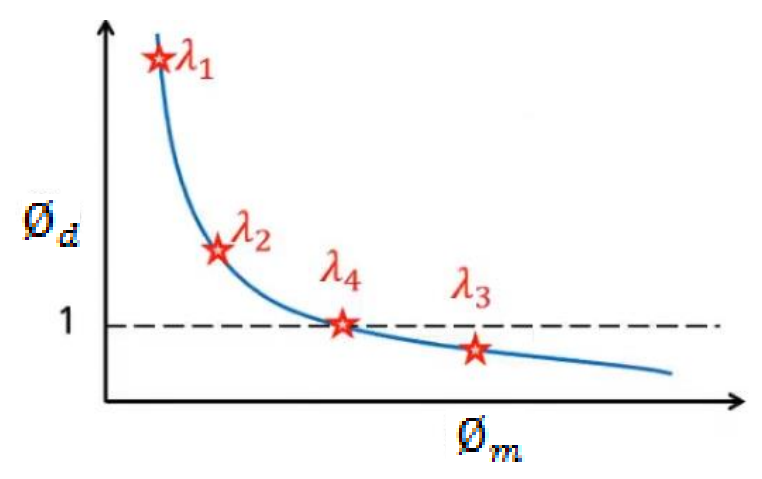

Figura 7.1. Esquema del proceso de inversión.

\subsubsection{Aplicación a los datos magnéticos del VAM}

En la mayoría de los métodos de inversión se hace la suposición de que la respuesta magnética se origina en la magnetización inducida. Cuando esto se cumple, tales métodos permiten obtener modelos confiables. Sin embargo, si la remanencia es importante las inversiones basadas en la suposición de que toda la magnetización es inducida no lograrán reproducir las anomalías observadas en forma aceptable. En cambio, se obtendrán modelos más realistas modelando la magnetización como un vector y permitiendo que adopte una dirección arbitraria, es decir que se tiene también en cuenta la remanencia. La inversión del 
vector magnético (MVI por sus siglas en inglés; Aisengart, 2015) es una técnica de modelado inverso como se describió en la sección anterior, con la ventaja de que tiene en cuenta ambas magnetizaciones, inducida y remanente sin tener conocimiento previo de la dirección de esta última (MacLeod \& Ellis, 2013). Por el contrario, la inversión convencional de susceptibilidad se basa en la premisa de que todos los dominios magnéticos de las rocas se orientan en forma paralela al campo geomagnético. En ambos casos, inversión de susceptibilidad convencional y MVI, el resultado es un modelo de la distribución de magnetización del subsuelo. El método convencional presenta las limitaciones mencionadas, mientras que el MVI resulta en modelos que representan mejor las estructuras de roca magnetizada del subsuelo cuando los efectos de remanencia y anisotropía son significativos.

La aplicación de MVI devuelve el vector $\boldsymbol{M}$ (magnitud y dirección) y, a partir de él, tres cantidades escalares que son importantes para la interpretación del modelo de inversión: la amplitud del vector, la componente de $\boldsymbol{M}$ en la dirección del campo geomagnético, $E_{\text {proy, }}$ y la proyección de $\boldsymbol{M}$ en el plano perpendicular a la dirección del campo geomagnético, $E_{\text {perp }}$ (figura 7.2).

En la MVI de VOXI Earth Modelling, $|\boldsymbol{M}|$ se normaliza por la intensidad del campo terrestre, por lo cual tiene dimensiones de una susceptibilidad.

Se puede escribir el versor $U$ en la dirección del campo IGRF, y $V, W$ los ortogonales al mismo y entre ellos, los cuales cumplen la regla de la mano derecha. De este modo $E_{\text {perp }} \mathrm{y} E_{\text {proy }}$ quedan definidos como (ec. 7.7):

$$
\begin{gathered}
E_{\text {perp }}=\sqrt{V^{2}+W^{2}} \\
E_{\text {proy }}=U
\end{gathered}
$$

$E_{\text {perp }}$ será siempre positivo mientras que $E_{\text {proy }}$ podrá ser positivo o negativo como se indica a continuación.

Zonas con valores grandes de $E_{\text {perp }}$ indican magnetización remanente o anisotropía magnética importantes, mientras que valores pequeños de esta componente indican que la magnetización es principalmente inducida, o bien reversa (esta última característica se traduciría además en un $E_{\text {proy }}$ negativo y con valor absoluto significativo). 


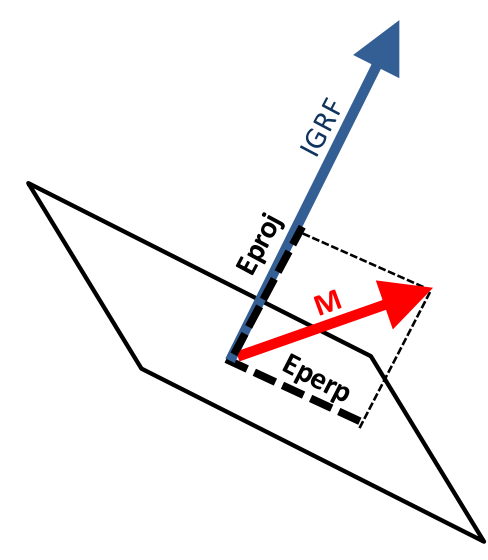

Figura 7.2. Diagrama con las componentes obtenidas en el modelo inverso MVI.

Dado que en el área de estudio predomina la magnetización remanente, se aplicó la técnica de MVI a los datos de la anomalía TMI (sin reducir al polo) y se obtuvo un modelo 3D de magnetización (pseudo-susceptibilidad) usando VOXI Earth Modeling de Geosoft ${ }^{\mathrm{TM}}$. La elevación en el modelo varía entre 2175 y -5038 metros (con $z$ positivo sobre el nivel del mar). La grilla construida para procesar los datos tiene un tamaño de celda de $500 \mathrm{~m}$ en las direcciones $x$ e y, y un tamaño vertical de $465 \mathrm{~m}$ en la dirección $z$. Con estos parámetros se obtuvo un volumen de magnetización con 85 celdas en la dirección de $x, 74$ en $y$, y 61 en $z$ (figura 7.3). El modelo convergió en 12 iteraciones en 20 minutos.

La diferencia entre los datos observados y el resultado del modelo predictivo, produce valores pequeños (figura 7.4). Esto indica que se logró un buen ajuste entre el modelo inicial y el modelo final.

Se exportó el voxel vectorial a una base de datos, con lo cual se pudo obtener las componentes de amplitud de las tres direcciones $M_{x}, M_{y}$ y $M_{z}$. El rango de amplitudes obtenido está entre 0.000039 y 0.045136 SI (figura 7.5).

La figura 7.6 a) muestra la isosuperficie de 0.012 SI de la inversión del vector magnético (formas en celeste rodeando las áreas centrales) junto con la grilla de la topografía y b) señal analítica; en c) una sección E-O de la misma isosuperficie, en la cual se observa que los cuerpos se extienden en profundidad hasta unos $3.3 \mathrm{~km}$ bajo nivel medio del mar. La figura 7.7 corresponde a una sección en planta de la amplitud del MVI junto con la amplitud de la señal analítica derivada de los datos de anomalía TMI del VAM. Se puede observar una buena correspondencia entre las ubicaciones de las fuentes y el mapa de señal analítica (figura 7.7). Un comportamiento similar se observó en otros estudios, y se lo interpretó como indicador de consistencia geológica (MacLeod \& Ellis, 2013).

Los valores de la proyección de $\boldsymbol{M}$ en el plano perpendicular a la dirección del campo geomagnético, $E_{\text {perp }}$ obtenidos en la inversión son mayores que los de la componente de $\boldsymbol{M}$ en la dirección del campo geomagnético, $E_{\text {proy, }}$ esto es una herramienta más para reafirmar que la componente remanente es la que domina (figura 7.8). 


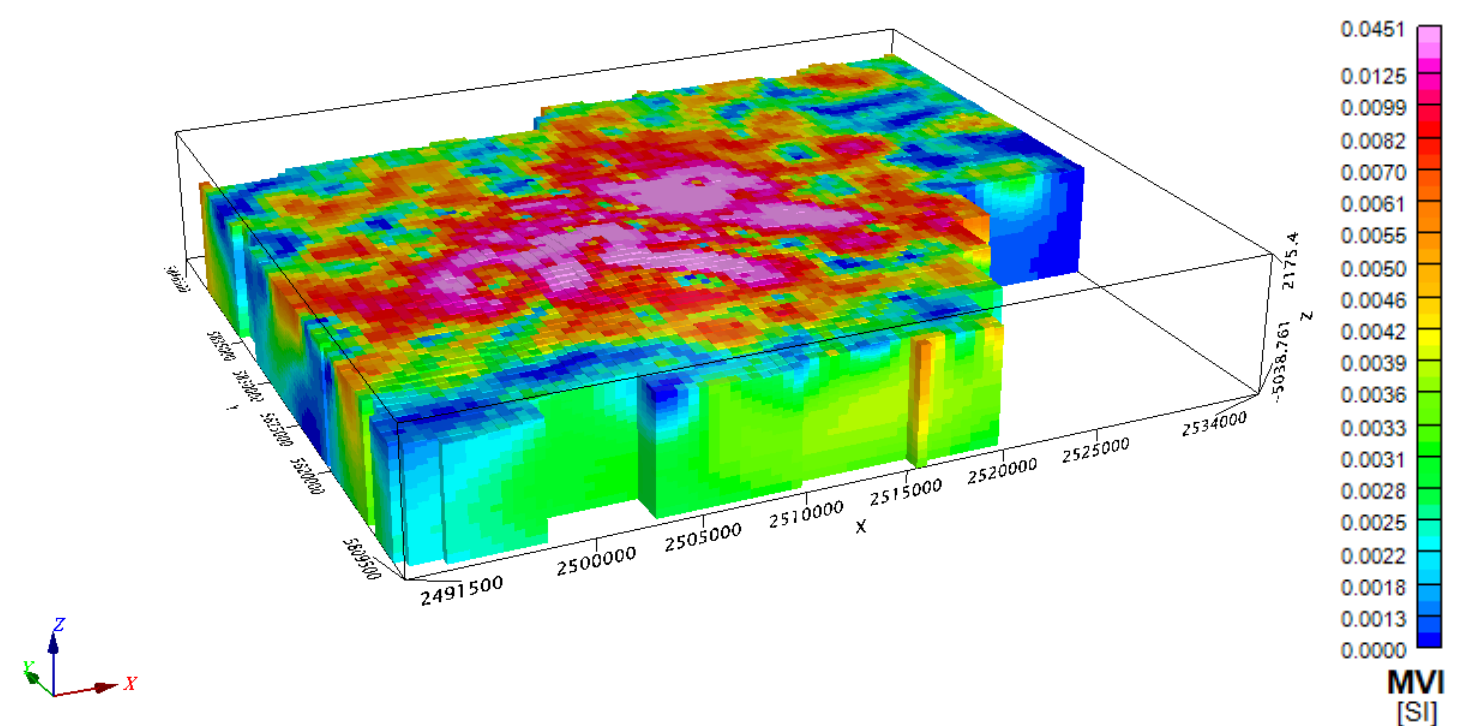

Figura 7.3. Volumen de magnetización obtenido en el modelo de inversión MVI.

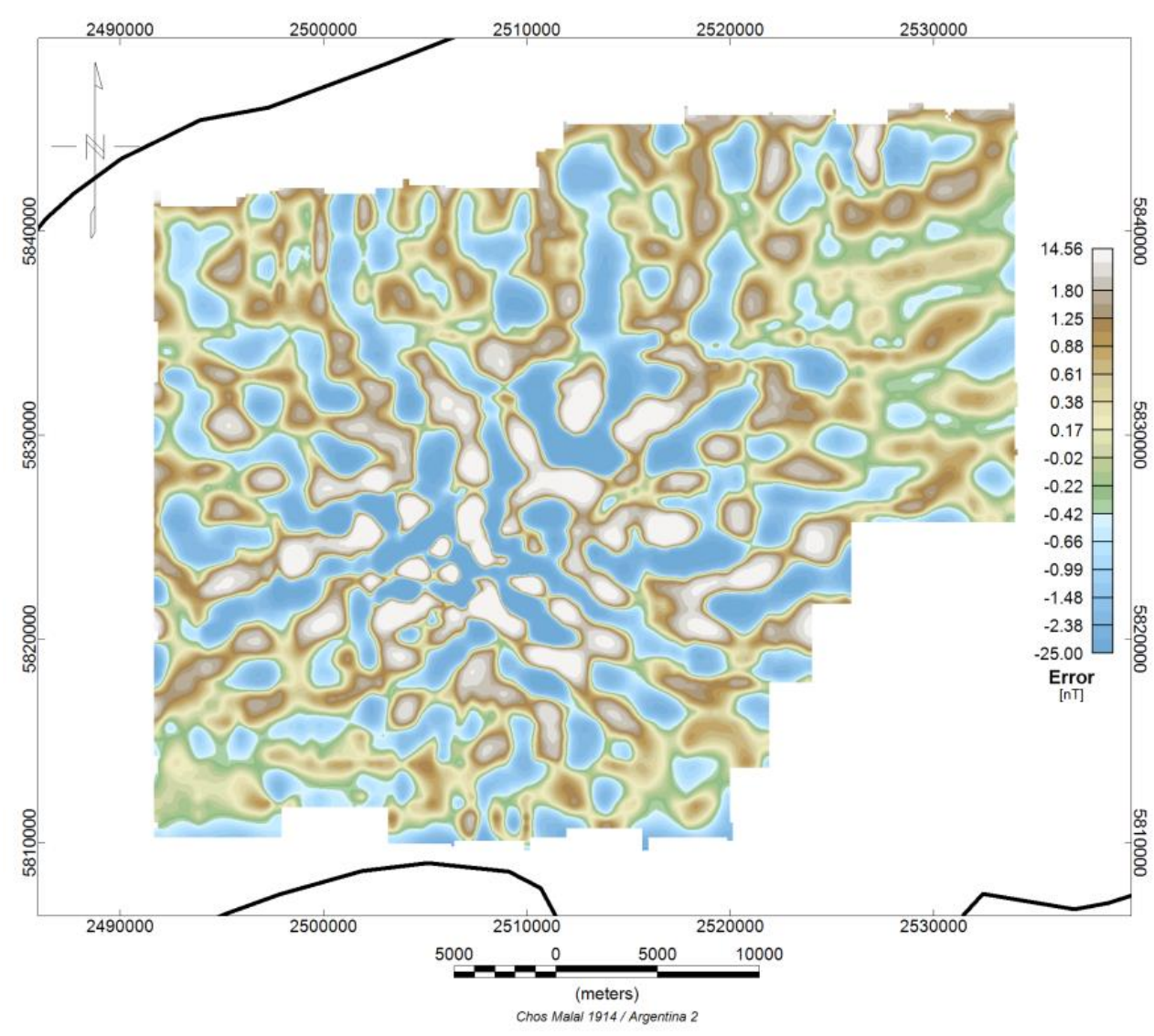

Figura 7.4. Diferencia entre los datos observados y el resultado del modelo predictivo. 


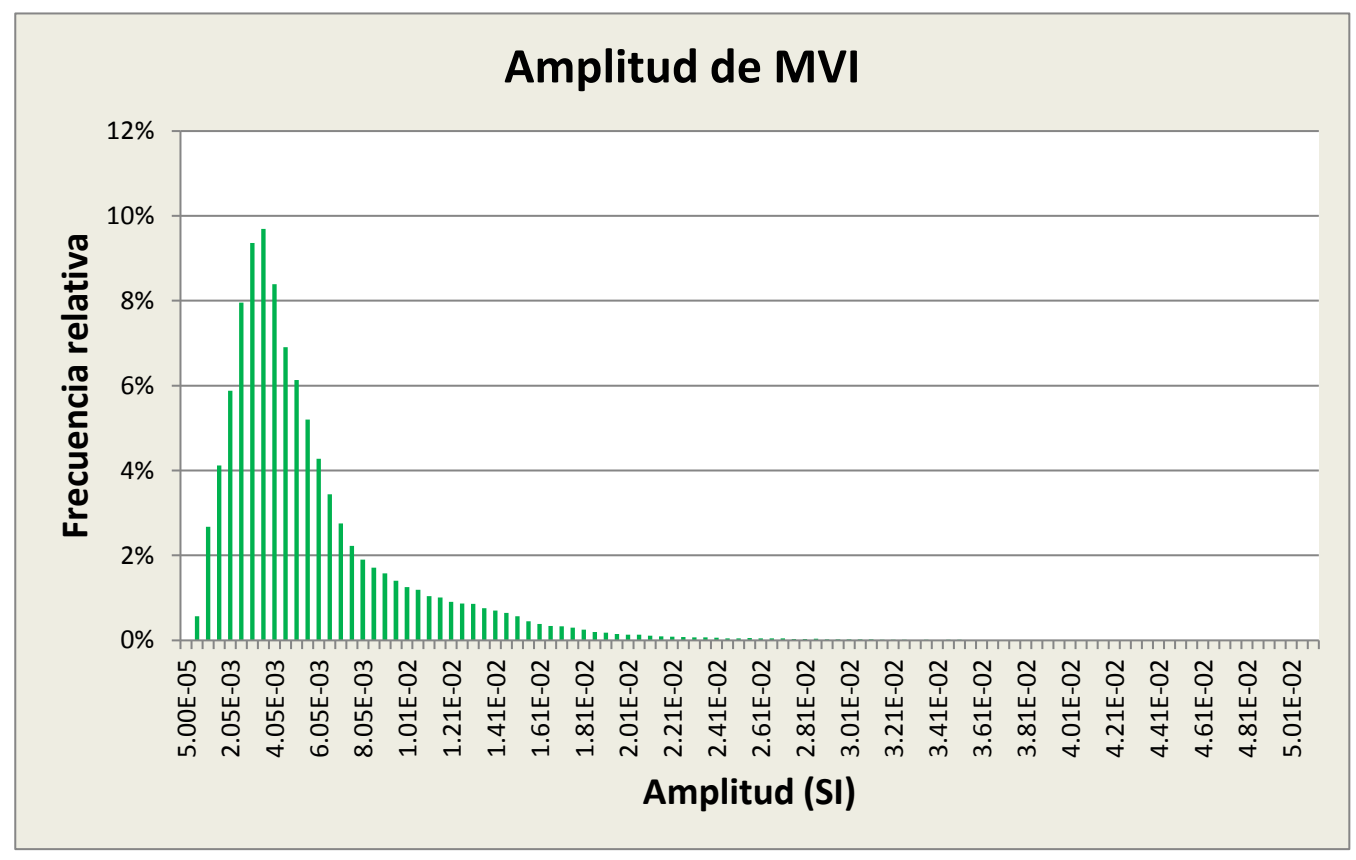

Figura 7.5. Histograma de amplitudes de la magnetización obtenida del MVI.
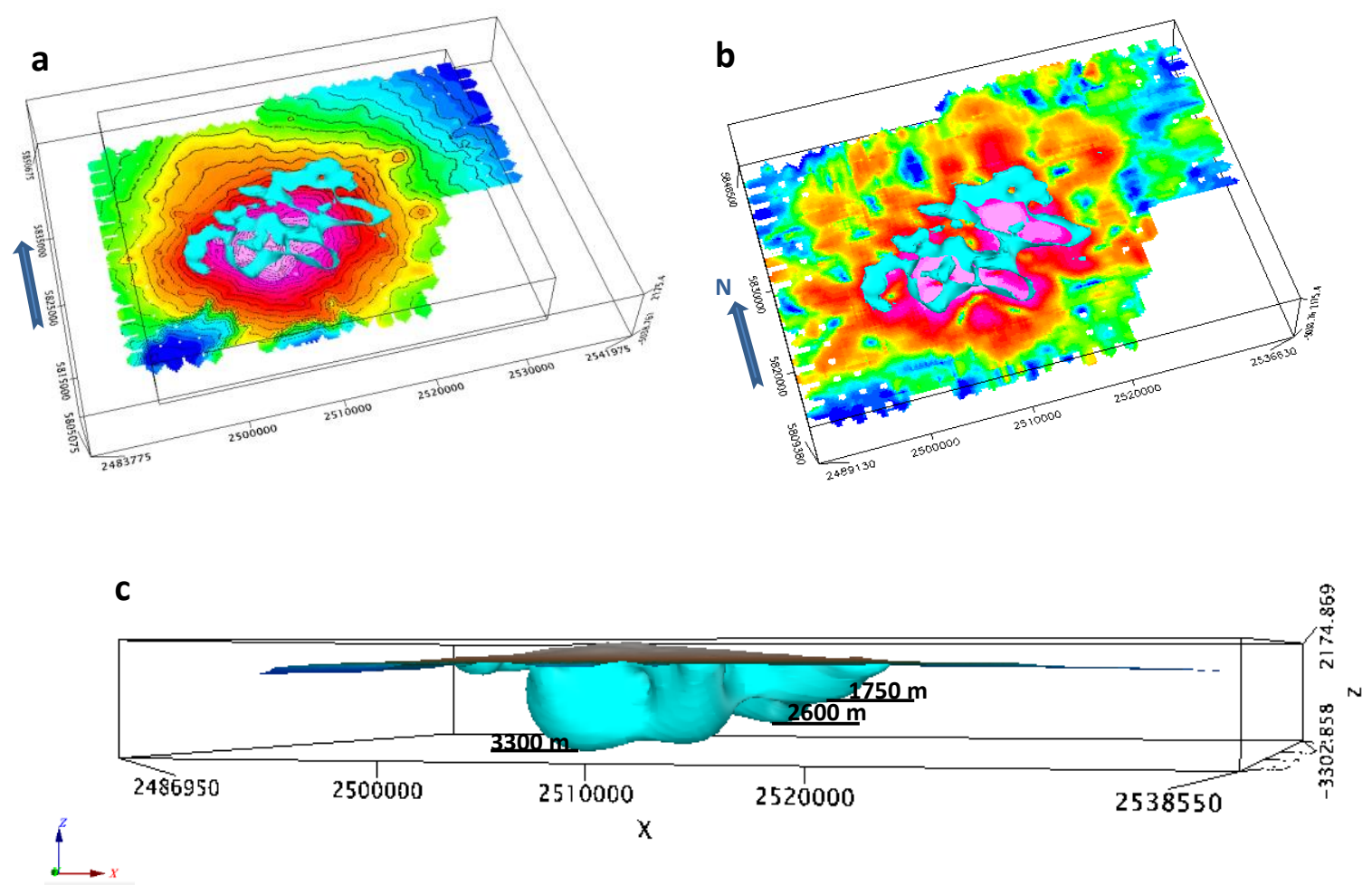

Figura 7.6. Isosuperficie de 0.012 SI que representa los cuerpos fuente de anomalía magnética (color celeste) sobre la topografía a); b) la misma isosuperficie que en a) sobre el mapa de señal analítica. c) Geometría de la isosuperficie de 0.012 SI en el cráter central del volcán. La superficie en el tope es la topografía. La dirección de $z$ es positiva arriba del nivel medio del mar. 

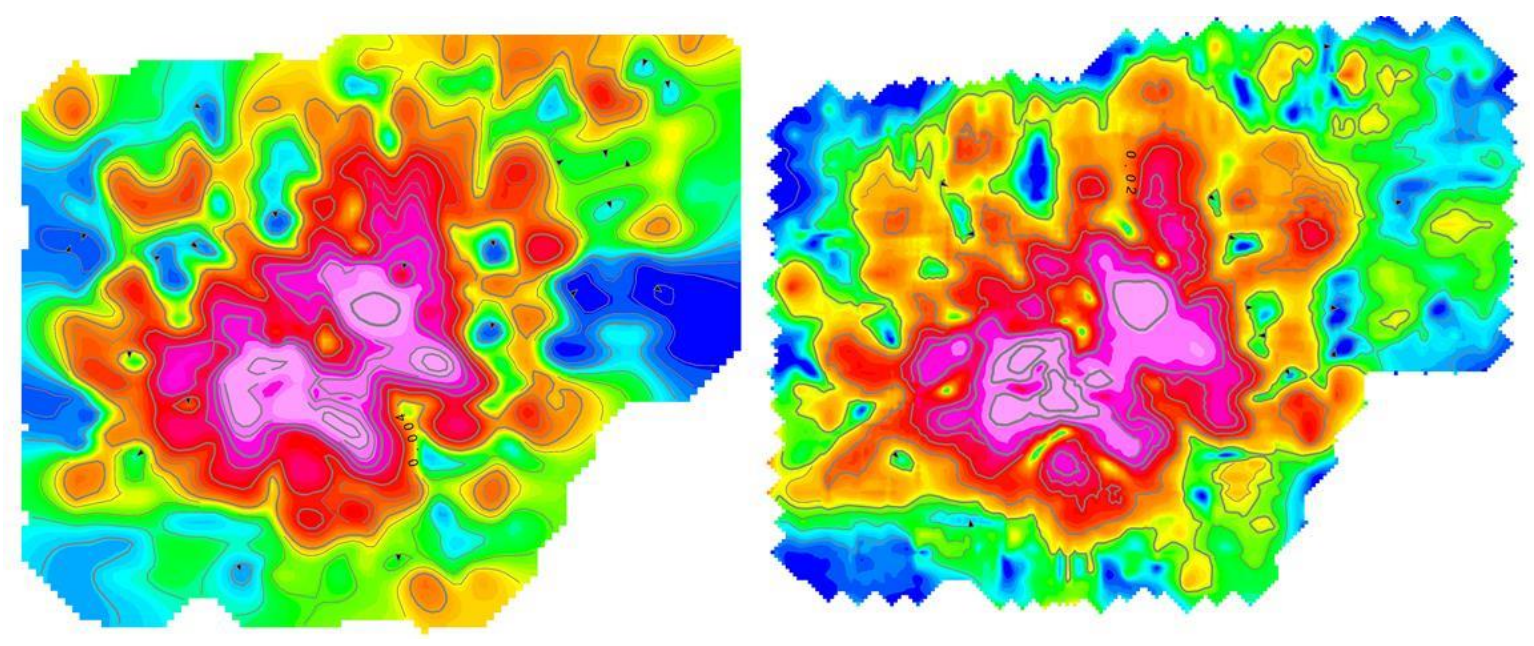

Figura 7.7. Amplitud del MVI y señal analítica en planta.

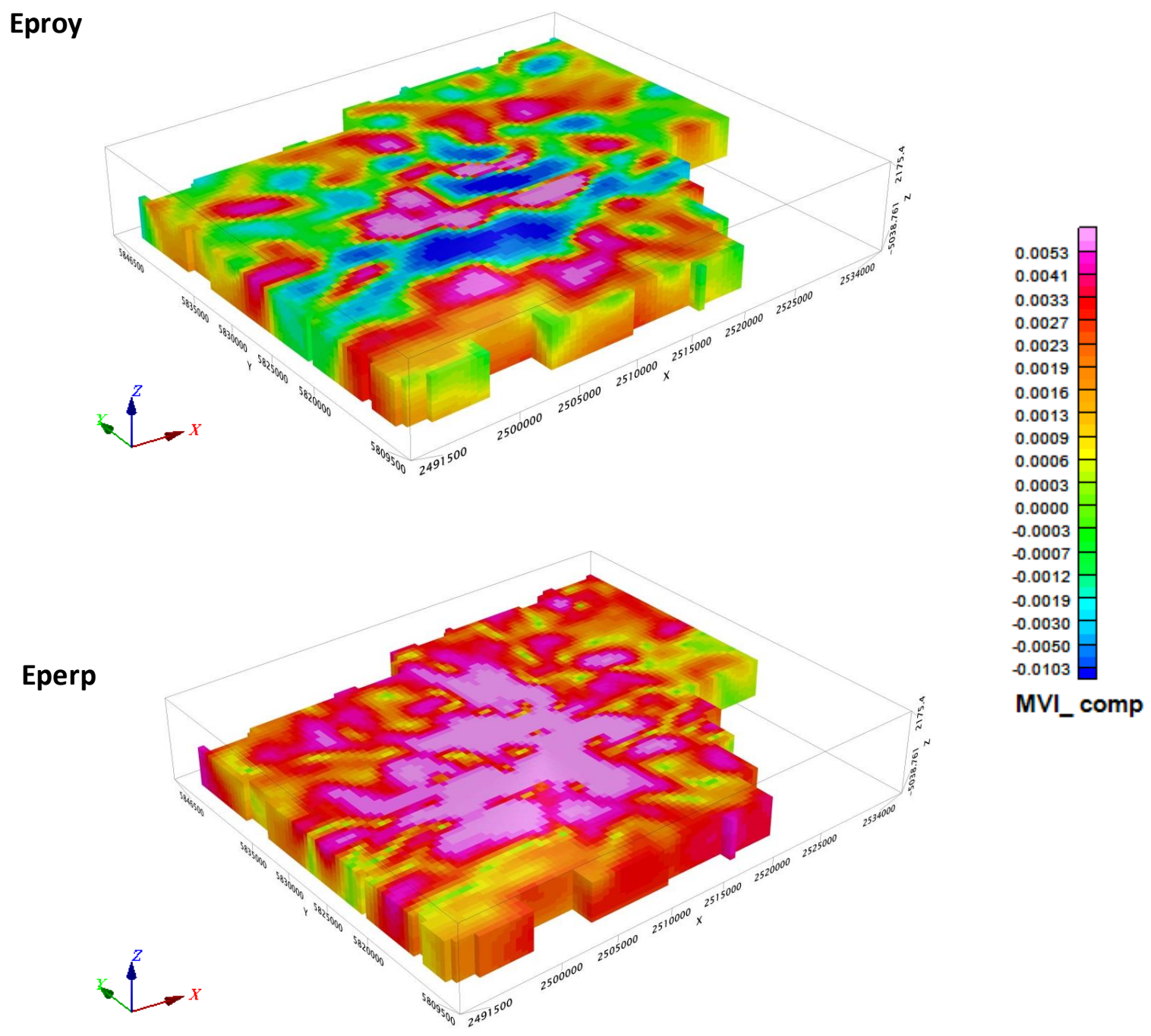

Figura 7.8. Componente proyectada Eproy y perpendicular Eperp de la inversión MVI. 


\subsection{Modelado directo 2.75D}

El modelado bidimensional normalmente se realiza sobre perfiles atravesando lóbulos positivos y negativos de la anomalía, trazados en dirección perpendicular a los contornos de la misma (es decir en direcciones en las cuales el gradiente de la anomalía es significativo). En el caso en que se use la RTP (López-Lorea et al., 2011), como en el presente trabajo, se busca que el perfil incluya un máximo o mínimo. Cuando el corte geológico se extiende hasta el infinito sin variación en la dirección perpendicular, el proceso se describe como modelado bidimensional (2D). Se habla de modelado "dos y medio" dimensional (2.5D) cuando el tamaño de las fuentes en la dirección perpendicular al perfil es finito y es el mismo hacia uno u otro lado del perfil. Cuando estos bordes de las fuentes se ubican a distancias distintas del perfil, o el cuerpo fuente está inclinado respecto a la dirección del perfil, se conoce como modelado "dos y tres cuartos"-dimensional (2.75D).

El modelado 2.75D puede proveer soluciones razonables si está soportado por datos confiables y modelos geológicos previos. No obstante, una limitación para la construcción de modelos geológicos en general, es la carencia o escasez de datos de subsuelo que conduce a tener que extrapolarlos a partir de información medida en superficie. Aún usando información proveniente de perforaciones, se tiene la desventaja de que se dispone en forma local.

\subsubsection{Modelado magnético de las anomalías del VAM}

Es importante tener en cuenta que el objetivo final del modelado magnético es obtener información sobre la geometría y profundidad de las fuentes causantes de las anomalías principales restringiendo los valores de magnetización y susceptibilidad magnética dentro de un intervalo basado en datos paleomagnéticos o petrológicos (Blanco et al., 2006). Se considera que los sedimentos que forman parte de la secuencia sedimentaria son no-magnéticos (Blanco et al., 2003) mientras que la respuesta anómala obtenida, es producto de rocas ígneas en forma de diques, filones capa y coladas basálticas de superficie.

La estrategia seguida para modelar el campo de anomalías magnéticas del Auca Mahuida está relacionada a la complejidad del mismo. El primer factor de complejidad radica en la coexistencia de fuentes magnetizadas en campos directos e invertidos; el segundo se relaciona con el hecho de que dichas fuentes son muy cercanas unas de otras, en ocasiones superpuestas y agrupadas. Esta "coalescencia" de los efectos magnéticos de fuentes cercanas determina el campo de anomalías resultante. Las fuentes se ubican principalmente debajo del cráter central y las anomalías presentan un rango de longitudes de onda entre $1.5 \mathrm{~km}$ y $6 \mathrm{~km}$ aproximadamente. Como se vio en el Capítlulo V, la profundidad de las fuentes detectables depende de los parámetros geométricos que definen la grilla aeromagnética, por ejemplo la altura de adquisición y la resolución espacial de los datos. En el caso de estudio, las longitudes de onda del campo de anomalías están asociadas a cuerpos ubicados a 
profundidades someras e intermedias dentro de la secuencia sedimentaria, hasta $3.5 \mathrm{~km}$ (la profundidad del tope del basamento en este sector de la cuenca es de unos 5 a $6 \mathrm{~km}$ ).

La elección de los perfiles se hizo sobre el mapa de anomalías magnéticas reducido al polo (Salem \& Ali, 2016; Shirman, et, al., 2015) con el propósito de resolver la estructura situada debajo del edificio central del volcán (figura 7.9). Estos modelos se ajustaron con los resultados obtenidos de la inversión magnética 3D (figura 7.10), los cuales permitieron acotar la extensión lateral y profundidad de los cuerpos usando como guía una sección del modelo tridimensional correspondiente al plano del perfil.

Los modelos se realizaron con el programa GM-SYS de Oasis Montaj" ${ }^{\mathrm{TM}}$, versión 8.2, que ofrece una implementación de modelado directo 2.75D. Los cálculos que realiza están basados en las expresiones obtenidas por Talwani - Heirtzler (1964) (Apéndice B) y el algoritmo propuesto por Won \& Bevis (1987). El programa permite cambiar las propiedades a lo largo de la dirección perpendicular a la traza del perfil $-y,+y$ (figura 7.11). Se debe considerar no sólo el efecto de la fuente que está directamente en el plano del perfil sino también el efecto lateral de la anomalía a poca distancia, teniendo en cuenta que los efectos de las fuentes vecinas se superponen. En este sentido, la ventaja de los modelos 2.75D reside en que permiten restringir la magnetización a los cuerpos fuente, porque de lo contrario (modelos 2D) se estaría considerando un cuerpo más extendido a lo largo de la dirección de $y$ de lo que en realidad es (si bien a lo largo de y el efecto de la fuente se atenúa). En estos modelos simples (figuras 7.12- 7.14) se logró un buen ajuste entre la anomalía observada y calculada, usando los valores de susceptibiliad magnética y magnetización descriptas en las tablas 7.1-7.3. 


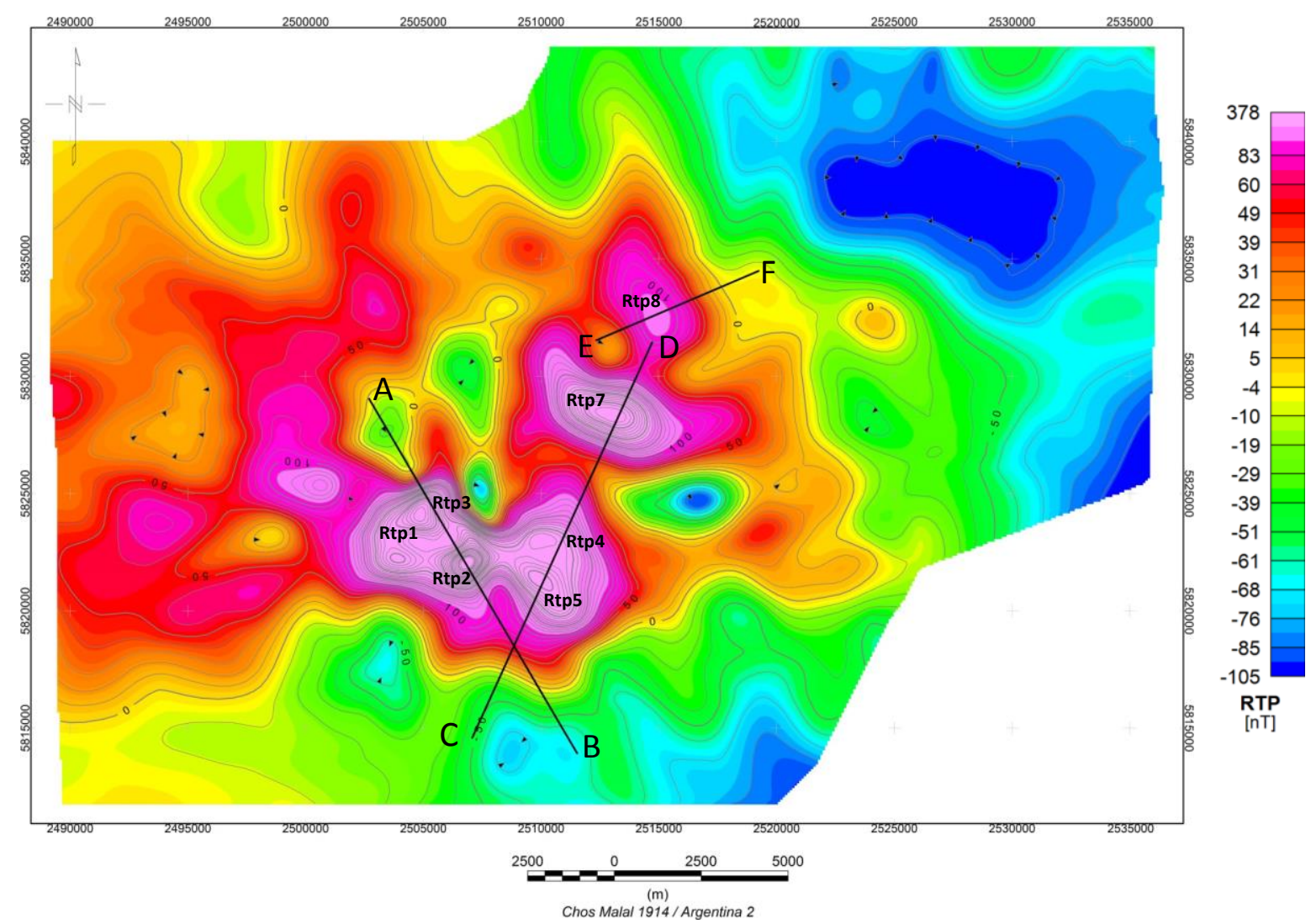

Figura 7.9. Perfiles AB, CD y EF sobre el mapa de anomalías reducido al polo con los efectos de magnetización inducida y remanente, para realizar modelado.

La inclinación y declinación magnética del campo magnético total se tomaron como $90^{\circ}$ (polo norte magnético) y $0^{\circ}$, respectivamente.

Parámetros de entrada:

Campo magnético terrestre $=25177 \mathrm{nT}$

Inclinación magnética $=90^{\circ}$

Declinación magnética $=0^{\circ}$ 


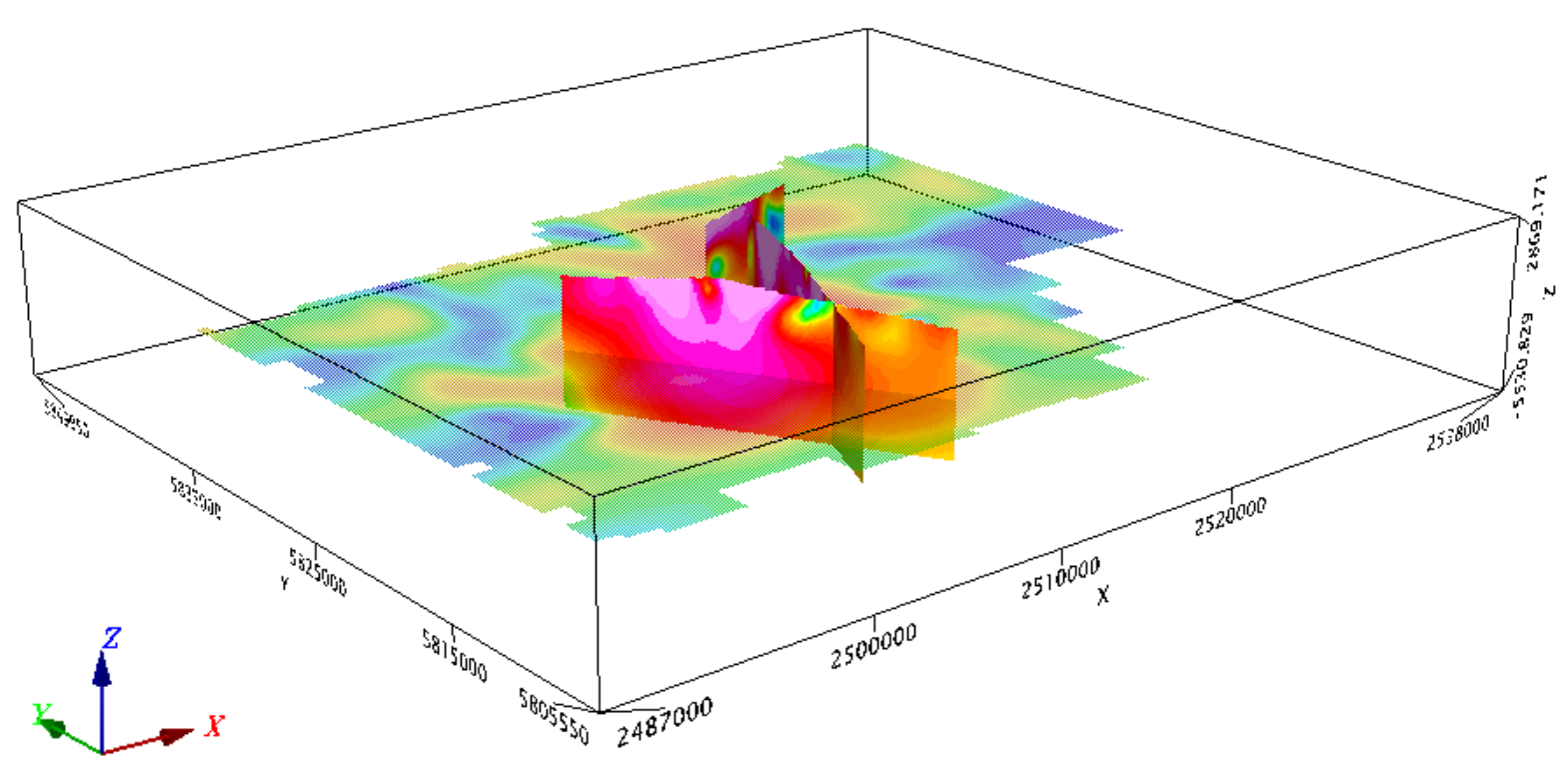

Figura 7.10: Perfiles para realizar modelado sobre el mapa de anomalías reducido al polo con los efectos de magnetización inducida y remanente.
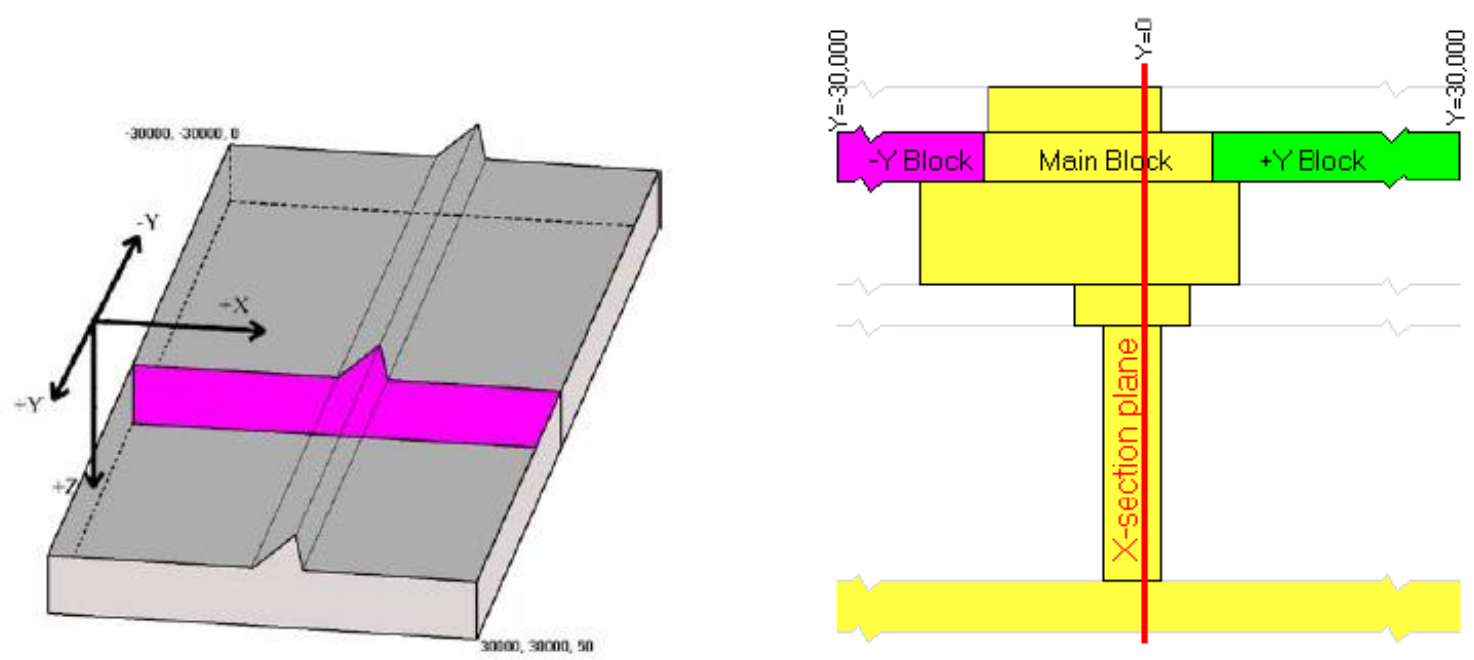

Figura 7.11: Convención de signos para modelado. Gráfico extraído de Geosoft.

Perfil AB: Orientación NO-SE. Este perfil modela las anomalías Rtp2 y Rtp3 y debe tener en cuenta el efecto de la fuente de las anomalías laterales Rtp1 (al oeste); Rtp4 y Rtp5 (al este). 

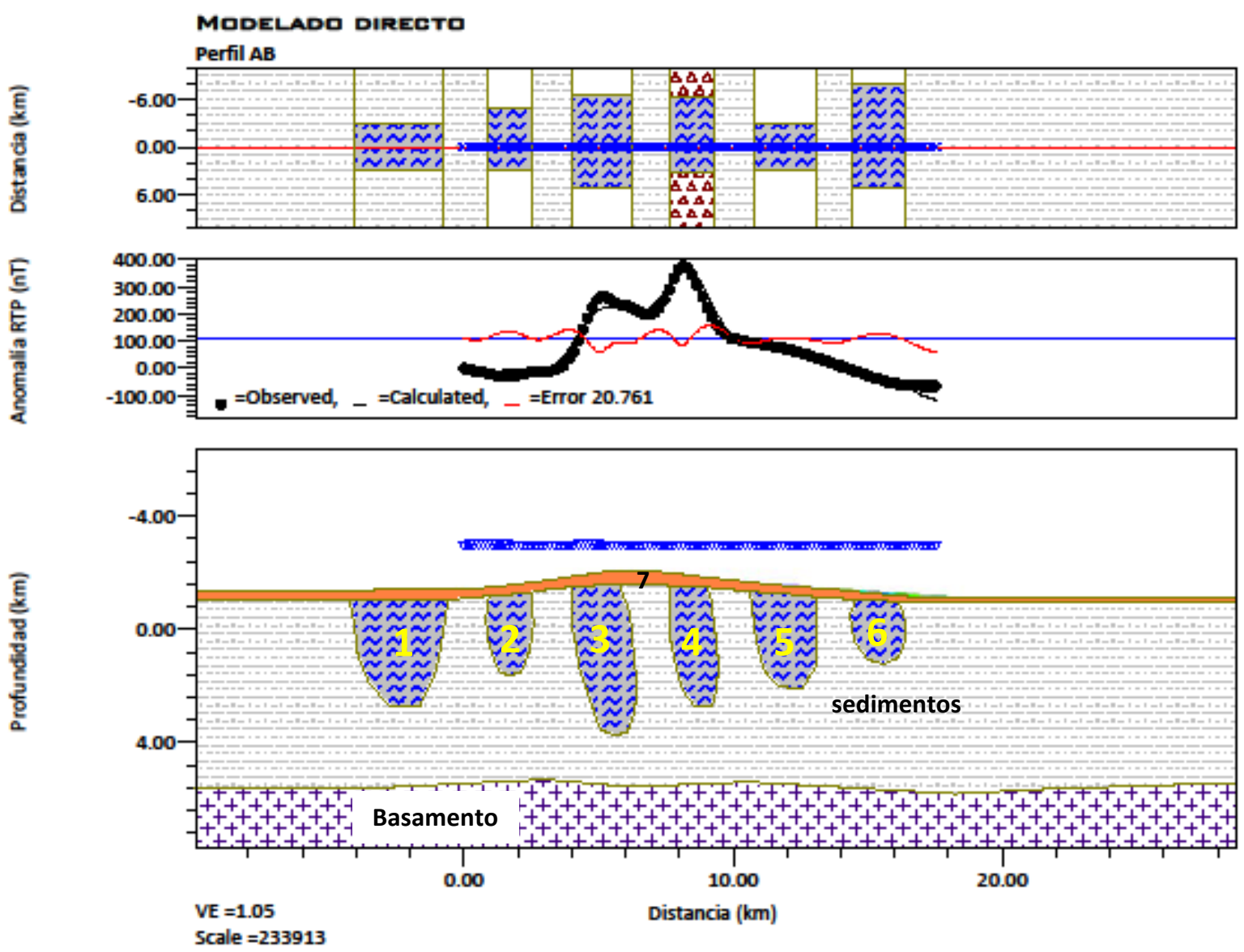

Figura 7.12. Modelo 2.75D sobre perfil AB representado en el mapa de la figura 7.9. De arriba hacia abajo: vista en planta de los cuerpos, cuya extensión lateral está expresada en $\mathbf{k m}$. Anomalía observada, calculada y curva de error (rojo). Modelado tomando en cuenta el modelo de inversión 3D (imagen superpuesta a los cuerpos) de la figura 7.10. A diferencia del modelado 3D, la dirección de $z$ es positiva debajo del nivel medio del mar.

Tabla 7.1. Parámetros utilizados en el modelo del perfil AB.

\begin{tabular}{|cccccc|}
\hline CUERPOS & NOMBRE & $M(\mathrm{~A} / \mathrm{m})$ & Susc $(\mathrm{SI})$ & $+\mathrm{Y}(\mathrm{km})$ & $-\mathrm{Y}(\mathrm{km})$ \\
\hline Basaltos sup & 7 & 2 & 0.03 & 33 & 37 \\
Rtp2 & 4 & 2.1 & 0.02 & 3.2 & 6.2 \\
Rtp3 & 3 & 0.8 & 0.02 & 5.2 & 6.4 \\
Igneo 1 & 2 & 0.9 & 0.0002 & 3 & 5 \\
Igneo 2 & 5 & 1.1 & 0.001 & 3 & 3 \\
Igneo 3 & 6 & 1 & 0.001 & 5 & 8 \\
Igneo 4 & 1 & 1.1 & 0.002 & 3 & 3 \\
Sedimentos & Sedimentos & 0 & 0.0 & 500 & 500 \\
Basamento & Basamento & 0.5 & 0.004 & 700 & 700 \\
\hline
\end{tabular}


Perfil CD: Orientación SO-NE. Este perfil modela las anomalías Rtp5, Rtp4 y Rtp7, el cual debe tener en cuenta el efecto de la fuente de las anomalías laterales Rtp3 y Rtp2 (al oeste).
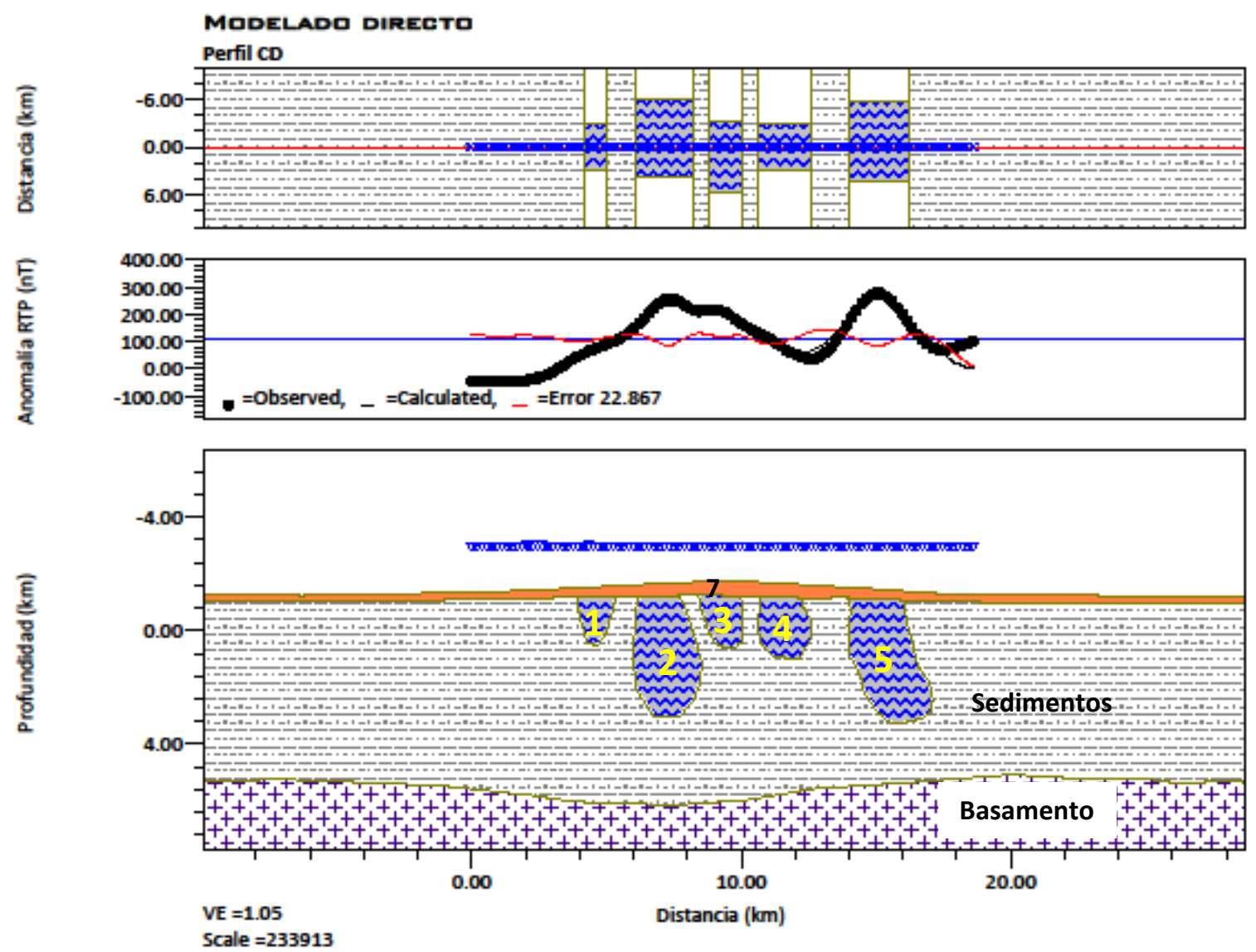

Figura 7.13. Modelo 2.75D sobre perfil CD representado en el mapa de la figura 7.9.

Tabla 7.2. Parámetros utilizados en el modelo del perfil CD.

\begin{tabular}{|cccccc|}
\hline CUERPOS & NOMBRE & $M(\mathrm{~A} / \mathrm{m})$ & Susc $(\mathrm{SI})$ & $+\mathrm{Y}(\mathrm{km})$ & $-\mathrm{Y}(\mathrm{km})$ \\
\hline Basaltos sup & 7 & 2 & 0.03 & 33 & 37 \\
Rtp4 & 3 & 1.2 & 0.004 & 5.6 & 3.2 \\
Rtp5 & 2 & 0.9 & 0.009 & 3.7 & 6 \\
Rtp7 & 5 & 1.6 & 0.002 & 4.2 & 5.8 \\
Igneo1 & 1 & 0.7 & 0.0025 & 3 & 3 \\
Igneo2 & 4 & 0.2 & 0.0009 & 3 & 3 \\
Sedimentos & Sedimentos & 0 & 0.0 & 500 & 500 \\
Basamento & Basamento & 0.5 & 0.004 & 700 & 700 \\
\hline
\end{tabular}


Perfil EF: Orientación SO-NE. Este perfil modela la anomalía Rtp8 y no existen fuentes laterales a considerar.

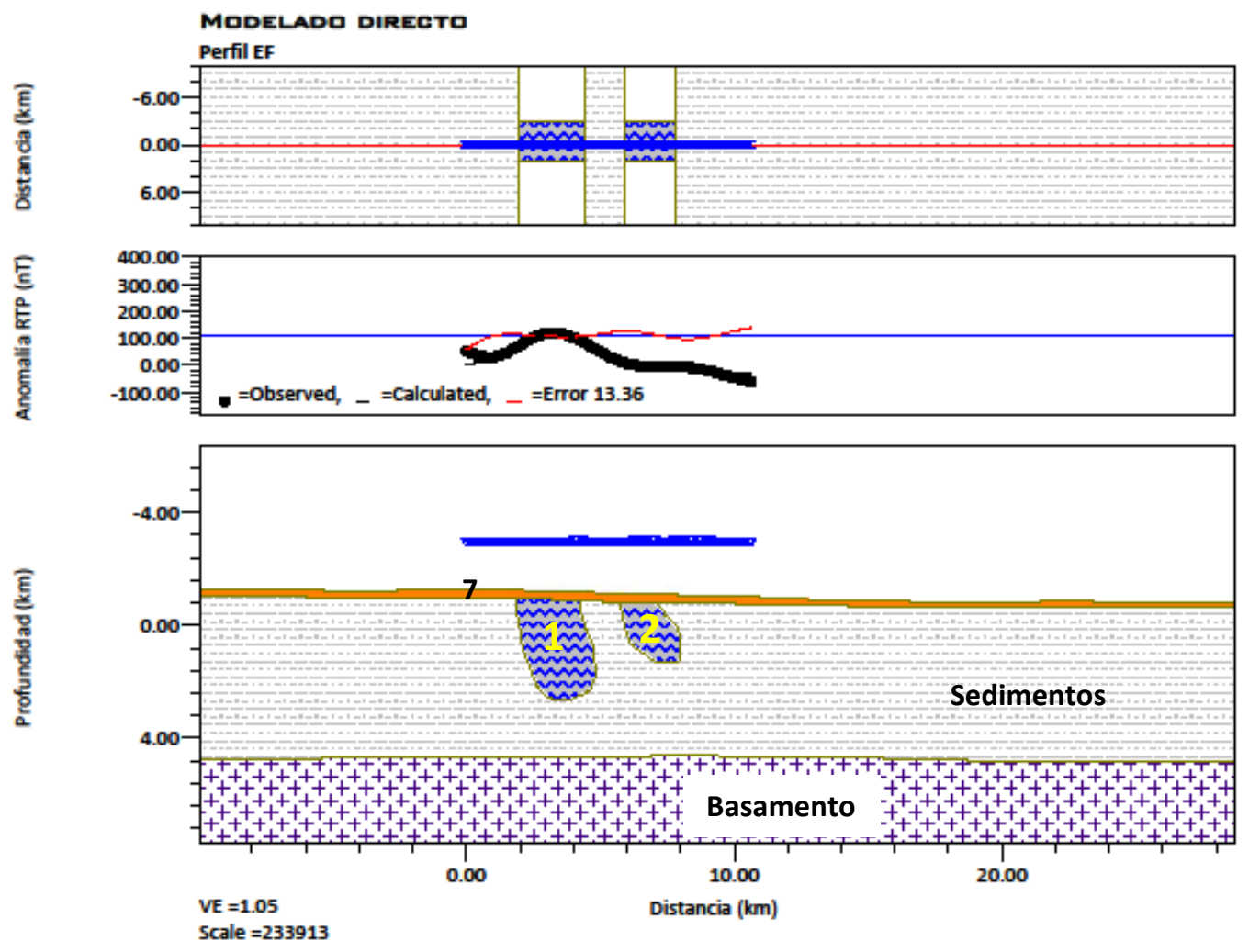

Figura 7.14. Modelo 2.75D sobre perfil EF representado en el mapa de la figura 7.9.

Tabla 7.3. Parámetros utilizados en el modelo del perfil EF.

\begin{tabular}{|cccccc|}
\hline CUERPOS & NOMBRE & $M(\mathrm{~A} / \mathrm{m})$ & Susc $(\mathrm{SI})$ & $+\mathrm{Y}(\mathrm{km})$ & $-\mathrm{Y}(\mathrm{km})$ \\
\hline Basaltos sup & 7 & 2 & 0.03 & 30 & 33 \\
Rtp8 & 1 & 1 & 0.003 & 2 & 3 \\
Igneo & 2 & 0.1 & 0.003 & 2 & 3 \\
Sedimentos & Sedimentos & 0 & 0.0 & 500 & 500 \\
Basamento & Basamento & 0.5 & 0.004 & 700 & 700 \\
\hline
\end{tabular}




\subsubsection{Modelado 2.75 D gravi-magnetométrico de los datos del VAM}

El objetivo de elaborar un modelo gravimétrico es obtener una imagen de la geometría y distribución de densidades del subsuelo, de modo que la respuesta gravimétrica se ajuste a las anomalías observadas. Debido a la no unicidad de los métodos potenciales, distintos modelos sobre un mismo perfil pueden dar la misma respuesta. Sin embargo, si se cuenta con información geológica y geofísica, se puede construir un modelo más confiable.

El modelo se construyó sobre la anomalía residual de Bouguer de la figura $6.11 \mathrm{~d}$ y la anomalía magnética sin reducir al polo de la figura 5.5 (Lince Klinger et al., 2014; Lince Klinger et al., 2015). Para ello se trazó un perfil con dirección NO-SE sobre una línea sísmica, el cual se extiende más allá de los límites de ésta (figura 7-15).

Se tomó la inclinación y declinación magnética que tiene en cuenta la magnetización por remanencia además de la de inducción.

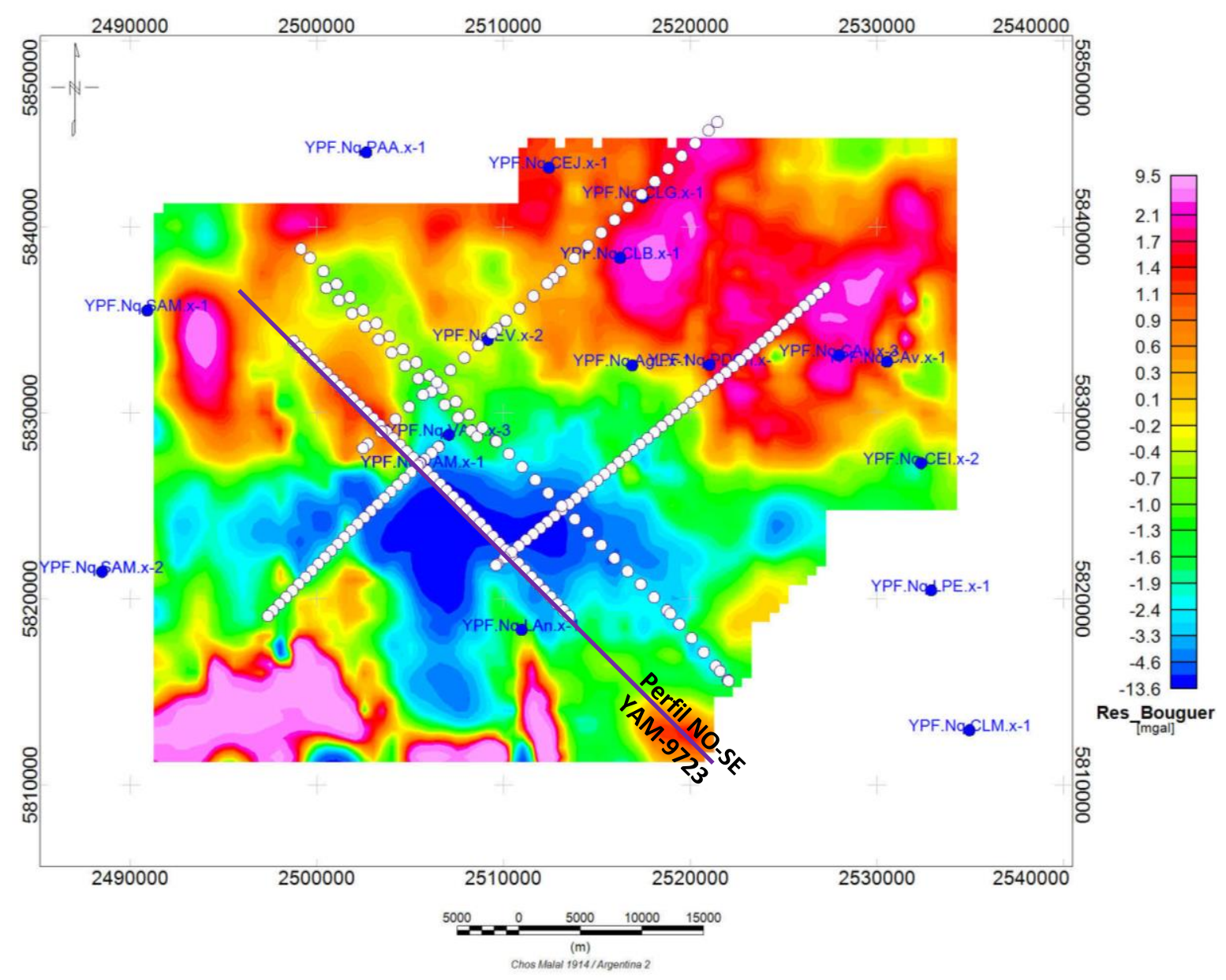

Figura 7.15 Sección NO-SE sobre línea sísmica YAM-9723 para modelado 2.75D (línea violeta). Los círculos blancos representan los puntos de explosión de las líneas sísmicas 2D reprocesadas en el área. El perfil gravi-magnetométrico es más extenso de lo que lo es la línea sísmica. 


\section{Parámetros de entrada:}

Campo magnético terrestre $=25177 \mathrm{nT}$

Inclinación magnética $=38^{\circ}$

Declinación magnética $=177^{\circ}$

La asignación de las densidades se realizó en base al promedio de las medidas en pozos cercanos. Se modelaron dos capas del periodo Terciario-Cuaternario, denominadas Basalto sup y Basalto central, una capa de sedimentos correspondiente al Cretácico (Gr Neuquén, Rayoso y Mulichinco) y otra del Jurásico (Quintuco, Vaca Muerta, Tordillo y Auquilco), y material intruido en el conducto central del volcán, Dique 1 al 6 (figura 7.15). Además, se tuvo en cuenta el basamento. Las densidades utilizadas en el modelo están representadas en la tabla 7.4. Las profundidades de estas capas se pusieron de acuerdo a la información de los pozos del área.

El mapa gravimétrico es más suave que el magnético, es decir tiene menor contenido de alta frecuencia comparado con el magnético, lo cual difculta el modelado conjunto como se observa en la figura 7.16. Por otro lado, no existe evidencia de correlación entre los datos gravimétricos y magnetométricos. El modelado gravimétrico sobre el perfil responde mejor que el magnético con un error de ajuste aceptable.

Tabla 7.4. Parámetros utilizados en el modelo del perfil gravi-magnetométrico.

\begin{tabular}{|ccccccc|}
\hline CUERPOS & NOMBRE & Den $\left(\mathrm{g} / \mathrm{cm}^{3}\right)$ & $M(\mathrm{~A} / \mathrm{m})$ & Susc $(\mathrm{SI})$ & $+\mathrm{Y}(\mathrm{km})$ & $-\mathrm{Y}(\mathrm{km})$ \\
\hline Basaltos sup & 7 & 2.75 & 2 & 0.03 & 33 & 37 \\
Basalto central & 8 & 2.35 & 2 & 0.03 & 15 & 15 \\
Dique 1 & 1 & 2.44 & 0.02 & 0.02 & 3 & 5 \\
Dique 2 & 2 & 2.45 & 0.1 & 0.006 & 3 & 5 \\
Dique 3 & 3 & 2.44 & 0.2 & 0.02 & 3 & 3 \\
Dique 4 & 4 & 2.39 & 0.01 & 0.0001 & 6 & 5 \\
Dique 5 & 5 & 2.41 & 0.8 & 0.008 & 1 & 8 \\
Dique 6 & 6 & 2.39 & 0.1 & 0.03 & 9 & 0.5 \\
Cretácico & Cretácico & 2.38 & 0 & 0 & 500 & 500 \\
Jurásico & Jurásico & 2.44 & 0 & 0 & 500 & 500 \\
Basamento & Basamento & 2.7 & 0.5 & 0.004 & 700 & 700 \\
\hline
\end{tabular}



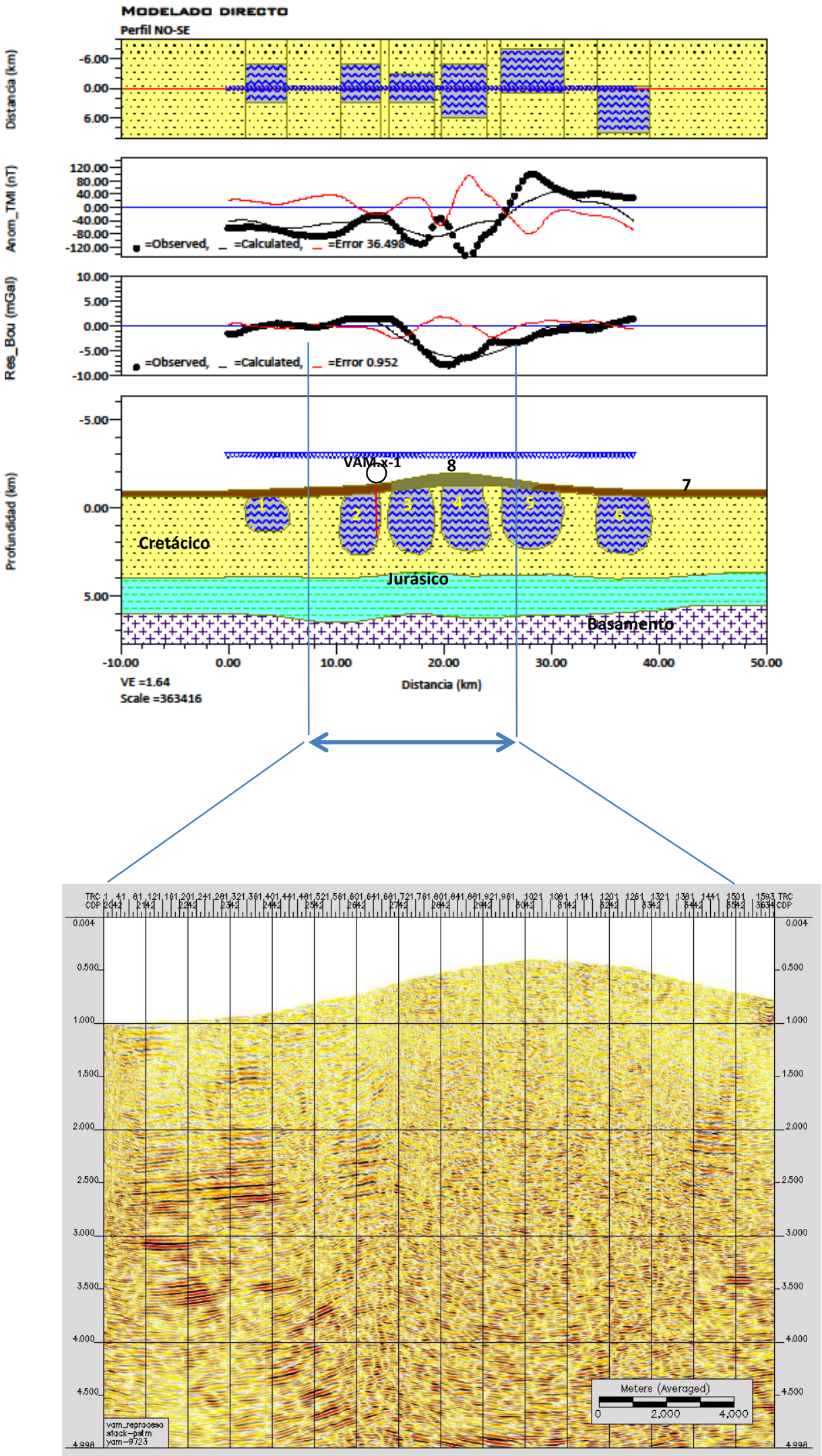

Figura 7.16 Modelo gravi-magnetométrico sobre perfil NO-SE de la figura 7.15. Proyección del pozo VAM.x-1 sobre el modelo. Cota del pozo: $1676 \mathrm{~m}$ sobre nivel medio del mar, profundidad final del pozo $3486 \mathrm{~m}$. Debajo: Imagen de la línea sísmica YAM-9723 sobre la cual se ralizó el modelo. 
Al cuerpo central debajo del volcán, Dique 1, se le asignó una densidad más baja que la registrada para los cuerpos intrusivos en los pozos del área, para poder explicar el mínimo gravimétrico observado en los datos. Esto se explica por la presencia de gran cantidad de fracturas y el posible colapso del conducto central, como se mencionó en el Capítulo VI. A otro cuerpo ígneo en la ladera del volcán, Dique 2, se le ha asignado una densidad de 2.7 $\mathrm{g} / \mathrm{cm}^{3}$.

Entre todos los parámetros geofísicos, la densidad de las rocas tiene un rango de variación pequeño. Los distintos flujos de lava que caracterizan al plateau tienen composiciones similares entre sí y a la de los cuerpos ígneos intrusivos, como se observa en las rocas provenientes de perforaciones. Las rocas piroclásticas, pómez y otras con densidades intrínsicamente bajas, constituyen un porcentaje muy pequeño del volumen total de los productos del volcán. Esto se traduce en la dificultad para discriminar unidades y estructuras dentro del complejo volcánico con gravimetría.

\subsection{Resultados}

- $\quad$ La máxima profundidad alcanzada por los cuerpos en los modelos magnéticos es 3.8 $\mathrm{km}$ bnm. Estos cuerpos aparecen agrupados en la zona central con una configuración pseudo-radial y elongados principalmente en la dirección NO-SE. Esta última orientación podría indicar que las estructuras intrusivas someras del volcán están controladas por las fallas de Entre Lomas (períodos Jurásico tardío y Cretácico Superior) de dirección NO-SE, las cuales afectan el basamento debajo del sector oriental del edificio volcánico (Ventura et al., 2012; figura 5.12).

- Teniendo en cuenta estos resultados, los cuerpos modelados en los perfiles 2.75D, se concentran debajo del crater central con una extensión en profundidad inferior a los 4 $\mathrm{km}$. Estos resultados son consistentes con los cuerpos obtenidos del modelado de inversión MVI (figura 5.16).

- La respuesta producida por el modelo gravi-magnetométrico se debe principalmente a cuerpos fuente ubicados en los primeros kilómetros de la corteza. 


\section{CAPÍTULO VIII}

\section{DISCUSIÓN GENERAL Y CONCLUSIONES}

En el presente capítulo se hace una síntesis de los resultados obtenidos a lo largo de este trabajo, integrando los resultados parciales más importantes que han sido desarrollados en los capítulos precedentes. Además, se discuten las distintas técnicas aplicadas en el análisis de los datos provenientes del área de estudio, que constituyen los pilares para el logro de los objetivos de esta tesis. Se muestra la eficacia de cada uno de los métodos y se ponen de manifiesto las limitaciones encontradas en este recorrido. Finalmente, se interpreta, desde el punto de vista geológico y volcanológico, el origen de las anomalías magnéticas y gravimétricas observadas en el volcán Auca Mahuida.

\subsection{Discusión sobre los resultados}

El hecho de trabajar en un área en la que se cuenta con información de subsuelo, condiciona y restringe los datos geofísicos y facilita su interpretación. Los pozos aportan información valiosa sobre la secuencia estratigráfica, sus características y geometría, la cual es útil para interpretar anomalías magnéticas y gravimétricas.

La estratigrafía local se puede subdividir en cuatro unidades principales: un basamento magnético, la secuencia sedimentaria no magnética, rocas magnéticas intrusivas y el plateau basáltico superficial magnético. Esta configuración permitió establecer una correlación entre las profundidades posibles de las fuentes magnéticas y gravimétricas y sus longitudes de onda locales y regionales.

Se pudo corroborar a partir de mediciones provenientes de perforaciones que el espesor de las coladas basálticas de superficie, alcanza aproximadamente $400 \mathrm{~m}$ en el conducto central del volcán y va disminuyendo hacia la periferia. Los cuerpos ígneos intercalados en la secuencia sedimentaria se alojan principalmente en las formaciones Quintuco-Vaca Muerta, Mulichinco, y el Grupo Cuyo. El espesor de estos cuerpos, comprobados en pozos exploratorios, varía entre los 70 y 140 metros. También se observa la presencia de rocas ígneas en otros niveles.

Desde el punto de vista magnético, la estimación de la profundidad de la isoterma 
Curie es crítica, ya que a profundidades mayores, no habrá contribución de fuentes magnéticas. Las mayores temperaturas registradas en el área a profundidades de aproximadamente $4 \mathrm{~km}$ bajo nivel del mar, son del orden de $180^{\circ} \mathrm{C}$, bastante menor que la temperatura de Curie para minerales como la magnetita, $580^{\circ} \mathrm{C}$ y hematita, $610^{\circ} \mathrm{C}$. Se puede asegurar que los cuerpos de roca ígnea intruidos en la secuencia sedimentaria en esta porción de la cuenca, no están térmicamente desmagnetizados, como se espera además, por la edad del volcán, y son los que contribuyen a las anomalías magnéticas del mismo.

Las propiedades magnéticas de las rocas - magnetización remanente natural (MRN) y susceptibilidad magnética- son parámetros que intervienen en la elaboración de modelos geológicos a partir de anomalías magnéticas. Es importante contar con datos medidos además de valores típicos encontrados en la literatura, porque permiten construir modelos más realistas. Sin embargo, es habitual que estas mediciones se realicen en la superficie y no provengan de sondeos, lo cual constituye una limitación para la interpretación de los resultados. Incluso los datos en profundidad pueden no ser representativos ya que el proceso de extracción puede alterar las propiedades magnéticas, además de no reflejar la variabilidad espacial de dichas propiedades en escalas mayores.

La determinación de la intensidad, dirección de inclinación y declinación de la magnetización de las muestras recolectadas en el campo, permitió hacer hipótesis concernientes a las características magnéticas de las intrusiones, en particular por la presencia de cuerpos con magnetización reversa debajo del cráter central del volcán. La mayor parte de ellas se extrajeron de la zona del edificio central, tienen magnetización remanente elevada y el $65 \%$ presenta polaridad reversa (tabla 4.1). Suponiendo que estas muestras tienen edades similares a las determinadas en dataciones radimétricas de estudios previos (Kay et al., 2006; Ramos and Folguera, 2010), se puede afirmar que el vulcanismo se desarrolló principalmente dentro del Cron Matuyama en el que predomina polaridad reversa (entre los subcrones de Olduvai y Jaramillo, figura 4.8).

Como se mencionó en el Capítulo II, se conocen al menos tres fases magmáticas: Oligoceno- Mioceno Inferior, Mioceno Medio -Plioceno y la más reciente Plioceno-Pleistoceno Inferior. Sólo se ha podido establecer la polaridad de las rocas del último ciclo (rocas intrusivas someras y superficiales del plateau) de edades inferiores a 3 M.a. Si se tiene en cuenta que las rocas extrusivas están genéticamente relacionadas con las intrusivas del volcán, es razonable suponer que ambas tendrán la misma dirección promedio de magnetización. Hasta el momento no se tiene información paleomagnética de rocas más antiguas.

La magnetización de las rocas volcánicas es mucho mayor que la de las rocas sedimentarias y metamórficas (consideradas no magnéticas), con lo cual en una zona volcánica, el contraste de magnetización entre estas últimas y los cuerpos ígneos da origen a anomalías cuya intensidad y signo son controlados por la litología.

El campo de anomalías magnéticas del volcán Auca Mahuida (figuras 5.1) muestra un conjunto de fuentes con una magnetización predominantemente reversa, situado alrededor del cráter central con longitudes de onda en el rango de 1.5 a $5 \mathrm{~km}$. 
En el mapa de anomalía magnética regional (figura 5.11 a) se observa que el área del VAM se sitúa entre una zona de valores bajos del campo magnético cortical (sector occidental R4) y una zona de bajo gradiente (sector oriental). Hacia el noreste y sureste se ubican dos lóbulos positivos de longitud de onda larga en correspondencia con el levantamiento de rocas del basamento debido a la acción del fallamiento directo de Entre Lomas (Cristallini et al., 2009). Estas observaciones fundamentan que las longitudes de onda corta del campo de anomalías del VAM sean atribuidas a fuentes volcánicas. Si la lava fuese la fuente de las anomalías del campo medido, la intensidad de ésta última sería mucho más alta. Sin embargo, como se analizó anteriormente a través del cálculo del efecto topográfico de una capa de 2 $\mathrm{A} / \mathrm{m}$, las anomalías magnéticas observadas en el relevamiento local no se correlacionan con la topografía. Esto implica que las fuentes (intrusiones) se encuentran debajo del manto de lava y por lo tanto son significativas desde el punto de vista interpretativo volcánicoestructural.

Como se describió en el Capítulo V, el filtro de reducción al polo cuando se usa sólo la dirección del campo magnético terrestre, no logra transformar la anomalía TMI en una anomalía unipolar positiva o negativa, por lo tanto, no es el más apropiado para modelar el campo magnético del volcán Auca Mahuida. En cambio, si usamos la dirección del campo magnético actual para la inducción y la dirección de magnetización para la remanencia, se obtiene la transformación de la figura 5.6 b. Esta transformación funciona mejor para producir respuestas unipolares positivas, lo cual es consistente con la interpretación visual y las mediciones de las rocas.

Teniendo en cuenta los resultados de la aplicación del filtro de RTP, los modelos magnéticos 2.75D se construyeron sobre perfiles tomados en el mapa de anomalías reducido al polo con los efectos combinados de inducción y remanencia. El ajuste de las anomalías (observada y calculada) es aceptable si consideramos valores de susceptibilidad magnética acordes con los resultados determinados en las muestras, y la geometría de los cuerpos condicionada por la inversión 3D.

Los valores más altos de $|\boldsymbol{M}| / H$ obtenidos de la inversión MVI son del orden de 0.02 SI. Estos valores corresponden a una magnetización de $0.8 \mathrm{~A} / \mathrm{m}$, tomando como campo de inducción el Campo Geomagnético de Referencia de la fecha del relevamiento (IGRF 2000). Los valores obtenidos de la inversión son muy bajos comparados con los medidos en las muestras de campo. La diferencia se puede explicar mediante tres factores. Por un lado, estas lavas presentan porciones de escoria en el tope y la base, lo cual reduce significativamente los valores de la magnetización del conjunto respecto a las porciones muestreadas (en su mayoría libre de escoria, tabla 4.1, Capítulo IV). Además, dado que el campo de anomalías es la suma resultante de los efectos de todas las fuentes, y puesto que la lava consiste en la superposición de capas, el efecto de la magnetización total es menor que el que se esperaría debido a la presencia de magnetizaciones con direcciones opuestas, las cuales se cancelarían entre ellas. Por último, la superficie del muestreo paleomagnético, el cual abarca sólo $170 \mathrm{~km}^{2}$ (3.6 \% de todo el plateau) en la parte central del volcán (figura 4.4), podría no estar representando a todas las unidades del área. Se puede argumentar entonces, que es muy 
probable que la magnetización media de los productos volcánicos aflorantes del Auca sea menor que la medida en las muestras (Paine, et al., 2015).

Una cuestión relevante en todo estudio de métodos potenciales es establecer una correlación entre las anomalías magnéticas y gravimétricas. En general, se esperaría que las rocas ígneas intruidas en la secuencia sedimentaria tengan densidad y magnetización mayores. Sin embargo, existen ciertos factores físicos y tectónicos que pueden modificar ese contraste. La temperatura, alteración mineralógica, compactación, fracturas, son algunos ejemplos. En el caso de estudio, altos valores de susceptibilidad y magnetización no se corresponden con altas densidades. Las diferencias de densidad son muy pequeñas comparadas con las diferencias de susceptibilidades, por lo tanto, las respuestas de dos rocas con diferentes valores de densidad son menos conspicuas que las de dos rocas de diferente susceptiblidad.

El mínimo gravimétrico en el cráter central corresponde a una zona profundamente fallada y fragmentada donde la densidad original de las rocas se ha modificado como consecuencia de la actividad volcánica (ver Capítulo VI). De este modo se ha favorecido la intrusión de diques, lacolitos y filones capa; lo cual está de acuerdo con la presencia de un alto número de intrusivos encontrados en la inversión de los datos magnéticos y los filones capa identificados en la periferia del cráter mediante interpretación sísmica.

Las anomalías magnéticas son más angostas que las gravimétricas, por lo que estas últimas se interfieren más. El resultado es que el mapa gravimétrico es más suave que el magnético, con menor contenido de alta frecuencia comparado con el magnético. La anomalía de Bouguer del relevamiento de alta resolución (figura 6.5) muestra un mínimo relativo en el cráter del volcán. Desde este punto de vista, los datos magnéticos son representativos de fuentes de menor tamaño (longitudes de onda de 1 a $5 \mathrm{~km}$ ) ubicadas debajo del cráter central formando una estructura intrusiva con forma de anillo. Los datos gravimétricos en el mismo sector, en cambio, indican una disminución general de la densidad en un volumen cortical más grande (longitud de onda de unos $15 \mathrm{~km}$ ).

Como se mencionó anteriormente (Capítulo III), los datos sísmicos 3D existentes corresponden a la porción norte del volcán y no abarcan la zona del cráter. La interpretación de los horizontes sísmicos, y filones capa intruidos en la secuencia sedimentaria y fallas, describen el patrón de emplazamiento del magma dentro del volcán. La signatura sísmica del tope de los filones, está caracterizada por una fuerte amplitud y relacionada a un alto contraste de la impedancia acústica entre su tope y la roca de caja. A veces se puede identificar, además del techo, la base y en general son concordantes con la estratigrafía (figrua 6.24). La anomalía en amplitud está controlada fundamentalmente por el espesor del filón, el efecto de interferencia y en menor grado por la litología de la roca de caja y fluidos (Comeron et al., 2002).

Las consideraciones hasta aquí expuestas permiten formular una serie de conclusiones respecto a cómo el análisis e integración de los datos y resultados obtenidos han conducido a un mayor entendimiento de la estructura interna del complejo volcánico Auca 
Mahuida. Esas conclusiones se exponen en la siguiente sección.

\subsection{Conclusiones generales}

La aplicación de los datos aeromagnéticos en el complejo volcánico AM permitió identificar y definir características estructurales, como fallas y fracturas, así como la distribución y extensión de sus productos. También permitió identificar cuerpos intrusivos o volcánicos que no afloran, asociados a los contrastes magnéticos con las rocas encajonantes. Los resultados más importantes se enumeran a continuación:

- La interpretación del campo de anomalías se enfocó principalmente en la caracterización de las fuentes mediante técnicas del modelado directo e inverso. El análisis de los resultados del modelado, junto con los datos complementarios disponibles, reveló que estas fuentes están probablemente relacionadas con cuerpos intrusivos asociados al sistema de alimentación del volcán el cual, a su vez, está controlado por el patrón estructural local y regional. Esa correlación también se evidencia en la orientación y geometría de las anomalías magnéticas reducidas al polo y por la distribución de los cuerpos fuentes obtenidos por el procedimiento de inversión; los cuales se alinean principalmente en la dirección de los principales rasgos estructurales.

- Como resultado del modelado se obtiene una estructura del sistema de conductos someros del volcán que se puede caracterizar como sigue. Un cuerpo intrusivo con forma de anillo debajo del cráter central, y una serie de otros cuerpos elongados en sentido NO-SE, seguramente controlados por fallas de Entre Lomas (períodos Jurásico tardío y Cretácico Superior) de la misma dirección a profundidades de hasta 3-4 km.

- Esta configuración de cuerpos ígneos con un patrón pseudo-radial tiene una clara correlación con la distribución de las principales anomalías magnéticas, como se mostró en detalle en el Capítulo V (cuerpos indicados con S1 a S10 en el mapa de señal analítica).

- La integración de los datos ha permitido ampliar la información sobre la estructura del sistema de alimentación del VAM y sobre las rocas ígneas intrusivas intercaladas en la secuencia sedimentaria. Estudios geomorfológicos y estructurales del campo volcánico (Ventura et al., 2012) sugieren que hubo una transición de vulcanismo monogénico a poligénico, ya que los depósitos piroclásticos de los conos están generalmente cubiertos por lavas provenientes del sector central. Esto sugiere una evolución del emplazamiento de cuerpos ígneos, que fueron inicialmente de tipo 
dique y posteriormente de tipo filón capa en un ambiente tectónico en el cual se incrementó la compresión. Algunos de ellos fueron observados en algunas coronas de pozos.

- En las secciones sísmicas en zonas aledañas a la cima, se ha podido interpretar algunos filones capa intercalados en los niveles de las Formaciones Quintuco-Vaca Muerta.

- Fallas preexistentes sirvieron de conducto para el ascenso de magma el cual alteró las propiedades de las rocas sedimentarias vecinas. La formación de fracturas debido al calentamiento y posterior enfriamiento de los intrusivos y las rocas sedimentarias contiguas aumentó localmente la porosidad y permeabilidad, dando lugar a diferentes calidades de roca en el reservorio de hidrocarburos. Además, el vulcanismo probablemente contribuyó a la generación del hidrocarburo en la roca madre por efectos térmicos (Pángaro, et al., 2004; Vottero et al., 2005).

- La presencia de poros y vesículas influyen en la disminución de la densidad de las rocas, pudiéndose explicar los valores mínimos relativos obtenidos en los datos gravimétricos. El cutting de algunos pozos ubicados en la zona de este mínimo, describen a las rocas ígneas como traquitas, las cuales son rocas intermedias con densidades menores a los basaltos.

Es importante conocer la ubicación y profundidad en la que se encuentran emplazados los cuerpos fuente porque contribuye a interpretar las anomalías desde el punto de vista geológico. Sin embargo, la dificultad de conseguir una localización confiable de las fuentes, se debe a que las anomalías de interés tendrían que estar separadas de otras creadas por fuentes cercanas, pero en la naturaleza esto en general no ocurre.

La ubicación de las fuentes magnéticas obtenidas con los distintos métodos y su correlación se resume en el siguiente cuadro:

Tabla 8.1. Correlación entre fuentes magnéticas obtenidas con los métodos de RTP con inducción y remanencia ( $\left.R T P_{-} i+r\right)$, señal analítica (SA), y las anomalías modeladas con GMSYS (2.75D).

\begin{tabular}{|c|c|c|}
\hline \multicolumn{3}{|c|}{ Correlación entre fuentes de anomalías } \\
\hline RTP_i+r & SA & 2.75D \\
\hline I & & \\
J & S1,S3 & Rtp1, Rtp2, Rtp3 \\
K & S2, S5 & Rtp4, Rtp5 \\
L & S7 & Rtp7 \\
M & & \\
\hline
\end{tabular}


donde RTP_i+r, es la reducción al polo del efecto combinado de inducción y remanencia del campo de anomalías magnéticas del VAM, SA es la señal analítica y 2.75D son las anomalías modeladas con el software GMSYS de Geosoft.

En el siguiente cuadro se representa el rango de profundidades de los cuerpos obtenidos con las técnicas estudiadas a lo largo esta tesis, y su comparación con las profundidades de intrusivos observadas en el pozo VAM.x-1 (figura 6.26).

Tabla 8.2. Profundidad de los cuerpos magnéticos obtenidos a partir de la aplicación de las técnicas utilizadas en este capítulo. La profundidad está dada en kilómetros respecto del nivel medio del mar (nmm), positivo hacia arriba y negativo debajo.

\begin{tabular}{|c|c|c|c|c|c|}
\hline \multicolumn{5}{|c|}{ Profundidades obtenidas en km (nmm) } \\
\hline Profundidades & Espec. Pot. Radial & Euler & $\mathrm{MVI}$ & $2.75 \mathrm{D}$ & pozo VAM.x-1 \\
\hline Mínimas & -0.8 & -2 & -0.5 & -1.5 & -0.3 \\
Máximas & 1.8 & 0.5 & 4 & 4 & 2 \\
\hline
\end{tabular}

La metodología llevada a cabo en el tratamiento de los datos aeromagnéticos y aerogravimétricos consistió en la aplicación de adecuadas técnicas de análisis para la caracterización y distribución de las fuentes magnéticas y gravimétricas. En términos generales se puede afirmar que los métodos de análisis de anomalías magnéticas y gravimétricas se han mostrado aptos para caracterizar la geometría del sistema de conductos someros del volcán en estudio, y para estimar la profundidad de cuerpos ígneos que, en zonas volcánicas, pueden formar parte de sistemas de reservorios de hidrocarburos. 


\section{APÉNDICE A}

\section{Descripción litológica de pozos del área de estudio que atraviesan cuerpos ígneos}

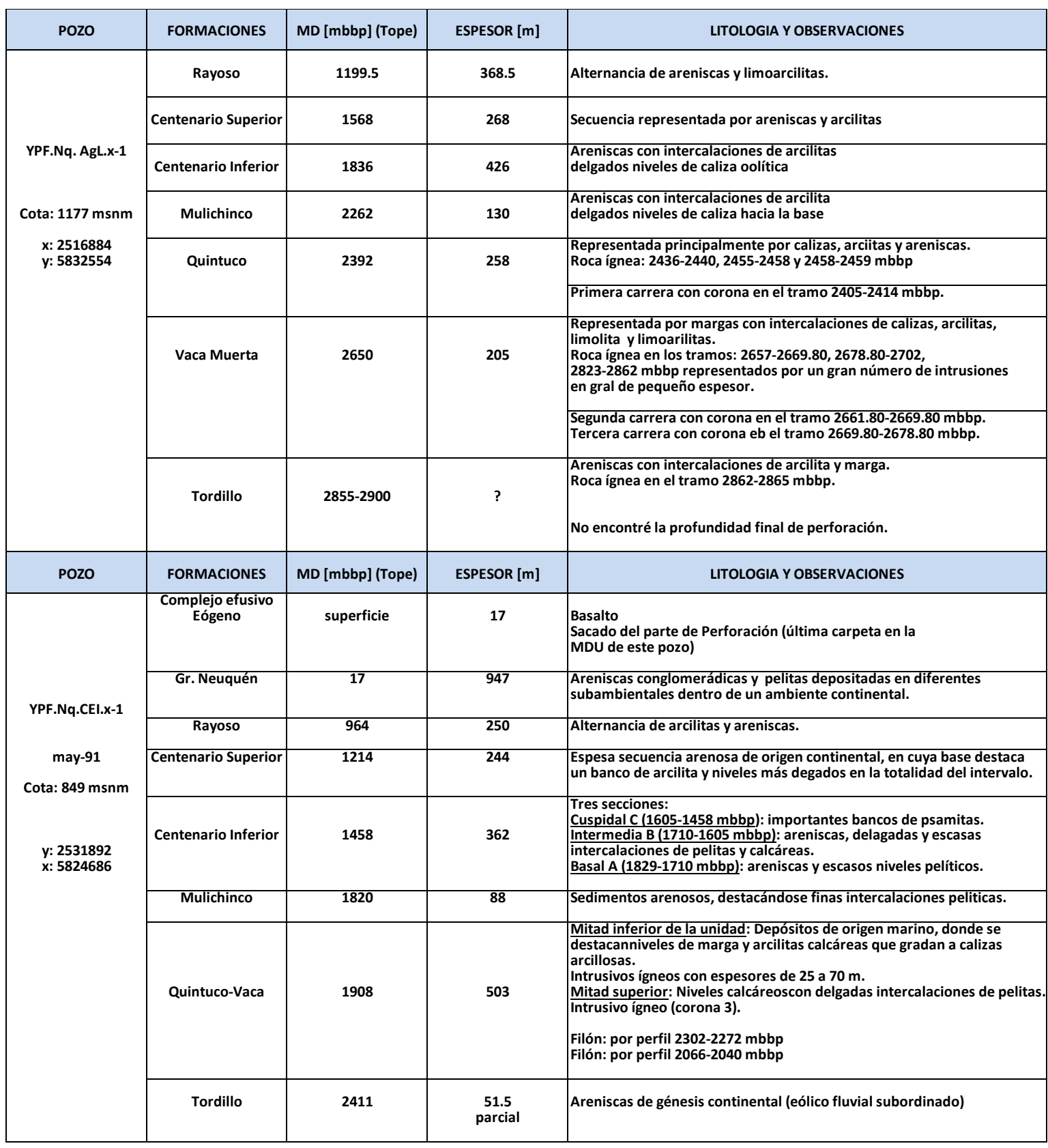




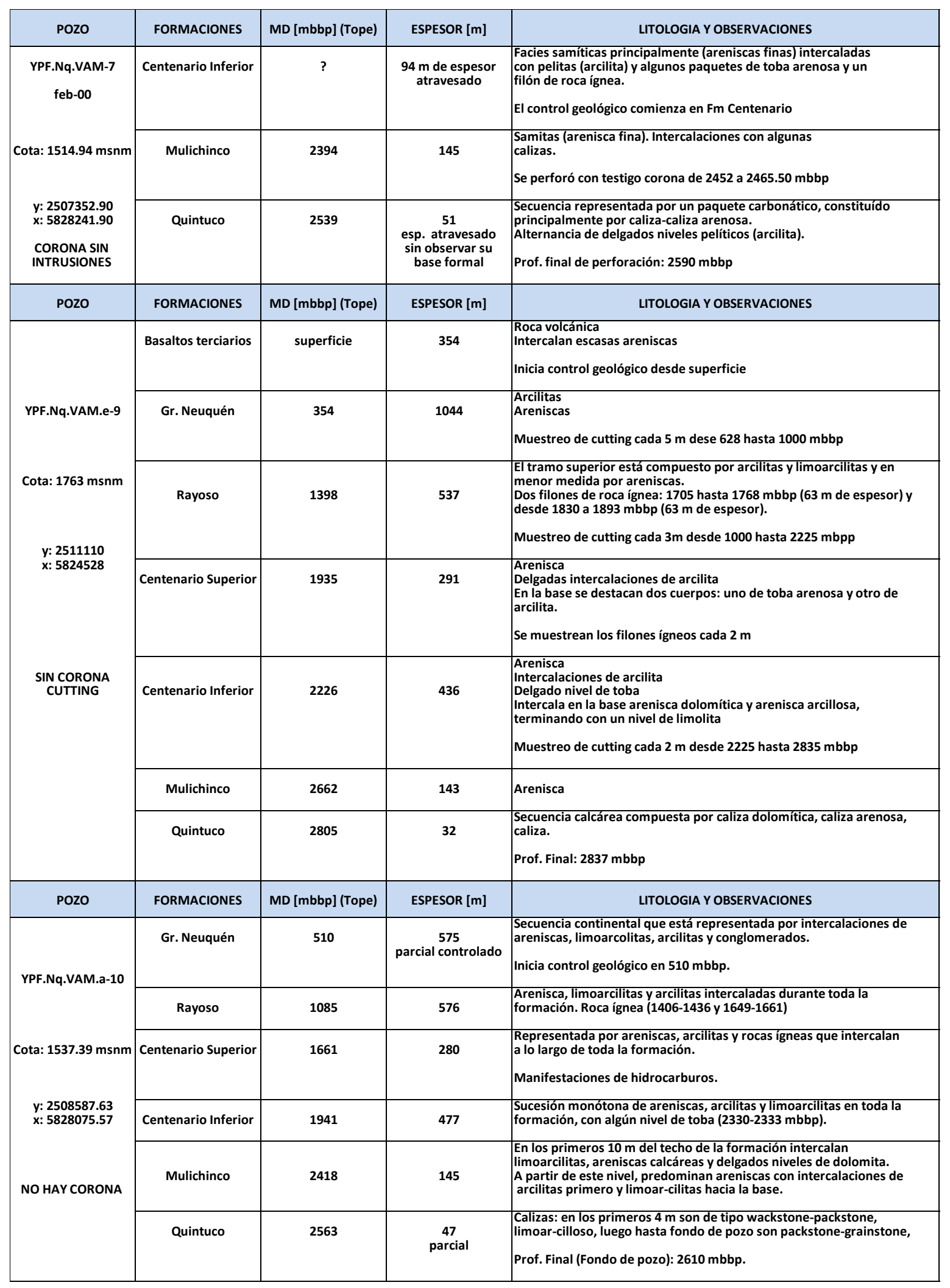




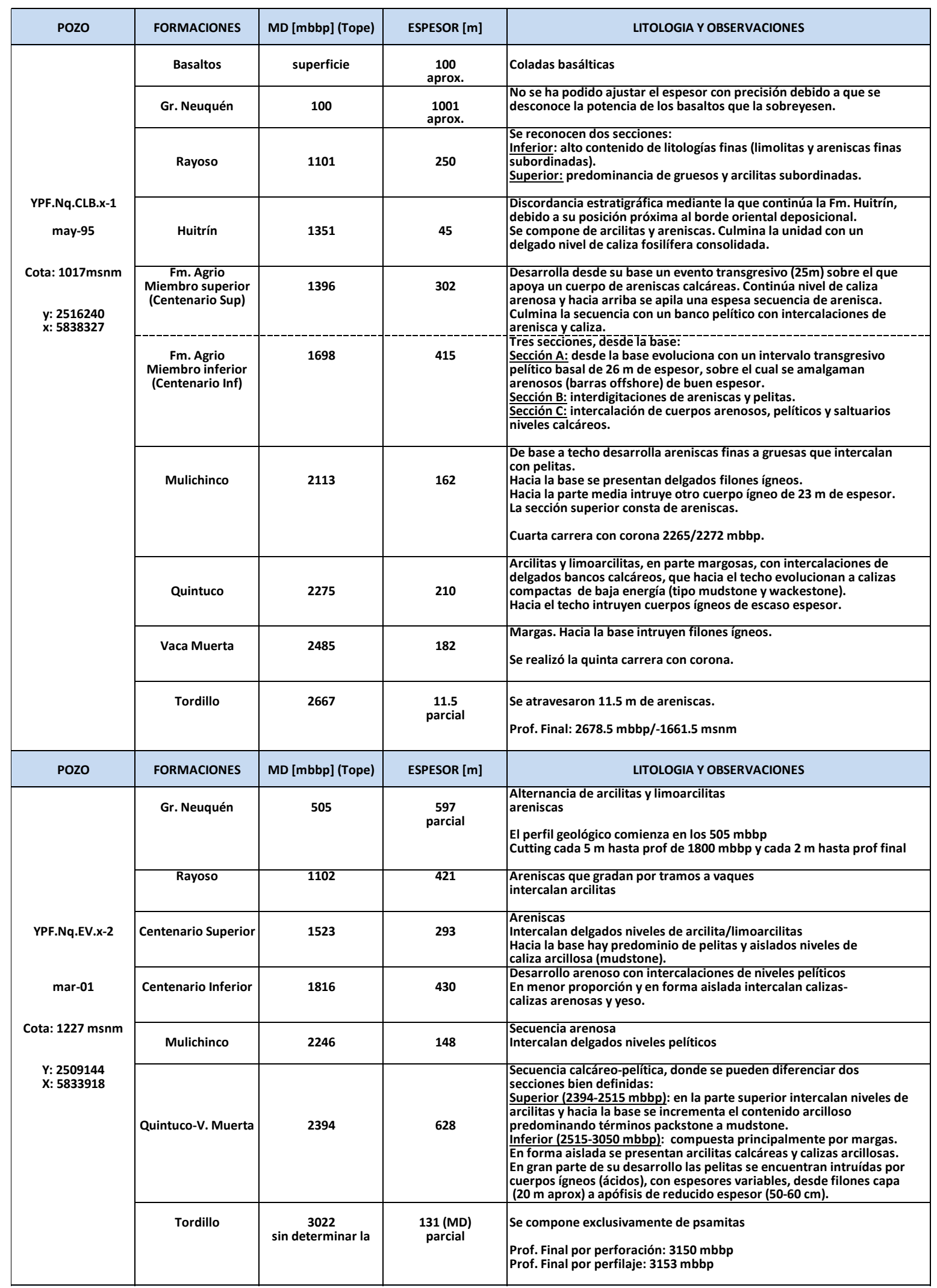




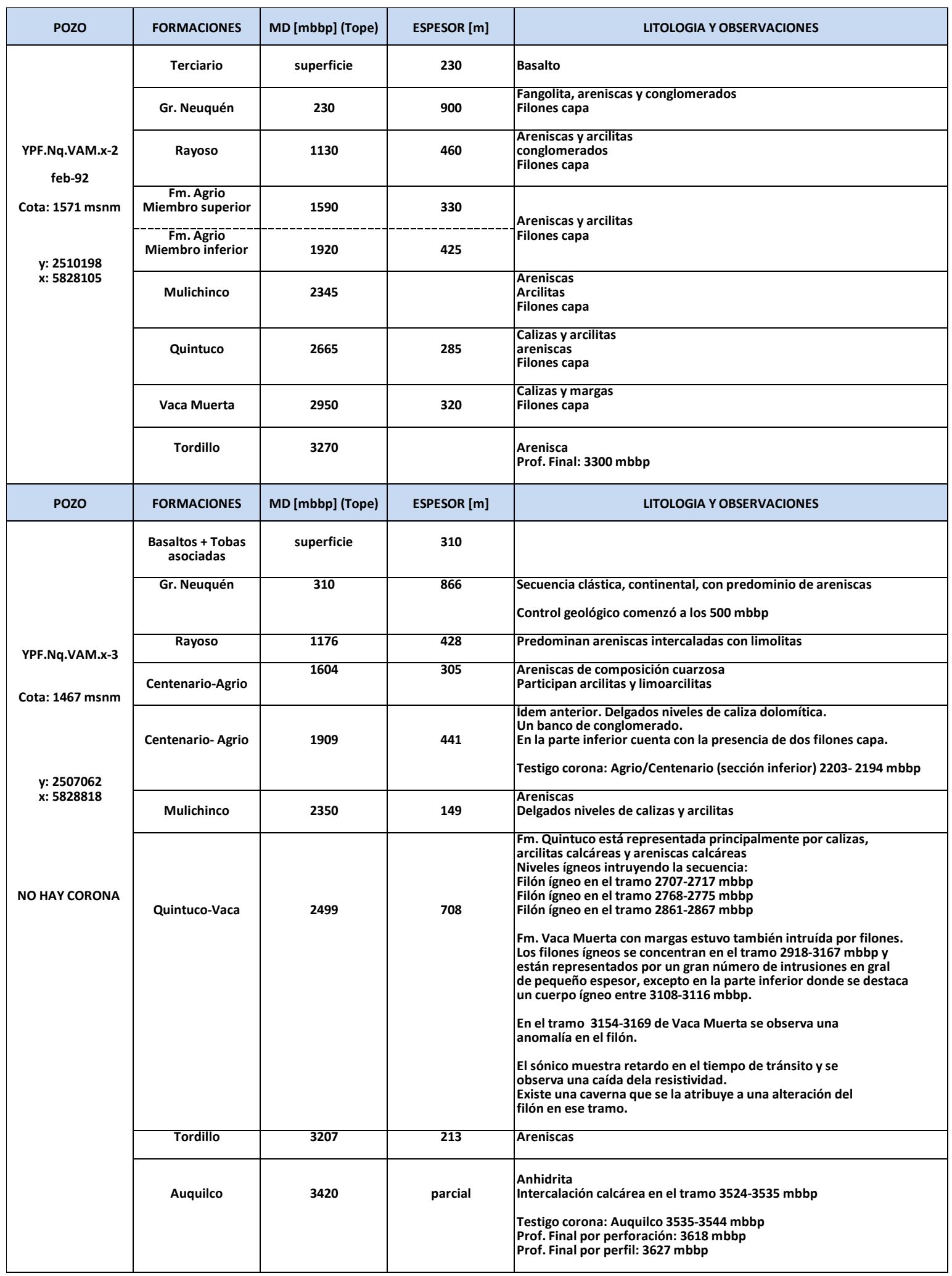




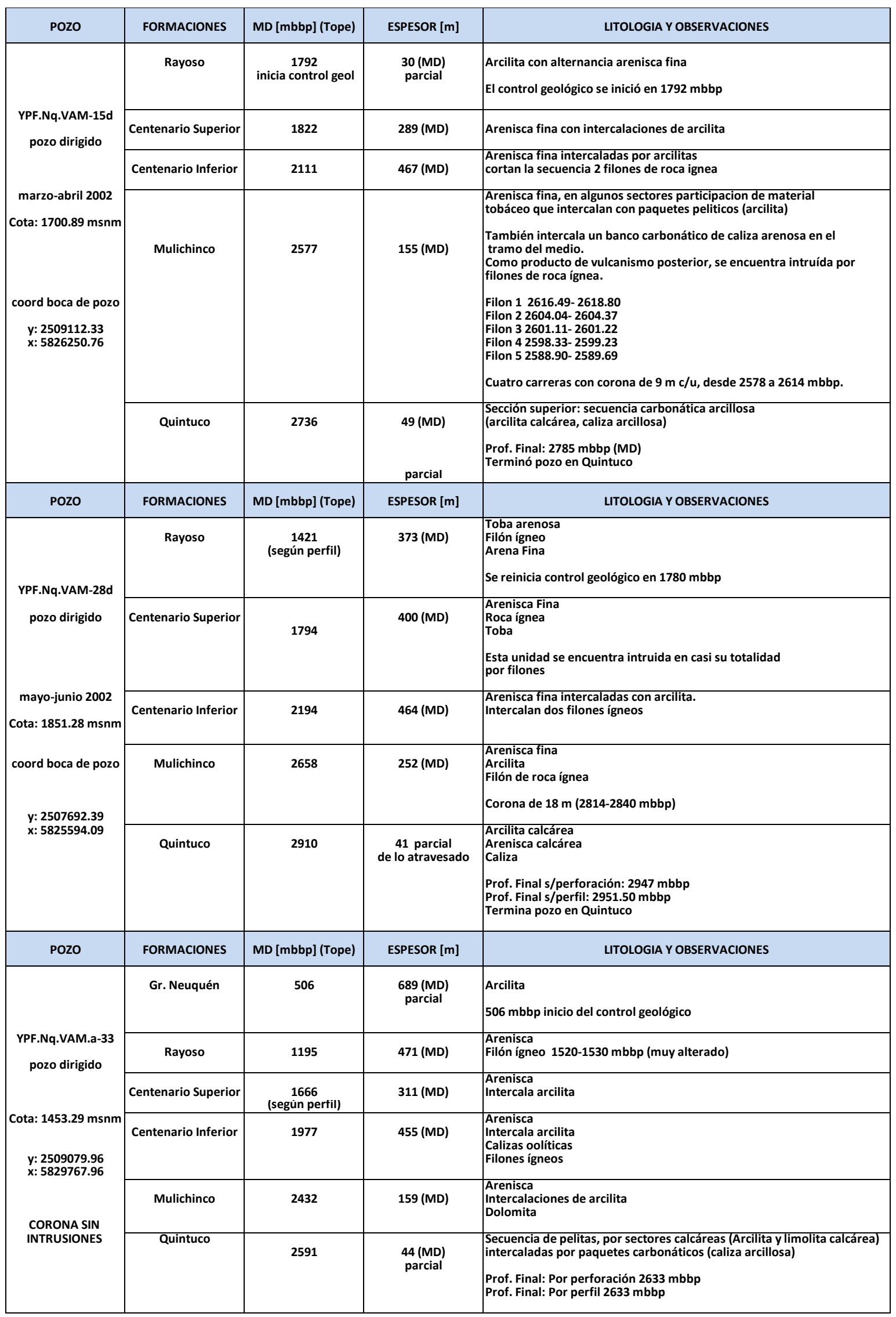




\begin{tabular}{|c|c|c|c|c|}
\hline \multirow[b]{2}{*}{ YPF.Nq.Lan.x-1 } & Cubierta basáltica & superficie & 214 & Basalto \\
\hline & Rayoso & 1251 & 129 & Secuencia continental, constituida por alternacia de arenisca \\
\hline $\begin{array}{l}y: 2510960 \\
x: 5818340\end{array}$ & Mulichinco & 2445 & 145 & $\begin{array}{l}\text { Facie predominantemente clástica de ambiente continental (fluvial). } \\
\text { En su tope se presenta un paquete arcilloso de amplia extensión } \\
\text { areal, que hacia el N y O aumenta su espesor y cambia de litología } \\
\text { para pasar a representar la base de Centenario. } \\
\text { Litología está compuesta por arenisca, con intercalaciones de dos } \\
\text { paquetes de } 50 \text { y } 70 \mathrm{~cm} \text { de arcilita con finos niveles arenosos. } \\
\text { Testigo corona en el intervalo } 2454-2463 \text { mbbp }\end{array}$ \\
\hline \multirow[t]{2}{*}{$\begin{array}{l}\text { CORONA SIN } \\
\text { INTRUSIONES }\end{array}$} & Quintuco-Vaca & 2590 & 669 & $\begin{array}{l}\text { Sección inferior (3259-3009 mbbp): pelítico-margosa que } \\
\text { comprende a la Fm. Vaca Muerta y el miembro inferior de la Fm. } \\
\text { Quintuco. } \\
\text { Sección superior: Representa al miembro medio y superior de dicha } \\
\text { formación, con interface transicional entre los mismos. En su base } \\
\text { hay pelitas y hacia su tope hay más energía despositándose calizas, } \\
\text { alternando con finos niveles de arcilita. Finaliza con bancos de } \\
\text { arenisca calcárea que gradan a caliza arenosa y fangolita. } \\
\text { Unidad atravesada por varios filones capa de pórfidos, de } \\
\text { composición variada. } \\
\text { Testigos corona en los intervalos } 2854-2863 \text { mbbp y } 2745-2754 \text { mbbp }\end{array}$ \\
\hline & Tordillo & 3259 & 211 & Areniscas \\
\hline \multirow[t]{2}{*}{ YPF.Nq.LPE.x-2 } & Rayoso + Centenario & 1065 & 994 & $\begin{array}{l}\text { La sección basal de } 84 \text { m presenta areniscas con intercalación de } \\
\text { de pelitas. El tope de esta sección lo define un banco de caliza. } \\
\text { Por encima se desarrolla una alternancia de areniscas con delgados } \\
\text { niveles de limoarcilita. } \\
\text { El límite superior de la unidad, lo marca la presencia de un cuello } \\
\text { pelítico-limoso de buen desarrollo areal, con el cual se inicia la } \\
\text { sedimentación del Grupo Neuquén. } \\
\text { El tramo arcilloso se halla desviculado de las sedimentitas } \\
\text { del Ciclo Andico por un evento erosivo (Discordancia } \\
\text { Intersenoniana). } \\
\text { El nivel arenoso presenta un marcado carácter continental, } \\
\text { no pudiendo diferenciarse de la unidad subyacente } \\
\text { (Fm. Centenario) en la presente posición de la cuenca. } \\
\text { Razón por la cual se las estudia en conjunto. }\end{array}$ \\
\hline & Quintuco-Vaca & 2059 & 492 & $\begin{array}{l}\text { Cuatro secciones (desde la base): } \\
\text { 1- Basal: margas, intercalándose con delgados niveles de caliza de } \\
\text { variable contenido arcilloso. En la porción media se atravesó una } \\
\text { roca ígnea filoniana de aprox. } 10 \mathrm{~m} \text { de espesor. } \\
\text { 2- Secuencia sedimentaria de limoarcilitas intercalándose hacia el } \\
\text { techo bancos de caliza. Cuerpo carbonático de } 25 \mathrm{~m} \text { de espesor en } \\
\text { el tope de la sección. } \\
\text { 3- En la porción basal presenta limoarcilitas, con escasas } \\
\text { intercalaciones de calizas arcillosas. Hacia el techo evolucionan } \\
\text { gradualmente a calizas limoarenosas. } \\
4-\text { La sección superior está representada por bancos de caliza } \\
\text { intercalándose en escasa proporción samitas calcáreas y arcilitas. } \\
\text { Tramos con corona Fm. Quintuco- Vaca Muerta } \\
2120-2126 \text { mbbp (corregido por perfil } 2124-2130 \mathrm{mbbp} \text { ) } \\
2127-2134.5 \mathrm{mbbp} \text { (corregido por perfil } 2131-2138.5 \mathrm{mbbp} \text { ) } \\
2441-2450 \text { mbbp (Corregido por perfil } 2445.5-2454.5 \mathrm{mbbp} \text { ). } \\
\text { Roca ígnea intrusiva. }\end{array}$ \\
\hline
\end{tabular}




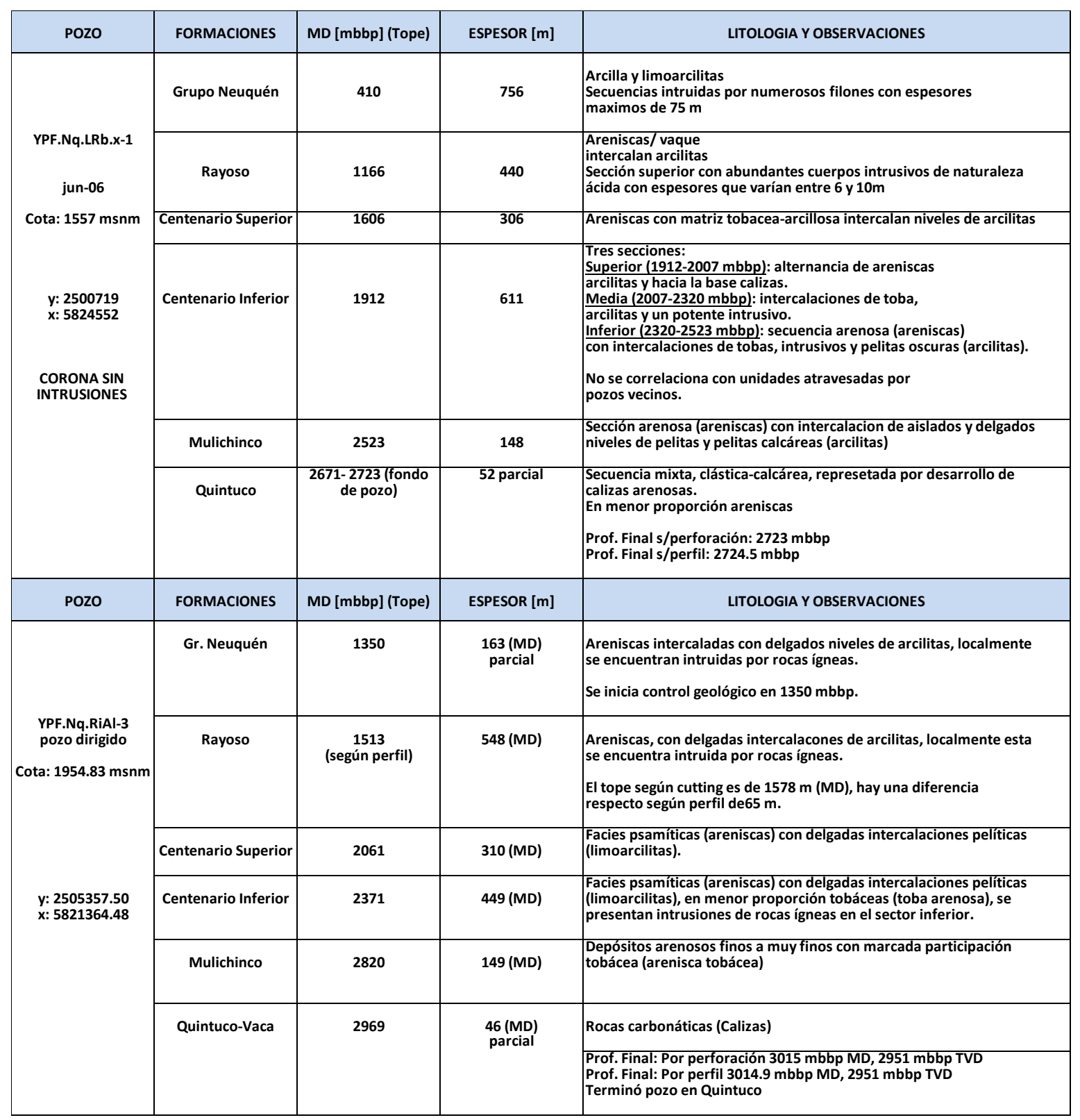




\begin{tabular}{|c|c|c|c|c|}
\hline POzO & FORMACIONES & MD [mbbp] (Tope) & ESPESOR $[\mathrm{m}]$ & LITOLOGIA Y OBSERVACIONES \\
\hline & $\begin{array}{l}\text { Terciario + } \\
\text { Gr. Neuquén }\end{array}$ & superficie & 1100 & $\begin{array}{l}\text { Está compuesto por areniscas con frecuentes intercalaciones de } \\
\text { fangolita. }\end{array}$ \\
\hline $\begin{array}{l}\text { YPF.Nq.SAM.x-1 } \\
\text { mar-80 } \\
\text { Cota: } 949 \mathrm{msnm}\end{array}$ & Rayoso & 1100 & 552 & $\begin{array}{l}\text { Se diferencian dos secciones: } \\
\text { 1- La basal de } 400 \mathrm{~m} \text { de espesor está constituída por arenisca con } \\
\text { intercalaciones de de limolita y arcilita. Presencia de delgados y } \\
\text { saltuarios niveles de yeso y caliza. } \\
\text { 2- La sección superior de } 152 \mathrm{~m} \text { de espesor es netamente pelítica } \\
\text { con alternancia de arcilita y limolita. } \\
\text { Tramos perforados con corona: } \\
\text { 1- Prof. } 2333 / 2342 \mathrm{mbbp} \text { (corregida } 2343 / 2352 \mathrm{mbbp} \text { ). } \\
\text { Fm. Centenario } \\
\text { 2-Prof. } 3308 / 3317 \mathrm{mbbp} \text { (corregida } 3317 / 3326 \mathrm{mbbp} \text { ). } \\
\text { Fm. Tordillo }\end{array}$ \\
\hline $\begin{array}{l}y: 2490820 \\
x: 5835545\end{array}$ & Centenario & 1652 & 788 & $\begin{array}{l}\text { Dos secciones representadas por sedimentos que sugieren } \\
\text { diferentes ambientes sedimentarios. } \\
\text { 1- La sección basal de } 188 \mathrm{~m} \text { de espesor está constituída por pelitas } \\
\text { y arenisca. } \\
\text { 2- La sección suprayacente de } 600 \mathrm{~m} \text { de espesor está formada por } \\
\text { sedimentitas (arenisca), e intercalaciones de arcilita y limolita y } \\
\text { saltuarios niveles de caliza y yeso. }\end{array}$ \\
\hline CUTTING & Mulichinco & 2440 & 165 & Constituída por arenisca con intercalaciones de arcilita y limolita. \\
\hline \multirow[t]{2}{*}{$\begin{array}{l}\text { CORONA SIN } \\
\text { INTRUSIONES }\end{array}$} & Quintuco-Vaca & 2605 & 673 & $\begin{array}{l}\text { Facies pelíticas que la caracteriza en casi la totalidad de la cuenca. } \\
\text { Potente secuencia de marga y arcilita que denota un ambiente de } \\
\text { deposición marino distal. } \\
\text { En la sección superior estas pelitas alternan con finos niveles de } \\
\text { limolita, caliza arcillosa y areniscas muy finas. } \\
\text { Cercano a la base se atravesó un filón capa de } 25 \text { mde espesor. }\end{array}$ \\
\hline & Tordillo & 3278 & 180 & $\begin{array}{l}\text { Arenisca (vaques). Se intercalan saltuarios y delgados niveles } \\
\text { tobáceos en el tercio inferior de la formación. } \\
\text { Testigo corona: } 3309 / 3326 \mathrm{mbbp}\end{array}$ \\
\hline
\end{tabular}




\section{APÉNDICE B}

Cálculo de una anomalía magnética en una placa de espesor variable. Blakely, 1981

Sea $\vec{v}$ un vector tri-dimensional denotado por:

$$
\vec{v}=\left(v_{x}, v_{y}, v_{z}\right)
$$

y la posición horizontal se escribe como $\vec{a}=(x, y)$. Consideremos un medio magnético (figura B1) confinado entre dos superficies $z_{1}(\vec{a})$ y $z_{2}(\vec{a})$. La dirección de la magnetización es constante y la intensidad varía solo en la dirección horizontal, esto es:

$$
m(x, y, z)=m(\vec{a}) \widehat{m}
$$

donde $m(\vec{a})$ es la intensidad y $\widehat{m}$ es el vector unitario en la dirección de magnetización. La anomalía $h(\overrightarrow{a)}$ es observada sobre un plano horizontal $z=0$ y es medido en la dirección de $\hat{h}$. Para la mayoría del campo total de anomalías en aplicaciones geofísicas, $\hat{h}$ puede asumirse paralelo al campo regional. Esta suposición no es válida si la magnitud de la anomalía es una fracción apreciable del campo magnético total.

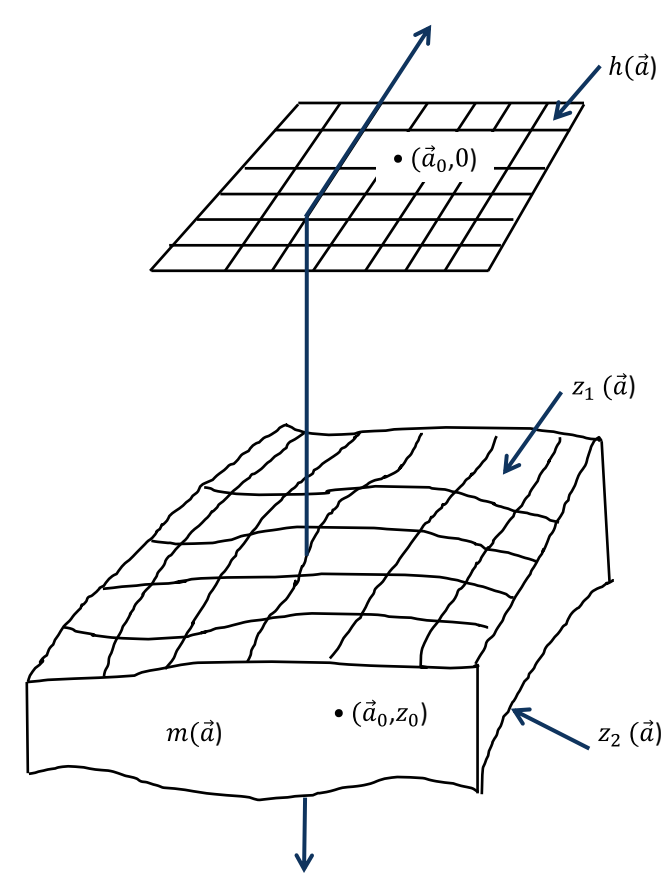

Figura B1. Geometría de la capa magnética.

La anomalía causada por un elemento simple de un cuerpo ubicado en $\left(\vec{a}_{0,}, z_{0}\right)$ es: 


$$
d h(\vec{a})=m\left(\vec{a}_{0}\right) t_{d}\left(\vec{a}-\vec{a}_{0}, z_{0}\right) d x_{0} d y_{0} d z_{0}
$$

donde $t_{d}\left(\vec{a}_{0,} z_{0}\right)$ es la anomalía de un dipolo simple. Integrando sobre los límites del cuerpo se tiene la anomalía causada por el cuerpo entero, dada por:

$$
h(\vec{a})=\int_{z_{1}(\vec{a})}^{z_{2}(\vec{a})} \iint m\left(\vec{a}_{0}\right) t_{d}\left(\vec{a}-\vec{a}_{0}, z_{0}\right) d x_{0} d y_{0} d z_{0}
$$

La transformada de Fourier de una función $f$ se define como:

$$
\mathcal{F}[f(\vec{a})]=\iint f(\vec{a}) e^{-i \vec{k} \cdot \vec{a}} d x d y
$$

donde $\vec{k}=\left(k_{x}, k_{y}\right)$ son las coordenados del número de onda. Tomando la transforma de Fourier de ambos miembros de la ecuación (B1) y cambiando el orden de integración, se llega a la integral (B2):

$$
\begin{array}{r}
\mathcal{F}[h(\vec{a})]=\iint m\left(\vec{a}_{0}\right) \int_{z_{1}(\vec{a})}^{z_{2}(\vec{a})} \iint m(\vec{a}) t_{d}\left(\vec{a}-\vec{a}_{0}, z_{0}\right) d x_{0} d y_{0} d z_{0} \\
=\iint m\left(\vec{a}_{0}\right) \int_{z_{1}(\vec{a})}^{z_{2}(\vec{a})} e^{-i \vec{k} \cdot \vec{a}} \mathcal{F}\left[t_{d}\left(\vec{a}, z_{0}\right)\right] d z_{0} d x_{0} d y_{0}
\end{array}
$$

La transformada de Fourier en el miembro derecho de la ecuación (B2) es la transformada de la anomalía al plano $z=0$ causada por un dipolo simple en $z=z_{0}$ y se escribe como:

$$
\mathcal{F}\left[t_{d}\left(\vec{a}, z_{0}\right)\right]=-2 \pi \phi(\vec{k})|\vec{k}| e^{-|\vec{k}| z_{0}}
$$

donde $\phi(\vec{k})$ es una función de los cosenos directores de $\widehat{m}$ y $\widehat{h}$. Sustituyendo la ecuación (B3) en la (B2), la integral sobre $z_{0}$ viene dada por:

$$
\mathcal{F}[h(\vec{a})]=2 \pi \phi(\vec{k}) \iint m\left(\vec{a}_{0}\right)\left\{e^{-|\vec{k}| z_{2}\left(\vec{a}_{0}\right)}-e^{-|\vec{k}| z_{1}\left(\vec{a}_{0}\right)}\right\} e^{-i \vec{k} \cdot \vec{a}} d x_{0} d y_{0}
$$

La integral doble que queda es una transformada de Fourier bidimensional, de modo que la ecuación (B4) se puede reescribir como:

$$
\mathcal{F}[h(\vec{a})]=2 \pi \phi(\vec{k}) \mathcal{F}\left[m\left(\vec{a}_{0}\right)\left\{e^{-|\vec{k}| z_{2}\left(\vec{a}_{0}\right)}-e^{-|\vec{k}| z_{1}\left(\vec{a}_{0}\right)}\right\}\right]
$$

Las dos exponenciales se pueden reemplazar por sus respectivos desarrollos en serie, 
cuya rapidez de convergencia depende de la elevación del origen. La elevación óptima es la del punto medio entre el máximo y el mínimo valor de $z_{1}(\vec{a})$ y $z_{2}(\vec{a})$. Si $\delta_{1}$ y $\delta_{2}$ son los valores de las medianas de $z_{1}(\vec{a})$ y $z_{2}(\vec{a})$ z1(a), respectivamente, entonces la ecuación (B5) se puede reescribir en la forma:

$$
\mathcal{F}[h(\vec{a})]=2 \pi \phi(\vec{k})\left\{e^{-|\vec{k}| \delta_{2}} \mathcal{F}\left[m\left(\vec{a}_{0}\right) e^{-|\vec{k}|\left(z_{2}\left(\vec{a}_{0}\right)-\delta_{2}\right)}\right]-e^{-|\vec{k}| \delta_{1}} \mathcal{F}\left[m\left(\vec{a}_{0}\right) e^{-|\vec{k}|\left(z_{1}\left(\vec{a}_{0}\right)-\delta_{1}\right)}\right]\right\}
$$

Luego, reemplazando las dos exponenciales internas por series de potencias y hacienda algunas simplificaciones menores, queda la ecuación siguiente:

$$
\mathcal{F}[h(\vec{a})]=2 \pi \phi(\vec{k}) \sum_{n=0}^{\infty} S_{n}(\vec{k})
$$

donde

$$
\begin{aligned}
& S_{n}(\vec{k})=\frac{-(|\vec{k}|)^{n}}{n !} \begin{cases}e^{-|\vec{k}| \delta_{2}} & \mathcal{F}\left[m\left(\vec{a}_{0}\right)\left(z_{2}\left(\vec{a}_{0}\right)-\delta_{2}\right)^{n}\right]\end{cases} \\
& \left.-e^{-|\vec{k}| \delta_{1}} \mathcal{F}\left[m\left(\vec{a}_{0}\right)\left(z_{1}\left(\vec{a}_{0}\right)-\delta_{1}\right)^{n}\right]\right\}
\end{aligned}
$$

Nótese que el término de $n=0$ en la ecuación (6) es:

$$
\mathcal{F}[h(\vec{a})]=2 \pi \phi(\vec{k})\left\{e^{-|\vec{k}| \delta_{2}}-e^{-|\vec{k}| \delta_{1}}\right\} \mathcal{F}\left[m\left(\vec{a}_{0}\right)\right]
$$

el cual es la transformada de Fourier de la anomalía sobre una placa horizontal con tope y base en $\delta_{1}$ y $\delta_{2}$, respectivamente. Los términos de orden superior representan las perturbaciones de la topografía sobre esta placa.

La ecuación (B6) muestra que la anomalía $h(\vec{a})$ sobre un cuerpo magnetizado puede obtenerse calculando la transformada inversa de Fourier de una suma ponderada de transformadas de Fourier de la magnetización $m(\vec{a})$, multiplicada por potencias de las superficies del tope $z_{1}(\vec{a})$ y la base $z_{2}(\vec{a})$. 


\section{APÉNDICE C}

\section{Problema directo 2D. Método de Talwani-Heirtzler (1964)}

La sección transversal de un cuerpo bidimensional de forma arbitraria se simplifica mediante un polígono irregular. Se calculan las expresiones de las anomalías magnéticas causadas por dichos cuerpos en sus componentes horizontal, vertical e intensidad total.

Consideremos la anomalía magnética causada por un prisma bidimensional semiinfinito KLMN como se observa en la figura C1. El prisma se extiende hasta infinito a lo largo del eje $x$ positivo e $y$ positivo. Calcularemos la anomalía causada por dicho prisma en el origen. Para ello primero consideraremos el efecto causado por una varilla de sección $A B C D$.

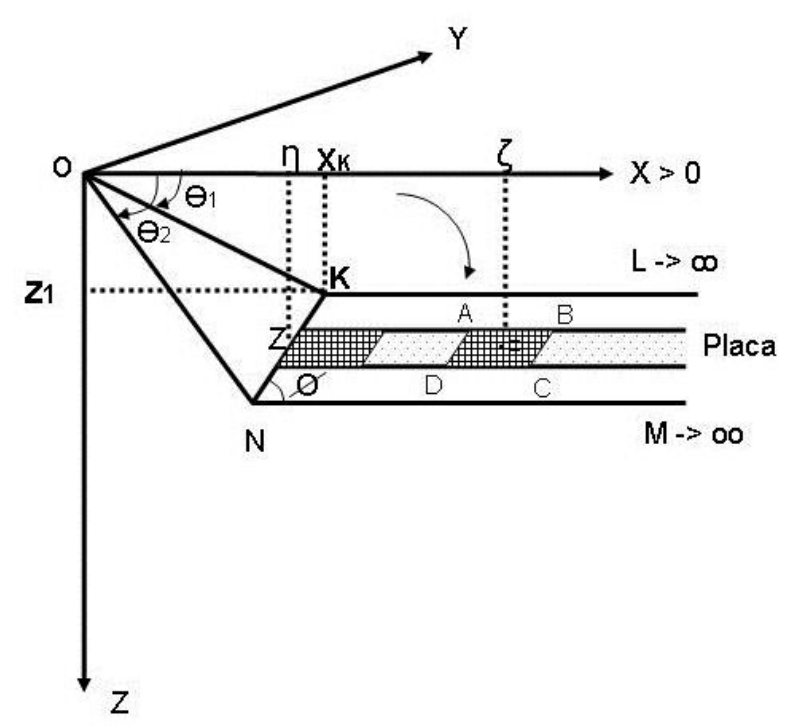

Figura C1. Prisma bidimensional KLMN que se extiende al infinito en el eje $x$ positivo.

Sea

$$
d \vec{m}=\vec{M} d v=\vec{M}_{j} \vec{e}_{j} d v, j=x, y, z
$$

donde $d \vec{m}$ es el momento dipolar magnético, $\vec{e}_{j}$ denota los vectores cartesianos y $\vec{M}$ es la magnetización.

El potencial magnético en el origen debido a una varilla en el punto $P(x, y, z)$ está dado por: 


$$
d V^{m}(O, P)=-d \vec{m}(P) \cdot \vec{\nabla}\left(\frac{1}{r_{O P}}\right)=\frac{d \vec{m}(P) \cdot d \vec{r}_{O P}}{r_{O P}^{3}}
$$

donde $r_{O P}=\left|\vec{r}_{O P}\right|, \quad \vec{r}_{O P}=\vec{x}_{i} \vec{e}_{i}, \quad r_{O P}=\left(x^{2}+y^{2}+z^{2}\right)^{1 / 2}$

$$
d V_{v a r}^{m}(O, P)=\frac{M_{x} x+M_{y} y+M_{z} z}{\left(x^{2}+y^{2}+z^{2}\right)^{3 / 2}} d v(P)
$$

siendo $d v(P)=d x d y d z$

El potencial de la varilla infinita de sección transversal $A B C D$ está dado por:

$$
V_{v a r}^{m}(O, P)=\int_{y=-\infty}^{y=+\infty} d V^{m}(O, P)=d x d y \int_{-\infty}^{+\infty} \frac{M_{x} x+M_{y} y+M_{z} z}{\left(x^{2}+y^{2}+z^{2}\right)^{3 / 2}} d y
$$

Definiendo $C_{x z}=M_{x} x+M_{z} z$ y $a^{2}=x^{2}+z^{2}$, la integral de la ecuación C4 se puede escribir como:

$$
\int_{-\infty}^{+\infty} \frac{C_{x z}+M_{y} y}{\left(a^{2}+y^{2}\right)^{3 / 2}} d y=C_{x z} \int_{-\infty}^{+\infty} \frac{1}{\left(a^{2}+y^{2}\right)^{3 / 2}} d y+M_{y} \int_{-\infty}^{+\infty} \frac{y}{\left(a^{2}+y^{2}\right)^{3 / 2}} d y
$$

Es importante señalar que por la hipótesis de modelo $2 \mathrm{D}, \vec{M}=M_{j}(x, z) \vec{e}_{j}$ no depende de la variable $y$. Por lo tanto $M_{y}$ no depende de $y$. Si bien la contribución de $M_{y}$ es nula, no implica que $M_{y}$ sea cero.

A continuación resolveremos las dos integrales de la ecuación (C5). La primera integral del segundo miembro puede resolverse como:

$$
\begin{gathered}
\int_{-\infty}^{+\infty} \frac{1}{\left(a^{2}+y^{2}\right)^{3 / 2}} d y=\frac{y}{a^{2}\left(a^{2}+y^{2}\right)^{1 / 2}} \|_{-\infty}^{+\infty} \\
=\lim _{L \rightarrow \infty}\left(\frac{L}{a^{2}\left(a^{2}+L^{2}\right)^{1 / 2}}\right) \|_{-L}^{+L}=\lim _{L \rightarrow \infty}\left(\frac{L}{a^{2}\left(a^{2}+L^{2}\right)^{1 / 2}}-\frac{(-L)}{a^{2}\left(a^{2}+L^{2}\right)^{1 / 2}}\right)=\frac{2}{a^{2}}
\end{gathered}
$$

con $L>0$.

Para resolver la segunda integral, hacemos la sustitución $u^{2}=a^{2}+y^{2}, d u=2 y d y$ 


$$
\int_{-\infty}^{+\infty} \frac{y}{\left(x^{2}+y^{2}+z^{2}\right)^{3 / 2}} d y=\frac{1}{2} \int_{-\infty}^{+\infty} u^{-3 / 2} d u=-\frac{1}{\left(a^{2}+y^{2}\right)^{\frac{1}{2}}} \|_{-\infty}^{+\infty}=0
$$

Por lo tanto el potencial magnético de la varilla resulta en la ecuación (C8):

$$
V_{v a r}^{m}(O, P)=V_{v a r}(O ; x, z)=2 d x d z \frac{M_{x} x+M_{z} z}{x^{2}+z^{2}}
$$

Este resultado es independiente de $y$.

A partir de $V_{\text {var }}^{m}(O, P)$ podemos calcular las componentes horizontal y vertical del campo asociado con esta varilla.

$$
\begin{gathered}
H_{x}^{v a r}(O ; x, z)=-\frac{\partial V_{v a r}}{\partial x} \\
H_{z}^{v a r}(O ; x, z)=-\frac{\partial V_{v a r}}{\partial z} \\
H_{x}^{v a r}(O)=2 d x d z \frac{M_{x}\left(x^{2}-z^{2}\right)+M_{z} 2 x z}{\left(x^{2}+z^{2}\right)^{2}} \\
H_{z}^{v a r}(O)=2 d x d z \frac{M_{x} 2 x z+M_{z}\left(z^{2}-x^{2}\right)}{\left(x^{2}+z^{2}\right)^{2}}
\end{gathered}
$$

La fuerza magnética para la placa sombreada en la figura $\mathrm{C} 1$ se puede obtener integrando las componentes $H_{x}^{\text {var }}(O)$ y $H_{z}^{\text {var }}(O)$ respecto de $x$, desde $x$ hasta $\infty$. Tomando como hipótesis que la magnetización es uniforme y $M_{x}$ y $M_{z}$ son independientes de $x$ y $z$ calculamos:

$$
\begin{aligned}
H_{x}^{\text {placa }}(O)=2 d z \int_{x(z)}^{+\infty} \frac{M_{x}\left(x^{2}-z^{2}\right)+M_{z} 2 x z}{\left(x^{2}+z^{2}\right)^{2}} d x \\
\quad=2 M_{x} d z \int_{x(z)}^{+\infty} \frac{x^{2}-z^{2}}{\left(x^{2}+z^{2}\right)^{2}} d x+4 M_{z} z d z \int_{x(z)}^{+\infty} \frac{x}{\left(x^{2}+z^{2}\right)^{2}} d x
\end{aligned}
$$

La componente horizontal de la fuerza magnética para la placa queda expresada como: 


$$
H_{x}^{\text {placa }}(O)=2 d z \frac{M_{x} x+M_{z} z}{x^{2}+z^{2}}
$$

Análogamente

$$
\begin{aligned}
H_{z}^{\text {placa }}(O)=2 d z & \int_{x(z)}^{+\infty} \frac{M_{x} 2 x z-M_{z}\left(x^{2}-z^{2}\right)}{\left(x^{2}+z^{2}\right)^{2}} d x \\
& =4 M_{x} z d z \int_{x(z)}^{+\infty} \frac{x}{\left(x^{2}+z^{2}\right)^{2}} d x-2 M_{z} d z \int_{x(z)}^{+\infty} \frac{x^{2}-z^{2}}{\left(x^{2}+z^{2}\right)^{2}} d x
\end{aligned}
$$

Las integrales del miembro derecho de la ecuación A13 son las mismas que las de la ecuación (C15). Luego

$$
H_{z}^{\text {placa }}(O)=2 d z \frac{M_{x} z-M_{z} x}{x^{2}+z^{2}}
$$

Finalmente, para obtener la fuerza magnética ejercida por el prisma $K L M N$, debemos integrar las expresiones de las placas respecto de $z$, teniendo en cuenta que a lo largo del lado $K N$ las variables $x, z$ están relacionadas linealmente.

$$
\begin{gathered}
H_{x}^{\text {prisma }}(O)=2 \int_{z 1}^{z 2} \frac{M_{x} x+M_{z} z}{x^{2}+z^{2}} d z \\
H_{x}^{\text {prisma }}(O)=2 \operatorname{sen} \emptyset\left[M _ { x } \left(-\cos \emptyset \ln \left(\frac{r_{2}}{r_{1}}\right)\right.\right. \\
\left.\left.+\operatorname{sen} \emptyset\left(\theta_{2}-\theta_{1}\right)\right)+M_{z}\left(\operatorname{sen} \emptyset \ln \left(\frac{r_{2}}{r_{1}}\right)+\cos \emptyset\left(\theta_{2}-\theta_{1}\right)\right)\right] \\
H_{z}^{\text {prisma }}(O)=2 \int_{z 1}^{z 2} \frac{M_{x} z-M_{z} x}{x^{2}+z^{2}} d z \\
H_{z}^{\text {prisma }}(O)=2 \operatorname{sen} \emptyset\left[M_{x} \operatorname{sen} \emptyset \ln \left(\frac{r_{2}}{r_{1}}\right)\right. \\
\left.+\cos \emptyset\left(\theta_{2}-\theta_{1}\right)-M_{z}\left(-\cos \emptyset \ln \left(\frac{r_{2}}{r_{1}}\right)+\operatorname{sen} \emptyset\left(\theta_{2}-\theta_{1}\right)\right)\right]
\end{gathered}
$$

La anomalía magnética causada por un cuerpo de sección poligonal KNPQRK puede ser evaluada por medio de anomalías producidas por prismas como $K L M N$ con su signo 
correcto como se indica en la figura C2. Si se avanza en sentido horario a lo largo de los vértices del polígono $K N P Q R$, para los segmentos tales como $K N$ (para los cuales $\theta$ es creciente), la anomalía causada por el prisma correspondiente $K L M N$ debe ser tomada con signo negativo.

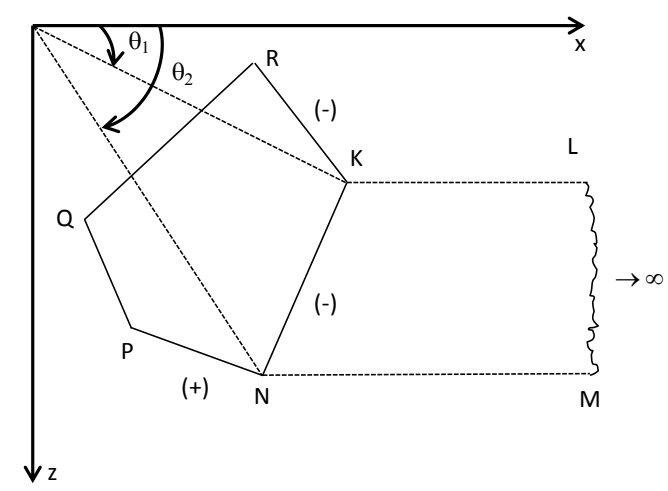

Figura C2. Construcción geométrica usada para calcular la anomalía causada por un cuerpo de sección poligonal KNPQRK.

Por lo tanto, las ecuaciones C18 y C20 para el cálculo de la anomalía magnética pueden reescribirse como:

$$
\begin{aligned}
& H_{x}^{\text {prisma }}(O)=2\left(M_{x} Q-M_{z} P\right) \\
& H_{z}^{\text {prisma }}(O)=2\left(M_{x} P+M_{z} Q\right)
\end{aligned}
$$

donde

$$
\begin{gathered}
P=\operatorname{sen}^{2} \emptyset\left(\theta_{2}-\theta_{1}\right)+\cos \emptyset \operatorname{sen} \emptyset \ln \left(\frac{r_{2}}{r_{1}}\right) \\
Q=\cos \emptyset \operatorname{sen} \emptyset\left(\theta_{2}-\theta_{1}\right)-\operatorname{sen}^{2} \emptyset \ln \left(\frac{r_{2}}{r_{1}}\right) \\
x_{12}=x_{1}-x_{2}, \quad z_{21}=z_{2-} z_{1}, \quad r_{1}=\sqrt{x_{1}^{2}+z_{1}^{2}}, \quad r_{2}=\sqrt{x_{2}^{2}+z_{2}^{2}} \\
\operatorname{sen} \emptyset=\frac{z_{2}-z_{1}}{\left(\left(x_{1}-x_{2}\right)^{2}+\left(z_{2}-z_{1}\right)^{1 / 2}\right.}=\frac{z_{21}}{\left(x_{12}^{2}+z_{21}^{2}\right)^{1 / 2}} \\
\cos \emptyset=\frac{x_{1}-x_{2}}{\left(\left(x_{1}-x_{2}\right)^{2}+\left(z_{2}-z_{1}\right)^{1 / 2}\right.}=\frac{x_{12}}{\left(x_{12}^{2}+z_{21}^{2}\right)^{1 / 2}}
\end{gathered}
$$




$$
\begin{aligned}
& P=\left(\frac{z_{21}^{2}}{z_{21}^{2}+x_{12}^{2}}\left(\theta_{2}-\theta_{1}\right)+\frac{z_{21} x_{12}}{z_{21}^{2}+x_{12}^{2}}\right) \ln \left(\frac{r_{2}}{r_{1}}\right) \\
& Q=\left(\frac{z_{21} x_{12}}{z_{21}^{2}+x_{12}^{2}}\left(\theta_{2}-\theta_{1}\right)-\frac{z_{21}^{2}}{z_{21}^{2}+x_{12}^{2}}\right) \ln \left(\frac{r_{2}}{r_{1}}\right)
\end{aligned}
$$

Estas expresiones son válidas para cada segmento del polígono si se recorre en sentido de las agujas del reloj. Los índices 1 y 2 indican vértices sucesivos en cada caso. Un perfil magnético se calcula moviendo el origen sucesivamente a varios puntos a lo largo del eje $x$.

Para evaluar $M_{x}$ y $M_{z}$ se adopta la siguiente convención (figura C3). Sea A la inclinación de $\vec{M}$, positiva debajo del horizonte y B el ángulo entre su proyección horizontal medida en la dirección de las agujas del reloj y el norte geográfico.

Sea $\mathrm{C}$ el ángulo entre la dirección positiva de $x$ y el norte geográfico medido en sentido horario.

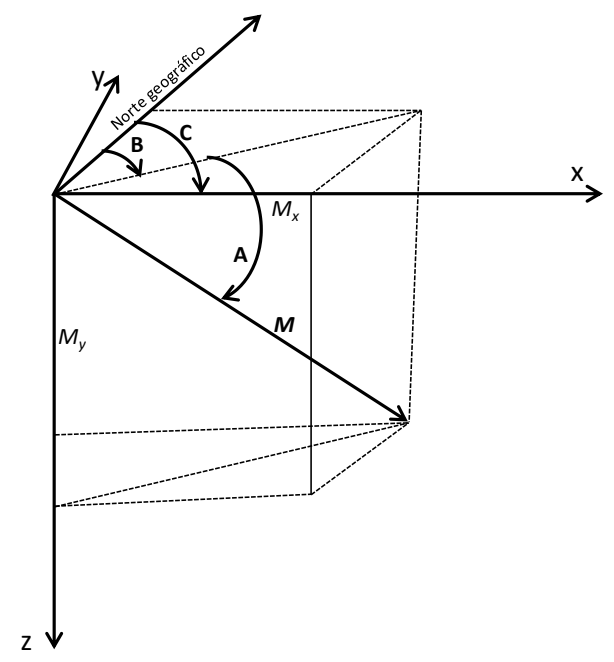

Figura C3. Elementos que definen a las componentes del vector de magnetización.

$$
\begin{gathered}
M_{x}=M \cos A \cos (C-B) \\
M_{z}=M \operatorname{sen} A
\end{gathered}
$$

Si la magnetización es por inducción, entonces $\vec{M}=\kappa \vec{F}, \mathrm{y} A=\mathrm{I}, \mathrm{B}=\mathrm{D}$, donde $I$ y $D$ son las direcciones de la inclinación y declinación magnética respectivamente del campo terrestre. 
Si la anomalía total $T$ es pequeña con respecto al campo total $F$, entonces $T$ es la suma de las proyecciones de $H_{x}$ y $H_{z}$ en la dirección de $F$, es decir:

$$
T=H_{z} \operatorname{sen} \mathrm{I}+H_{x} \cos \mathrm{I}+\cos (\mathrm{C}-\mathrm{D})
$$




\section{REFERENCIAS}

Aisengart, A., 2015, Qualitative and Quantitative Magnetization Vector Inversion applied to the Pirapora Anomaly, 14th International Congress of the Brazilian Geophysical Society, Rio de Janeiro, Brazil, August 3-6, 2015.

Atchuta Rao, D., Ram Babu, H.V. \& Sanker Narayan, P.V., 1981, Interpretation of magnetic anomalies due to dikes: the complex gradient method. Geophysics, 46, p. 1572-1578.

Baranov, V., 1957. A new method for interpretation of aeromagnetic maps; pseudogravimetric anomalies, Geophysics, 22, 359-383.

Barbosa V., Silva J. \& Mediros, W., 1999, Stability analysis improvement of structural index estimation in Euler deconvolution, Geophysics, 64, p. 48-60.

Barbosa V., Silva J. \& Mediros, W., 2000, Making Euler deconvolution applicable to small ground magnetic surveys, Journal of Applied Geophysics, 43, p. 55-68.

Bermúdez, A., Delpino, F. D., Zencich, S. \& Bolatti, N., 2000, Evolución volcanológica y petrológica del sector sur del campo volcánico Auca Mahuida, Provincia del Neuquén, Argentina, 2000. IX Congreso Geológico Chileno, Actas Vol. 2, Sesión temática º 6, p. $1-5$.

Bermúdez, A. \& Delpino, D., 1998, Estudio de testigos corona de rocas ígneas intrusivas reservorios de hidrocarburos y de las secuencias del Volcán Auca Mahuida. Repsol YPF. Reporte sin publicar.

Bermúdez, A. \& Delpino, D., 2002, Estudio geoquímico de cutting de cuerpos intrusivos de pozos en el área del yacimiento Volcán Auca Mahuida. Repsol YPF. Reporte sin publicar.

Bhattacharyya, B. K., 1966, Continuous spectrum of the total-magnetic-field anomaly due to a rectangular prismatic body, Geophysics 31, p. 97-121.

Blakely, R. J., 1981, A program for rapidily computing the magnetic anomaly over digital topography. Open-File Report 81-298. 
Blakely, R. J., 1995, Potential Theory in Gravity and Magnetic Applications, Cambridge University Press, New York, 441 pp.

Blanco Montenegro, I., 1997, Análisis e interpretación de las anomalías magnéticas de tres calderas volcánicas: Decepción (Shetland del Sur, Antártica), Furnas (San Miguel, Azores) y Las Cañadas del Teide (Tenerife, Canarias). Tesis Doctoral. Facultad de Ciencias Físicas, Universidad Complutense de Madrid, 215 pp.

Blanco Montenegro, I., Miquel Torta, J., García, A. \& Araña, V., 2003, Analysis and modelling of the aeromagnetic anomalies of Gran Canaria (Canaria Island), Earth and Planetary Science Letters 206, p. 601-616, doi:10.1016/S0012-821X(02)01129-9.

Blanco Montenegro, I., De Ritis R. \& Chiappini M., 2006, Imaging and modelling the subsurface structure of volcanic calderas with high-resolution aeromagnetic data at Vulcano (Aeolian islands, Italy), B. Volcanol., 69 (6), p. 643-659, doi:10.1007/s00445-581 006-0100-7.

Brisson I. E. \& Veiga R. D., 1999, Gira de campo. Cuenca Neuquina. YPF, Informe interno.

Butler, R.F., 1992, Paleomagnetism: Magnetic domains to geologic terranes, Blackwell Scientific Publications Boston, 319 pp. http://lewis.up.edu/chp/butler/books/main.htm.

Carson Aerogravity, 2001, Volcán Auca Mahuida and Señal Cerro Bayo exploration lots. Airbone gravity and magnetic survey for Repsol YPF. Data processing report.

Comeron, R., González, J. M. \& Schiuma, M., 2002, Los reservorios de las rocas ígneas intrusivas. En: Schiuma, M., Hinterwimmer, G. \& Vergani, G. eds. Rocas reservorio de las cuencas productivas de Argentina. V Congreso de Exploración y desarrollo de Hidrocarburos, Mar del Plata, p. 559-582.

Constable, S. C., Parker, R. L. \& Constable, C. G., 1987, Occam's inversion: A practical algorithm for generating smooth models from electromagnetic sounding data, Geophysics 52, p. 289-300.

Cox, A., Magnetostratigraphic time scale, In: A Geologic Time Scale, ed. W. B. Harland et al., Cambridge University Press, Cambridge, England, p. 63-84, 1982.

Cristallini, E. O., Bottesi, G., Gavarrino, A., Rodríguez, L., Tomezzoli, R. \& Comeron, R., 2006, Synrift geometry of the Neuquén Basin in the northeastern Neuquén Province, Argentina. In: Kay, S. M. \& Ramos, V. A. eds. Evolution of the Andean margin: a tectonic 
and magmatic view from the Andes to the Neuquén Basin $\left(35^{\circ}-39^{\circ}\right.$ latitude). Geological Society of America, Special Paper, 407, p. 147-161.

De Ritis, R., 2004, Analisi integrata del campo di anomalia magnetica e delle caratteristiche vulcanologiche-strutturali di vulcano(isole eolie), Tesis Doctoral, Dipartimento di Fisica, Facoltà di Scienze Matematiche Fisiche e Naturali, Università degli Studi di Bologna.

De Ritis, R., Ventura, G., Chiappini, M., Carluccio, R. \& Von Frese, R. 2010, Regional magnetic and gravity anomaly correlations of the Southern Tyrrhenian Sea. Phys. Earth Planet. Inter., doi:10.1016/j.pepi.2010.04.003.

Delgado de la Puente, F. J., 2010, Estructura interna de la caldera La Pacana (II Región) mediante gravimetría. Tesis para optar al grado de magister en ciencias, mención geofísica. Facultad de Ciencias Físicas y Matemáticas, Departamento de Geofísica, Departamento de Geología, Universidad de Chile, pp. 182.

Delpino, D., 2002, Relevamiento geológico de superficie del campo volcánico Auca Mahuida, Provincia del Neuquén, Mapa interno Repsol-YPF.

Fairhead, J. D., 2016, Advances in Gravity and Magnetic Processing and Interpretation, EAGE Publications bv, pp. 352.

Ferpozzi, F., Peñalva, G. \& Vargas D., 2007, Bloque Cuenca Neuquina 1968; Levantamiento aeromagnético analógico: digitalización, reprocesamiento y edición. Servicio Geológico Minero Argentino, Informe sin publicar.

Finn, C., Sisson, T.W. \& Deszcz-Pan, M., 2001, Aerogeophysical measurements of collapsephrone hydrothermally altered zones at Mount Rainer volcano, Nature, 409, p. 600603.

Geosoft, 2008, Montaj grav-mag interpretation, Processing, Analysis and Visualization System for 3D Inversion of Potential Field Data for Oasis Montaj v6.3, Tutorial and User Guide.

Gunn, P. J., 1997, Quantitative methods for interpreting aeromagnetic data: a subjective review. AGSO Journal of Australian Geology \& Geophysics, 17 (2), p. 105-113.

Heiskanen, W. A. \& Morits, H., 1967, Physical Geodesy, Freeman W. H. \& Co. Ltd, San Francisco, $364 \mathrm{pp}$.

Henderson, R. G., 1970, On the validity of the use of the upward continuation integral for total 
magnetic intensity data, Geophysics 35, p. 916-19.

Hinze, W. J., Von Frese, R. R. B. \& Saad, A. H., 2013. Gravity and Magnetic Exploration, pp. 525, Cambridge University Press.

Holmberg, E. 1964, Descripción geológica de la Hoja 33d, Auca Mahuida (Provincia del Neuquén). Dirección Nacional de Geología y Minería, Buenos Aires, Boletín, 94, p. 188.

Hsu, S., 2002, Imaging magnetic sources using Euler's equation. Geophysical Prospecting, 50, p. $15-25$.

Kane, M. F., 1962, A Comprehensive System of Terrain Using a Digital Computer, Geophysics 27 (4), p. 455-462.

Kay, S. M., Gorring, M. \& Ramos, V. A., 2004, Magmatic sources, setting and causes of Eocene to Recent Patagonian plateau magmatism $\left(36^{\circ} \mathrm{S}\right.$ to $52^{\circ} \mathrm{S}$ latitude), Revista de la Asociación Geológica Argentina, 59 (4), p. 556-568.

Kay, S. M., Burns, M., \& Copeland, P., 2006, Upper Cretaceous to Holocene magmatism and evidence for transient Miocene shallowing of the Andean subduction zone under the northern Neuquén Basin. In: Kay S. M. \& Ramos, V. A. eds. Evolution of an Andean margin: a tectonic and magmatic view from the Andes to the Neuquén Basin $\left(35^{\circ}-\right.$ $39^{\circ}$ S). Geological Society of America, Special Paper, 407, p. 19-60.

Kirschvink, J. L., 1980, The least-squares line and plane and the analysis of paleomagnetic data, Geophysical, J. R. Astron. Soc., 62, p. 699-718.

Lince Klinger, F., León, M., Martínez, P., Weidmann, C., Anci, S., Álvarez, O., 2014, Modelo geofísico con datos gravimétricos y aeromagnéticos en el borde noreste del macizo norpatagónico, Río Negro, Argentina, Geoacta 39(1): 51-61 (2014), Asociación Argentina de Geofísicos y Geodestas, ISSN 1852-7744.

Lince Klinger, F., Orts D., Gimenez, M., Folguera, A. \& Martinez, P., 2015, Multiple geophysical methods used to examine neotectonic structures in the western foothills of the Sierra de El Maitén (Argentina), North Patagonian Andes, Near Surface Geophysics, 2016, doi:10.3997/1873-2016003.

Llambías, E. J., Bertotto G. W., Risso C. \& Hernando I., 2010, El volcanismo Cuarternario en el retroarco de Payenia: una revision. Revista de la Asociación Geológica Argentina, 67 (2), p. 278-300. 
Longo, L. M., 2000, Prospección de hidrocarburos en la Cuenca Neuquina aplicando métodos sísmicos de reflexión. Tesis de grado para optar al título de Geofísica. Facultad de Ciencias Astronómicas y Geofísicas, Universidad Nacional de La Plata.

Longo, L. M., Ravazzoli, C. L. \& Chiappini, M., 2008, Proyecto de interpretación de datos aerogravimétricos y magnéticos en el Volcán Auca Mahuida. Cuarto encuentro ciéntifico del ICES (E-ICES4), Malargüe, Mendoza, Argentina.

Longo, L. M., De Ritis, R., Ventura, G. \& Chiappini, M., 2015, Analysis of the Aeromagnetic Anomalies of the Auca Mahuida Volcano, Patagonia, Argentina, Pure and Applied Geophysics, 172 (8), p. 2083-2332. doi :10.1007/s00024-015-1161-3.

López Loera, H., Urrutia-Fucugauchi, J. \& Alva-Valdivia, L., 2011, Estudio aeromagnético del complejo volcánico de Colima, occidente de México - implicaciones tectónicas y estructurales, Revista Mexicana de Ciencias Geológicas, 28 (3), p. 349-370.

Lowrie, W., Fundamentals of Geophysics (Cambridge University Press, New York, 2007, 393 pp.) www.cambridge.org/9780521859028.

Mandea M. \& Macmillan S., 2000, International Geomagnetic Reference Field - The Eighth Generation. Earth, Planets and Space 52, 1119-1124.

MacLeod, I.N, Jones, K. \& Fan Dai, T., 1993, 3-D analytic signal in the interpretation of total magnetic field data at low magnetic latitudes. Explor. Geophys., 24, p. 679-688.

MacLeod, I. N. \& Ellis, R. G., 2013, Magnetic Vector Inversion, a simple approach to challenge of varying direction of rock magnetization. $23 \mathrm{rd}$ International Geophysical conference and exhibition, 11-14 August 2013. ASEG-PESA Melbourne, Australia.

Mankinen, E. A. \& Dalrymple G. B, 1979, Revised geomagnetic polarity time scale for the interval 0-5 m.y. B.P., Journal of Geophysical research, Solid Earth, 84, B2 10, p. 615626, doi: 10.1029/JB084iB02p00615.

Masturyono, McCaffrey, R., Wark, D. A., Roecker S. W., Fauzi, Ibrahim, G. \& Sukhyar, 2001, Distribution of magma beneath the Toba caldera complex, north Sumatra, Indonesia, constrained by three-dimensional $\mathrm{P}$ waves velocities, seismicity, and gravity data, American Geophysical Union (AGU) and Geochemical Society, vol. 2, 2000GC000096, ISSN: 1525-2027.

Maus, S. \& Dimri, V., 1996, Depth estimation from the scaling power spectrum of potential 
fields? Geophys. J. Int., 124, p. 113-120.

McElhinny, M.W. 1973, Palaeomagnetism and Plate Tectonics (Cambridge University Press, London, 358 pp.).

Mosquera, A. \& Ramos, V.A., 2006, Intraplate deformation in the Neuquén Basin. In: Kay S. M. \& Ramos, V. A. eds. Evolution of an Andean margin: a tectonic and magmatic view from the Andes to the Neuquén Basin $\left(35^{\circ}-39^{\circ} \mathrm{S}\right.$ latitude). Geological Society of America, Special Paper, 407, p. 97-124.

Mushayandebvu, M., Van Driel, P., Reid, A. \& Fairhead, J., 2001, Magnetic sourece parameters of two-dimensional structures using extended Euler deconvolution. Geophysics 66, p. 814-823.

Nabighian, N. M., 1972, The analytic signal of two-dimensional magnetic bodies with polygonal cross section: its properties and used for automated anomaly interpretation. Geophysics 37, p. 507-517.

Nabighian, N. M., 1984, Toward a three-dimensional automatic interpretation of potential field data via generalized Hilbert transforms: fundamental relations, Geophysics 49, p. 780-786.

Nagy, D., 1966, The Gravitational Attraction of a Right Rectangular Prism, Geophysics 31 (2), p. 362-371.

Naidu, P. \& Mathew, M., 1998, Analysis of geophysical potencial fields: A digital signal processing approach, Elsevier.

Nettleton, L. L., 1939, Determination of density for reduction of gravimeter observations, Paper read at Annual Meeting, Oklahoma City, Oklahoma, March 21, 1939.

Nettleton, L. L., 1940, Geophysical Prospecting for Oil, New York ; London : McGraw-Hill; 1St Edition edition.

Ortega Estupiñán, A. M., 2014, Modelo de fuentes de anomalías gravimétricas regional y locales del volcán Galeras, asociadas a su estado de actividad entre junio 2008 - abril de 2009. Magister en Ciencias - Geofísica, Facultad de Ciencias, Departamento de Geociencias, Universidad Nacional de Colombia, 92 pp.

Paine, J., De Ritis, R., Ventura, G., Longo, L. M., Ravat, D., Speranza, F. \& Chiappini, M., 2015, 
Inverse modeling of the reversely magnetized, shallow plumbing system hosting oil reservoirs of the Auca Mahuida volcano (Payeina retroarc, Neuquén Basin, Argentina), Geophysical Journal International, p 852-867, doi: 10.1093/gji/ggv487.

Pallares C., Quidelleur, X., Gillot, P. Y. \& Kluska, J. M., 2016, The temporal evolution of back-arc magmas from the Auca Mahuida shield volcano (Payenia Volcanic Province, Argentina), Journal of Volcanology and Geothermal Research, 323, p. 19-37.

Pángaro, F., Villar, H., Vottero, A., Bojarski, G., Rodríguez Arias, L., 2004, Eventos volcánicos y sistemas petroleros: El caso del Volcán Auca Mahuida, Cuenca Neuquina, Argentina. IX Congreso Latinoamericano de Geoquímica orgánica, México, 23-27.

Pángaro, F., Martínez, R., Sattler, F. \& Bettini, F., 2011, El Bajo de Añelo, Relatorio del XVIII Congreso Geológico Argentina, Neuquén.

Parker, R. L., 1972, The rapid calculation of potential anomalies, Geophys. J. Roy.Astr. Soc. 31: 447-455.

Profeta, M., 2000, Informe sobre la recopilación, procesamiento e interpretación de los gravimétricos terrestres correspondientes a la Cuenca Neuquina y la Patagonia (República Argentina). Informe interno YPF S. A.

Ramos, V. A. \& Kay, S. M., 2006, Overview over the tectonic evolution of the southern Central Andes of Mendoza and Neuquén (35॰-39॰ S latitude). In: Kay, S. M. \& Ramos, V. A. eds. Evolution of an Andean margin: a tectonic and magmatic view from the Andes to the Neuquén Basin (35॰-39॰ S latitude). Geological Society of America, Special Paper, 407, p. 1-18.

Ramos, V. A. \& Folguera, A., 2010, Payenia volcanic province in the Southern Andes: an appraisal of an exceptional Quaternary tectonic setting. Journal of Volcanology and Geothermal Research, 201, p. 53-64. doi:10.1016/j.jvolgeores.2010.09.008.

Reid, A., Allsop, J. Granser, H., Millet, A. \& Somerton, I., 1990, Magnetic interpretation in three dimensions using Euler deconvolution. Geophysics, 55, p. 80-91.

Roest, W. R., Verhoeff, J. \& Pilkington, M., 1992, Magnetic interpretation using the 3-D analytic signal. Geophysics 57, p. 116-125.

Rossello, E. A., Cobbold, P. R., Diraison, M. \& Arnaud N., 2002, Auca Mahuida (Neuquén basin, Argentina): A Quaternary shield volcano on a hydrocarbon-producing substrate, in $6^{\text {th }}$ International Symposium on Andean Geodynamics (ISAG 2002). Extended Abstracts, 
Barcelona, Univ. De Barcelona: Instituto Geológico y Minero de España, p. 549-552.

Salem, A. \& Ali, M. Y., 2016, Mapping basement structures in the northwestern offshore of Abu Dhabi from high-resolution aeromagnetic data, Geophysical Prospecting, 64, 726-740, doi: 10.1111/1365-2478.12266.

Sánchez Jiménez, N., 2003, Estructura gravimétrica y magnética de la corteza del suroeste peninsular (zona Surportugueza y zona de Ossa-Morena). Tesis Doctoral. Facultad de C.C. Geológicas, Departamento de Geodinámica, Universidad Complutense de Madrid, $243 \mathrm{pp}$.

Schlumberger, 1987, Evaluación de formaciones en la Argentina. Trabajo técnico.

Schlumberger, 1995, Seminario de interpretación de perfiles. Principios, aplicaciones de interpretación de registros, Buenos Aires.

Shirman, B., Rybakov, M., Beyth, M., Mushkin, A. \& Ginat, H., 2015, Deep structure of the Mount Amram igneous complex, interpretation of magnetic and gravity data, Geophysical Journal International, 200, p. 1362-1373, doi: 10.1093/gji/ggu461.

Sigismondi, M. E., 2011, El estiramiento cortical de la Cuenca Neuquina: modelo de cizalla simple, Relatorio del XVIII Congreso Geológico Argentina, Neuquén.

Sigismondi, M. E., 2012, Estudio de la deformación litosférica de la Cuenca Neuquina: estructura termal, datos de gravedad y sísmica de reflexión. Tesis Doctoral. Facultad de Ciencias Exactas y Naturales, Universidad de Buenos Aires, p. 367.

Spector, A. \& Grant, F. S., 1970, Statistical models for interpreting aeromagnetic data, Geophysics, 35, p. 293-302.

Talwani, M. \& Heirtzler, J. R., 1964, Computation of magnetic anomalies caused by twodimensional bodies of arbitrary shape, In: G.A. Park (Ed.), Computers in Mineral Industries, Part 1, edn. Stanford Univ. Publ., Geological Sciences 9, p. 464-480.

Tauxe, L., 2005, Lectures in Paleomagnetism. http://earthref.org/MAGIC/books/Tauxe/2005/.

Thompson, D., 1982, EULDPH: A new technique for making computer-assisted depth estimates form magnetic data. Geophysics, 47, p. 31-37.

Tikohonov, A. N., Goncharsky, A. V., Stepanov, V. V. \& Yagola A. G., 1995, Numerical Methods for the solution of ill-posed problems, Springer-Science-Business Media Dordrecht, 
doi: 10.1007/978-94-015-8480-7.

Ventura G., De Ritis R., Longo M. \& Chiappini M., 2012, Terrain characterization and structural control of the Auca Mahuida volcanism (Neuquén Basin, Argentina), International Journal of Geographical Information Science. International Journal of Geographical Information Science iFirst, 2012, p. 1-12.

Vottero, A. 2002, Yacimiento Volcán Auca Mahuida: desarrollo y protección ambiental. V Congreso de Exploración y Desarrollo de Hidrocarburos, Mar del Plata, Argentina.

Vottero, A., Rodríguez, L. \& Vela, R., 2005. Trampas de hidrocarburos en el centro este de la Cuenca Neuquina. VI Congreso de Exploración y Desarrollo de Hidrocarburos- Las trampas de hidrocarburos en las cuencas productivas de Argentina, 189-208.

Wohletz, K. \& Heiken, G., Volcanology and Geothermal Energy, Berkeley: University of California Press, 1992, http://ark.cdlib.org/ark:/13030/ft6v19p151/.

Won, I. J. \& Bevis, M., 1987, Computing the gravitational and magnetic anomalies due to a polygon: Algorithms and Fortran subroutines, Geophysics 52, p. 232-238.

Yilmaz, 0, 1988, Seismic data processing, Investigations in Geophysics, Vol. 2. Society of Exploration, Tulsa, Oklahoma, U.S.A.

Zapata, T. \& Folguera, A., 2005, Tectonic evolution of the Andean fold and thrust belt of the Southern Neuquén basin, Argentina. In: Veiga, G., Spalletti, L., Howell, J., Schwarz, E. eds. The Neuquén Basin: A Case Study in Sequence Stratigraphy and Basin Dynamics. Geological Society of London, Special Pubblication, 252, p. 37-56.

Zijderveld, J. D. A., 1967, A.C. demagnetization of rocks: Analysis of results, In: Methods in Palaeomagnetism, Collinson, D.W., K.M. Creer, and S.K. Runcorn (Eds), Elsevier, New York, p. 254-286. 